\title{
TETRACHLOROBIPHENYL METABOLISM, TOXICITY, AND REGULATION OF CYTOCHROME P450 EXPRESSION IN A MARINE TELEOST FISH
}

by

\author{
Renee Devorah White \\ B.A. Molecular Biology and Biochemistry, \\ Wesleyan University, Middletown, CT, 1988 \\ SUBMITTED IN PARTIAL FULFILLMENT OF THE \\ REQUIREMENTS FOR THE DEGREE OF \\ DOCTOR OF PHILOSOPHY \\ at the \\ MASSACHUSETTS INSTITUTE OF TECHNOLOGY \\ and the \\ WOODS HOLE OCEANOGRAPHIC INSTITUTION
}

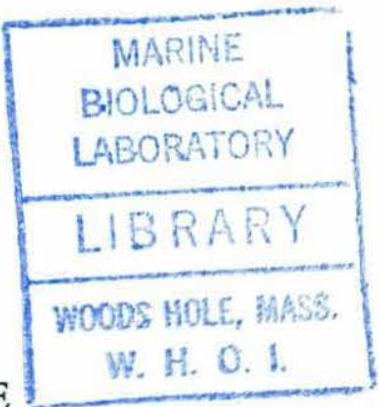

November, 1994

(C) Renee D. White, 1995

The author hereby grants to MIT and WHOI permission to reproduce and distribute copies of this thesis document in whole or in part

Signature of Author

Joint Program in Oceanography,

Massachusetts Institute of Technology/Woods Hole Oceanographic Institution

Certified by

Accepted by

Chair, Joint Committee for Biological Oceanography,

Massachusetts Institute of Technology/Woods Hole Oceanographic Institution 


\title{
TETRACHLOROBIPHENYL METABOLISM, TOXICITY, AND REGULATION OF CYTOCHROME P450 EXPRESSION
}

\section{IN A MARINE TELEOST FISH}

by

\author{
Renee Devorah White
}

\begin{abstract}
submitted in partial fulfillment of the requirements for the degree of Doctor of Philosophy
\end{abstract}

\begin{abstract}
The effects of 3,3',4,4'-tetrachlorobiphenyl (TCB) were examined in the marine fish scup (Stenotomus chrysops), focusing on the interactions between TCB and the CYP1A1 enzyme system. A low TCB dose $(0.1 \mathrm{mg} / \mathrm{kg})$ elicited strong and sustained induction of hepatic CYP1A1 mRNA, protein content, and catalytic activity. A high TCB dose $(5.0 \mathrm{mg} / \mathrm{kg})$ elicited similar, strong induction of hepatic CYP1A1 mRNA, but not of CYP1A1 protein content or catalytic activity. This post-transcriptional "suppression" at the high TCB dose was specific for CYP1A1, and was not seen with other hepatic enzymes. In vitro studies indicate that hepatic microsomal CYP1A1 is inactivated in the presence of TCB plus cofactor, likely due to the production of reactive oxygen species during TCB occupation of the active site. CYP1A1 inactivation by TCB in vitro may explain the TCBelicited suppression of CYP1A1 protein content in vivo.

Both TCB doses elicited strong CYP1A1 induction in vascular endothelium of all organs, which was sustained for several weeks. Induction in intestinal epithelia was stronger at the high TCB dose, but induction in epithelia of liver, kidney, and gill were stronger at the low TCB dose. Both TCB doses caused proliferation of endoplasmic reticulum in liver, renal tubule necrosis, depletion of hematopoietic tissue in kidney, hyperplasia of gill epithelia, and increased number of melanomacrophage aggregates in spleen. The high dose induced tail fin erosion, affecting both epithelial and calcified bone tissue. Tissue alterations were more severe at the high TCB dose, and repair of lesions occurred by day 16 at the low dose. The high dose caused mortality of many individuals.

The two TCB congeners 3,3',4,4'-TCB and 2,2',5,5'-TCB were each converted to aqueous-soluble metabolites by hepatic microsomes from scup, beluga whale, and pilot whale. Induction response, correlation analysis, and inhibition studies indicate that $3,3^{\prime}, 4,4^{\prime}-\mathrm{TCB}$ is metabolized by scup CYP1A1, and 2,2',5,5'-TCB by the putative scup CYP2B. Correlation analysis and inhibition studies suggest that $3,3^{\prime}, 4,4^{\prime}-\mathrm{TCB}$ is metabolized by cetacean CYP1A. Both cetacean species expressed microsomal proteins that are immunochemically related to mammalian CYP2B forms. Cytochrome P450 systems from both cetacean species are partially characterized here.

Thesis Supervisor: John J. Stegeman, Senior Scientist, WHOI
\end{abstract}




\section{ACKNOWLEDGMENTS}

First, I would like to thank my thesis supervisor, John Stegeman, for his advice in helping to shape my research projects, for reminding me to be critical of my own work, for teaching me to choose my words carefully, and for his positive feedback and excellent editorial skills. I thank my committee member Mark Hahn, for offering valuable insights into the data, and a constant flow of reprints. I thank Judy McDowell, my thesis supervisor during my first two years here and then a committee member, for her support, her positive attitude, and her helpful comments on the chapters. I would also like to thank my other committee members: Steve Tannenbaum (M.I.T.) for traveling to Woods Hole to attend committee meetings, and for offering valuable input into the research as it progressed; David Kupfer (Worcester Foundation for Experimental Biology) for long talks about my results and other topics during NUTMEG meetings; Jay Gooch (Proctor and Gamble), whose early work on TCB/CYP1A1 interactions in John Stegeman's lab provided a starting point for much of my thesis research; and John Farrington (committee member emeritus), for his participation in the proposal defense. I would also like to thank John Teal, who offered his time to chair both the proposal defense and the thesis defense.

I also owe a profound thanks to those people in the laboratory who provided the day-to-day advice that made this work possible. I especially thank Michael Moore and Bruce Woodin, who have been infinitely generous with their time and expertise. Both helped to collect and prepare some of the scup tissue samples used in the thesis research, Michael has answered countless questions about histology, and Bruce has always been there to answer questions about laboratory techniques. Roxanna Smolowitz (M.B.L.) has provided expert advice on histopathology, for which I am very grateful. Several people advised me on mRNA techniques, including Grace Bruning (M.B.L.), Bonnie Woodward, Sibel Karchner, and Margie Oleksiak.

I would like to thank those people with whom I collaborated on laboratory projects. Andy Solow (WHOI, Marine Policy Center) developed a statistical analysis for the data from treated scup. Damian Shea (North Carolina State University) analyzed PCB residues and PCB metabolites in the treated scup samples. Lyle Lockhart (Department of Fisheries and Oceans, Winnipeg) collaborated on a study of P450 in beluga whales, and provided PCB residue analyses of these samples. Jennifer Joy has collaborated with me on an analysis of pilot whale liver biochemistry, and on an experiment of TCB distribution in scup. I also thank Neal Cornell (M.B.L.) and Hillary Morrison, who supplied nucleotide probes used in the research. And I am grateful to those who generously provided tissue samples for use in the thesis research: Mark Melancon (U.S. Fish and Wildlife Service, 
Laurel, MD) donated rat liver microsomes, Greg Early (New England Aquarium) and Michael Moore provided access to pilot whale liver samples, and Lyle Lockhart and Lothar Dahlke (Department of Fisheries and Oceans, Winnipeg) provided beluga whale liver samples.

I would like to acknowledge every member of the Stegeman lab with whom I have worked over the years. These people have made the work more enjoyable, and have provided distractions from work whenever they were needed. I especially acknowledge Margie Oleksiak, Jennifer Joy, Connie Hart, Malin Celander, Rachel Cox, Sibel Karchner, Roxanna Smolowitz, Bonnie Woodward, Michael Moore, Bruce Woodin, Mark Hahn, and John Stegeman.

I also owe thanks to my friends and family, for their support, and for helping me to remember that there is more to life than writing one's thesis. I especially thank my graduate student cohorts Susie McGroddy, Cheri Recchia, Alan Kuo, and Michele Durand, and my family, Myra White, Lawrence White, Dan White, Ken White, Barbara White, Suzanne Sack, Audrey Stahl, and of course Michael Cassell, for his tireless support, and Chunky.

This research was funded in part by the Massachusetts Institute of Technology / Woods Hole Oceanographic Institution Joint Doctoral Program; by NIH grant P30ES02109; by EPA grant R817988; and by grant NA-90-AA-D-SG480 from the Coastal Ocean Program of the National Oceanic and Atmospheric Administration to the Woods Hole Oceanographic Institution Sea Grant Program. 
Abstract 3

$\begin{array}{ll}\text { Acknowledgments } & 5\end{array}$

$\begin{array}{ll}\text { List of Tables } & 10\end{array}$

$\begin{array}{ll}\text { List of Figures } & 11\end{array}$

$\begin{array}{ll}\text { Abbreviations } & 13\end{array}$

$\begin{array}{ll}\text { CHAPTER ONE: Introduction } & 15\end{array}$

Introduction

Cytochrome P450 Structure and Function

Cytochrome P450 Regulation

Cytochrome P450 in Marine Vertebrates

Polychlorinated Biphenyls: Distribution, Toxicity, Metabolism

Interaction Between Polychlorinated Biphenyls and Cytochromes P450

Goals of Dissertation Research

CHAPTER TWO: 3,3',4,4'-Tetrachlorobiphenyl Elicits Induction,

in vivo and in vitro
Abstract
Introduction
Materials and Methods
Results
Discussion

CHAPTER THREE: Temporal Pattern of Induction of Cytochrome P4501A1 in Multiple Organs and Cell Types of the Marine Fish Scup Treated with Low and High Doses of 3,3',4,4'-Tetrachlorobiphenyl
Abstract
Introduction
Materials and Methods 
Results

Discussion

CHAPTER FOUR: Histopathological Analysis of 3,3',4,4'-

Tetrachlorobiphenyl Toxicity in Multiple Organs of the Marine Fish Scup:

a Time-Course and Dose-Response Study
Abstract
Introduction
Materials and Methods
Results
Discussion

CHAPTER FIVE: In vitro Metabolism of Polychlorinated Biphenyl (PCB)

Congeners by the Marine Fish Scup (Stenotomus chrysops): Relationship to Cytochrome P450 Expression
Abstract
Introduction
Materials and Methods
Results
Discussion

CHAPTER SIX: In vitro Metabolism of Polychlorinated Biphenyl (PCB)

Congeners by Beluga Whale (Delphinapterus leucas) and Pilot Whale

(Globicephala melaena): Relationship to Cytochrome P450 Expression
Abstract
Introduction
Materials and Methods
Results
Discussion

CHAPTER SEVEN: Catalytic and Immunochemical Characterization of Hepatic Microsomal Cytochromes P450 in Beluga Whale (Delphinapterus leucas). Reprint from Toxicology and Applied Pharmacology 126.

Abstract

Introduction

Materials and Methods 
Results

Discussion

CHAPTER EIGHT: Summary and Conclusions

Teleost studies

Cetacean studies

BIBLIOGRAPHY 
Chapter 1:

Table 1: Selected Cytochrome P450 Gene Products

Chapter 2:

Table 1: Induction of Total Hepatic Cytochrome P450 due to CYP1A1

Table 2: Metabolites of 3,3',4,4'-TCB Covalently Bound to Microsomal Protein

Table 3: Cytochrome P450 Content and EROD Activity in Microsomal Incubations at 30 Minutes

Chapter 3:

Table 1: CYP1A1 Induction in Renal Tubules of TCB-Treated Scup

Table 2: CYP1A1 Induction in Multiple Cell Types on Day 5

Chapter 4:

Table 1: Number of Melanomacrophage Aggregates in Spleen of TCB-Treated and Control Fish

Table 2: Plasma Chemistry in Vehicle-Treated and TCB-Treated Fish

Table 3: TCB Concentration in Pooled Tissue Samples of TCB-Treated Scup

Chapter 5:

Table 1: Microsomal Enzyme Contents and Activities in Depurated, Feral, and Treated Scup

Table 2: Rate of Metabolism of ${ }^{14} \mathrm{C}$-TCB Congeners in Hepatic Microsomes from Feral Scup: Addition of a "Competing" TCB Congener

Chapter 6:

Table 1: Microsomal Monooxygenase Components and Activities in Pilot and Beluga Liver Samples

Table 2: TCB Metabolism Rates and P450 Parameters in Selected Samples

Chapter 7:

Table 1: Microsomal Electron Transport Components in Beluga Whale Liver

Table 2: Microsomal Monooxygenase Activities in Beluga Whale Liver

Table 3: Profile of Benzo[a]pyrene Metabolites Produced by Liver Microsomes of Beluga Whale

Table 4: Comparison of Liver Microsomal Monooxygenase Components and Activities in Different Species of Adult Cetacean 


\section{Chapter 2:}

Fig 1: Hepatic microsomal content of cytochrome P450 and cytochrome b5 40

Fig 2: Hepatic microsomal content of CYP1A1, P450B, and P450A 43

Fig 3: Hepatic microsomal EROD activity and PROD activity 45

Fig 4: Hepatic microsomal NADPH reductase activity and UGT activity 46

Fig 5: Northern blot of hepatic CYP1A1 mRNA in pooled scup samples 47

Fig 6: Hepatic CYP1A1 mRNA content 48

Fig 7: GC/MS analysis of TCB content in pooled liver samples 49

Fig 8: Spectrophotometric analysis of cytochrome P450 in microsomes 52

Fig 9: Enzyme inactivation by TCB in vitro 57

Fig 10: CYP1A1 inactivation in the presence of azide in vitro 59

Chapter 3:

Fig 1: Immunodetectable CYP1A1 content in kidney, gill, and liver 77

Fig 2: Rate of EROD activity in kidney, gill, and liver microsomes 79

Fig 3: IHC analysis of CYP1A1 induction in liver and kidney 83

Fig 4: Time-course of CYP1A1 induction in epithelial cells 85

Fig 5: Time-course of CYP1A1 induction in endothelial cells 87

Fig 6: IHC analysis of CYP1A1 induction in gill 91

Fig 7: IHC analysis of CYP1A1 induction in intestine and spleen 93

Fig 8: IHC analysis of CYP1A1 induction in heart and gonad 95

Chapter 4:

Fig 1: Cumulative mortality in treated fish $\quad 110$

Fig 2: Condition index and hepatosomatic index in treated fish 111

Fig 3: Histopathology in treated fish 113

Fig 4: H\&E stained slides of liver and spleen $\quad 115$

Fig 5: H\&E stained slides of kidney and gill 119

Fig 6: Scup tail fin $\quad 121$

Fig 7: GC/MS analyses of TCB content in pooled liver and muscle samples $\quad 126$

Chapter 5:

Fig 1: PCB congeners used in the study

Fig 2: Metabolism of TCB congeners in hepatic microsomes of feral scup $\quad 141$

Fig 3: Inhibition of 3,3',4,4'-TCB metabolism in feral scup 142

Fig 4: TCB / cytochrome P450 binding spectrum 144

Fig 5: XAD column profiles of 3,3',4,4'-TCB metabolites 146-147 


\section{Chapter 5:}

Fig 6: XAD column profiles of 2,2',5,5'-TCB metabolites

$148-149$

Fig 7: Rate of metabolism of TCB congeners in vitro versus immunodetectable P450 in scup hepatic microsomes

Fig 8: Mean rate of TCB metabolism versus mean content of immunodetectable cytochromes $\mathrm{P} 450$ in scup hepatic microsomes

Fig 9: Inhibition of alkoxyresorufin O-dealkylase activities by TCB congeners 158

Fig 10: Polyclonal antibody inhibition of metabolic activities in scup

Fig 1: Immunoblot of hepatic microsomes from pilot whale with MAb 1-12-3 176

Fig 2: Correlation between hepatic microsomal EROD activity and immunodetectable CYP1A content in pilot whales

Fig 3: SDS-PAGE immunoblots of cetacean liver microsomes with anti-CYP2B polyclonal antibodies

Fig 4: Rate of metabolism of 3,3',4,4'-TCB versus immunodetectable CYP1A content and EROD activity in cetacean liver microsomes

Fig 5: Inhibition of EROD activity in beluga microsomes by TCB and ANF

Fig 6: Inhibition of enzyme activities in beluga microsomes by ANF

Fig 7: XAD column profiles of 3,3',4,4'-TCB metabolites in beluga

Chapter 7:

Fig 1: Dithionite difference spectrum of beluga liver microsomes

Fig 2: Fluorescence spectra of PROD reaction mixtures

Fig 3: Immunoblot of beluga hepatic microsomes with MAb 1-12-3

Fig 4: SDS-PAGE immunoblot of beluga hepatic microsomes with monoclonal anti-scup P450E and polyclonal anti-mouse CYP1A1

Fig 5: Correlations of CYP1A1 content to length and to native cytochrome P450 196

Fig 6: Correlations between monooxygenase activity and CYP1A in beluga

Fig 7: SDS-PAGE immunoblots of beluga liver microsomes with antibodies against CYP2B forms

Fig 8: SDS-PAGE immunoblot of beluga liver microsomes with polyclonal anti-rat CYP2E1

Fig 9: Benzo[a]pyrene metabolite profile from a male beluga

Fig 10: CYP1A1 content in beluga liver microsomes versus concentration of non-ortho and mono-ortho PCBs in blubber samples of the same animals 


\section{ABBREVIATIONS}

Ah: aromatic hydrocarbon

AHH: aromatic hydrocarbon hydroxylase

ANF: $\quad \alpha$-naphthoflavone

BaP: benzo[a]pyrene

BNF: $\quad \beta$-naphthoflavone

BSA: bovine serum albumin

cDNA: complementary DNA

cpm/dpm: counts / disintegrations per minute

CYP: $\quad$ cytochrome P450

DCB: dichlorobiphenyl

DMSO: dimethyl sulfoxide

ECOD: ethoxycoumarin O-deethylase

7-ER: 7-ethoxyresorufin

EROD: ethoxyresorufin O-deethylase

HAH: halogenated aromatic hydrocarbons

HCB: hexachlorobiphenyl

H\&E: hematoxylin and eosin stain

HSI: hepatosomatic index

IHC: immunohistochemistry

IgG: immunoglobulin $\mathrm{G}$

ISF: isosafrole

IHC: immunohistochemistry

MAb: monoclonal antibody

3-MC: 3-methylcholanthrene

MCB: monochlorobiphenyl

MROD: methoxyresorufin O-demethylase

$\mathrm{NAD}(\mathrm{P}) \mathrm{H}$ : nicotinamide adenine dinucleotide (phosphate)

O.D.: $\quad$ optical density

pAb: polyclonal antibody

PAH: polycyclic aromatic hydrocarbons

PB: phenobarbital

PBS: $\quad$ phosphate buffered saline

PCB: $\quad$ polychlorinated biphenyl

PeCB: pentachlorobiphenyl 
ABBREVIATIONS, continued

PROD: pentoxyresorufin O-depentylase

SAR: $\quad$ structure-activity relationship

TCA: trichloroacetic acid

TCB: tetrachlorobiphenyl

TCDD: 2,3,7,8-tetrachlorodibenzo- $p$-dioxin

TCDF: $\quad$ 2,3,7,8-tetrachlorodibenzofuran

$\mu: \quad$ micro

$\mu \mathrm{Ci}: \quad$ microCurie

UGT: UDP-glucuronosyl transferase 


\section{CHAPTER ONE}

Introduction 


\section{Introduction}

The study of pollutant effects in marine animals is of broad interest to the biological sciences, firstly because responses in these organisms shed light on the health of the ecosystem, and secondly because these species serve as important models for pollutant effects on human health. Biological oceanography, which focuses on the interaction of marine organisms with their environment, must also consider the influence of anthropogenic chemicals in that environment, and how the presence of such compounds may affect life within the ocean. The world's oceans have increasingly become polluted with anthropogenic organic contaminants, including polycyclic aromatic hydrocarbons $(\mathrm{PAH})$ and halogenated aromatic hydrocarbons $(\mathrm{HAH})$. Even seemingly remote areas are now found to have detectable concentrations of these chemicals (Fowler, 1990). Marine animals may take up organic pollutants from all compartments of the ecosystem, including the sediments (benthic fish and invertebrates that live and feed at the bottom), the water column (via the gills in fish), and the other organisms (marine mammals and fish that feed near the top of food webs). Once internalized, organic contaminants may elicit a wide range of effects, from grossly visible tissue damage to subtle alterations in the levels of gene transcription. Perturbations thus occur at many levels, from the most fundamental to the most macroscopic: molecular, biochemical, cellular, histological, systemic, organismal, population effects, and ecosystem effects. There is a complex interplay between the toxic effects that are manifest at each level, and in many cases, changes detectable at one level may shed light on those that will occur at higher levels, or on those that have occurred at lower levels. This dissertation provides an integrated picture of the changes elicited at several levels by one environmental toxicant.

Central to the question of toxicant effect is the question of toxicant elimination, a means by which effects can be relieved. To some degree, organic contaminants may be eliminated in an unaltered form: in the urine and feces, in exchange across the gills (fish), in the eggs at spawning (fish), in parturition and lactation (mammals). However, many organic contaminants, including $\mathrm{PAH}$ and $\mathrm{HAH}$, are highly lipophilic and tend to partition into fat (Chiou, 1985), making their elimination particularly slow. In such cases, metabolism becomes an important route for elimination, particularly those metabolic pathways that generate products that are more hydrophilic than is the parent compound. One group of enzymes that achieves such metabolism in vertebrate animals is the cytochromes P450. This is a family of microsomal monooxygenase enzymes that acts upon an almost endless list of substrates, including endogenous compounds (e.g. steroids and fatty acids), naturally occurring, exogenous compounds (PAH and plant metabolites), 
and synthetic, exogenous compounds (HAH and many drugs). Cytochromes $\mathrm{P} 450$ act by binding to hydrophobic compounds and introducing a single oxygen atom, usually bonded to a substrate carbon atom, thus rendering the compound more water-soluble. Following the oxygenation of a xenobiotic substrate by P450, two pathways are possible: (1) the intermediate compound may undergo further metabolism by "Phase II" enzymes to generate a highly polar, (often) nontoxic product which is excreted from the cell or organism; or (2) the intermediate may form covalent adducts with cellular proteins (including P450 itself) or DNA, potentially resulting in toxicity, carcinogenesis, or mutagenesis. The fate of the xenobiotic compound is dependent upon the rate of formation of the oxygenated intermediates, the chemical stability of those intermediates, and the presence of Phase II enzymes (Nebert and Gonzalez, 1987). Cytochromes P450 are thus of crucial importance in determining the fate and effects of organic pollutants that are taken up by vertebrate animals. Thus, the study of the regulation of P450 in marine vertebrates is central to the understanding of their responses to environmental contaminants.

At the core of this dissertation is an investigation of the suite of interactions that occur between the HAH compound 3,3',4,4'-tetrachlorobiphenyl (TCB), and the microsomal monooxygenase enzyme cytochrome P4501A1 (CYP1A1). TCB is one of a class of compounds that binds to the cytosolic Ah (aromatic hydrocarbon) receptor in vertebrates, thus eliciting the induction of CYP1A proteins, as well as several other enzymes involved in xenobiotic metabolism (Nebert and Gonzalez, 1987). CYP1A proteins, in turn, act in the primary metabolism of TCB and other Ah receptor ligands. Thus TCB and CYP1A1 act to mutually regulate each other's levels in the organism. Studies conducted with whole-animal and cell culture systems from several vertebrate classes (teleost fish, mammals, birds) indicate that TCB and similar compounds, in substrate-binding to CYP1A proteins, cause decreased CYP1A enzyme activity and catalytic efficiency (inhibition). In addition, studies in a few species have indicated that exposure to increasing concentrations of TCB results in decreasing tissue concentrations of CYP1A protein or mRNA. Such an effect is intriguing, from a compound that has classically been identified as a CYP1A inducer. This issue of multi-leveled CYP1A "suppression" by TCB is of great interest to enzymology, as the mechanisms have been largely undescribed. But it is also an important question to biological oceanography, since chlorobiphenyls are a prevalent class of marine pollutant that are taken up by fish, and that can bring about toxic effects with potential repercussions at the population level. 


\section{Cytochrome P450 Structure and Function}

The cytochromes P450 are a family of heme-containing, monooxygenase enzymes that are found in the endoplasmic reticulum and mitochondria of most eukaryotic cell types. Various forms have been identified in species as diverse as animals, plants, fungi, and bacteria. The bacterial system is similar to that found in eukaryotic mitochondria and will not be addressed here. In the endoplasmic reticulum of eukaryotic cells, P450 is a terminal electron acceptor in the membrane-bound enzyme system depicted below (Peterson and Prough, 1986).

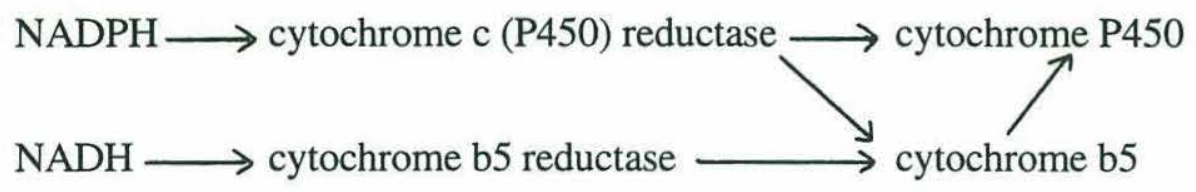

Along the primary path of electron flow, NADPH passes two electrons to a flavoprotein reductase, which contains both FAD and FMN prosthetic groups. The reductase then passes the electrons sequentially to the P450. In some cases, the second of the two electrons received by $\mathrm{P} 450$ is donated by cytochrome b5, which itself may be reduced by either reductase. The passage of electrons between reductase and P450 may be dependent upon the frequency of collisions of the two proteins within the membrane, and upon proper orientation of collisions. The rate of reduction of P450 is greatly enhanced when it is bound to a substrate (Ingelman-Sundberg, 1986).

The cytochrome $\mathrm{P} 450$ active site contains an iron protoporphyrin IX prosthetic group, which consists of one iron atom coordinated to four pyrrole rings. The iron is also coordinated to a cysteine residue of the surrounding protein by a thiolate ligand (Black and Coon, 1986), and a sixth iron coordination site is available for interaction with oxygen or various substrates and inhibitors. The heme group is surrounded by hydrophobic amino acids which comprise the substrate binding domain of the enzyme. The enzyme is buried in the phospholipid membrane of the endoplasmic reticulum, with some parts exposed on the cytoplasmic face. The phospholipids act to facilitate the P450/reductase association, help to maintain the conformation of the P450, and influence the enzyme/substrate binding interaction (Ingelman-Sundberg, 1986). It has been demonstrated that cytochromes P450 carry out stereoselective oxidation of their substrates, which suggests a specific substrate orientation within the active site during catalysis. 
The catalytic cycle of cytochrome P450 is illustrated below, with Fe representing the heme iron in the cytochrome P450 active site. The cycle proceeds as follows (McMurry and Groves, 1986; Ortiz de Montellano, 1986):

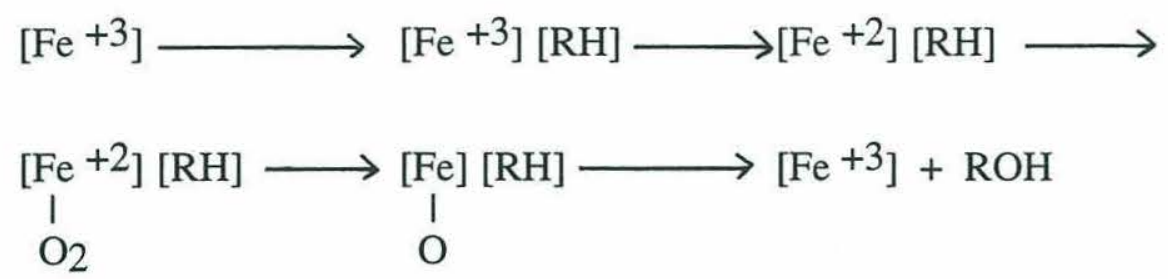

(1) substrate binding to enzyme, by hydrophobic interactions with the protein surrounding the heme, to give a high-spin ferric complex; (2) transfer of one electron from reductase, reducing the iron to the ferrous form; (3) covalent binding of molecular oxygen to the iron atom in the heme active site, to generate a ferrous P450-dioxygen complex; (4) transfer of a second electron from reductase or b5 to the heme iron; (5) cleavage of the dioxygen bond, with incorporation of the distal oxygen into a water molecule; (6) transfer of the second oxygen atom from iron to the substrate; (7) dissociation of the oxygenated product from the enzyme, regenerating the ferric low-spin state of the enzyme. Reactions that are carried out by the above scheme include hydrocarbon hydroxylation, olefin epoxidation, aromatic hydroxylation (often preceded by epoxidation), nitrogen and sulfur oxidation, and $\mathrm{O}$ dealkylation of ethers and other oxygen derivatives. These transformations are involved in the metabolism and biosynthesis of steroids, as well as the metabolism of xenobiotics, including drugs, plant products, combustion products, and environmental contaminants. In addition, cytochrome P450 can catalyze substrate-independent autooxidation reactions, in which bound oxygen is converted to either one molecule of hydrogen peroxide or two molecules of water.

The superfamily of cytochromes P450 is comprised of a number of distinct gene products, with broad and overlapping substrate specificities. These enzymes are capable of binding and oxygenating a vast array of xenobiotic and physiological compounds, although those involved in biosynthetic pathways generally display more narrow substratespecificities than those that are devoted to xenobiotic metabolism. At least 221 different P450 genes have been identified, in 36 gene families, as defined by percent similarity of protein sequence (Nelson et al., 1993). It is theorized that four of these families (P4501 P4504) have evolved in animals in response to exposure to plant metabolites and decayed plant products (Nebert and Gonzalez, 1987). Evidence for this theory lies in the finding that these four families are involved in xenobiotic metabolism, and that many xenobiotic 
substrates (including some drugs and combustion products) are plant-derived.

Physiological functions have been ascribed to some genes in these four families, but for others such as CYP1A1, no physiological function is yet known. Table 1 provides a partial list of these gene products, including some known inducers (Nebert and Gonzalez, 1987; Stegeman, 1989; Nelson et al., 1993; Savas et al., 1994).

TABLE 1: Selected Cytochrome P450 Gene Products

\begin{tabular}{llll}
\hline subfamily & protein & trivial names & regulation \\
\hline P4501A & CYP1A1 & rat c, rat BNF-B, & PAH, planar PCB, \\
& mouse P1 & TCDD \\
& CYP1A2 & rat d, rat ISF-G, & PAH, planar PCB, \\
& mouse P3 & TCDD, ISF \\
P4501B & CYP1B1 & rat EF & PAH, TCDD \\
P4502A & CYP2A1 & rat a & PAH, planar PCB \\
P4502B & CYP2B1 & rat b, rat PB-B & PB, non-planar PCB \\
& CYP2B2 & rat e & PB, non-planar PCB \\
P4502C & CYP2C7 & rat $f$ & PB, constitutive \\
P4502D & CYP2D1 - 2D5 & rat db1 - db5 & constitutive \\
P4502E & CYP2E1 & rat j & ethanol, starvation \\
P4503A & CYP3A1 - 3A2 & rat pcn1 - pcn2 & steroids, PB \\
P4504A & CYP4A1 & rat LA $\omega$ & clofibrate \\
\hline
\end{tabular}

\section{Cytochrome P450 Regulation}

Cytochromes P450 fall into two groups with distinct regulatory mechanisms (Waterman et al., 1986): those forms that metabolize physiological or endogenous substrates are generally regulated endogenously, with constitutive levels determined by cellular signaling chemicals such as peptide hormones; those forms that metabolize xenobiotic or exogenous substrates are largely modulated by substrate induction. Induction commonly occurs via an enhanced transcription of the P450 gene, but in many cases also involves a post-transcriptional component, such as enhanced stabilization of existing P450 protein (e.g. CYP2E1; Song et al., 1986). The division of P450 enzymes into two categories is not so clear-cut, however, for many inducible enzymes are also regulated by 
endogenous factors. For example, mammalian CYP1A and CYP2B are inducible by exogenous $\mathrm{PAH}$ and $\mathrm{PB}$, respectively, and are also under endogenous regulation which determines level of transcriptional activity at different stages of development (Nebert and Gonzalez, 1987). Endogenous regulation results in enzyme levels that vary with developmental stage, sex, and cell or tissue type.

The regulation of CYP1A enzymes is currently the best characterized of all inducible P450s. Induction occurs principally at a transcriptional level, upon exposure to Ah receptor ligands, many of which are also CYP1A substrates. The current model describing the process is as follows (Nebert and Gonzalez, 1987; Stegeman and Hahn, 1994): (1) the ligand crosses the plasma membrane into the cell, and binds with the cytosolic Ah (aromatic hydrocarbon) receptor; (2) a heat shock protein (hsp90) dissociates from the Ah receptor upon ligand binding, and the ligand/receptor complex then translocates to the nucleus, which involves dimerization with the Ah receptor nuclear translocator (ARNT) protein; (3) the ligand/receptor complex binds to specific sequences of DNA identified as xenobiotic responsive elements (XREs), resulting in transcriptional activation of genes encoding CYP1A1, CYP1A2, and several Phase II metabolic enzymes; (4) enhanced CYP1A mRNA concentrations bring about increased production of CYP1A protein(s); (5) these proteins may then bind and/or metabolize the ligand molecules. Organic xenobiotic compounds thus promote their own metabolism, which may activate them to more toxic or carcinogenic forms, and may effect their detoxification and elimination. The degree of induction is a function of both inducer concentration and inducer efficacy. Higher concentrations often result in greater induction, but exceptions to this have been observed (for example, PCBs discussed below). The most potent CYP1A inducer yet described is 2,3,7,8-tetrachlorodibenzo- $p$-dioxin (TCDD), which has been found to be 10,000 times more potent an inducer of monooxygenase activity than 3methylcholanthrene in mouse (Poland and Glover, 1974).

\section{Cytochrome P450 in Marine Vertebrates}

Many P450 enzymes have been identified in several fish species, including rainbow trout, scup, cod, perch, plaice, and little skate (Stegeman and Hahn, 1994). Several teleost forms are thought to be homologous with inducible mammalian P450 enzymes, including CYP1A, CYP2B, CYP2E, CYP3A, and CYP4A. Such assignments are based upon immunochemical cross-reactivity, similarity of catalytic function, and in some cases sequence similarity. Other fish P450s have been found to be present constitutively, and some are known to serve specific physiological functions such as steroid metabolism. 
P450E from the marine fish scup (Stenotomus chrysops) has recently been sequenced, and was determined to be a CYP1A1 form (Morrison et al., submitted). Studies of the regulation of teleost CYP1A1 have indicated many similarities to regulation in mammals. Exposure to Ah-receptor agonists results in an increased level of CYP1A1encoding mRNA, which is followed in time by increased CYP1A1 protein content and catalytic activity (Kloepper-Sams and Stegeman, 1989). Ah receptor has been identified in several species of bony fish (Hahn et al., 1994), indicating that CYP1A1 induction in these species is likely mediated by this receptor, as it is in mammals. CYP1A1 is induced in fish by many of the same compounds that induce mammalian CYP1A1, including high molecular weight PAH and coplanar PCB, but unlike mammalian CYP1A1, is apparently not induced by mono-ortho-substituted PCB (Gooch et al., 1989). Questions concerning CYP1A1 induction in fish are addressed in chapters 2 and 3 of this thesis.

$\mathrm{P} 450 \mathrm{~B}$ from the marine fish scup is believed to be homologous to members of the mammalian CYP2B subfamily, based upon immunochemical cross-reactivity of scup P450B and rat CYP2B1 (Stegeman et al., 1990a; Stegeman et al., 1990b). Furthermore, the N-terminal amino acid sequence of $\mathrm{P} 450 \mathrm{~B}$ is about $50 \%$ identical to the same region of rat CYP2B1/2 (Stegeman and Hahn, 1994). The mechanism of regulation of the putative teleost CYP2B is not yet known. Treatment of fish with known inducers of mammalian CYP2B has consistently failed to bring about increased levels of microsomal activities that are catalyzed by mammalian CYP2B (reviewed in chapter 5). It is not yet known whether fish CYP2B responds to a different suite of inducers than do the mammalian forms, or whether fish CYP2B is not inducible by exogenous compounds. Such questions will require further investigation, and are addressed briefly in chapter 5 of this thesis.

Far less is known about cytochromes P450 in marine mammals. P450 systems have been identified and partially characterized in a few cetacean species, namely minke whale (Goksøyr et al., 1986; Goksøyr et al., 1988), short-finned pilot whale, killer whale, striped dolphin (Watanabe et al., 1989), beluga whale (White et al., 1994), and long-finned pilot whale (chapter 6). Proteins have been detected in liver microsomes from minke whale, beluga whale, and long-finned pilot whale by cross-reaction with antibodies raised against CYP1A1 (Goksøyr et al., 1988; Goksøyr et al., 1989; White et al., 1994). All cetacean species examined catalyzed EROD, ECOD, and AHH activities, which are characteristic of CYP1A in mammals. Thus it is fairly certain that cetaceans express a CYP1A homologue, which appears to be inducible by exposure to coplanar PCB (White et al., 1994). It is unclear, however, whether cetaceans express a CYP2B homologue that is similar to CYP2B in terrestrial mammals. Catalytic activities characteristic of mammalian CYP2B are consistently low in cetacean samples, including aldrin epoxidase activity 
(Watanabe et al., 1989), PROD activity (White et al., 1994), and metabolism of 2,2',5,5'-

TCB (chapter 6). Immunochemical studies with antibodies raised against rat CYP2B1/2B2 have detected no cross-reacting proteins in minke whale (Goksøyr et al., 1989), beluga whale (White et al., 1994), or long-finned pilot whale (chapter 6). It is possible that cetaceans do not express a protein homologous to mammalian CYP2B, consistent with the divergent evolution of terrestrial and marine mammals. Questions concerning CYP1A and CYP2B expression and activity in cetaceans are addressed in chapters 6 and 7 of this thesis.

\section{Polychlorinated Biphenyls: Distribution, Toxicity, Metabolism}

Polychlorinated biphenyls (PCBs) are a group of 209 compounds composed of a pair of aromatic rings, joined by a single carbon-carbon bond, with various patterns of chlorine substitution on the remaining 10 carbon atoms. Positions of chlorine substitution have been numbered $2-6$ on one ring and $2^{\prime}-6^{\prime}$ on the second ring, which are also referred to as the ortho, meta, and para positions of substitution, with respect to the central bond (see diagram below).

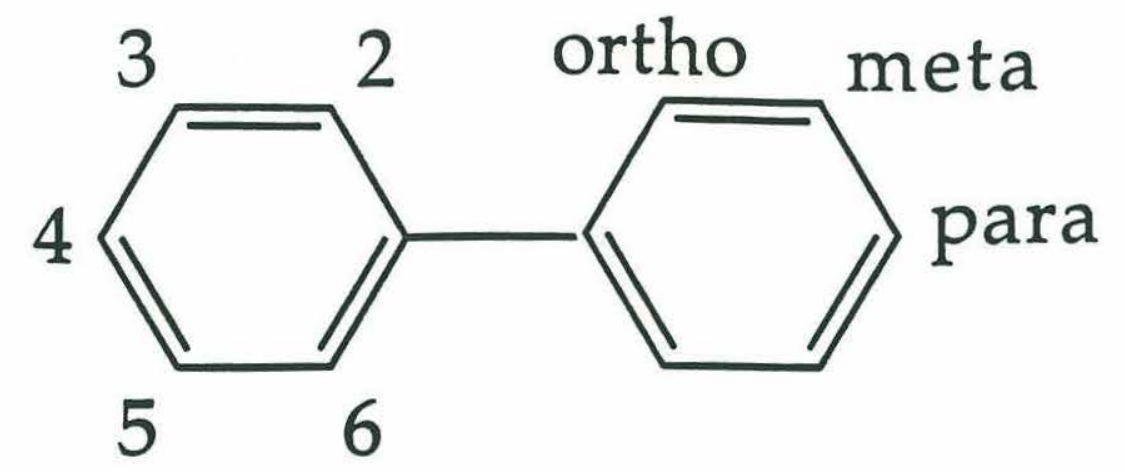

PCBs are entirely synthetic, first created in 1881 , and were used heavily by the electrical industry from the 1940 s to the 1970 s, thus leading to their global distribution. Widespread, environmental contamination by PCBs was first discovered in 1966 by a Swedish scientist (Jensen, 1966), and later by a group of Americans (Risebrough et al., 1968), in analysis of wildlife and human specimens. Since these early discoveries, PCBs have been detected throughout the globe, in water, soil, sediment, biota, and air (Tanabe, 1988; Fowler, 1990). Of the total volume of PCBs ever synthesized, it is estimated that 
$31 \%$ has been released into the environment, $65 \%$ is "land-stocked" (either still in use in electrical equipment or deposited in landfills), and $4 \%$ has been degraded or incinerated (Tanabe, 1985). Of the $31 \%$ that comprises the environmental load, it is estimated that $97 \%$ is retained in coastal sediments and open ocean water.

Adverse human health effects of PCBs were first reported as early as the 1930s, in exposed industrial workers (Schwartz, 1936). Early reported conditions included "yellow atrophy of the liver" and "dermatitis" (Schwartz, 1936; Collier, 1943; Schwartz, 1943). Extensive work since that time has identified PCBs as neurotoxic, genotoxic, hepatotoxic, immunotoxic, and toxic to reproductive systems in mammals (Safe, 1984; Safe, 1992). Far less is known about PCB toxicity in teleost species, but effects are thought to include growth inhibition, impaired bone development, reproductive impairment (McFarland and Clark, 1989), and immune suppression (Spitsbergen et al., 1988c). PCB toxicity and histopathology in fish is addressed in chapter 4 of this thesis.

In laboratory mammals, the oxidative metabolism of PCBs is catalyzed primarily by cytochromes P450. Structure-activity relationships appear to govern the metabolism of PCBs by $\mathrm{P} 450$, with chlorine position determining which P450 enzyme carries out the transformation. In rat liver, hydrocarbon-inducible CYP1A forms metabolize congeners with halogenated substituents at one or both para positions, and with adjacent unsubstituted ortho-meta carbons, while phenobarbital-inducible CYP2B forms (CYP2B1, CYP2B2) metabolize congeners with adjacent, unsubstituted meta-para positions (Kaminsky et al., 1981; Kennedy et al., 1981; Shimada and Sawabe, 1983; Mills et al., 1985; Ishida et al., 1991). In addition, it was observed that PCB congeners with two or more ortho

substituents are more rapidly metabolized by rat CYP2B than by CYP1A, while congeners lacking ortho substituents are more rapidly metabolized by rat CYP1A than by CYP2B. There is currently no direct information concerning the role of cytochromes P450 in PCB metabolism in fish, or in marine mammals. These issues are addressed in chapters 5 and 6 of this thesis, respectively.

\section{Interaction Between Polychlorinated Biphenyls and Cytochromes P450}

PCBs were first identified as inducers of cytochrome P450 in 1973 (Alvares et al., 1973), in a study that suggested that PCB mixtures may induce both "PB-inducible" (P450) and "MC-inducible" (P448) proteins. Later investigations of individual PCB congeners were able to determine "structure activity rules" for the induction of various P450 forms by the different PCB congeners in rat (Parkinson et al., 1980; Parkinson et al., 1983). These studies found the following: the coplanar PCB congeners (lacking ortho 
substituents) induced CYP1A1, CYP1A2, and CYP2A1; the PCB congeners with two or more ortho substituents induced CYP2B1 and CYP2B2; the mono-ortho substituted $\mathrm{PCB}$ congeners induced all five of these P450 enzymes, thus earning the name "mixed-type" inducers. Induction patterns in fish have proved to be somewhat different. As discussed above, no chemical has yet been found to induce the putative CYP2B form in fish. The coplanar PCB congeners were found to be potent inducers of CYP1A1 in scup, but those congeners with one or two ortho substituents induced neither CYP1A1 nor total P450 content (Gooch et al., 1989).

The coplanar PCB congeners, in addition to acting as inducers of CYP1A enzymes, have also been shown to inhibit CYP1A activity in vivo and in vitro. These congeners include 3,3',4,4'-tetrachlorobiphenyl (TCB, IUPAC \#77), 3,3',4,4',5pentachlorobiphenyl (PeCB, IUPAC \#126), and 3,3',4,4',5,5'-hexachlorobiphenyl (HCB, IUPAC \#169). As increasing doses of coplanar PCB are administered, decreasing rates of hepatic CYP1A enzyme activity and decreasing catalytic efficiencies have been observed. This has been found in fish (Melancon and Lech, 1983; Miranda et al., 1990; Monosson and Stegeman, 1991; Lindstrom-Seppa et al., 1994), rat (Voorman and Aust, 1988), and in cultured hepatocytes from fish (Hahn et al., 1993), rat (Sawyer and Safe, 1982), and bird (Rodman et al., 1989; Kennedy et al., 1993). The latter studies have not found, or have not reported, a decrease in CYP1A protein content to accompany the decrease in rate of CYP1A enzyme activity. Several in vitro studies have indicated that TCB and HCB act as competitive or mixed inhibitors of CYP1A activity in hepatic microsomes or in reconstituted systems (Voorman and Aust, 1987; De Matteis et al., 1989; Gooch et al., 1989). The reduced CYP1A activity in the aforementioned studies, without a drop in CYP1A protein content, is likely due to such inhibition. This would involve the residual $\mathrm{PCB}$ in hepatic microsomes binding to the CYP1A active site, and inhibiting the binding and catalysis of the substrate of interest.

Other investigators have reported a decline in rate of CYP1A enzyme activity, accompanied by a drop in the content of CYP1A protein, when higher doses of TCB were administered. These studies have included work with fish (Gooch et al., 1989), cultured bird hepatocytes (Sinclair et al., 1984; Lambrecht et al., 1988; Sinclair et al., 1989; Lorenzen and Kennedy, in preparation), and cultured porcine aorta endothelial cells (Stegeman et al., in press). Still other investigators have observed reduced expression of CYP1A mRNA, in fish exposed to higher TCB doses as compared to those exposed to lower total doses (Wirgin et al., 1992; Celander and Forlin, submitted). The mechanisms by which TCB elicits down-regulation of CYP1A protein and mRNA are largely unknown, and are the subject of investigation of chapter 2 in this thesis. 


\section{Goals of Dissertation Research}

The disruption of CYP1A1 functioning by TCB, as described above, may pose a serious threat to the health of marine fish, for the CYP1A1 enzyme system appears to play a critical role in the metabolism and detoxification of organic contaminants in these species. The central goal of the dissertation work was thus to describe the mechanisms by which TCB can regulate CYP1A1 expression, content, and activity in the marine fish scup (Stenotomus chrysops). A long-term, dose-response study was conducted, in which groups of fish were treated with either a low (strongly inducing) TCB dose or a high (strongly inhibitory) TCB dose. Samples from this study were employed to address several questions (chapters 2, 3, and 4).

\section{Chapter 2:}

(1) What is the time-course of the response of hepatic CYP1A1 to $0.1 \mathrm{mg} / \mathrm{kg}$ TCB versus $5.0 \mathrm{mg} / \mathrm{kg} \mathrm{TCB}$, in terms of CYP1A1 mRNA content, CYP1A1 protein content, and CYP1A1 catalytic activity?

(2) What is the range of hepatic TCB concentrations that are associated with CYP1A1 induction; what is the range of hepatic TCB concentrations that are associated with CYP1A1 inhibition and/or suppression?

(3) Are the inhibition of activity and suppression of protein content by the high TCB dose specific for CYP1A1, or general to some group of hepatic proteins?

(4) At what level(s) of control does TCB exert a negative effect on CYP1A1:

transcription, mRNA stability, translational, protein stability, enzyme activity?

(5) What are the mechanism(s) by which TCB exerts a negative effect on CYP1A1?

\section{Chapter 3:}

(1) What is the level of CYP1A1 protein content in various organs and cell types, following treatment of fish with $0.1 \mathrm{mg} / \mathrm{kg}$ TCB or $5.0 \mathrm{mg} / \mathrm{kg}$ TCB?

(2) Does the level of CYP1A1 expression vary with TCB dose in the extrahepatic organs?

(3) What organs and what cell types show suppressed CYP1A1 content at the high TCB dose?

\section{Chapter 4:}

(1) Does treatment with $0.1 \mathrm{mg} / \mathrm{kg}$ TCB or $5.0 \mathrm{mg} / \mathrm{kg}$ TCB elicit histopathology or toxicity in scup? 
(2) What organs and cell types are targeted for pathology by TCB?

(3) Can histopathology explain the suppressed CYP1A1 content caused by a high TCB dose?

A series of in vitro studies was designed, to further explore the role of CYP1A1 in the disposition of $3,3^{\prime}, 4,4^{\prime}-$ TCB. Studies were also included to explore the role of the putative scup CYP2B in the disposition of 2,2',5,5'-TCB.

\section{Chapter 5:}

(1) Can scup metabolize 3,3',4,4'-TCB and 2,2',5,5'-TCB to aqueous-soluble products in vitro?

(2) What role do scup CYP1A1 and scup CYP2B play in the oxidative metabolism of these PCB congeners in vitro?

\section{Chapter 6:}

(1) Can hepatic microsomes from beluga whale (Delphinapterus leucas) and pilot whale (Globicephala melaena) metabolize 3,3',4,4'-TCB and 2,2',5,5'-TCB to aqueous-soluble metabolites?

(2) What role does cetacean CYP1A play in these activities?

(3) Do cetaceans express enzymes homologous to mammalian CYP2B forms?

\section{Chapter 7:}

This is a published investigation of the hepatic cytochrome P450 enzyme system in the beluga whale (Delphinapterus leucas). 


\section{CHAPTER TWO}

3,3',4,4'-Tetrachlorobiphenyl Elicits Induction,

Inhibition, and Inactivation of Cytochrome P4501A1 in

Teleost Liver in vivo and in vitro 


\begin{abstract}
The polychlorinated biphenyl congener 3,3',4,4'-tetrachlorobiphenyl (TCB) is a known inducer of cytochrome P4501A (CYP1A) enzymes in vertebrates, but it has recently been recognized as an inhibitor of CYP1A as well. Evidence also suggests that it may inactivate or down-regulate CYP1A, but the mechanisms of such inactivation and down-regulation have been largely unknown. We investigated TCB action by treating the marine fish scup
\end{abstract} (Stenotomus chrysops) by intraperitoneal injection with two doses of TCB, $0.1 \mathrm{mg} / \mathrm{kg}$ and $5.0 \mathrm{mg} / \mathrm{kg}$, and sampling groups of animals over a 16 day time-course. The low dose of TCB brought about a strong induction of hepatic CYP1A1 mRNA (20-fold), protein (12fold), and catalytic activity (15-fold), with maxima on days 2, 5, and 5, respectively, at levels sustained for 12 days. Similarly, TCB concentration in the livers of these animals was maximal on day 5 and declined thereafter. The high dose of TCB caused an induction of CYP1A1 mRNA equal to that with the low dose, but CYP1A1 protein content was only weakly induced (4-fold), and rates of CYP1A1 activity were near control levels, from day 2 through 12. By day 16, however, the levels of CYP1A1 protein and activity in animals receiving the high TCB dose were similar to those in animals treated with the low dose. This increase on day 16 corresponded to a reduced hepatic TCB content by day 16 , in the fish treated with $5.0 \mathrm{mg} / \mathrm{kg}$. The two TCB doses caused similar, slight elevations in expression of cytochrome b5 (2-fold), NADPH cytochrome c (P450) reductase (0.5-fold), and UDP-glucuronosyl transferase (2-fold). Neither dose had a strong influence on hepatic content of microsomal cytochrome P450B (a putative CYP2B form) or P450A (a putative CYP3A form). These in vivo data indicate that high hepatic concentrations of TCB act at post-transcriptional level to cause a specific reduction in CYP1A1 protein content. Incubation of scup hepatic microsomes with TCB plus NADPH caused a time-dependent loss of CYP1A1 catalytic capacity and a simultaneous conversion of P450 to the inactive form P420 (50\% in 30 minutes); neither effect was seen in the absence of cofactor. The rate of production of reactive TCB metabolites was slight, suggesting a possible role for mechanism-based CYP1A1 inactivation in vivo, but the rate was too slow to account for the inactivation and denaturation seen in vitro. The addition of azide to microsomal incubations containing TCB and NADPH caused a complete inactivation of microsomal CYP1A1 within 10 minutes. Azide is an inhibitor of catalase activity, and is also known to form azidyl radicals in the presence of hydroxyl radical, thus azide-enhanced inactivation of CYP1A1 suggests the production of reactive oxygen species by CYP1A1. The in vitro data indicate that TCB occupation of the CYP1A1 active site elicits enhanced production of reactive oxygen species, which appear to play a role in CYP1A1 inactivation in vitro and may play a role in reduced CYP1A1 content in vivo. 


\section{Introduction}

3,3',4,4'-Tetrachlorobiphenyl (TCB) has long been recognized as a potent inducer of cytochrome P4501A (CYP1A) enzymes in vertebrate species, including mammals (Parkinson et al., 1980; Parkinson et al., 1983) and fish (Gooch et al., 1989). TCB is a component of commercial polychlorinated biphenyl (PCB) mixtures, which are widespread environmental contaminants that have been found to elicit reproductive damage, as well as neurotoxic, genotoxic, hepatotoxic, and immunotoxic effects in mammals (reviewed in Safe, 1984; Safe, 1992). The coplanar PCB congeners, 3,3',4,4'-tetrachlorobiphenyl (TCB, IUPAC \#77), 3,3',4,4',5-pentachlorobiphenyl (PeCB, IUPAC \#126), and $3,3^{\prime}, 4,4^{\prime}, 5,5^{\prime}$-hexachlorobiphenyl (HCB, IUPAC \#169) are Ah receptor agonists in mammals and elicit a suite of toxic effects similar to those elicited by $2,3,7,8-$ tetrachlorodibenzo-p-dioxin. Ah receptor has been identified in fish species (Hahn et al., 1994), thus CYP1A induction in fish, by TCB and other aromatic hydrocarbons, is likely mediated by ligand binding to this receptor. In turn, CYP1A is involved in the primary metabolism of TCB in both mammals (Shimada and Sawabe, 1983; Mills et al., 1985; Ishida et al., 1991) and fish (chapter 5).

Depending upon the dose administered, TCB has been found to induce, inhibit, and/or suppress hepatic CYP1A in vivo. At relatively low doses (e.g. $0.1 \mathrm{mg} / \mathrm{kg}$ ), TCB brings about a strong, transcriptional induction of CYP1A1 in fish (Gooch et al., 1989). When higher doses of TCB or the other coplanar congeners (PeCB, HCB) are administered, several investigators have noted decreased rates of hepatic CYP1A enzyme activity, as compared to animals receiving lower doses. This observation has been made in fish (Melancon and Lech, 1983; Miranda et al., 1990; Monosson and Stegeman, 1991; Lindstrom-Seppa et al., 1994), rat (Voorman and Aust, 1988), and in cultured hepatocytes from fish (Hahn et al., 1993), rat (Sawyer and Safe, 1982), and bird (Rodman et al., 1989; Kennedy et al., 1993). The latter studies have not found, or have not reported, a decrease in CYP1A protein content to accompany the decrease in rate of CYP1A enzyme activity. Several in vitro studies have indicated that TCB and $\mathrm{HCB}$ act as competitive or mixed inhibitors of CYP1A activity in hepatic microsomes or in reconstituted systems (Voorman and Aust, 1987; De Matteis et al., 1989; Gooch et al., 1989). The loss of CYP1A activity in the aforementioned studies, without a drop in CYP1A protein content, is likely due to such inhibition. This would involve the residual PCB in hepatic microsomes binding to the CYP1A active site, and inhibiting the binding and catalysis of the substrate of interest.

Other investigators have reported a decline in rate of CYP1A enzyme activity, accompanied by a drop in the content of CYP1A protein, when higher doses of TCB were 
administered. These studies have included work with fish (Gooch et al., 1989), cultured bird hepatocytes (Sinclair et al., 1984; Lambrecht et al., 1988; Sinclair et al., 1989; Lorenzen and Kennedy, in preparation), and cultured porcine aorta endothelial cells (Stegeman et al., in press). Still other investigators have observed reduced expression of CYP1A mRNA, in fish exposed to higher TCB doses as compared to those exposed to lower total doses (Wirgin et al., 1992; Celander and Forlin, submitted). The mechanisms by which TCB elicits down-regulation of CYP1A protein and mRNA are largely unknown.

In the present study we have sought to characterize the specificity and mechanisms of CYP1A1 down-regulation by TCB. We describe the time-course of CYP1A1 response to two different doses of TCB, and relate CYP1A1 content to TCB concentration in liver. We report that relatively low hepatic TCB concentrations are associated with a strong, transcriptional induction of CYP1A1, whereas higher hepatic TCB concentrations bring about a much weaker induction of CYP1A1 protein and enzyme activity, even though expression of CYP1A1 mRNA remains high. This down-regulation appears to be specific to CYP1A1, and not general to microsomal enzymes, or even to cytochromes P450. In vitro studies suggest that suppressed CYP1A1 protein content is a result of oxidative inactivation of the enzyme, occurring during TCB occupation of the CYP1A1 active site.

\section{Materials and Methods}

Chemicals 3,3',4,4'-Tetrachlorobiphenyl (TCB, IUPAC \#77) was purchased from Pathfinder (St. Louis, MO). Purity of TCB was greater than $99 \%$ by GC/ECD and GC/MS analysis. Further analysis by high resolution (5000 resolution) mass spectrometry (VG Autospec) and selective ion monitoring showed that the potent Ah receptor agonist 3,3',4,4',5-pentachlorobiphenyl was not a contaminant of the TCB, with a detection limit of $0.001 \%$. Ethoxyresorufin and pentoxyresorufin were from Molecular Probes (Eugene, OR). All other chemicals used were of the highest grade available from commercial suppliers, and were similar to those used previously in this laboratory.

Animals and dissection Scup (Stenotomus chrysops) were caught by angling in Vineyard Sound, MA in August, 1991. Fish were held in flowing seawater tanks at $20^{\circ} \mathrm{C}$ for fourteen months prior to experimental use, and were maintained on a diet of Purina trout chow and frozen squid. Prior studies in our laboratory indicate that the bulk of environmental contaminants are depurated from the tissues of fish that are held in aquaria for one year, and that these fish show little CYP1A1 expression. At the time of the dosing, experimental animals (mixed sex) were gonadally immature and weighed 76 - 276 grams. 
Individuals were injected intraperitoneally with either corn oil (carrier controls) or with 3,3',4,4'-tetrachlorobiphenyl (TCB) dissolved in corn oil, at either $0.1 \mathrm{mg}$ TCB/ kg body weight or $5.0 \mathrm{mg} \mathrm{TCB} / \mathrm{kg}$ body weight. A group of uninjected fish was used as a "zero" point in the time-course. Individuals were held in flowing seawater tanks at $20^{\circ} \mathrm{C}$ until sampling (1 day to 18 days) or until death from toxicity. Fish were fed Purina trout chow once weekly, and were never fed within the three days prior to killing. At each time point, a group of 4 or 5 animals from each treatment group were killed by cervical transection and dissected immediately. Liver and muscle sections for GC analysis were rinsed in methanol and stored in acid-washed vials at $-20^{\circ} \mathrm{C}$ until analysis. Liver sections for RNA preparation and microsome preparation were removed and frozen in liquid nitrogen within one to two minutes of death. Tissues remained in liquid nitrogen until microsome preparation or RNA isolation. Prior studies in our laboratory indicate that enzyme degradation does not occur to any appreciable degree in scup tissue stored at liquid nitrogen temperatures (unpublished observations). Hepatic microsomal fractions were prepared by differential centrifugation, as previously described (Hahn and Stegeman, 1994), and microsomal protein content was measured with the bicinchoninic acid method (Smith et al., 1985), using bovine serum albumin as a standard. None of the treatments caused a change in hepatosomatic index, gonadosomatic index, or in a morphometric index of body weight to surface area (data in chapter 4). Microsomal yield ranged from 6 to $28 \mathrm{mg}$ endoplasmic reticulum/ $\mathrm{g}$ liver, and was not different between the various treatment groups.

Hepatic microsomes from feral scup were used for in vitro studies. These animals were caught by angling in Tarpaulin Cove, MA in the summer of 1988. Animals were killed immediately upon capture, and livers were stored in liquid nitrogen until microsome preparation. Microsomes were prepared from pooled samples, consisting of livers from 20 - 70 fish each.

Monooxygenase system The content of hepatic microsomal cytochrome P450 was determined from dithionite difference spectra of CO-treated samples (Stegeman and Binder, 1979). Cytochrome b5 was measured in liver microsomes using NADH difference spectra, as previously described (Stegeman and Binder, 1979).

Immunoblotting Western blot analysis of microsomal samples was performed as described previously (Kloepper-Sams et al., 1987; Stegeman et al., 1991). The three primary antibodies used were raised against the three principal P450 forms isolated from scup liver: monoclonal antibody 1-12-3 (Park et al., 1986), raised against scup P450E (a CYP1A1 homologue); polyclonal 7-94, raised against scup P450B (a likely CYP2B homologue; 
Stegeman et al., 1990a), and polyclonal 7-93, raised against scup P450A (a possible CYP3A homologue; Celander et al., 1994). Each antibody forms a strong reaction with its own immunogen in Western blots, and each forms negligible cross-reactions with the other two scup P450 forms (Kloepper-Sams et al., 1987; Gray, unpublished). Secondary antibodies used were alkaline phosphatase linked goat-anti-mouse IgG and goat-anti-rabbit IgG. Relative content of each protein in the microsomal samples was measured by densitometric scanning of immunoreactive bands with a video imaging system (Masterscan, Scanalytics, division of CSPI, Billerica, MA). In the case of P450E, protein was quantified by comparison of optical density in the samples to optical density in standards with known P450E content.

Enzyme assays Ethoxyresorufin O-deethylase (EROD) activity was measured in hepatic microsomes at $32^{\circ} \mathrm{C}$ with a fluorometric kinetic assay, using a Cytofluor 2300 fluorescence plate reader (Millipore), as described previously (Hahn et al., 1993). Pentoxyresorufin Odepentylase (PROD) activity was measured similarly, but substrate was used at $5 \mu \mathrm{M}$ nominal concentration rather than $2 \mu \mathrm{M}$ (as for EROD). NADPH-cytochrome c (P450) reductase activity was measured at $30^{\circ} \mathrm{C}$ by monitoring absorbance at $550 \mathrm{~nm}$, as described previously (Stegeman et al., 1979). UDP-glucuronosyl transferase activity (UGT) was measured in liver microsomes at room temperature, according to published procedures (Andersson et al., 1985). The assay contained 0.3 $\mathrm{M} \mathrm{KH}_{2} \mathrm{PO}_{4}$ (pH 7.0), $1.3 \mathrm{mg} / \mathrm{ml}$ digitonin detergent, $0.2 \mathrm{mM}$ p-nitrophenol as substrate, $2.7 \mathrm{mg} / \mathrm{ml}$ UDPGA (omitted from blanks), and $1.1-3.7 \mathrm{mg} / \mathrm{ml}$ microsomal protein.

RNA preparation Liver pieces weighing 100 to $150 \mathrm{mg}$ were taken from each fish for RNA isolation. Two pooled RNA samples were prepared from each scup treatment group, each pooled sample consisting of equally sized liver pieces from two or three fish. Liver sections that had been frozen in liquid nitrogen were powdered in liquid nitrogen and total RNA was isolated according to the guanidinium thiocyanate procedure of Chirgwin (Chirgwin et al., 1979; Clemens, 1986). Modifications were as follows: instead of two high-speed centrifugation steps to clarify the RNA solution, RNA pellets were washed once in $4 \mathrm{M} \mathrm{LiCl}$ to remove glycogen, followed by two washes in $95 \%$ ethanol to remove the salt. Total RNA was quantified on a Shimadzu UV-260 spectrophotometer, by diluting samples in water and measuring the absorption at $260 \mathrm{~nm}$ minus absorption at $300 \mathrm{~nm}$.

Northern blotting Samples of total RNA (10 $\mu \mathrm{g}$ per lane, as determined spectrally) were denatured in formamide/formaldehyde, and separated in a $1 \%$ agarose $/ 2.2 \mathrm{M}$ 
formaldehyde gel, using $20 \mathrm{mM}$ MOPS running buffer, according to standard protocols (Sambrook et al., 1989). RNA was transferred from the gel to a positively charged nylon membrane (Amersham Hybond) in 20x SSC buffer (Sambrook et al., 1989), and subsequently cross-linked to the membrane with a Fotodyne DNA transfer lamp (30 sec uv irradiation). Dried membrane was pre-hybridized for one hour at $60^{\circ} \mathrm{C}$, in a buffer composed of $6 x$ SSPE, $0.5 \%$ SDS, 10x Denhardt's solution, and $100 \mathrm{mg} / \mathrm{ml}$ calf thymus DNA. Hybridization was carried out at $42^{\circ} \mathrm{C}$ for approximately 20 hours, with a $32 \mathrm{P}$ labeled probe (see below) in a buffer composed of 5x SSPE, $0.1 \%$ SDS, $2 x$ Denhardt's solution, and $50 \%$ formamide. Probed membranes were washed for 30 minutes at $42^{\circ} \mathrm{C}$ with $2 x$ SSC, $0.1 \%$ SDS, and exposed to Kodak X-OMAT AR film for various amounts of time. Relative amount of CYP1A1 mRNA in each probe-reactive band was determined by measuring optical density (O.D.) of bands using gel plotting macros in NIH Image 1.54 software. Some reported values for CYP1A1 mRNA were normalized to total polyA mRNA in each sample (see below).

For analysis of CYP1A1 mRNA, a cDNA probe containing $75 \%$ of the scup CYP1A1 coding region was prepared. The probe hybridizes to CYP1A1 nucleotides 2591443, corresponding to amino acids 88-481 (Morrison et al., submitted). The probe was radiolabeled with a Boehringer-Manheim random-primed DNA labeling kit, using [ $\alpha-32 \mathrm{P}]$ $\mathrm{dCTP}$ (Amersham), and was cleaned with a NACS Prepac column (BRL, Inc.) according to manufacturers' instructions.

Dot blotting To determine the amount of total polyA mRNA loaded in each lane of the Northern blots, dot blots were conducted on duplicate samples using a polyT probe. A Millipore Milliblot apparatus was used, with an Amersham Hybond positively charged nylon membrane. Samples consisted of $10 \mu \mathrm{g}$ total RNA (determined spectrally), brought to a volume $50 \mu \mathrm{l}$ in TE buffer (10 mM Tris, pH 7.5, 1 mM EDTA), $30 \mu \mathrm{l} 20 \mathrm{x}$ SSC, and $20 \mu 137 \%$ formaldehyde. Samples were heated to $60^{\circ} \mathrm{C}$ for 15 minutes and then chilled on ice. Wells of the dot blot apparatus were pre-washed with 20x SSC, samples were applied (each added at 4, 2, 1, 0.5, 0.25, and $0.125 \mu \mathrm{g}$ RNA), and 20x SSC was applied to the wells again. RNA was cross-linked to the membrane using a Fotodyne DNA transfer lamp (as above). ${ }^{35}$ S-labeled polyT probes were prepared, and blots were hybridized, according to Hollander and Fornace (1990).

Total polyT bound to each dot (total polyA mRNA in each dot) was quantified by cutting the membrane into equal sized squares, each containing one dot, adding each square to a vial containing $5 \mathrm{ml}$ Scintiverse II scintillation fluid (Fisher Scientific), and counting the samples on a Beckman LS5000TD liquid scintillation counter using a wide window (0- 
670). The entire range of loadings gave a linear response (cpm polyT versus $\mu \mathrm{g}$ RNA loaded), so the $1 \mu \mathrm{g}$ loading was selected for normalizing Northern blot results.

Normalized CYP1A1 mRNA content in each pooled liver sample was calculated as follows: relative CYP1A1 mRNA O.D. / relative polyA mRNA cpm, where "relative CYP1A1 mRNA O.D." was calculated by dividing the O.D. for each Northern blot band by the O.D. of the strongest band (giving values of 0 - 1), and "relative polyA mRNA cpm" was calculated by dividing the cpm for each dot blot band ( $1 \mu \mathrm{g}$ RNA loadings) by the cpm for the dot of the uninjected fish pool.

The relative content of CYP1A1 mRNA in Northern blots was determined in two ways: (1) by normalizing to total polyA mRNA per sample, with dot blotting, and (2) by normalizing to total RNA content in each sample, determined spectrally. The two methods gave almost identical patterns of relative CYP1A1 mRNA expression among the samples.

TCB residues Content of 3,3',4,4'-tetrachlorobiphenyl was quantified in pooled samples, consisting of liver or muscle sections from all fish within a treatment group. Tissue extraction followed AOAC Method 983.21 (Helrich, 1990). Samples were mixed with sodium sulfate and pet ether, homogenized, and serially extracted with pet ether. Extracts were cleaned up on a Florisil column and concentrated under nitrogen. Extracts were analyzed for TCB by gas chromatography with electron capture detection (GC/ECD) using an HP 5890 Series II GC/ECD. Confirmation of the TCB was performed by gas chromatography with mass spectrometry (GC/MS) using an HP 5890/5970 GC/MS by monitoring molecular ions 110, 150, 184, 220, 222, 290, 292, and 294. The fragmentation pattern of each sample was compared to the TCB standard and TCB was quantified with $\mathrm{m} / \mathrm{z} 292$. Measured concentrations of TCB were corrected for extraction efficiency using the recovery of 4,4'-dibromooctafluorobiphenyl (DBOFB) which was spiked into each sample prior to extraction, and were quantified relative to the internal standard 2,4,5,6-tetrachloro-m-xylene (TCMX). Recoveries of DBOFB ranged from 60 to $96 \%$ with an average of $76 \%$. The average recovery of TCB matrix spikes was $110 \%$. The precision of duplicate analyses was within $3 \%$.

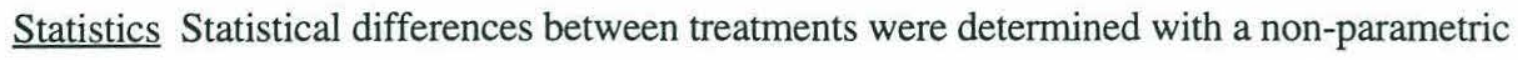
randomization test, which allowed pairwise comparisons between each of the three treatments, taking into account the entire time-course. $X_{\mathrm{ijk}}=$ measurement for fish $\mathrm{k}$, at time $\mathrm{j}$, under treatment $\mathrm{i}$. The null model is that the distribution of $\mathrm{X}_{\mathrm{ijk}}$ depends only on $\mathrm{j}$ (i.e. while there may be a time effect, there is neither a treatment effect nor an individual 
fish effect). The alternative model is that the distribution of $\mathrm{X}_{\mathrm{ijk}} \mathrm{k}$ depends on both $\mathrm{j}$ and $\mathrm{i}$ (i.e. there is both a time effect and a treatment effect). The test statistic was:

$$
\sum_{i=1}^{3} \sum_{j=1}^{n_{i}} \sum_{k=1}^{n_{i j}}\left(X_{i j k}-\bar{X}_{. j} .\right)^{2} \quad-\sum_{i=1}^{3} \sum_{j=1}^{n_{i}} \sum_{k=1}^{n_{i j}}\left(X_{i j k}-\bar{X}_{i j} .\right)^{2}
$$

where $\bar{X}_{. j}=\sum_{i=1}^{3} \sum_{k=1}^{n_{i j}} X_{i j k} / \sum_{i=1}^{3} n_{i j}$

and

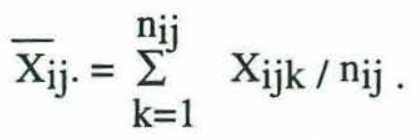

That is, $\bar{X}_{. j}$. is the sample mean for all fish at time $\mathrm{j}$ and $\overline{\mathrm{X}}_{\mathrm{ij}}$. is the sample mean for all fish under treatment $\mathrm{i}$ at time $\mathrm{j}$. The test statistic measures the improvement in the residual sum of squares by using treatment means instead of an overall mean at each time.

The distribution of the test statistic was estimated under $\mathrm{H}_{\mathrm{O}}$ by randomly permuting the treatment labels on the fish at each time point and calculating the value of the test statistic. The significance level (p-value) was estimated by the proportion of such permutations for which the value of the test statistic exceeded the observed value.

In vitro studies of CYP1A1 modification The methods of Shimada and Sato (1978) were used, with modifications, to assess covalent binding of TCB metabolites to microsomal protein. Incubations were performed at $30^{\circ} \mathrm{C}$ (fish) or $37^{\circ} \mathrm{C}$ (rats) under air in a shaking incubator. The $1 \mathrm{ml}$ assay contained $1 \mathrm{mg}$ microsomal protein from feral scup, scup treated with 2,3,7,8-tetrachlorodibenzofuran ( $3 \mu \mathrm{g} / \mathrm{kg}$; Hahn and Stegeman, 1994), or rat treated with 3-methylcholanthrene (20 mg/kg; Melancon et al., 1990), $2 \mu \mathrm{M}{ }^{14} \mathrm{C}$-labeled TCB (52.1 mCi/mmol, Pathfinder, St. Louis, MO) dissolved in acetone, $50 \mathrm{mM}$ Tris, $\mathrm{pH} 7.6$, and 0.1 mM EDTA. Analysis of the ${ }^{14} \mathrm{C}$-labeled TCB by gas chromatography revealed no hydrophobic contaminant peaks (>99\% pure); small polar contaminants were completely removed by dissolving the TCB in hexane and extracting twice with $\mathrm{NaOH}$. After a 2 minute pre-incubation, reactions were initiated with $1 \mathrm{mM} \mathrm{NADPH}$ and run for 60 minutes. Assays were stopped with $1.5 \mathrm{ml}$ of $10 \%$ ice-cold trichloroacetic acid (TCA) followed by centrifugation at $5000 \mathrm{~g}$ for 30 minutes, to precipitate protein. Pellets were washed several more times, to remove parent TCB and TCB products which were not covalently linked to protein. The 1.5-ml washes were as follows: 1x 10\% TCA, 2x 80\% methanol, $2 x$ methanol: diethyl ether (1:1), 2x n-hexane, $2 x 80 \%$ methanol. The final wash was determined to have ${ }^{14} \mathrm{C}$ levels near background. Protein-bound metabolites were quantified by dissolving the final pellet in $1 \mathrm{~N} \mathrm{NaOH}$ and measuring ${ }^{14} \mathrm{C}$ in the pellet with 
liquid scintillation counting on a Beckman LS5000TD counter. Metabolic activity was determined by subtracting the pellet radioactivity of control incubations, in which no NADPH cofactor was added.

Hepatic microsomes from both feral scup and scup treated with 3methylcholanthrene $\left(20 \mathrm{mg} / \mathrm{kg}\right.$, chapter 5) were incubated at $30^{\circ} \mathrm{C}$ for 30 minutes in a Tris buffer (as above), with or without TCB (1.5 $\mathrm{MM}$ in DMSO), and with or without NADPH cofactor $(1 \mathrm{mg} / \mathrm{ml})$, and analyzed spectrophotometrically for cytochrome P450 content as above. Microsomal protein content in the incubations was $0.5 \mathrm{mg} / \mathrm{ml}$. Additional incubations were conducted in the presence of sodium azide ( $1 \mathrm{mM})$.


incubated at $1 \mathrm{mg} / \mathrm{ml}$ in $50 \mathrm{mM}$ Tris, $\mathrm{pH} \mathrm{7.6}$, and $0.1 \mathrm{mM}$ EDTA, with the addition of either 3,3',4,4'-TCB (1.5 $\mu \mathrm{M}$ in acetone), 2,2',5,5'-TCB (1.5 $\mu \mathrm{M}$ in acetone), or acetone alone, and in the presence or absence of NADPH $(1.5 \mathrm{mg} / \mathrm{ml})$. Incubations were performed at $30^{\circ} \mathrm{C}$ under air in a shaking incubator. At 15 minutes, 30 minutes, and 60 minutes, aliquots of each incubation were removed for measurement of EROD activity. To the aliquot of incubation, EROD buffer was added (100 mM Tris, $100 \mathrm{mM} \mathrm{NaCl}, \mathrm{pH} 8)$ to a final concentration of $2 \mu \mathrm{M}$ 7-ethoxyresorufin (7-ER) substrate. NADPH was also added, to those microsomal incubations that had been conducted in the absence of NADPH. The rate of 7-ER O-deethylation was monitored spectrally for 3 minutes, as previously described (Klotz et al., 1984). The entire experiment was conducted twice, and each time, each incubation was performed in triplicate. An identical set of incubations was conducted, and analyzed for NADPH cytochrome c (P450) reductase activity at each time point, assayed by monitoring absorbance at $550 \mathrm{~nm}$ as previously described (Stegeman et al., 1979).

In a similar set of studies, hepatic microsomes from feral scup were incubated with or without NADPH, in the presence of either $1.5 \mu \mathrm{M}$ TCB, $1.5 \mu \mathrm{M}$ benzo[a]pyrene (BaP), or no substrate. Some incubations included exogenous catalase (bovine liver, Sigma, 800 units $/ \mathrm{ml})$, and some included sodium azide $(1 \mathrm{mM})$ to inhibit endogenous catalase. Aliquots of the incubations were removed at times $0,10,20$, and 30 minutes, for fluorometric determination of EROD activity, as described previously (Hahn et al., 1993). All experiments were conducted twice, and each time, each incubation was performed in duplicate. 


\section{Results}

Hepatic monooxygenase components

Content of total P450 in hepatic microsomes was strongly induced by $0.1 \mathrm{mg} / \mathrm{kg} \mathrm{TCB}$ on days 2 to 16 (Fig 1A). Maximal induction was attained by day 5 (3-fold increase over corn oil controls), and was sustained until the end of the time-course. Total P450 was not significantly induced by $5.0 \mathrm{mg} / \mathrm{kg}$ TCB on days 2 to 12 . However, on day 16 , of the four fish treated with $5.0 \mathrm{mg} / \mathrm{kg}$, two had very low P450 levels (similar to all other fish treated with $5.0 \mathrm{mg} / \mathrm{kg} \mathrm{TCB}$, and similar to controls), while two had strongly induced P450 (similar to levels in fish treated with $0.1 \mathrm{mg} / \mathrm{kg} \mathrm{TCB}$ ).

The specific content of cytochrome b5 was increased approximately two-fold by TCB treatment (days 5,16 ). Unlike P450, the increase in b5 content was similar at the two TCB doses (Fig 1B).

Hepatic microsomes were immunoblotted with antibodies raised against the three major P450 forms expressed in scup liver: P450E (a CYP1A1 homologue; Park et al., 1986), P450B (a probable CYP2B homologue; Stegeman et al., 1990a), and P450A (a possible CYP3A homologue; Celander et al., 1994) (Fig 2). CYP1A1 content was strongly induced by $0.1 \mathrm{mg} / \mathrm{kg} \mathrm{TCB}$, reaching a peak on day 5 (12-fold induction over control) and showing signs of subsequent decline (Fig 2A). In contrast, CYP1A1 was only weakly, but significantly, induced in fish treated with $5.0 \mathrm{mg} / \mathrm{kg} \mathrm{TCB}$, also reaching a peak on day 5 (4-fold induction over control). Of the four fish treated with $5.0 \mathrm{mg} / \mathrm{kg} \mathrm{TCB}$ and sampled on day 16, the two that had elevated levels of total P450 also had two-fold more CYP1A1 than did the two fish with low total P450 content.

The molar increases in CYP1A1 accounted for approximately $85 \%$ of the molar increases in total P450, over control levels (Table 1). Thus both the strong induction in total P450 at $0.1 \mathrm{mg} / \mathrm{kg}$, and the "suppressed" induction of total P450 at $5.0 \mathrm{mg} / \mathrm{kg}$, were primarily due to differences in CYP1A1 content.

Differences between groups were less clear for hepatic P450B (Fig 2B) and P450A (Fig 2C). The content of both proteins showed temporal patterns resembling the changes in content of CYP1A1. Thus, both P450B and P450A showed an initial increase in enzyme content (days 2,5 ) followed by a decline in the fish treated with $0.1 \mathrm{mg} / \mathrm{kg}$, and enzyme content was closer to control values in the fish treated with $5.0 \mathrm{mg} / \mathrm{kg}$ (day 5). However, content of both enzymes varied widely within each treatment group (large standard deviations about the means), suggesting regulation by factors apart from TCB treatment. Furthermore, the differences in mean enzyme content between treated and control groups were far smaller for P450B and P450A than for CYP1A1. 

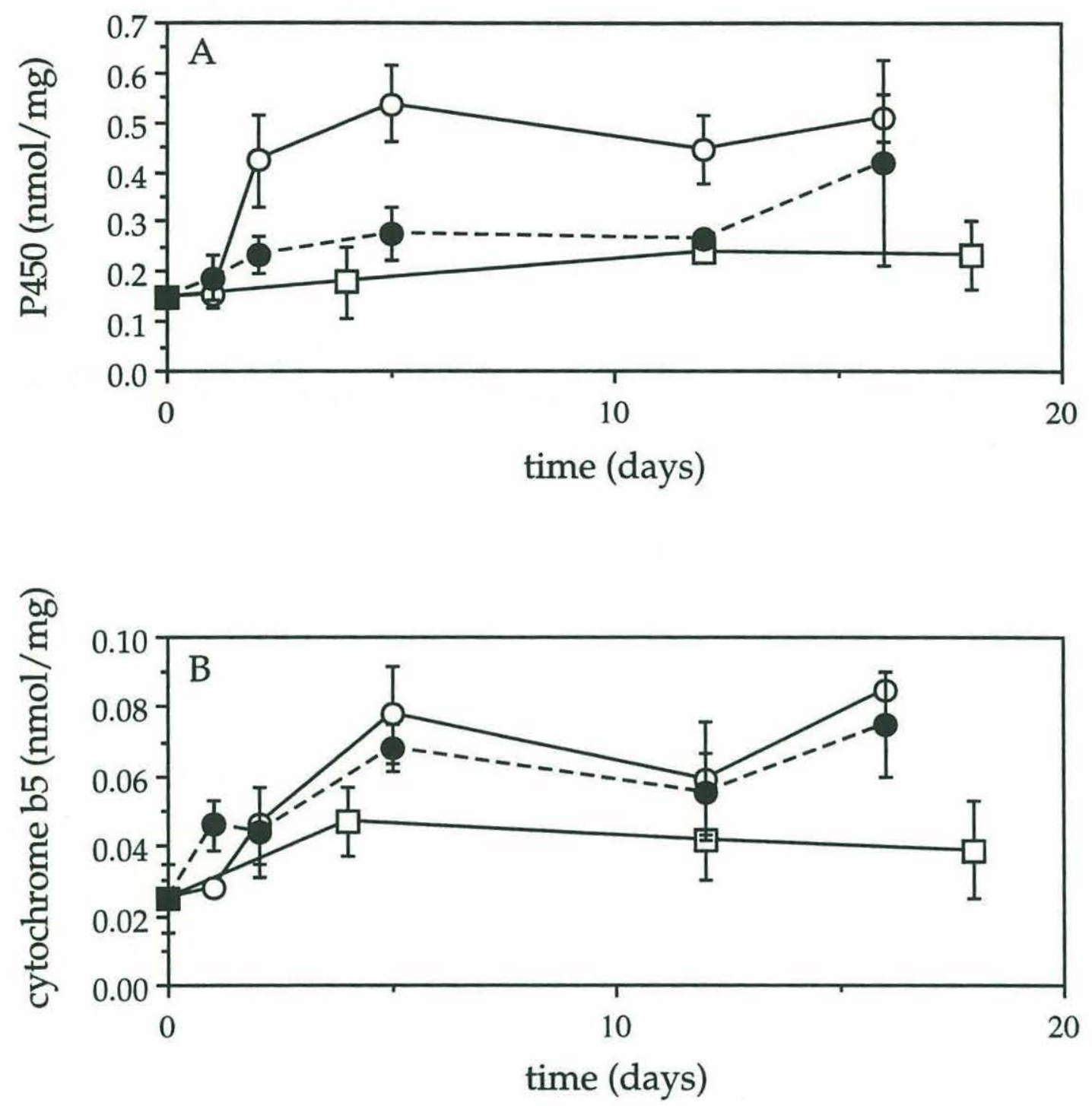

Figure 1. Hepatic microsomal content of total cytochrome P450 (A) and cytochrome b5 (B), determined spectrally as described in Methods. $\mathrm{X}$-axis is number of days subsequent to injection, $\mathrm{y}$-axis is enzyme content in $\mathrm{nmol}$ enzyme/ $\mathrm{mg}$ microsomal protein. Each point represents the mean and standard deviation (n-1) of measurements on four or five fish per treatment group. Plot symbols are: square, corn oil control, open circle, $0.1 \mathrm{mg} / \mathrm{kg} \mathrm{TCB}$, closed circle, $5.0 \mathrm{mg} / \mathrm{kg}$ TCB . Cytochrome $\mathrm{P} 450$ content is significantly greater in the 0.1 $\mathrm{mg} / \mathrm{kg}$ treatment group than in the other two groups at $\mathrm{p} \leq 0.0006$. Cytochrome b5 content is significantly greater in both TCB treatment groups than in controls at $\mathrm{p} \leq 0.0002$. 
TABLE 1

Induction of Total Hepatic Cytochrome P450 due to CYP1A1

\begin{tabular}{llll}
\hline & control & $0.1 \mathrm{mg} / \mathrm{kg}$ TCB & $5.0 \mathrm{mg} / \mathrm{kg}$ TCB \\
\hline $\begin{array}{l}\text { Total P450 } \\
(\mathrm{pmol} / \mathrm{mg})\end{array}$ & $178 \pm 71$ & $538 \pm 75$ & $278 \pm 53$ \\
$\begin{array}{l}\text { P450 Induction } \\
\text { over Control } b\end{array}$ & -- & 360 & 100 \\
$\begin{array}{l}\text { CYP1A1 } \\
\text { (pmol/mg) } a\end{array}$ & $27 \pm 0.1$ & $332 \pm 33$ & $111 \pm 51$ \\
$\begin{array}{l}\text { CYP1A1 Induction } \\
\text { over Control } b\end{array}$ & -- & 305 & 84 \\
\%P450 Induction & -- & $85 \%$ & $84 \%$ \\
due to CYP1A1 & & & \\
\hline
\end{tabular}

$a$ Values represent mean \pm SD (n-1) for five fish (TCB-treated) or four fish (control).

Animals were sampled on day 5 (TCB) or day 4 (control) after treatment.

$b$ Determined by subtracting enzyme content in control fish from enzyme content in TCBtreated fish. Units are $\mathrm{pmol} / \mathrm{mg}$. 
Figure 2. Hepatic microsomal content of CYP1A1 (A), P450B, the putative CYP2B (B), and P450A, the putative CYP3A (C), determined by immunoblotting as described in Methods. X-axis is number of days subsequent to injection, $\mathrm{y}$-axis is enzyme content in $\mathrm{pmol} / \mathrm{mg}$ microsomal protein (A) and in relative optical density/ $\mu \mathrm{g}$ (A, B, C). Each point represents the mean and standard deviation (n-1) of measurements on four or five fish per treatment group. Plot symbols are: square, corn oil control, open circle, $0.1 \mathrm{mg} / \mathrm{kg}$ TCB, closed circle, $5.0 \mathrm{mg} / \mathrm{kg}$ TCB. CYP1A1 content is significantly different between each of the three treatment groups ( $\mathrm{p} \leq 0.0003$ ). P450B content is significantly different between control and $5.0 \mathrm{mg} / \mathrm{kg}$, and between $0.1 \mathrm{mg} / \mathrm{kg}$ and $5.0 \mathrm{mg} / \mathrm{kg}(\mathrm{p} \leq 0.008)$, but is not different between $0.1 \mathrm{mg} / \mathrm{kg}$ and control. P450A content is significantly different between control and $0.1 \mathrm{mg} / \mathrm{kg}$ only $(\mathrm{p}=0.05)$. 

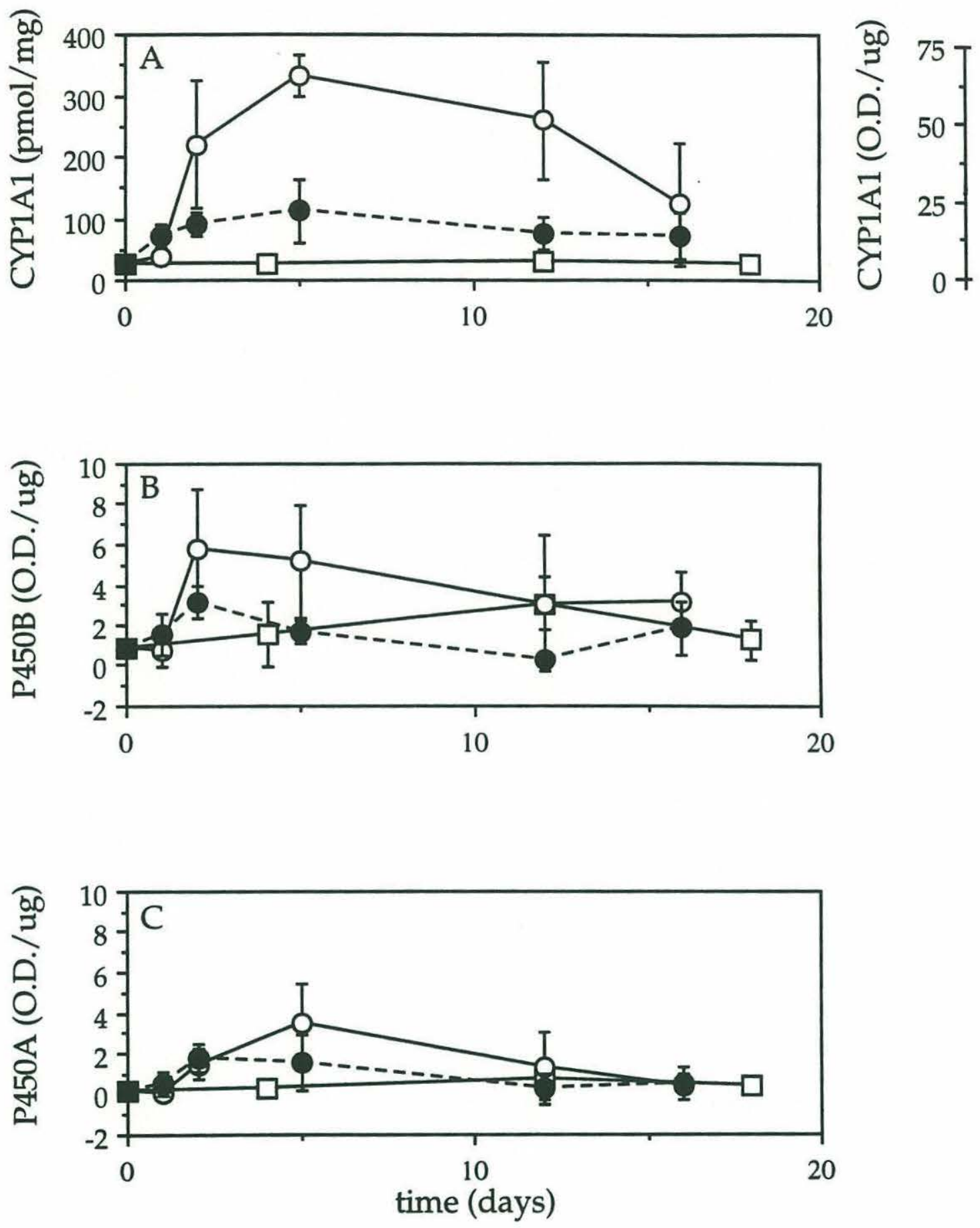
Microsomal enzyme activities

Several enzyme activities were measured in hepatic microsomes (Figs 3 and 4), to determine whether suppression of enzyme content by TCB was specific for CYP1A1. Ethoxyresorufin O-deethylase (EROD) activity, catalyzed principally by CYP1A1 in fish (Klotz et al., 1983), was strongly induced by $0.1 \mathrm{mg} / \mathrm{kg}$ TCB, reaching maximal levels by day 5 that were sustained until the end of the experiment (Fig 3A). The 15-fold induction in EROD activity on day 5 mirrored the 12-fold induction in CYP1A1 content on day 5. In fish treated with $5.0 \mathrm{mg} / \mathrm{kg} \mathrm{TCB}$, there was no induction of EROD activity above control levels from days 1 through 12, even though there was a significant induction in CYP1A1 protein content in these groups. Suppressed EROD rates at the high dose are consistent with competitive inhibition of EROD activity by residual TCB in microsomes (e.g. Gooch et al., 1989), and indicate that TCB is retained in the CYP1A1 active site. As with total P450 and CYP1A1, an exception occurred on day 16, at which time the two fish treated with $5.0 \mathrm{mg} / \mathrm{kg}$ TCB that had elevated CYP1A1 content also had strongly induced EROD activity, while EROD rates remained low in the other two fish.

Pentoxyresorufin O-depentylase (PROD) activity displayed a profile almost identical to that of hepatic EROD activity (Fig 3B). Although PROD is largely catalyzed by CYP2B enzymes in mammals (Burke et al., 1985), the catalyst(s) for this activity in teleosts have not been identified. The pattern seen here suggests that CYP1A1 contributes to this activity in scup that have been treated with a CYP1A inducer.

NADPH cytochrome c (P450) reductase activity was induced approximately $50 \%$ in treated scup (days 5 - 16), and levels did not differ between the two TCB doses (Fig 4A). Hepatic microsomal UGT activity with $p$-nitrophenol was induced approximately two-fold in fish treated with TCB and sampled on day 5 (Fig 4B). Rates were statistically equivalent in fish treated with the two TCB doses.

\section{Hepatic CYP1A1 mRNA expression}

Content of CYP1A1 mRNA was quantified in two pooled samples from each treatment group, each sample consisting of liver tissue from 2 or 3 fish. Northern blots of total RNA were performed with a cDNA probe containing $75 \%$ of the scup CYP1A1 coding region, which hybridized to a single band in each sample (Fig 5). The relative content of CYP1A1 mRNA was similar in fish administered the two TCB doses, with maximum expression on days 1,2 , and 5 , which diminished by day 12 (Fig 6). In fish treated with $0.1 \mathrm{mg} / \mathrm{kg}$ $\mathrm{TCB}, \mathrm{mRNA}$ content continued to decline by day 16 , whereas fish receiving the higher dose seemed to express a second peak in CYP1A1 mRNA content on day 16. The mRNA 



Figure 3. Hepatic microsomal EROD activity (A) and PROD activity (B), determined fluorometrically as described in Methods. X-axis is number of days subsequent to injection, $\mathrm{y}$-axis is rate of enzyme activity in $\mathrm{pmol} / \mathrm{min} / \mathrm{mg}$ microsomal protein. Each point represents the mean and standard deviation (n-1) of measurements on four or five fish per treatment group. Plot symbols are: square, corn oil control, open circle, $0.1 \mathrm{mg} / \mathrm{kg}$ TCB, closed circle, $5.0 \mathrm{mg} / \mathrm{kg}$ TCB. For both activities, rates in the $0.1 \mathrm{mg} / \mathrm{kg}$ group were significantly greater than controls at $\mathrm{p} \leq 0.0001$, and rates in the $0.1 \mathrm{mg} / \mathrm{kg}$ group were significantly greater than the $5.0 \mathrm{mg} / \mathrm{kg}$ group at $\mathrm{p} \leq 0.005$. 



Figure 4. Hepatic microsomal NADPH reductase activity (A), and UGT activity (B), measured spectrally as described in Methods. Y-axes are rates of enzyme activity in $\mathrm{nmol} /$ $\mathrm{min} / \mathrm{mg}$ microsomal protein. (A) X-axis is number of days subsequent to injection. Each point represents the mean and standard deviation (n-1) of measurements on four or five fish per treatment group. Plot symbols are: square, corn oil control, open circle, $0.1 \mathrm{mg} / \mathrm{kg}$ TCB, closed circle, $5.0 \mathrm{mg} / \mathrm{kg}$ TCB. Rate of activity is significantly greater in both TCB treatment groups than in controls at $p \leq 0.004$. (B) Bars represent means and standard deviations (n-1) of measurements on 4 samples each. Assays were performed on animals sampled on day 4 (control) or 5 (TCB treated). Rates in the two TCB treatment groups were statistically equivalent, and rates in each were statistically elevated over control fish ( $p$ $\leq 0.01$ ), based on a one-way ANOVA. 

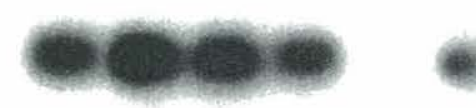

\section{$\begin{array}{lllll}1 & 2 & 3 & 4 & 5\end{array}$}

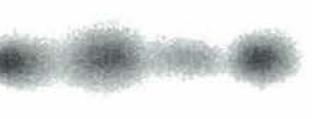

$\begin{array}{llllllllll}7 & 8 & 9 & 10 & 11 & 12 & 13 & 14 & 15 & 16\end{array}$

Figure 5. Northern blot of hepatic CYP1A1 mRNA in pooled scup samples. Each sample consists of pooled liver tissue from 2 or 3 fish. Samples of total RNA (10 $\mu \mathrm{g}$ per lane) were separated on an agarose gel, transferred to nylon membrane, and probed with a $32 \mathrm{P}$ labelled cDNA probe containing $75 \%$ of the scup CYP1A1 coding region, as described in Methods. Samples in the lanes are as follows: (1) time zero (uninjected), (2) day 1, 0.1 $\mathrm{mg} / \mathrm{kg}$ (3) day $1,5.0 \mathrm{mg} / \mathrm{kg}$, (4) day $2,0.1 \mathrm{mg} / \mathrm{kg}$, (5) day $2,5.0 \mathrm{mg} / \mathrm{kg}$, (6) day 4 , corn oil, (7) day $5,0.1 \mathrm{mg} / \mathrm{kg}$, (8) day $5,5.0 \mathrm{mg} / \mathrm{kg}$, (9) day $12,0.1 \mathrm{mg} / \mathrm{kg}$, (10) day $12,5.0$ $\mathrm{mg} / \mathrm{kg}$, (11) day 12 , corn oil, (12) day $16,0.1 \mathrm{mg} / \mathrm{kg}$, (13) day $16,5.0 \mathrm{mg} / \mathrm{kg}$ with induced P450 content, (14) day 16, $5.0 \mathrm{mg} / \mathrm{kg}$ with uninduced P450 content, (15) day 18 , corn oil, (16) day $34,0.1 \mathrm{mg} / \mathrm{kg}$. 


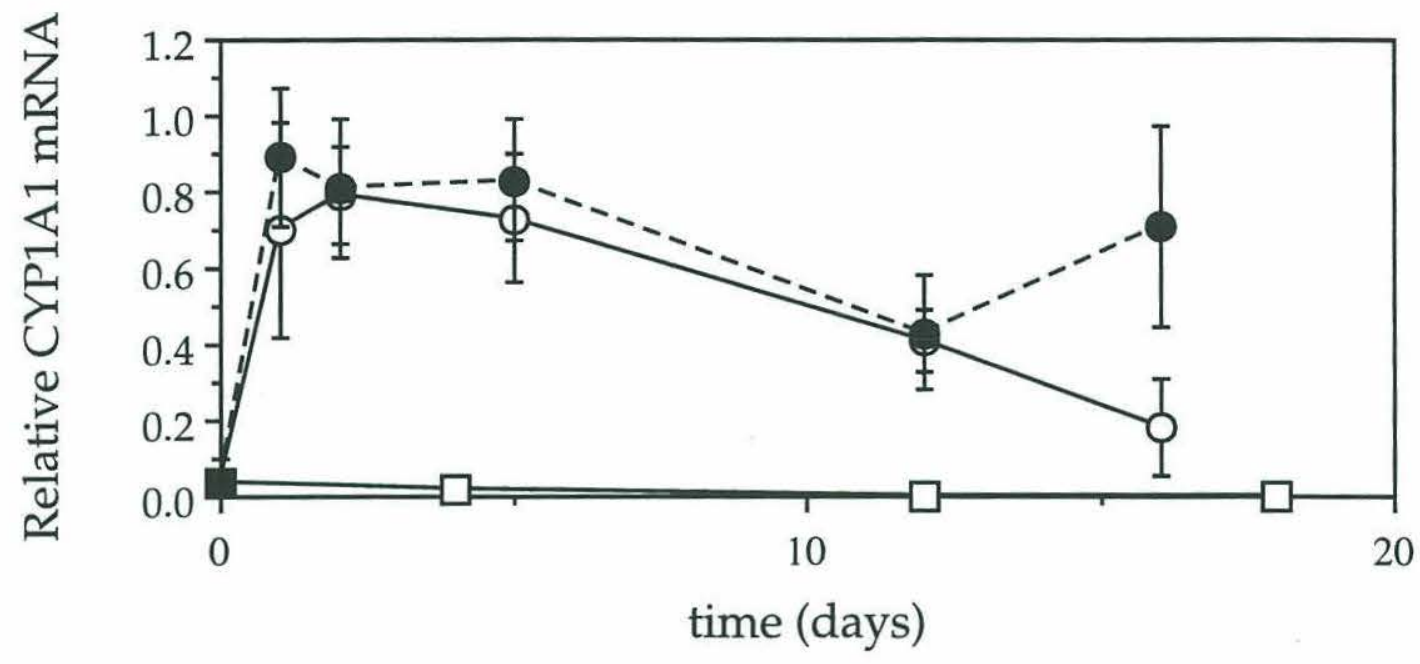

Figure 6. Hepatic CYP1A1 mRNA content, determined as described in Fig 6. X-axis is number of days subsequent to injection, $y$-axis is relative content of CYP1A1 mRNA, determined by measuring the optical density of Northern blot bands and normalizing to total RNA content in each sample, determined spectrally. Each point represents the mean and standard deviation (n-1) of measurements on two samples per treatment group, each sample consisting of liver sections from 2 or 3 fish. Plot symbols are: square, corn oil control, open circle, $0.1 \mathrm{mg} / \mathrm{kg} \mathrm{TCB}$, closed circle, $5.0 \mathrm{mg} / \mathrm{kg}$ TCB. Content of total polyA mRNA was measured in some samples by dot blotting, and CYP1A1 mRNA content determined by normalizing to total polyA rather than to total RNA. Both methods of normalizing gave very similar results for relative CYP1A1 mRNA content among the samples. 


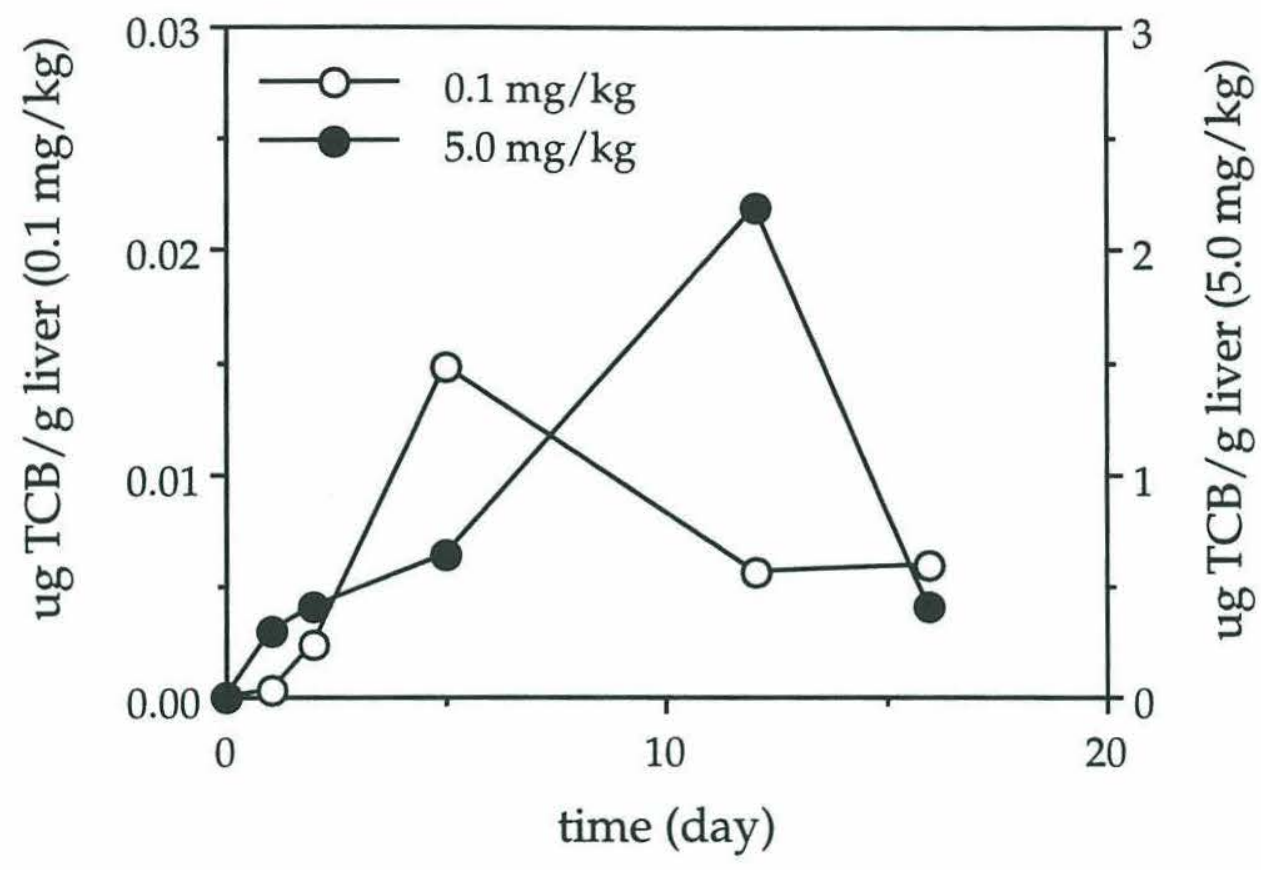

Figure 7. GC/MS analysis of TCB content in pooled liver samples from TCB-treated fish. $\mathrm{X}$-axis is number of days subsequent to injection. Y-axis is TCB concentration in $\mu \mathrm{g} \mathrm{TCB} /$ $\mathrm{g}$ liver dry weight; left axis is concentration in fish treated with $0.1 \mathrm{mg} / \mathrm{kg}$, right axis is TCB concentration in fish treated with $5.0 \mathrm{mg} / \mathrm{kg}$ (note the difference in scale). Each point is a measurement on a single sample, consisting of pooled tissue sections from all 4 or 5 fish in each treatment group. Plot symbols are: open circle, $0.1 \mathrm{mg} / \mathrm{kg} \mathrm{TCB}$, closed circle, $5.0 \mathrm{mg} / \mathrm{kg}$ TCB. 
content in the latter group was particularly high in the livers of the two fish that had elevated P450 content on day 16.

\section{TCB residues}

TCB content was measured in pooled liver samples from each treatment group (Fig 7).

The average dry weight in liver samples was determined to be $25 \%$ of the wet weight; dry weights are presented here. In fish treated with $0.1 \mathrm{mg} / \mathrm{kg} \mathrm{TCB}$, the TCB content in liver reached a maximum concentration of $0.015 \mu \mathrm{g} / \mathrm{g}$ liver on day 5 . In fish treated with 5.0 $\mathrm{mg} / \mathrm{kg}$ TCB, the TCB content in liver did not reach a maximum until day 12 , at which time the concentration was $2.2 \mu \mathrm{g} \mathrm{TCB} / \mathrm{g}$ liver. At both doses, hepatic TCB content on day 16 was substantially lower than the peak concentration. Analysis of bile residues indicates that this depuration was due to both metabolism and to loss of parent compound (White et al., in preparation).

\section{CYP1A1 modification in vitro}

TCB was metabolized to reactive forms in vitro, which bound covalently to microsomal proteins, at a very slight rate (Table 2). The rate of TCB activation in hepatic microsomes from feral scup was $1.5 \mathrm{pmol}$ metabolites covalently bound to total protein / hour incubation / $\mathrm{mg}$ microsomal protein added to incubation. The rate was similar in hepatic microsomes from scup treated with a CYP1A inducer (2,3,7,8-tetrachlorodibenzofuran). In contrast, hepatic microsomes from rat treated with a CYP1A inducer (3methylcholanthrene) produced covalently-bound TCB metabolites at a rate of 99 $\mathrm{pmol} / \mathrm{min} / \mathrm{mg}$ in our hands.

Incubation of hepatic microsomes from feral scup with TCB, or with TCB plus $\mathrm{NADPH}$, had no demonstrable effect on spectrally detectable cytochrome P450 (not shown). However, the pool of feral scup microsomes used had an approximate CYP1A1 content equal to only $15 \%$ of the total P450. Therefore, similar incubations were conducted using a microsomal sample from a 3-methylcholanthrene-treated scup, which had an approximate CYP1A1 content equal to $45 \%$ of the total P450. Incubation of hepatic microsomes for 30 minutes with TCB plus NADPH caused the conversion of approximately $40-50 \%$ of the cytochrome P450 to the denatured form, cytochrome P420 (Fig 8; Table 3). This conversion appeared quantitative, with P420 accounting for almost all of the P450 that was lost, assuming that extinction coefficients in scup are similar to those in rat (Omura and Sato, 1964). No P450 loss or P420 formation was seen in incubations containing TCB alone or NADPH alone (not shown), indicating a requirement for both substrate binding and electron transport in the enzyme denaturation. 
TABLE 2

Metabolites of 3,3',4,4'-TCB Covalently Bound to Microsomal Protein

\begin{tabular}{llll}
\hline microsomes $a$ & reaction & $1{ }^{\mathrm{C}-\mathrm{TCB}} b$ & $\mathrm{pmol} / \mathrm{hr} / \mathrm{mg} d$ \\
\hline Feral scup & $+\mathrm{NADPH}$ & 269.0 & 1.5 \\
& - NADPH & $101.7 \mathrm{c}$ & \\
\cline { 2 - 4 } TCDF scup & $+\mathrm{NADPH}$ & 154.8 & 1.8 \\
& $-\mathrm{NADPH}$ & 53.5 & \\
\cline { 2 - 4 } MC rat & $+\mathrm{NADPH}$ & 5790.8 & 99.3 \\
& - NADPH & 49.3 & \\
\hline
\end{tabular}

$a$ Microsomes were incubated with ${ }^{14} \mathrm{C}-\mathrm{TCB}+/$ - NADPH for 60 minutes, followed by precipitation and solvent-washing, to determine amount of TCB covalently bound to microsomal protein. Samples were from feral scup (killed immediately upon capture), depurated scup treated with 2,3,7,8-tetrachlorodibenzofuran (TCDF, $3 \mu \mathrm{g} / \mathrm{kg}$ ), or rat treated with 3-methylcholanthrene (MC, $20 \mathrm{mg} / \mathrm{kg}$ ). Rates of EROD activity were similar in TCDF scup and MC rat, suggesting a comparable degree of CYP1A1 induction.

$b$ Disintegrations per minute from ${ }^{14} \mathrm{C}$-TCB, measured in washed microsomal pellet as described in Methods. Each value is a mean of duplicate assays.

$c$ Higher background radioactivity in feral scup is due to a different batch of ${ }^{14} \mathrm{C}$-TCB substrate used.

$d$ Rates are expressed as pmol metabolites binding to total microsomal protein / hour incubation / $\mathrm{mg}$ microsomal protein added to incubation. Rates were calculated by subtracting the - NADPH blanks from the + NADPH values, then dividing by specific activity of the substrate. 


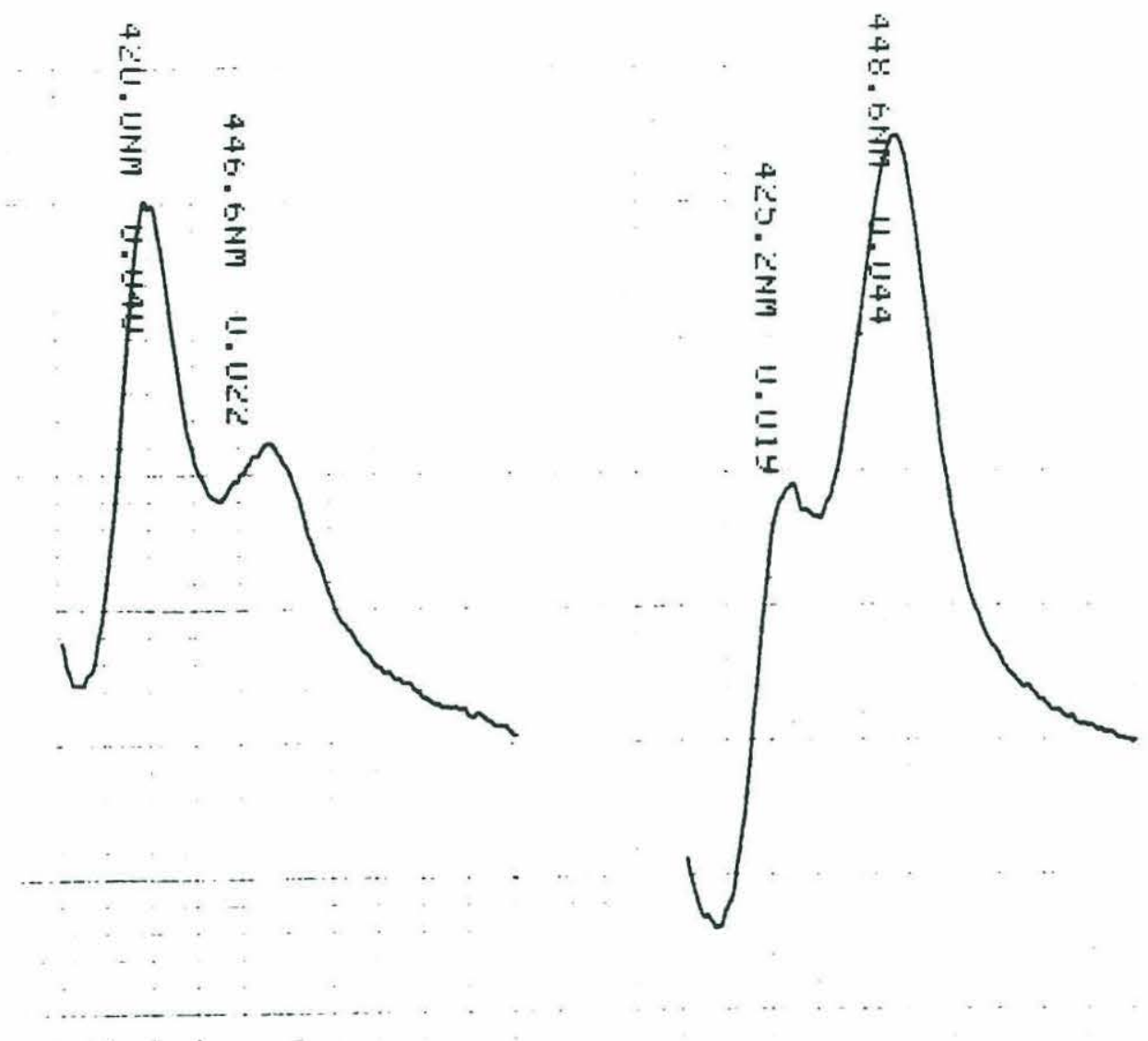

Figure 8. Spectrophotometric analysis of cytochrome P450 in hepatic microsomes from 3methylcholanthrene-treated scup. Microsomes were incubated for 30 minutes at $30^{\circ} \mathrm{C}$, either without additions (right), or with the addition of both TCB and NADPH (left), and P450 content was analyzed by measuring the dithionite difference spectrum on CO-treated samples. Cytochrome P450 absorbs at approximately $446-449 \mathrm{~nm}$, the shoulder at $425 \mathrm{~nm}$ (right) is a contaminating chromophore in this microsomal sample, and the peak at $420 \mathrm{~nm}$ (left) is due to the cytochrome P450 degradation product cytochrome P420. 


\section{TABLE 3}

Cytochrome P450 Content and EROD Activity in Microsomal Incubations at 30 Minutes

\begin{tabular}{lll}
\hline incubation & $\mathrm{P} 450 a$ & EROD \\
\hline no additions & $100 \%$ & $100 \%$ \\
NADPH & $100 \%$ & $100 \%, 100 \%$ \\
TCB & $100 \%$ & $71 \%, 75 \% c$ \\
TCB + NADPH & $50 \%, 58 \%$ & $30 \%, 31 \%$ \\
\hline azide + NADPH & $47 \%, 55 \%$ & $65 \%, 72 \%$ \\
azide + TCB & $74 \%, 89 \%$ & $40 \% c$ \\
azide + TCB + NADPH & $18 \%, 21 \%$ & $1 \%, 2 \%$ \\
\hline
\end{tabular}

Microsomes were incubated at $30^{\circ} \mathrm{C}$ for 30 minutes with the specified additions. Individual measurements from duplicate experiments are reported, as a percentage of the enzyme content or activity in control incubations (containing no additions).

a Cytochrome P450 was analyzed spectrophotometrically, in a hepatic microsomal sample from a 3-methylcholanthrene-treated scup (20 mg/kg) (chapter 5).

$b$ EROD activity was analyzed fluorometrically, in a pooled microsomal sample from livers of several feral scup. Incubations with azide addition alone had 100\% EROD activity.

$c$ Strong inhibition of EROD activity without a comparable P450 loss, in the samples that were pre-incubated with TCB and without NADPH, is presumably due to competitive inhibition. 
Hepatic microsomes from feral scup were similarly incubated with or without TCB and with or without NADPH cofactor, and analyzed for CYP1A1 content by immunoblot. None of the incubation conditions altered the quantity of immunodetectable CYP1A1, as measured with MAb 1-12-3 (not shown). This result indicates that the native (P450) and denatured (P420) forms of the enzyme have the same electrophoretic molecular weight, and suggests that actual degradation of the enzyme does not occur during a one hour incubation in vitro.

\section{CYP1A1 inactivation in vitro}

Hepatic microsomes from feral scup were similarly pre-incubated with or without TCB, and with or without NADPH cofactor, and aliquots removed for measurement of EROD activity over the course of one hour (Fig 9A). Rates of EROD activity were unchanged following pre-incubation of microsomes with NADPH alone. TCB addition alone reduced microsomal EROD activity by $50 \%$ at time zero, and activity remained the same throughout the one hour incubation. This loss, which was neither time-dependent nor cofactordependent, was presumably due to reversible inhibition of EROD activity by parent TCB (e.g. Gooch et al., 1989). Pre-incubation of microsomes with both TCB and NADPH under conditions supporting TCB metabolism (chapter 5) caused a progressive drop in EROD activity with time (Fig 9A), below that due to TCB inhibition alone, indicating substrate-dependent enzyme inactivation.

In a parallel experiment, microsomes were pre-incubated with TCB and/or $\mathrm{NADPH}$, and assayed for NADPH cytochrome c (P450) reductase activity over one hour (Fig 9B). There was no effect on reductase activity, suggesting that TCB interacts directly with the P450 and not with the reductase to cause the inactivation. In a second parallel experiment, microsomes were pre-incubated with 2,2',5,5'-TCB and/or NADPH, and assayed for EROD activity over one hour (Fig 9C). 2,2',5,5'-TCB is metabolized in scup hepatic microsomes but is not metabolized by scup CYP1A1 (chapter 5). EROD activity was unchanged by any of the incubation conditions with this ortho-substituted congener.

Similar incubations were conducted in the presence of exogenous catalase, an $\mathrm{H}_{2} \mathrm{O}_{2}$ scavenger. Catalase addition ( 800 units $/ \mathrm{ml}$ ) did not prevent the CYP1A1 inactivation occurring in microsomes with 3,3',4,4'-TCB and NADPH. Profiles were similar to those in Fig 9A (not shown).

Microsomal incubations were similarly conducted, with the addition of sodium azide. Azide is known to inhibit catalase activity, thus allowing the accumulation of any $\mathrm{H}_{2} \mathrm{O}_{2}$ that is produced in the microsomes. Azide has also been found to be converted to azidyl radical in the presence of hydroxyl radical (Reinke et al., 1994), which likely 
contributes to its mechanism of action. In the absence of substrate, pre-incubation with azide and cofactor caused a slight (28-35\%) inactivation of microsomal CYP1A1 by 30 minutes (Fig 10A). In the presence of TCB substrate, however, incubation with azide and cofactor caused a 97\% loss of CYP1A1 catalytic capacity within 10 minutes (Fig 10B), exceeding the loss seen in incubations containing TCB and cofactor alone. Addition of azide without exogenous NADPH caused no time-dependent changes in microsomal EROD activity under any of the incubation conditions (data not shown). Data for 30 minute incubations are summarized in Table 3.

Strong inactivation was not seen in the presence of azide plus $\mathrm{BaP}$, a rapidly metabolized substrate compared to TCB (chapter 5). Addition of $\mathrm{BaP}$ alone to microsomes caused a $70 \%$ inhibition of microsomal EROD activity at time zero (Fig 10C vs. 10A). This inhibition was largely released by 10 minutes in the presence of NADPH (Fig 10C). Prior work (Stegeman et al., 1981) indicates that $100 \%$ of the $\mathrm{BaP}$ added at the concentration here should have been metabolized by feral scup microsomes within 10 minutes. Azide addition caused slight inactivation of CYP1A1 during microsomal BaP metabolism: activity was decreased by $29 \%$ in the first 10 minutes of incubation, as compared to incubations with $\mathrm{BaP}$ and NADPH alone (Fig 10C). After a 30 minute incubation in the presence of azide, cofactor, and $\mathrm{BaP}$ (and $\mathrm{BaP}$ metabolites), activity was decreased by $42 \%$, as compared to incubations with BaP and NADPH alone (Fig 10C), a similar loss to that seen in incubations without substrate (Fig 10A). Thus the rapid and complete inactivation seen with TCB does not occur in the presence of substrates in general. Furthermore, the finding that azide enhances inactivation suggests that an increased production of reactive oxygen species occurs in microsomes in the presence of TCB and cofactor (see Discussion).

Analysis of spectral cytochrome P450 content in azide-containing incubations demonstrated that azide plus cofactor caused the conversion of P450 to P420, similar to that shown in Fig 8. However, the P450 loss was greatly enhanced in the presence of azide, cofactor, and TCB (Table 3), again suggesting enzyme oxidation during the process of substrate binding and electron transport. The degree of P450 loss in the presence of azide, NADPH, and TCB (approximately $80 \%$ ) indicates that inactivation was not specific for CYP1A1 under this condition. 
Figure 9. Enzyme inactivation by TCB in vitro. Hepatic microsomes from feral scup were incubated + or - TCB $(1.5 \mu \mathrm{M})$, and + or - NADPH cofactor, and aliquots of the incubations were removed at $0,15,30$, and 60 minutes for spectrophotometric measurement of residual enzyme activity in microsomes. (A) Incubations with 3,3',4,4'TCB, assayed for EROD activity. (B) Incubations with 3,3',4,4'-TCB, assayed for NADPH cytochrome c (P450) reductase activity. (C) Incubations with 2,2',5,5'-TCB, assayed for EROD activity. $\mathrm{X}$-axis is the length of time of microsomal incubations, $\mathrm{y}$-axis is the rate of catalytic activity in the incubations at given time points. Each point represents the mean and standard deviation (n-1) of catalytic activity in 6 assays (triplicate samples in two separate experiments). 


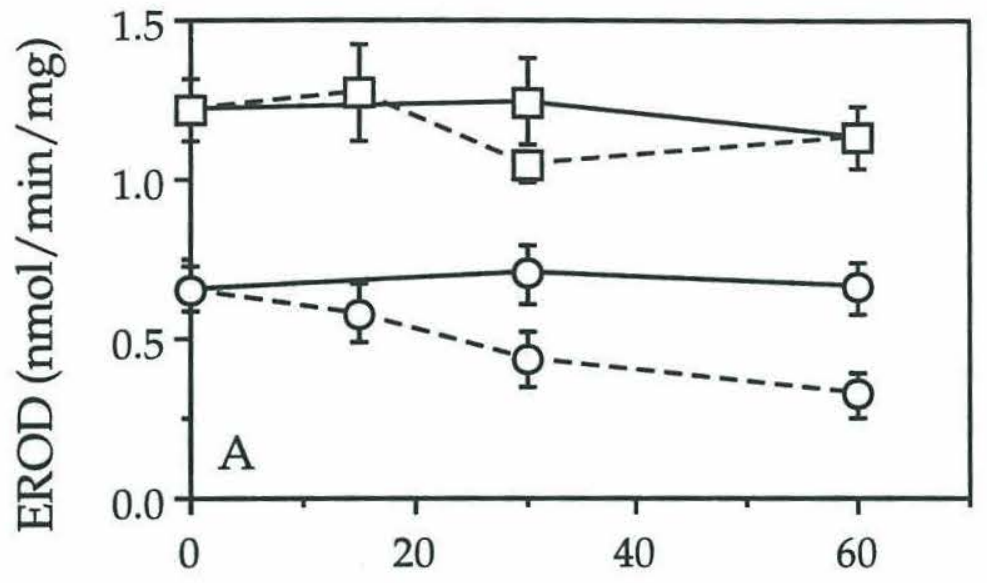

\begin{tabular}{|ll}
\hline$-\square-$ & control \\
\hline- N- & NADPH \\
$--0-$ & TCB \\
& TCB+NADPH
\end{tabular}

Figure 10. CYP1A1 inactivation in the presence of azide in vitro. Hepatic microsomes from feral scup were incubated + or - substrate $(1.5 \mu \mathrm{M}),+$ or - NADPH cofactor, and + or - sodium azide ( $1 \mathrm{mM}$ ), and aliquots of the incubations were removed at $0,10,20$, and 30 minutes for fluorometric measurement of residual EROD activity in microsomes. (A) Microsomal incubation without substrate. (B) Microsomal incubation with $1.5 \mu \mathrm{M}$ TCB. (C) Microsomal incubation with $1.5 \mu \mathrm{M} \mathrm{BaP}$. X-axis is the length of time of microsomal incubations, $y$-axis is the rate of EROD activity in the incubations at given time points. Each point represents the mean EROD rate of duplicate assays from a single experiment. Each experiment was conducted twice, giving similar results. 

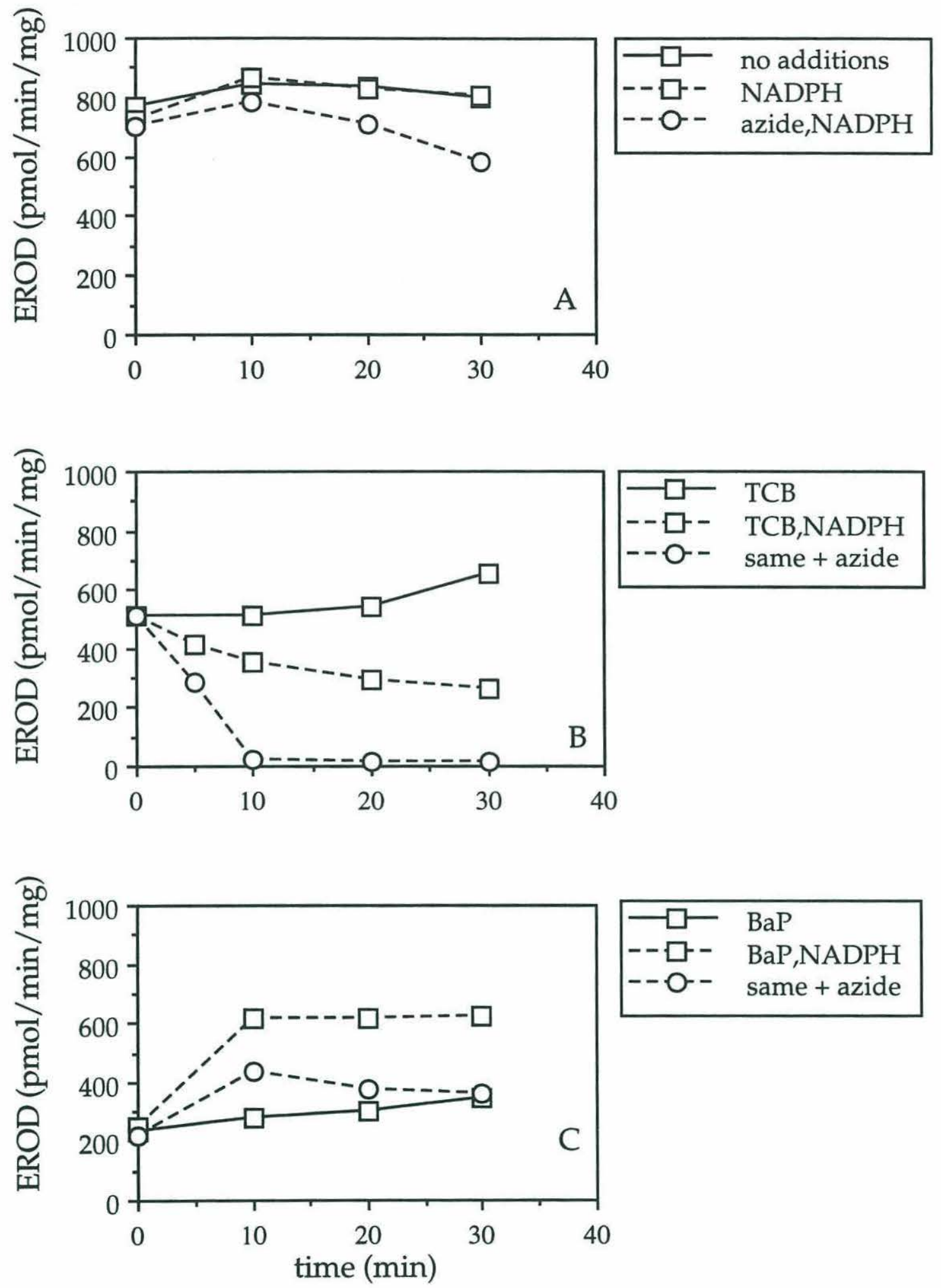


\section{Discussion}

The apparent inhibition of CYP1A catalytic activity by the Ah receptor agonist 3,3',4,4'-TCB was first demonstrated in studies of rainbow trout hepatic microsomes (Melancon and Lech, 1983). Here we provide evidence demonstrating that the strong negative effect of 3,3',4,4'-TCB on hepatic CYP1A1 activity involves suppression of CYP1A1 protein content. Lower doses of TCB $(0.1 \mathrm{mg} / \mathrm{kg})$ elicit transcriptional activation of CYP1A1 in scup, with elevated CYP1A1 mRNA content that is followed in time by elevations in CYP1A1 enzyme content and CYP1A1 enzyme activity, consistent with previous observations of CYP1A1 induction in teleosts (e.g. Kloepper-Sams and Stegeman, 1989). At higher doses $(5.0 \mathrm{mg} / \mathrm{kg})$, TCB elicits a similar transcriptional activation, with elevated CYP1A1 mRNA expression at early time points, but this is followed by only a very weak induction of CYP1A1 enzyme content and activity. This effect, which is dose-dependent in vivo, is shown further to be highly specific for CYP1A1, and in vitro experiments suggest that it may involve inactivation of CYP1A1 by oxidation of the $\mathrm{P} 450$ active site.

\section{Specificity of suppression by TCB in vivo}

Whereas high doses of TCB elicited suppressed microsomal content of CYP1A1 protein, many other microsomal proteins were not negatively affected by the high TCB dose. Rather, content of cytochrome b5, NADPH P450 reductase, and UGT were each elevated to the same extent by both TCB doses. The turnover rate of NADPH P450 reductase in mammals, 48-72 hours (Ernster and Orrenius, 1965; Omura et al., 1967; Arias et al., 1969; Kuriyama et al., 1969), is similar to that of teleost CYP1A1, 39 hours (Kloepper-Sams and Stegeman, 1994); the turnover rate of cytochrome b5 in mammals, 100 hours (Okada and Omura, 1978), is approximately twice as long. Any general destabilization would likely have affected expression of all of these enzymes, as they are similarly located and have comparable half-lives. The fact that TCB did not affect expression of these enzymes indicates that it does not act by a general mechanism, such as cellular toxicity, membrane degradation, accelerated turnover of all microsomal protein, or reduced translation of all cellular mRNA. Histopathological examination of the livers of these TCB-treated fish indicated no degenerative or necrotic damage, and all histological alterations seen were similar at the two TCB doses (chapter 4), further suggesting that TCB was not acting as a general hepatotoxicant at the high dose.

TCB also had minimal effect on the expression and activity of other cytochromes P450. Although the content of total cytochrome P450 was decreased at the high TCB dose 
as compared to the low dose, the negative effect on CYP1A1 can account for the majority of that decline (Table 1) Prior investigations of fish treated with polychlorinated aromatics have also noted that a large portion of the P450 induction is accounted for by CYP1A1 induction (Gooch et al., 1989; Hahn and Stegeman, 1994). Content of the putative scup CYP2B and the putative scup CYP3A were somewhat lower in fish treated with $5.0 \mathrm{mg} / \mathrm{kg}$ as compared to those treated with $0.1 \mathrm{mg} / \mathrm{kg}$, but the differences between treatments were of a very small magnitude compared to CYP1A1 (y-axes, Fig 2), and the wide range of values within each treatment group made it difficult to define a specific "TCB effect". Nonetheless, it is possible that the mechanisms which cause suppressed CYP1A1 protein content (discussed below) may exert a weak effect on other P450 enzymes as well.

In a previous study (Gooch et al., 1989), as in the present study, scup treated with $5.0 \mathrm{mg} / \mathrm{kg}$ TCB had significantly lower rates of EROD activity on day 5 than did scup treated with a lower TCB dose $(1.0 \mathrm{mg} / \mathrm{kg})$. However, the rates of aminopyrine $N$ demethylase (APND) activity and estradiol 2-hydroxylase (E2OH) activity were similar on day 5 at the two TCB doses (Gooch et al., 1989). The monooxygenase activities APND and E2OH are not catalyzed by CYP1A1 in scup (Stegeman et al., 1985; Snowberger and Stegeman, 1987), further indicating that the suppressive effect of TCB is specific for the CYP1A1 protein.

\section{Dose dependence of suppression by TCB in vivo}

The induction and suppression of CYP1A1 protein were related to the concentration of TCB in liver. In fish administered $0.1 \mathrm{mg} / \mathrm{kg} \mathrm{TCB}$, the level of CYP1A1 induction was directly correlated with the amount of TCB in liver. Thus, the maximum TCB concentration on day 5 coincided with maximum CYP1A1 levels on day 5 , and on days 12 and 16 both TCB content and CYP1A1 expression were diminished. In fish administered $5.0 \mathrm{mg} / \mathrm{kg}$ TCB, hepatic TCB content was relatively high on days 5 and 12 ( 0.6 and 2.2 $\mu \mathrm{g} / \mathrm{g}$ ) and CYP1A1 content was apparently suppressed at these time points, whereas on day 16 hepatic TCB content was reduced in the pooled sample $(0.4 \mu \mathrm{g} / \mathrm{g})$ and CYP1A1 content and activity were elevated in two of the four fish. Thus, TCB depuration seems to be associated with a release of EROD inhibition, with increased content of CYP1A1 mRNA, and with a slightly increased content of CYP1A1 protein, in two fish sampled on day 16. The elevated level of CYP1A1 mRNA following depuration suggests that TCB may suppress CYP1A1 expression at a transcriptional level, as has been indicated in other studies (Wirgin et al., 1992; Celander and Forlin, submitted). In the present study, however, transcriptional regulation cannot account for the suppressed levels of CYP1A1 protein on days 2 through 12 . 
The residue data suggest that suppression of CYP1A1, at multiple levels (a pretranslational level, the level of protein content, and the level of enzyme activity), is linked to TCB concentrations in excess of $0.4 \mu \mathrm{g} \mathrm{TCB} / \mathrm{g}$ liver dry weight, or $0.1 \mu \mathrm{g} \mathrm{TCB} / \mathrm{g}$ liver wet weight. This value is approximate, as TCB content was measured in pooled samples, and inter-individual variation in hepatic TCB content was likely. Gooch et al. (1989) reported inhibition of microsomal EROD activity in scup livers with TCB concentrations exceeding $2 \mu \mathrm{g} / \mathrm{g}$ wet weight; our results suggest an even more sensitive system. Hepatic concentrations of TCB in feral fish from highly contaminated sites have been reported in the $0.1 \mu \mathrm{g} / \mathrm{g}$ wet weight range. Winter flounder from New Bedford Harbor, MA were reported to have TCB concentrations ranging from 0.009 to $0.263 \mu \mathrm{g} / \mathrm{g}$ liver wet weight (Monosson and Stegeman, 1991). Our data indicate that TCB could elicit suppression of CYP1A1 content or activity in fish living in polluted areas, as has been suggested previously (Elskus et al., 1989; Monosson and Stegeman, 1991). Such suppression may compromise the animal's ability to effectively metabolize other pollutant compounds that are CYP1A1 substrates and that co-occur with TCB in the environment, including polycyclic aromatic hydrocarbons.

\section{Mechanism of post-transcriptional suppression}

The decreased content of both immunodetectable CYP1A1 protein and spectrally detectable P450 holoenzyme in TCB-treated fish suggests the possible involvement of several mechanisms: (A) reduced CYP1A1 mRNA translation; $(\mathrm{B})$ reduced synthesis of the P450 heme moiety; or (C) enhanced CYP1A1 protein degradation. A previous study of TCBtreated scup (Gooch et al., 1989) found that CYP1A1 mRNA isolated on day 5 from fish treated with $5.0 \mathrm{mg} / \mathrm{kg}$ TCB was competent for in vitro translation. Furthermore, Northern blot analysis (Fig 5) showed that the electrophoretic migration of the CYP1A1 mRNA was the same at the two doses. It thus appears that scup treated with a high TCB dose express a full and translatable CYP1A1 message, arguing against reduced translation (A).

TCB exposure has previously been reported to inhibit heme synthesis in chick embryo hepatocytes (Sassa et al., 1986; Sinclair et al., 1986). However, in the present study CYP1A1 was the only microsomal heme protein with strongly reduced content at the high TCB dose as compared to the low dose. Cytochrome b5 was equally elevated at the two doses, and content of total cytochrome P450 never dropped below basal levels in fish treated with the high dose, suggesting that a "constitutive" P450 pool was unaffected by TCB. Sustained hepatic concentrations of the latter hemoproteins argues against a depleted heme pool to explain reduced CYP1A1 content (B). 
Evidence from both in vivo and in vitro studies suggests that TCB may elicit enhanced CYP1A1 protein degradation (C), which could explain the reduced CYP1A1 content seen in fish treated with $5.0 \mathrm{mg} / \mathrm{kg}$ TCB. Firstly, the strong in vivo suppression by TCB was specific for CYP1A1, which is a principal catalyst for the metabolism of TCB in scup hepatic microsomes (chapter 5). A reduced enzyme content at the high TCB dose was not seen for proteins that are apparently co-induced with CYP1A1 (cytochrome b5, UGT), nor was strong suppression seen for other cytochromes P450, consistent with the suppression being related to TCB metabolism or active-site binding. Secondly, the in vivo suppression by TCB was only seen in epithelial cells of liver, kidney, and gill; endothelia of all organs examined had an equally strong CYP1A1 induction at the two TCB doses (chapter 3). Prior investigations (Stegeman et al., 1982; Stegeman et al., 1989) indicate that the ratio of NADPH cytochrome $\mathrm{c}$ reductase : cytochrome $\mathrm{P} 450$ is one order or magnitude lower in scup heart (where all of the P450 is endothelial) than in scup liver (where most of the P450 is epithelial). Limiting quantities of reductase enzyme in endothelia may be expected to result in lower P450 catalytic activity in these cells, even with highly induced P450 content. In the present study, the "suppressed" P450 content in epithelia, but not in endothelia, is consistent with suppression being dependent upon electron transfer to P450, which may be greater in the epithelial cells due to abundant reductase.

If the reduced CYP1A1 content in vivo is indeed the result of enzyme degradation rather than decreased enzyme biosynthesis, one may expect to see CYP1A1 degradation products in immunoblots. The fact that we did not see such products may indicate either (1) the principal degradation products are not recognized by the anti-CYP1A1 MAb 1-12-3, (2) the quantities of such products are below the limits of detection of the assay used, or (3) the protein is rapidly broken down into amino acids or small peptides upon synthesis. The latter may involve modification of the enzyme, during interaction with TCB, in such a way as to mark it for proteolysis.

Evidence from in vitro studies further supports the hypothesis that suppressed CYP1A1 content in vivo is related to TCB metabolism or active-site binding. Hepatic microsomal P450 is denatured (Fig 8) and CYP1A1 is inactivated (Fig 9A) in the presence of TCB plus cofactor. We speculate that the majority of the spectral P450 loss under this condition was due to CYP1A1 loss. The requirement of both TCB and cofactor for the inactivation and denaturation implies a process involving electron transfer to and TCB binding in the CYP1A1 active site. The fact that 2,2',5,5'-TCB, which binds weakly if at all to the scup CYP1A1 active site (Gooch et al., 1989) and is not a substrate for scup CYP1A1 (chapter 5), did not inactivate CYP1A1 in vitro (Fig 9C) further supports the 
suggestion that inactivation requires active site binding. Inactivation of the enzyme during substrate-binding and electron transport may be caused by either a reactive TCB metabolite formed in the active site (mechanism-based inactivation), or a reactive oxygen species generated in the active site (oxidative inactivation), as discussed below.

\section{Mechanism-based inactivation}

In mechanism-based inactivation, substrate is metabolized by $\mathrm{P} 450$ to a reactive intermediate that can form covalent attachments to the P450 apoenzyme or to the heme moiety, irreversibly inhibiting enzyme activity (Ortiz de Montellano and Reich, 1986). Studies with rat hepatic microsomes and reconstituted systems(Shimada and Sawabe, 1983) have shown that TCB is metabolically activated by CYP1A enzyme(s) to reactive form(s) that binds covalently to cellular proteins, including CYP1A. Similarly, we detected covalent binding to rat microsomal protein of TCB metabolites generated in rat microsomes, but the rate of TCB activation and binding in scup hepatic microsomes was very low (Table 2). At the rate measured in microsomes from feral scup, reactive TCB metabolites would be expected to affect only $3 \%$ of the microsomal CYP1A1 pool in one hour, even if one assumes that all of the covalent binding seen was to CYP1A1. Furthermore, spectral analysis of microsomal P450 following incubation with TCB plus NADPH indicated that there was no heme loss from the enzyme (Fig 8), excluding the possibility that TCB formed covalent adducts with the $\mathrm{P} 450$ heme that went undetected due to heme loss. Thus mechanism-based inactivation cannot account for the rapid CYP1A1 inactivation seen in vitro (Table 3 ).

It is nonetheless noteworthy that TCB conversion to active metabolites (those that can form covalent attachments to cellular proteins) comprises approximately $16 \%$ of the total TCB metabolism in feral scup microsomes: aqueous-soluble metabolites are generated at a rate of approximately $7.8 \mathrm{pmol} / \mathrm{hr} / \mathrm{mg}$ (chapter 5), and protein-bound metabolites at approximately $1.5 \mathrm{pmol} / \mathrm{hr} / \mathrm{mg}$. Despite such slow metabolism in vitro, appreciable amounts of TCB metabolites have been detected in the bile of TCB-treated scup (White et al., in preparation). Thus reactive metabolite formation and mechanism-based inactivation may play some role in CYP1A1 depletion in vivo. The very slow rate of metabolism in vitro further indicates that an aqueous-soluble TCB metabolite is not responsible for the rapid CYP1A1 inactivation and denaturation seen in microsomes.

\section{Oxidative inactivation}

Oxidative inactivation of $\mathrm{P} 450$ has been associated with catalytic uncoupling, occurring when electron transport is uncoupled from monooxygenation. This is seen when a poorly 
metabolized substrate or unmetabolized pseudosubstrate binds to the $\mathrm{P} 450$ active site, stimulating electron flow to and oxygen activation in the active site as would a well-coupled substrate, but the activated oxygen is incorporated into substrate at a low or negligible rate (Ullrich and Diehl, 1971; Staudt et al., 1974). Some of the $\mathrm{O}_{2}$ that is activated but is not utilized for substrate monooxygenation can then be converted to $\mathrm{H}_{2} \mathrm{O}_{2}$ (Nordblom and Coon, 1977; Loosemore et al., 1980; Kuthan and Ullrich, 1982), via a direct breakdown of the activated P450-peroxy complex (Karuzina and Archakov, 1994). $\mathrm{H}_{2} \mathrm{O}_{2}$ may be converted to other reactive oxygen species including hydroxyl radical, which are believed to cause oxidative modifications to either the P450 apoenzyme or to the heme, ultimately leading to accelerated enzyme degradation (Karuzina and Archakov, 1994).

The CYP1A1 inactivation and denaturation due to TCB plus NADPH were greatly enhanced upon addition of sodium azide (Table 3). Azide is an inhibitor of catalase activity and thus prevents the degradation of $\mathrm{H}_{2} \mathrm{O}_{2}$ that is produced in microsomes. Azide has also been found to be converted to azidyl radical, in the presence of hydroxyl radical (Reinke et al., 1994). Azidyl radical may be formed in or near the $P 450$ active site, and may be capable of directly attacking the enzyme, along with the reactive oxygen species present. Thus azidyl radical may be acting to enhance and accelerate the destructive process that would normally be carried out by oxygen radicals alone. The apparent destructive action of azide may therefore involve both catalase inhibition and azidyl radical formation. Irrespective of which azide mechanism predominates, this result implies that reactive oxygen species are generated in microsomes as reducing equivalents are passed to the CYP1A1/TCB complex. Enzyme inactivation and denaturation due to azide were comparatively slight in the absence of substrate, or in the presence of the rapidly metabolized substrate $\mathrm{BaP}$ (Fig 10), consistent with prior work showing that some $\mathrm{H}_{2} \mathrm{O}_{2}$ production occurs in microsomes in the absence of substrate or in the presence of a tightlycoupled substrate (Nordblom and Coon, 1977; Guengerich, 1978; Kuthan and Ullrich, 1982).

In further support of our interpretations, De Matteis and colleagues (De Matteis et al., 1989; De Matteis et al., 1991; De Matteis et al., 1993) have reported that addition of TCB plus NADPH to hepatic microsomes, after treatment with CYP1A1 inducers, stimulated the oxidative degradation of bilirubin. These investigators, too, have postulated a mechanism involving catalytic uncoupling of CYP1A1 by TCB binding, and have postulated that reactive oxygen species may be responsible for the bilirubin degradation. The latter studies have not addressed effects on P450 itself. In the present study, experiments with azide provide strong evidence for the generation of reactive oxygen species in the TCB/NADPH/CYP1A1 microsomal system, which target CYP1A1 itself for 
denaturation. It is interesting to note that reactive oxygen, presumably synthesized in the CYP1A1 active site, can target both CYP1A1 and bilirubin for degradation, but apparently does not affect cytochrome b5, UGT, or NADPH reductase in vivo (this study).

Exogenous catalase did not offer protection from enzyme inactivation by TCB plus NADPH. Similar findings have been made by other investigators studying catalytic uncoupling (Loosemore et al., 1980). This may imply that the destructive agent directly attacking P450 is a reactive oxygen species other than $\mathrm{H}_{2} \mathrm{O}_{2}$, for example hydroxyl radical, which is formed from $\mathrm{H}_{2} \mathrm{O}_{2}$ in the metal-catalyzed Fenton reaction (Halliwell and Gutteridge, 1986). The apparently paradoxical azide/catalase results may then be explained as follows: if hydroxyl radical is formed rapidly in the active site from $\mathrm{H}_{2} \mathrm{O}_{2}$, then additional $\mathrm{H}_{2} \mathrm{O}_{2}$ degradation in the medium by exogenous catalase may not affect enzyme inactivation; however, azide inhibition of the endogenous catalase in the medium may lead to $\mathrm{H}_{2} \mathrm{O}_{2}$ accumulation in the medium, and hence inhibited flow of $\mathrm{H}_{2} \mathrm{O}_{2}$ from the active site into the medium due to a loss of the concentration gradient, thus enhancing hydroxyl radical formation in the active site and consequent enzyme inactivation. Alternatively, it is possible that azide is not acting as a catalase inhibitor in this system, but is simply interacting with the destructive hydroxyl radicals in the active site to form a second destructive species in the active site, azidyl radical. Hydroxyl radical is highly reactive and will thus combine at or very close to its site of formation (Halliwell and Gutteridge, 1986). It will be formed from $\mathrm{H}_{2} \mathrm{O}_{2}$ in vivo at the points in the cell where metal catalysts of the Fenton reaction are located. Thus biological damage from $\mathrm{H}_{2} \mathrm{O}_{2}$ that is mediated by hydroxyl radical will only occur at or near metal ion complexes that catalyze the Fenton reaction (Halliwell and Gutteridge, 1986). It is interesting to speculate that the cytochrome P450 heme may act as a Fenton catalyst in vitro and in vivo, although this has not yet been demonstrated.

Characteristic of an uncoupler, TCB was found to be a tightly-bound but poorly metabolized substrate for scup CYP1A1 in vitro (chapter 5). Interestingly, Hesse and Wolff (1977) reported catalytic uncoupling during the binding of the pseudosubstrate $2,2^{\prime}, 4,4$ ',6,6'-hexachlorobiphenyl, and of the poorly metabolized substrate 2,2',5,5'tetrachlorobiphenyl, to hepatic microsomal cytochrome $\mathrm{P} 450$ from phenobarbital-treated rabbits. We speculate that the slow, uncoupled metabolism of the higher chlorinated biphenyls may be due to steric hindrance from the bulky chlorine substituents on each side of the molecule, which could block oxygenation at the adjacent, unsubstituted carbon atoms. Hindered oxygenation of substrate could then result in prolonged substrate occupation of the active site, and in the production of reactive oxygen species during such occupation, which may in turn inactivate the enzyme. While oxidative CYP1A1 
inactivation would be expected to occur at any concentration of TCB, it may not be detectable in vivo in livers with a high CYP1A1:TCB ratio (i.e. in animals receiving lower TCB doses).

In summary, we have demonstrated that 3,3',4,4'-TCB brings about a strong, transcriptional induction of scup CYP1A1, acts as a reversible inhibitor of CYP1A1 catalytic activity, and simultaneously elicits a post-translational suppression of CYP1A1 protein content in vivo. This suppression appears to involve direct interactions between TCB and CYP1A1, and in vitro evidence strongly implicates oxidative inactivation in the reduced CYP1A1 protein content. Given the strong inactivation, TCB may be a useful selective inhibitor in establishing CYP1A1 catalytic activities. Furthermore, our results establish that TCB should not be utilized as a simple CYP1A1 inducer in in vivo studies, as host of other complex interactions also take place. 


\title{
CHAPTER THREE
}

\author{
Temporal Pattern of Induction of Cytochrome P4501A1 \\ in Multiple Organs and Cell Types of the Marine Fish Scup \\ Treated with Low and High Doses of 3,3',4,4'-Tetrachlorobiphenyl
}




\section{Abstract}

Cytochrome P4501A1 (CYP1A1) plays an important role in both the metabolism and the activation of organic contaminants, including polycyclic aromatic hydrocarbons and halogenated aromatic hydrocarbons. Regulation of CYP1A1 has been studied extensively in teleost liver, but very little is known about the dose-response and time-course patterns of CYP1A1 induction in target cells of extrahepatic organs of fish. In the present study the marine fish scup (Stenotomus chrysops) was treated with the CYP1A1 inducer 3,3',4,4'tetrachlorobiphenyl (TCB) at two doses, $0.1 \mathrm{mg} / \mathrm{kg}$ and $5.0 \mathrm{mg} / \mathrm{kg}$, and animals were sampled at time points from 1 to 16 days after treatment. Sections of liver, kidney, gill, intestine, spleen, heart, and gonad were analyzed for CYP1A1 content with immunohistochemistry, and microsomes from liver, kidney, and gill were analyzed for CYP1A1 content by immunoblot. Immunoblot analysis showed strong CYP1A1 induction in liver, kidney, and gill of animals treated with $0.1 \mathrm{mg} / \mathrm{kg} \mathrm{TCB}$, and a comparatively weaker induction throughout the time-course in liver, kidney, and gill of animals treated with $5.0 \mathrm{mg} / \mathrm{kg}$ TCB. In immunohistochemistry, vascular endothelium of all organs showed CYP1A1 induction by day 1 , which reached a maximum of intensity and prevalence on day 12 , and did not attenuate by the end of the experiment. This temporal pattern of induction in endothelium did not differ between the two TCB doses. Fish treated with the high dose had greater CYP1A1 induction in mucosal epithelium of both duodenal and caecal sections of intestine than did fish treated with the low dose, reflecting higher inducer concentration passing through the intestine. Spleen of fish treated with both doses had strong CYP1A1 induction in vascular endothelia of major arterioles, and spleen of fish treated with the high dose also had moderate induction in the capillary endothelia.

Similarly, both doses of TCB elicited a strong CYP1A1 induction in the endocardia of major vessels of the bulbus arteriosus, and the high dose also elicited a weak induction in capillary endothelia of the bulbus. In some epithelial cell types, CYP1A1 induction was greater in fish treated with $0.1 \mathrm{mg} / \mathrm{kg}$ TCB than in fish treated with $5.0 \mathrm{mg} / \mathrm{kg} \mathrm{TCB}$, at certain time points: hepatocytes, renal tubules, renal collecting ducts, and gill epithelia. In kidney and gill, the "reduced" CYP1A1 content at the high dose reflected a reduced percentage of strongly induced cells, whereas the "reduced" induction in liver at the high dose reflected a weaker CYP1A1 staining in all hepatocytes, as compared to the low dose. The apparent suppression of induction in kidney and gill of fish treated with $5.0 \mathrm{mg} / \mathrm{kg}$ TCB may be related to degree of tissue pathology, which was more severe in these organs at the high dose. Reduced CYP1A1 content in liver seems to be related to a specific interaction between TCB and CYP1A1, rather than to general pathology. 


\section{Introduction}

Cytochrome P4501A1 (CYP1A1) plays a critical role in the activation and detoxification of a large group of lipophilic organic contaminants, including polycyclic aromatic hydrocarbons and halogenated aromatic hydrocarbons (Stegeman and Hahn, 1994). The binding of planar aromatic hydrocarbons to the cytosolic Ah receptor in mammals mediates a transcriptional induction of CYP1A enzymes, resulting in increased content of CYP1A proteins in the endoplasmic reticulum, and increased rates of metabolism of substrates/ inducers (Nebert and Gonzalez, 1987). The identification of hepatic Ah receptor in fish (Hahn et al., 1994), and the similarity in timing of the induction response between teleost and mammalian hepatic CYP1A1 (Kloepper-Sams and Stegeman, 1989), indicates that a similar mechanism of induction operates in teleosts. Although CYP1A1 induction has been studied extensively in teleost liver, less is known about the responses of extrahepatic CYP1A1 to Ah receptor agonists, and in particular very little is known about the time-course and dose-response patterns of induction in target cells of extrahepatic organs. Extrahepatic CYP1A1 induction may prove to play a critical role in both the detoxification and the activation of environmental substrates in fish, particularly in organs such as gill and intestine that are directly exposed to the environment.

CYP1A1 enzymatic activity has been documented in most organs of fish, including liver, kidney, gonad, intestine, gill, heart, muscle, and others (Stegeman et al., 1979). Exposure to Ah receptor agonists has been found to increase rates of microsomal CYP1A1 activity in several teleost organs, including liver and kidney (Bend et al., 1974), intestine (Van Veld et al., 1988), gill (Miller et al., 1989), and heart (Stegeman et al., 1989). Induction of CYP1A1 protein has been documented in multiple organs of fish exposed to Ah receptor agonists, including liver, kidney, gill, heart, intestine, gonad, spleen, brain, and olfactory epithelium (Lorenzana et al., 1988; Miller et al., 1988; Miller et al., 1989; Stegeman et al., 1989; Smolowitz et al., 1991; Smolowitz et al., 1992; Buchmann et al., 1993; Lindstrom-Seppa et al., 1994). In many extrahepatic organs, the major site of induction has been identified as vascular endothelium (Miller et al., 1989; Stegeman et al., 1989; Smolowitz et al., 1991; Stegeman et al., 1991), specifically in gill, heart, gonad, spleen, and brain. In some organs, including liver, kidney, gill, and intestine, induction has also been observed in epithelial cell types (Smolowitz et al., 1991; Smolowitz et al., 1992; Lindstrom-Seppa et al., 1994). CYP1A1 induction in multiple organs has also been observed in chronically exposed fish from the wild (Stegeman et al., 1991), with vascular endothelium being the most common site of induction in these fish. 
A recent study (Smolowitz et al., 1991) has provided a thorough

immunohistochemical survey of CYP1A1 induction in multiple organs and cell types of the marine fish scup (Stenotomus chrysops) treated with 3,3',4,4'-tetrachlorobiphenyl (TCB). TCB is an Ah receptor agonist that acts as a CYP1A1 inducer, a CYP1A1 inhibitor (Gooch et al., 1989), and a CYP1A1 substrate (chapter 5) in teleost fish. TCB is a component of commercial polychlorinated biphenyl (PCB) mixtures, which occur as ubiquitous contaminants in marine environments (Tanabe, 1988; Fowler, 1990). TCB and the other coplanar PCB congeners bring about a suite of toxic effects in mammals, similar to those elicited by 2,3,7,8-tetrachlorodibenzo- $p$-dioxin (reviewed in Goldstein and Safe, 1989). The study of Smolowitz and colleagues (Smolowitz et al., 1991) described induction at only a single time-point after treatment (day 5), and in response to only a single high TCB dose $(1 \mathrm{mg} / \mathrm{kg})$. The present study extends these findings by describing the degree of CYP1A1 induction from day 1 to day 16 after treatment, in several cell types of 7 organs in scup, in response to two different doses of TCB: $0.1 \mathrm{mg} / \mathrm{kg}$ and $5.0 \mathrm{mg} / \mathrm{kg}$. We report that strong induction is evident by day 1 in some cell types, including atrial endocardia (both doses) and gill epithelia (high dose). Induction attenuates by day 16 in some epithelial cell types, particularly at the low dose, but is sustained in the vascular endothelium of all organs examined. Induction was stronger at the high dose than at the low in mucosal epithelium of intestine, and capillary endothelium of spleen and bulbus arteriosus.

However, the low dose elicited a stronger overall induction than did the high dose in gill epithelia, renal tubules and collecting ducts, and hepatocytes.

\section{Materials and Methods}

Chemicals 3,3',4,4'-Tetrachlorobiphenyl (TCB, IUPAC \#77) was purchased from Pathfinder (St. Louis, MO). Purity of TCB was greater than $99 \%$ by GC/ECD and GC/MS analysis. Further analysis by high resolution ( 5000 resolution) mass spectrometry (VG Autospec) and selective ion monitoring showed that the potent Ah receptor agonist 3,3',4,4',5-pentachlorobiphenyl was not a contaminant of the TCB, with a detection limit of $0.001 \%$. For immunohistochemistry, the Universal Immunoperoxidase Staining Kit (Murine) (Signet Laboratories, Dedham, MA) was used. All other immunohistochemical reagents were similar to those used previously in this laboratory (Smolowitz et al., 1991). Reagents for biochemistry were of the highest grade available from commercial suppliers.

Animals and Dissection Scup (Stenotomus chrysops) were caught by angling in Vineyard Sound, MA in August 1991. Fish were held in flowing seawater tanks at $20^{\circ} \mathrm{C}$ for 
fourteen months prior to experimental use, and were maintained on a diet of Purina trout chow and frozen squid. Prior studies in our laboratory indicate that the bulk of environmental contaminants are depurated from the tissues of fish that are held in aquaria for one year, and that these fish show little CYP1A1 expression. At the time of the dosing, experimental animals (mixed sex) were gonadally immature and weighed 76 - 276 grams. Individuals were injected intraperitoneally with either corn oil (carrier controls) or with 0.1 $\mathrm{mg} / \mathrm{kg}$ or $5.0 \mathrm{mg} / \mathrm{kg} 3,3^{\prime}, 4,4^{\prime}$-tetrachlorobiphenyl (TCB) dissolved in corn oil. A group of uninjected fish was used as a "zero" point in the time-course. Individuals were held in flowing seawater tanks at $20^{\circ} \mathrm{C}$ until sampling (1 day to 18 days) or until death from toxicity. Fish were fed Purina trout chow once weekly, and were never fed within the three days prior to killing. At each time point, a group of 4 or 5 animals from each treatment group were killed by cervical transection and dissected immediately.

Sections of liver, kidney, and gill for biochemistry were removed from the fish and frozen in liquid nitrogen within one to five minutes of death. Tissues were held in liquid nitrogen until microsome preparation. Prior studies in our laboratory indicate that enzyme degradation does not occur to any appreciable degree in scup tissue stored at liquid nitrogen temperatures (unpublished observations). Microsomal fractions were prepared from frozen liver, kidney, and gill by differential centrifugation, as previously described (Hahn and Stegeman, 1994), and microsomal protein content was measured with the bicinchoninic acid method (Smith et al., 1985), using bovine serum albumin as a standard. Sections of liver, posterior kidney, gonad, heart, gill, spleen, and intestinal caecum and duodenum were removed and immediately fixed in $10 \%$ neutral buffered formalin ( $\mathrm{pH} 7.4$ ). Spleen was only sampled on days 0,12 , and 16 ; all other organs were sampled at each time point. After one week of fixation, tissues were embedded in paraffin using standard methods (Luna, 1968).

Biochemistry For biochemical analyses, a hepatic microsomal fraction was prepared from each individual fish. Kidney and gill microsomal fractions were obtained from tissue pieces of equal size from all fish within a given treatment group (i.e. dose and time point), and pooled for analyses. Microsomal fractions from liver, kidney, and gill were analyzed for content of cytochrome P4501A1 (CYP1A1) by Western blot analysis, as described previously (Kloepper-Sams et al., 1987; Stegeman et al., 1991). Antibodies used were monoclonal antibody 1-12-3 (Park et al., 1986), raised against scup P450E (a CYP1A1 homologue), and alkaline phosphatase linked goat-anti-mouse IgG as the secondary antibody. Relative content of CYP1A1 in the microsomal samples was measured by densitometric scanning of immunoreactive bands with a video imaging system (Masterscan, 
Scanalytics, division of CSPI, Billerica, MA), and comparison of optical density in the samples to optical density in standards with known P450E content.

Microsomal fractions from liver, kidney, and gill were analyzed for rates of ethoxyresorufin O-deethylase (EROD) activity, which is catalyzed by CYP1A1 in scup (Stegeman et al., 1985). EROD activity was measured at $32^{\circ} \mathrm{C}$ with a fluorometric kinetic assay, using a Cytofluor 2300 fluorescence plate reader (Millipore), as described previously (Hahn et al., 1993). Protein concentration in the EROD assays was approximately $0.05 \mathrm{mg} / \mathrm{ml}$ for liver ( $1 \mu \mathrm{l}$ hepatic microsomal protein per $200 \mu \mathrm{l}$ assay), $0.05-0.3 \mathrm{mg} / \mathrm{ml}$ for kidney, and $0.2-0.7 \mathrm{mg} / \mathrm{ml}$ for gill $(10 \mu \mathrm{l}$ kidney or gill microsomal protein per $200 \mu \mathrm{l}$ assay).

Immunohistochemistry Paraffin blocks were chilled in ice water, and $5 \mu \mathrm{m}$ sections were obtained, mounted on Superfrost-plus slides (Baxter), and allowed to dry at room temperature for at least 24 hours. Tissue sections on slides were deparaffinated in clearant (Americlear histology clearing solvent) for 6 minutes. Tissues were then hydrated successively in $100 \%$ ethanol ( $2 \mathrm{~min}$ ), methanol containing $0.5 \%$ hydrogen peroxide (to block endogenous peroxidase, $45 \mathrm{~min}$ ), $95 \%$ ethanol ( $2 \mathrm{~min}), 80 \%$ ethanol ( $1 \mathrm{~min}), 70 \%$ ethanol ( $1 \mathrm{~min}$ ), water (15 min), and finally $1 \% \mathrm{BSA} / \mathrm{PBS}(\geq 5 \mathrm{~min})$. Hydrated tissue sections were stained using an indirect peroxidase stain (Universal Immunoperoxidase Staining Kit (Murine), Signet Laboratories, Dedham, MA) with MAb 1-12-3 as the primary antibody. This antibody has been found to bind specifically to CYP1A1 in tissue sections (Miller et al., 1989).

After hydration in $1 \%$ BSA/PBS, slides were soaked for 20 minutes in normal goat serum, to block nonspecific attachment of the secondary antibody (goat anti-mouse IgG). Slides were then incubated in MAb 1-12-3 (1 $\mu \mathrm{g} / \mathrm{ml})$ in 1\% BSA/PBS for two hours; negative control slides were incubated in a non-immune serum, specifically mouse myeloma IgG (1.8 $\mu \mathrm{g} / \mathrm{ml}$; UPC-10, IgG $2 \mathrm{~A}$, Organon Teknika, West Chester, PA) in 1\% BSA/PBS for 2 hours. These incubations were followed by one wash with BSA/PBS, and a 20 minute incubation with secondary antibody, goat anti-mouse IgG. Slides were washed again in BSA/PBS, incubated for 20 minutes in peroxidase-linked nonspecific mouse IgG, washed twice more, and exposed to 3-amino-9-ethylcarbazole color developer for 30 minutes. This color developer precipitates a red stain at all immunoreactive sites. Stained sections were washed in water and mounted in $90 \%$ glycerol/PBS. For each organ examined, sections from all 65 fish plus negative control slides were stained together in a single run. 
Scoring of CYP1A1 content CYP1A1 content was quantified in several cell types of seven organs from each fish, by scoring with a subjective index. CYP1A1-specific staining in each cell type was scored for intensity as follows: $0=$ no stain, $1=$ weak, $2=$ moderate, 3 = strong, or "heterogeneous", meaning that populations of cells within a tissue variously stained weakly, moderately, or strongly. CYP1A1-specific staining was scored for prevalence as follows: $0=$ no stain, $1=$ rare cells staining, $2=$ multifocal (many cells or patches of cells) staining, 3 = pervasive (most cells of a given type within the tissue) staining. In most cases, a "score" of CYP1A1 expression was calculated as the product of the intensity and the prevalence scores. In heart there was an exceptionally wide range in intensity of stain, and in all cases staining was pervasive, so endocardia of each chamber were scored for intensity only, from 0 to 5 : $0=$ no stain, $1=$ very weak, $2=$ weak, $3=$ moderate, $4=$ strong, $5=$ very strong.

Photographs $35 \mathrm{~mm}$ color slides (EPY 64 Tungsten Ektachrome) were taken of immunostained microscope slides and scanned into Adobe Photoshop software (v. 2.5.1 for Macintosh) using a Kodak RFS 2035 slide scanner. Images were scaled and labeled using Adobe Photoshop, and were printed on a Kodak XL photographic printer. No image enhancement, contrast adjustment, or color adjustment was used to modify the figures.

\section{Results}

Microsomal biochemistry

Content of immunodetectable CYP1A1 was measured in microsomal fractions of kidney and gill from control and TCB-treated scup, and patterns of expression were compared to those in liver microsomes from the same fish (Fig 1). In kidney and liver of fish treated with $0.1 \mathrm{mg} / \mathrm{kg}$ TCB, CYP1A1 induction peaked on day 5 and attenuated by day 16 . In gill microsomes from the same fish, induction peaked later (day 12), and CYP1A1 content seemed to attenuate by day 16 . In fish treated with $5.0 \mathrm{mg} / \mathrm{kg} \mathrm{TCB}$, substantially less CYP1A1 was detected in kidney and liver throughout the time course, and in gill on day 12.

Rates of CYP1A1-catalyzed EROD activity in kidney, gill, and liver microsomes were generally consistent with patterns of CYP1A1 expression (Fig 2). The low dose of TCB brought about a strong induction of activity in all three organs, peaking on day 5 in liver and kidney and on day 12 in gill. In the extrahepatic organs, induction of EROD activity seemed to attenuate by day 16 . In fish treated with the high dose, however, there was minimal EROD induction in kidney, no EROD induction in liver (days 1-12), and 
Fig 1. Immunodetectable CYP1A1 content in kidney, gill, and liver microsomes of TCBand corn oil-treated scup. Microsomal proteins were separated on SDS-PAGE, transferred to nitrocellulose by electroblotting, and incubated with MAb 1-12-3, which was raised against the CYP1A1 homologue from scup liver. $\mathrm{X}$-axis is the number of days subsequent to injection, $\mathrm{y}$-axis is enzyme content in pmol P450E equivalents/ $\mathrm{mg}$ microsomal protein. For kidney and gill, each point is a measurement on a single pooled sample, composed of equally-sized tissue sections from 4 or 5 fish per treatment group. For liver, each point is the mean and standard deviation of measurements on 4 or 5 individuals per treatment group. Plot symbols are: square, corn oil control, open circle, $0.1 \mathrm{mg} / \mathrm{kg} \mathrm{TCB}$, closed circle, $5.0 \mathrm{mg} / \mathrm{kg}$ TCB. 

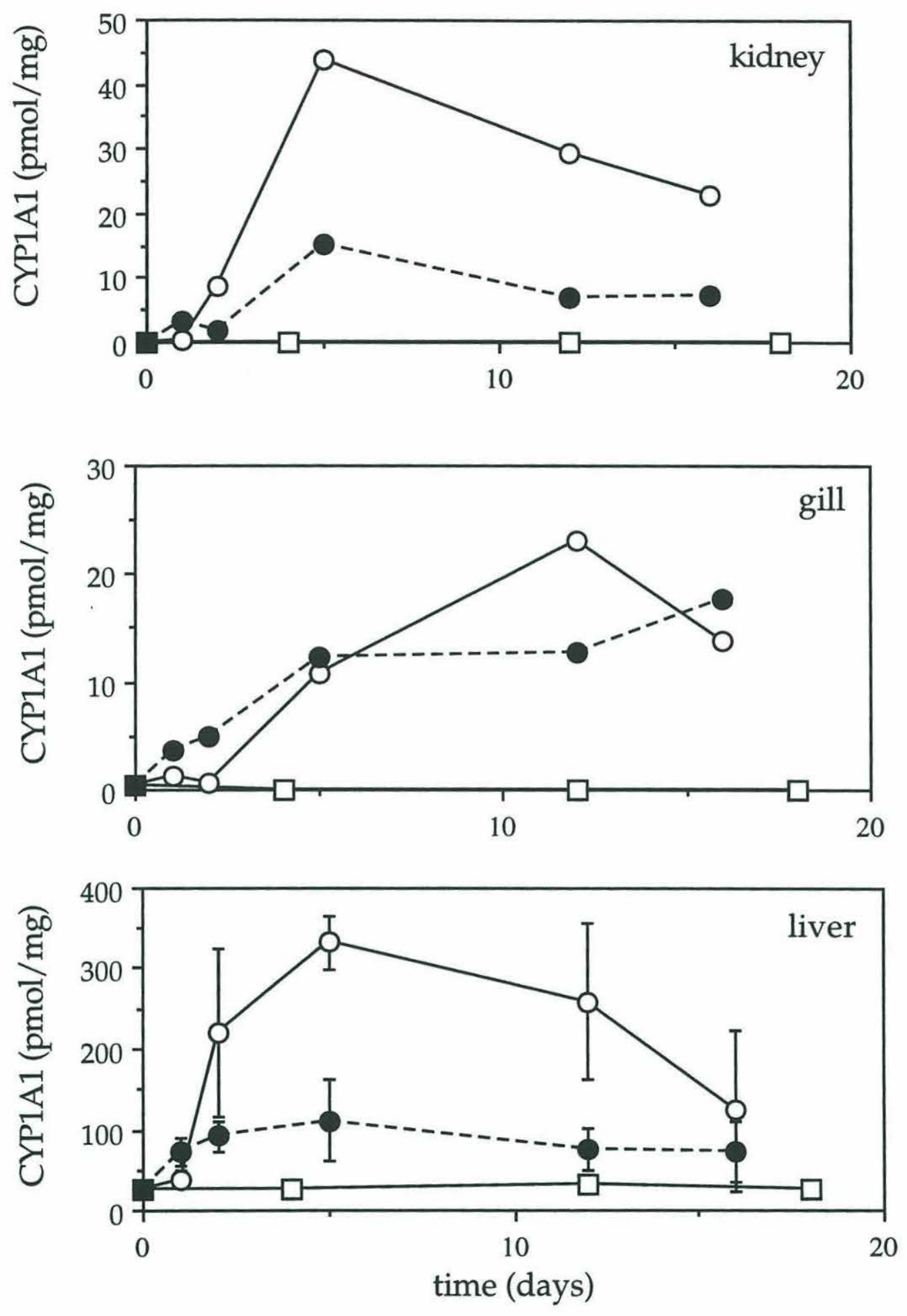
Fig 2. Rates of EROD activity in kidney, gill, and liver microsomes of TCB- and corn oiltreated scup. EROD activity was assayed fluorometrically, as described in Methods. Xaxis is the number of days subsequent to injection, $y$-axis is rate of activity in $\mathrm{pmol} / \mathrm{min} /$ mg microsomal protein. For kidney and gill, each point is the mean of two measurements, on a single pooled sample, composed of equally-sized tissue sections from 4 or 5 fish per treatment group. For liver, each point is the mean and standard deviation of measurements on 4 or 5 individuals per treatment group. Plot symbols are: square, corn oil control, open circle, $0.1 \mathrm{mg} / \mathrm{kg}$ TCB, closed circle, $5.0 \mathrm{mg} / \mathrm{kg}$ TCB. 

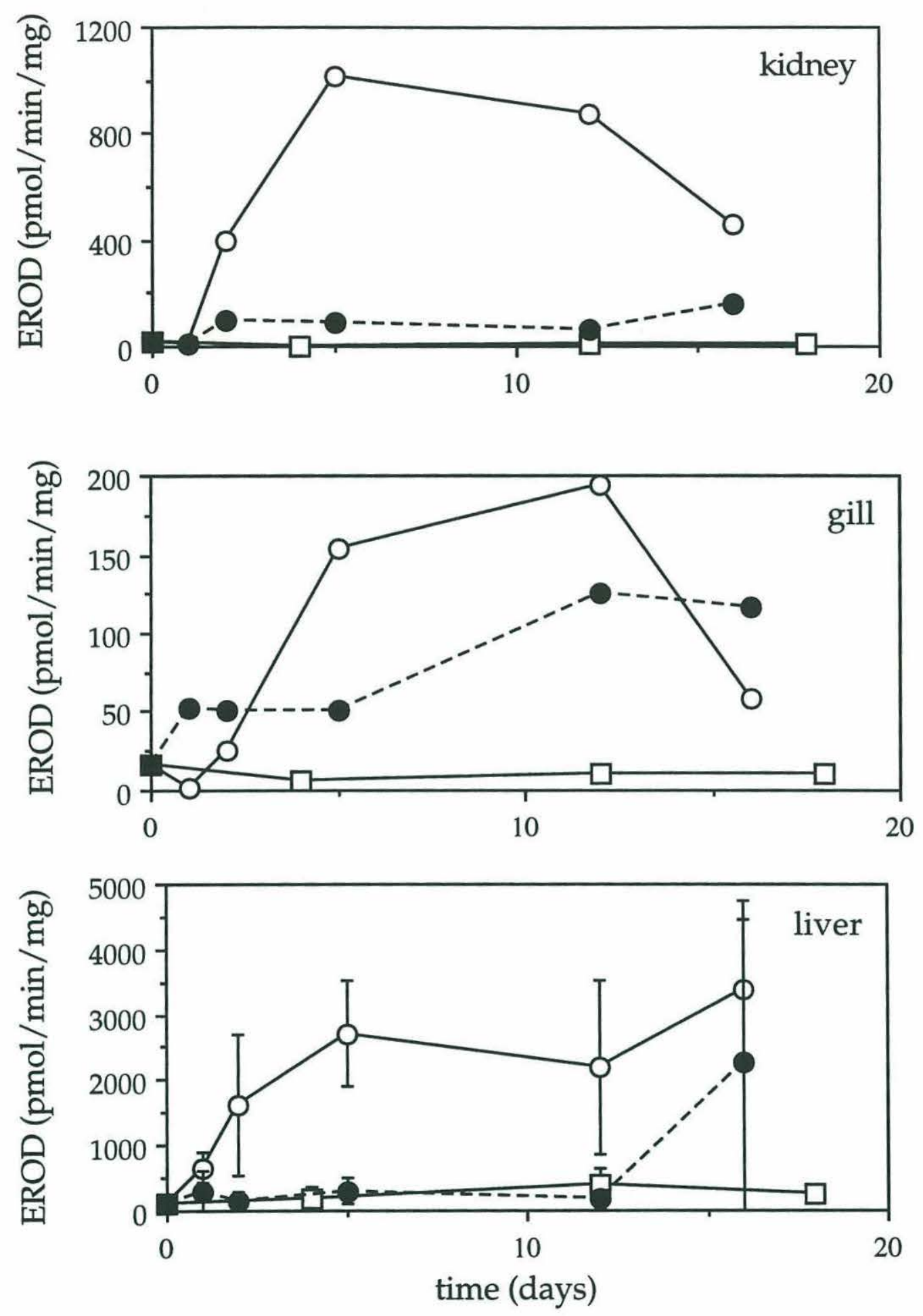
reduced EROD induction in gill (days 5 and 12), despite modest increases in CYP1A1 content in all organs at this dose. Suppressed EROD rates are consistent with competitive inhibition of EROD activity by residual TCB in microsomes (e.g. Gooch et al., 1989). EROD inhibition in the liver was released by day 16 in some fish treated with $5.0 \mathrm{mg} / \mathrm{kg}$ TCB, consistent with our finding that the bulk of the TCB was depurated from livers by this time point (chapter 2).

Although the absolute rates of EROD activity were far higher in liver than in extrahepatic organs of TCB-treated animals, both the specific activity (pmol EROD activity/ $\mathrm{min} / \mathrm{pmol} \mathrm{CYP} 1 \mathrm{~A} 1$ ) and the fold-induction were greater in the extrahepatics. For example, in the group of fish treated with $0.1 \mathrm{mg} / \mathrm{kg} \mathrm{TCB}$ and sampled on day 5 , specific activities were $8 \mathrm{pmol} / \mathrm{min} / \mathrm{pmol}$ for liver, 14 for gill, and 23 for kidney. In the same group of fish, there was a 15-fold induction of EROD activity in liver, a 28 -fold induction in gill, and a 232-fold induction in kidney, as compared to corn oil controls sampled on day 4.

\section{Immunohistochemistry}

CYP1A1 content was evaluated by scoring each cell type for intensity of CYP1A1-specific stain $(0-3)$ and for prevalence of CYP1A1-specific stain (0 - 3). Unless stated otherwise, cells from corn-oil treated fish did not stain specifically for CYP1A1. No cells stained in any of the control sections incubated with non-immune serum (mouse myeloma IgG) as a primary antibody.

Liver CYP1A1-specific stain was pervasive, occurring in all cells, in both the hepatocytes and the pancreatic tissue of all TCB-treated fish. Intensity of stain in the hepatocytes ranged from weak to strong in TCB-treated fish, and was significantly different between doses on day 5 (Figs $3 \mathrm{~B}$ and $3 \mathrm{C}$ ), at which time all low-dose fish received a score of 3 (strong) and all high-dose fish received scores of 1 or 2 (weak or moderate). Scores for hepatocyte induction are represented graphically in Fig 4A. Intensity of stain in the pancreas ranged from weak (days 1 - 5) to moderate (days 12 - 16), and stain scores did not differ between the two doses. CYP1A1-specific stain in sinusoidal endothelia was weak/ rare on day 1 and strong/ pervasive on days 12 and 16 at both doses (Fig 5A).

Kidney CYP1A1-specific stain was pervasive in renal tubule epithelia of most TCB-treated fish. Intensity of stain in the tubules was always heterogeneous, with populations of tubules variously staining weakly, moderately, or strongly. On days 2 through 16 , the percentage of renal tubules staining strongly was greater in fish treated with the low dose than in fish treated with the high dose of TCB, whereas the percentage of 
TABLE 1

CYP1A1 Induction in Renal Tubules of TCB-Treated Scup $a$

\begin{tabular}{c|ccc|ccc}
\hline Day & \multicolumn{3}{|c|}{$0.1 \mathrm{mg} / \mathrm{kg}$} & \multicolumn{3}{c}{$5.0 \mathrm{mg} / \mathrm{kg}$} \\
\hline & $\frac{\text { \% weak }}{100 b}$ & \% medium & \% strong & \% weak & \% medium & \% strong \\
1 & $100 b$ & & & \\
2 & $18 \pm 5^{c}$ & $50 \pm 14$ & $32 \pm 16$ & $37 \pm 36$ & $55 \pm 32$ & $9 \pm 9$ \\
5 & $10 \pm 6$ & $26 \pm 6$ & $64 \pm 8$ & $26 \pm 12$ & $39 \pm 14$ & $35 \pm 24$ \\
12 & $26 \pm 19$ & $27 \pm 5$ & $47 \pm 15$ & $26 \pm 6$ & $40 \pm 12$ & $34 \pm 14$ \\
16 & $24 \pm 10$ & $36 \pm 22$ & $40 \pm 23$ & $54 \pm 15$ & $27 \pm 14$ & $19 \pm 8$ \\
\hline
\end{tabular}

$a$ Kidney sections of TCB-treated fish were stained immunohistochemically for CYP1A1, as described in Methods. 100 renal tubules were counted from each individual kidney and assigned a score of either weak, medium, or strong for intensity of CYP1A1-specific staining.

$b$ On day one after treatment, tubules in each section stained homogeneously, and all tubules within each kidney section were scored as either weak or medium.

${ }^{c}$ Each measurement is a mean \pm standard deviation for 4 or 5 fish in each treatment group. 
Fig 3. Immunohistochemical analysis of CYP1A1 induction in liver and kidney. (A) Liver of fish treated with corn oil and sampled on day 4 (100x). Section showing no specific stain. (B) Liver of fish treated with $0.1 \mathrm{mg} / \mathrm{kg}$ TCB and sampled on day $5(100 \mathrm{x})$. Section showing strong/ pervasive stain in hepatocytes. (C) Liver of fish treated with $5.0 \mathrm{mg} / \mathrm{kg}$ TCB and sampled on day $5(100 x)$. Section showing moderate/ pervasive stain in hepatocytes, mild stain in pancreatic tissue (p). (D) Kidney of fish treated with corn oil and sampled on day 4 (100x). Section showing no specific stain in renal tubules (T), dark patches are melanomacrophage aggregates (m). (E) Kidney of fish treated with $0.1 \mathrm{mg} / \mathrm{kg}$ TCB and sampled on day $12(100 x)$. Section showing heterogeneous stain in renal tubules $(\mathrm{T})$, dark patches are melanomacrophage aggregates $(\mathrm{m})$. (F) Kidney of fish treated with $5.0 \mathrm{mg} / \mathrm{kg}$ TCB and sampled on day $12(100 \mathrm{x})$. Section showing heterogeneous stain in renal tubules $(\mathrm{T})$. 

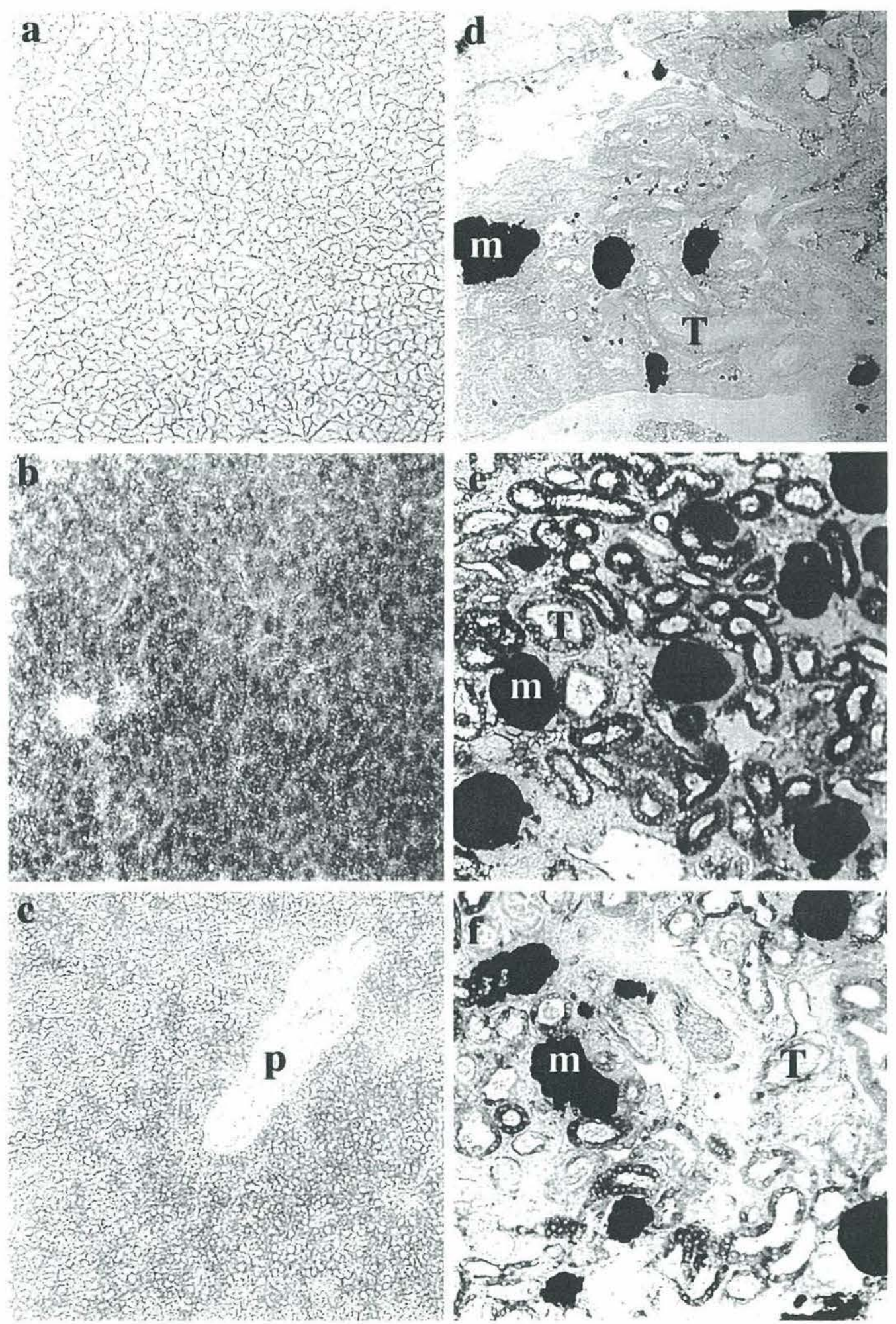

Sic m a t T

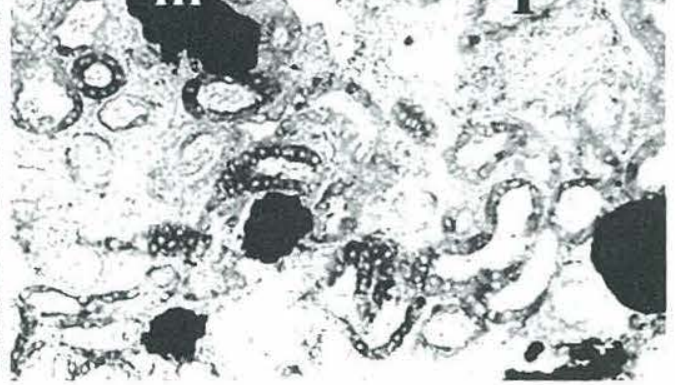


Fig 4. Time-course of CYP1A1 induction in immunostained epithelial cells. (A) Hepatocytes, (B) renal tubule epithelia, (C) kidney duct epithelia, (D) gill epithelia in nonproliferative regions, (E) mucosal epithelia of duodenum. $\mathrm{X}$-axis is the number of days subsequent to injection, $\mathrm{y}$-axis $(\mathrm{A}, \mathrm{C}, \mathrm{D}, \mathrm{E})$ is the staining score (intensity x prevalence), derived as described in Methods, or $\mathrm{y}$-axis (B) is the percentage of strongly staining renal tubules, determined as described in Table 1. Each point is the mean score for 4 or 5 fish per treatment group. Plot symbols are: square, corn oil control, open circle, $0.1 \mathrm{mg} / \mathrm{kg}$ TCB, closed circle, $5.0 \mathrm{mg} / \mathrm{kg}$ TCB. 
A: hepatocytes



B: kidney tubules

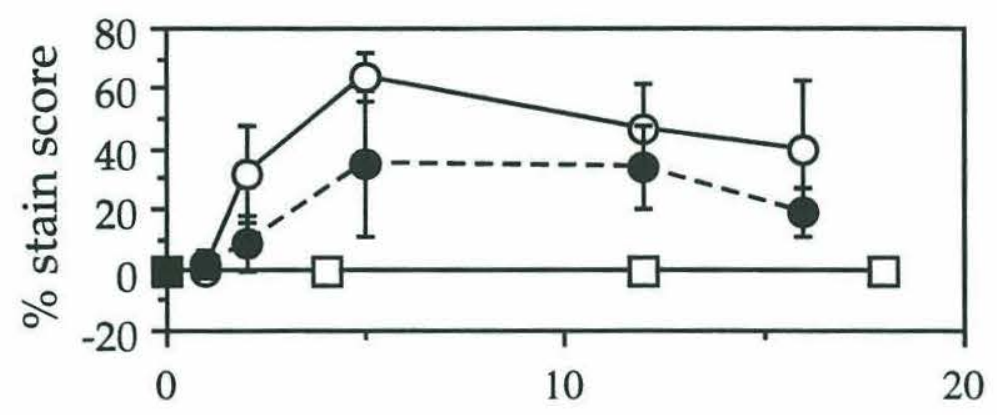

C: kidney ducts
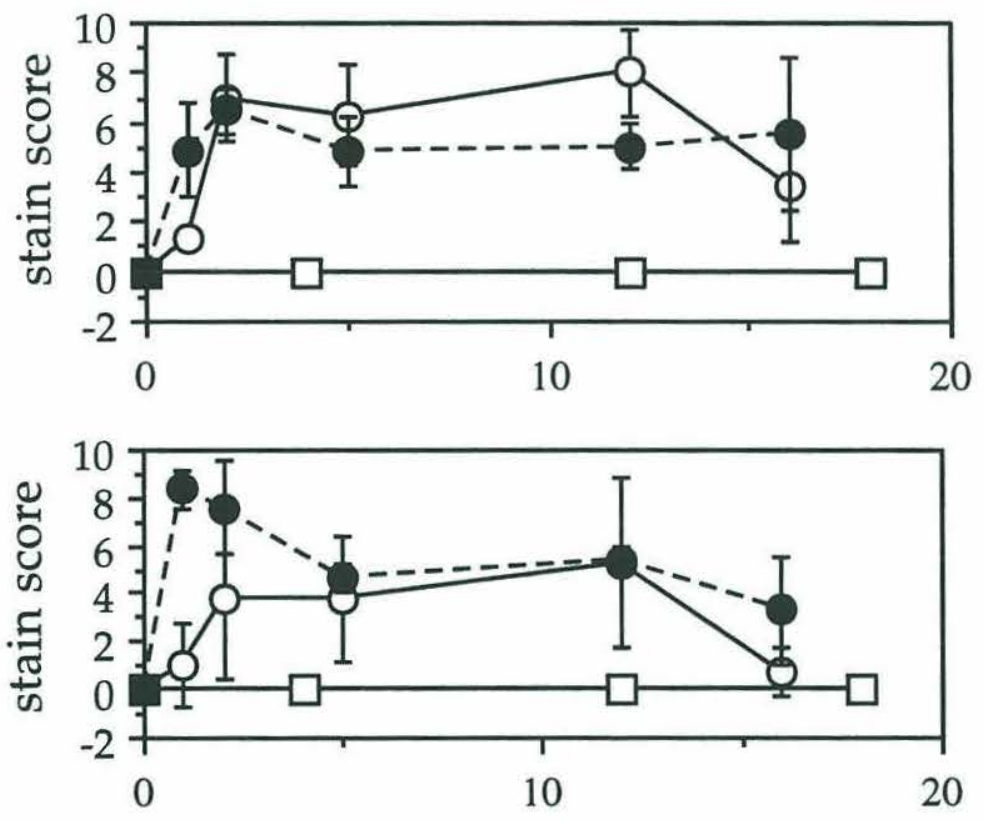

D: gill epithelia

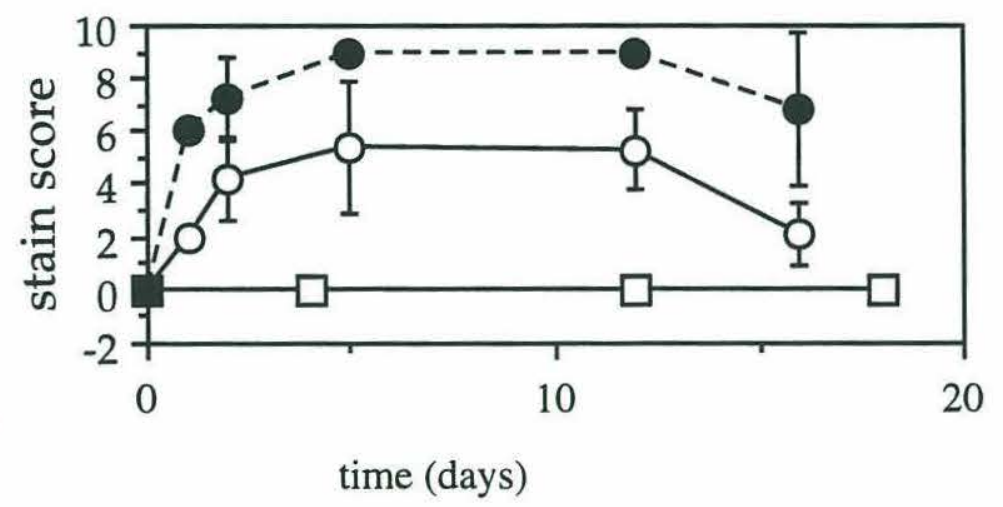


Fig 5. Time-course of CYP1A1 induction in immunostained endothelial cells. (A) Liver sinusoidal endothelia, (B) kidney vascular endothelia, (C) duodenum vascular endothelia, (D) atrial endocardia, (E) gonad vascular endothelia. $\mathrm{X}$-axis is the number of days subsequent to injection, $y$-axis (A, B, C, E) is the staining score (intensity $x$ prevalence), or $y$-axis (D) is the is the intensity of stain, derived as described in Methods. Each point is the mean score for 4 or 5 fish per treatment group. Plot symbols are: square, corn oil control, open circle, $0.1 \mathrm{mg} / \mathrm{kg} \mathrm{TCB}$, closed circle, $5.0 \mathrm{mg} / \mathrm{kg}$ TCB. 
A: liver sinusoids



B: kidney endothelia

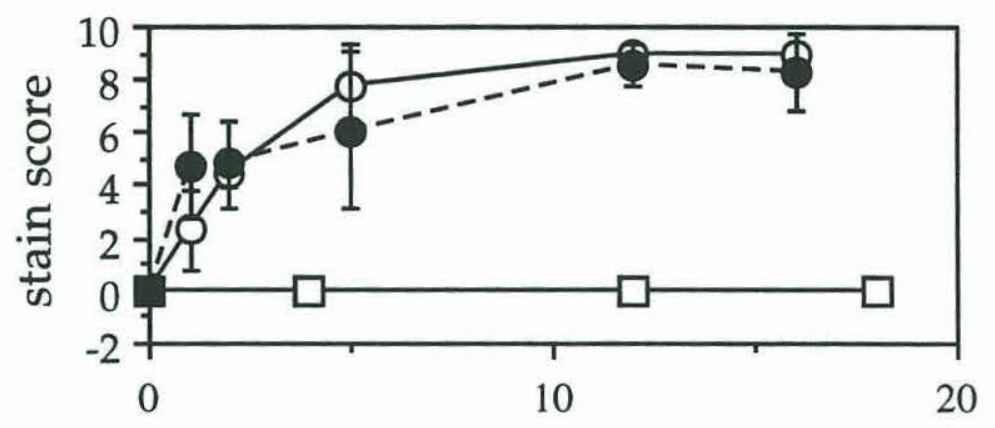

C: gut endothelia
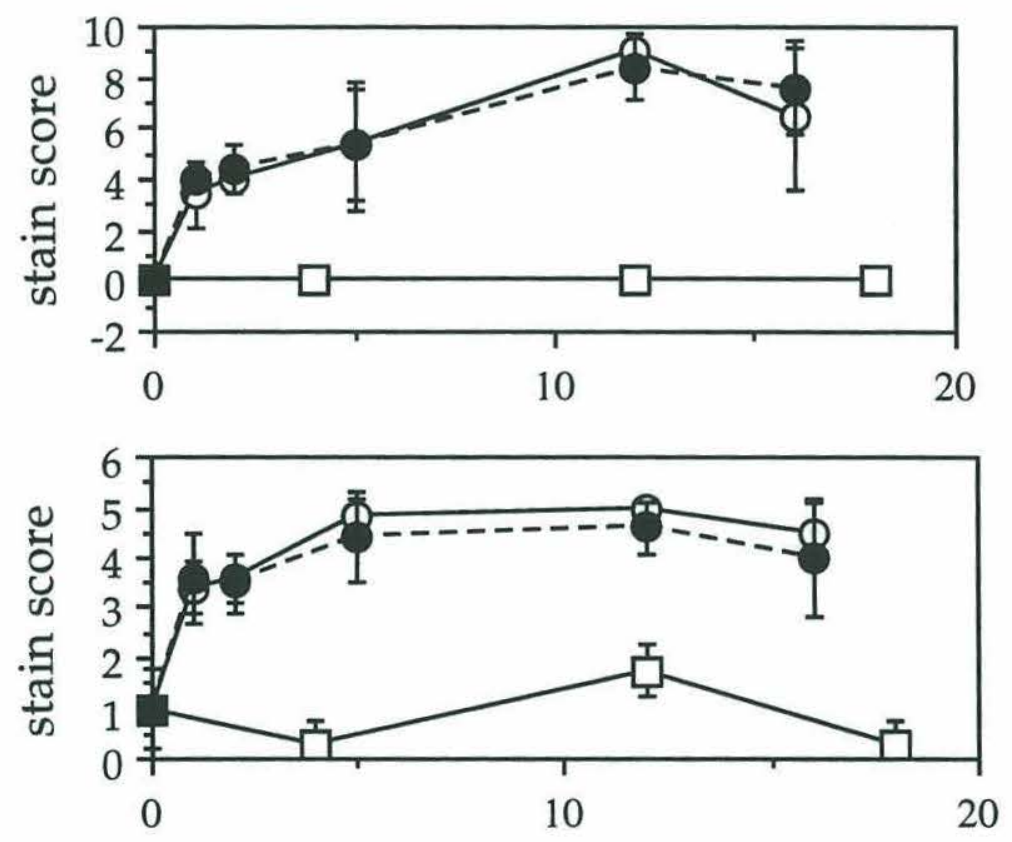

D: atrial endocardia

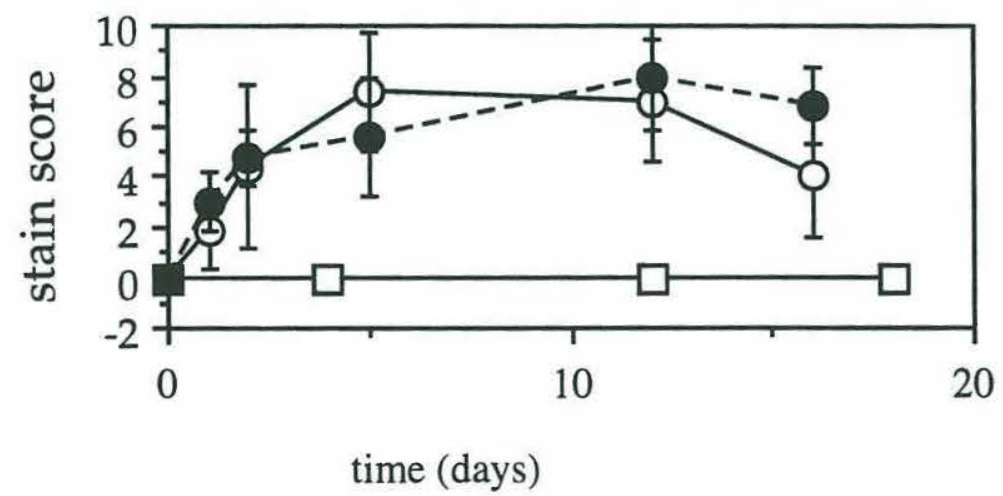


tubules staining weakly was greater in fish treated with the high dose (Table 1 and Figs $3 \mathrm{E}$ and $3 \mathrm{~F}$ ). Induction in renal collecting duct epithelia ranged from weak/ multifocal on day 1 to moderate/ pervasive on days 2 - 12, and was more intense on days 5 and 12 in the fish receiving the low TCB dose. Induction scores for kidney epithelia are presented graphically in Figs 4B and 4C. Induction in kidney vascular endothelium was similar to that in liver, with moderate/ multifocal stain by day 1, strong/ pervasive stain on days 12 and 16, and no dose-dependent differences in scores (Fig 5B).

Gill All corn oil-treated fish had heterogeneous/multifocal CYP1A1 staining in the gill pillar (endothelial) cells (Fig 6A), with some cells not staining and others staining weakly, moderately, or strongly. This is consistent with prior results (Miller et al., 1989) showing CYP1A1 staining in the pillar cells of depurated scup. In TCB-treated fish, pillar cell staining was pervasive (all or most cells stained) and heterogeneous at all time points (Figs 6B and 6D), and induction did not attenuate by day 16 .

Gill epithelial cells had a heterogeneous/ multifocal pattern of CYP1A1 induction in most TCB-treated fish, and did not stain in corn oil-treated fish. TCB treatment elicited a proliferation of epithelial cells between the secondary lamellae of gills (Fig 6D), which is described in a separate paper (chapter 4). Proliferation was evident by day 5, and was consistently more severe in fish treated with the higher TCB dose. The newly proliferative epithelia did not stain for CYP1A1, whereas the epithelial cells surrounding secondary lamellae in non-proliferative regions did stain for CYP1A1 (Figs 6B and 6D). Thus, overall CYP1A1 induction per unit tissue was greater at the low dose, which contained fewer proliferative (non-staining) cells. The pattern of CYP1A1 induction in the nonproliferative gill epithelia is presented graphically in Fig 4D. Prevalence of epithelial stain peaked on day 1 in fish treated with $5.0 \mathrm{mg} / \mathrm{kg}$, and declined concomitantly with the onset of cell proliferation (chapter 4).

There was a strong CYP1A1 induction in some TCB-treated fish in clusters of cells located at the base of each primary gill filament (Fig 6C). Examination of hematoxylin and eosin-stained slides suggests that these immunoreactive cells are chondrocytes. This cell type has previously been found to stain for CYP1A1 in environmentally exposed fish (Stegeman et al., unpublished results).

Intestine In fish treated with $0.1 \mathrm{mg} / \mathrm{kg}, \mathrm{CYP} 1 \mathrm{~A} 1$ induction generally occurred along the lumenal face of the mucosal epithelial cells (Fig 7B). In fish treated with 5.0 $\mathrm{mg} / \mathrm{kg}$, CYP1A1 induction was more intense and penetrated further into the mucosal epithelium, especially on days 5 and 12 (Fig 7C). At both doses induction in the mucosal epithelium was maximal on days 5 and 12 (Fig 4E), and the mean scores on day 16 were lower than those in fish sampled on day 12. The temporal pattern of CYP1A1 induction in 
vascular endothelium of intestine was similar to that in liver (Fig 5C), with moderate/ multifocal stain on day 1 , strong/ pervasive stain on day 12 , and no dose-dependent differences in scores. Neither the mean intensity of stain, nor the temporal pattern of induction, differed substantially between duodenal and caecal sections of intestine, in both epithelia and endothelia.

Spleen The vascular endothelium of major arterioles in spleen stained strongly and pervasively for CYP1A1 on days 12 and 16, at both TCB doses (Figs 7E and 7F). In addition, the capillary endothelium stained moderately and pervasively for CYP1A1 in all fish treated with $5.0 \mathrm{mg} / \mathrm{kg}$ and sampled on day 12 , in half of the fish treated with 5.0 $\mathrm{mg} / \mathrm{kg}$ and sampled on day 16 (2 of 4), but in only one fish of 8 treated with $0.1 \mathrm{mg} / \mathrm{kg}$ and from which spleen was sampled (Fig 7F).

Heart Endocardial cells of the atrium stained very weakly for CYP1A1 content (scoring 1 on a scale of $0-5$ ) in several of the corn oil-treated fish (Fig 8A). The intensity of CYP1A1 induction by TCB was stronger in atrial endocardia than in any other cell type examined in the organism, with scores of moderate to strong on day 1 (Fig 8C) and strong to very strong on days 5, 12, and 16 at both doses (Fig 5D). Ventricular endocardia stained very weakly to weakly on day 1 and moderately to strongly by day 12 , with no difference between doses. The endocardia of the bulbus arteriosus stained weakly on day 1 and strongly by day 5 at both doses (Fig 8B), in most animals staining more intensely than ventricle and less intensely than atrium. In several (70\%) of the fish treated with $5.0 \mathrm{mg} / \mathrm{kg}$ TCB and sampled on days 5,12 , and 16 , there was a weak immunoreactive stain in an unidentified cell type of the bulbus arteriosus, possibly capillary endothelia. This cell type also stained very weakly in two fish treated with $0.1 \mathrm{mg} / \mathrm{kg}$ TCB and sampled on days 5 and 12 , respectively.

Gonad Vascular endothelium was the only cell type that stained for CYP1A1 in gonad of both male and female fish exposed to TCB (Fig 8D). Staining ranged from moderate to strong and from multifocal to pervasive on days 5 and 12 (Fig 5E). There was no marked difference in induction between the sexes nor between the two TCB doses.

\section{Discussion}

In this study we show that the Ah receptor agonist TCB elicits a time-dependent induction of CYP1A1 in multiple organs of the marine fish scup, with intensity of induction in various cell types being either positively or negatively correlated with increasing dose of inducer. The results for day 5 are summarized in Table 2. In some cell types examined, the two TCB doses elicited a similar degree of induction, despite the 50- 
Fig 6. Immunohistochemical analysis of CYP1A1 induction in gill. (A) Gill of fish treated with corn oil and sampled on day $4(200 x)$. Section showing CYP1A1 stain in pillar cells (p), no stain in epithelia (e). (B) Gill of fish treated with $0.1 \mathrm{mg} / \mathrm{kg}$ TCB and sampled on day 12 (200x). Section showing heterogeneous CYP1A1 stain in pillar cells (p) and epithelial cells (e). (C) Gill of fish treated with $0.1 \mathrm{mg} / \mathrm{kg}$ TCB and sampled on day 2 $(1000 x)$. Section showing CYP1A1 stain in chondrocytes (all ovoid cells in figure). (D) Gill of fish treated with $5.0 \mathrm{mg} / \mathrm{kg}$ TCB and sampled on day $12(200 \mathrm{x})$. Section showing proliferation of epithelial cells between the secondary lamellae $(*)$, CYP1A1 stain in pillar cells all along the lamellae, and in epithelial cells in non-proliferative areas of lamellae. 

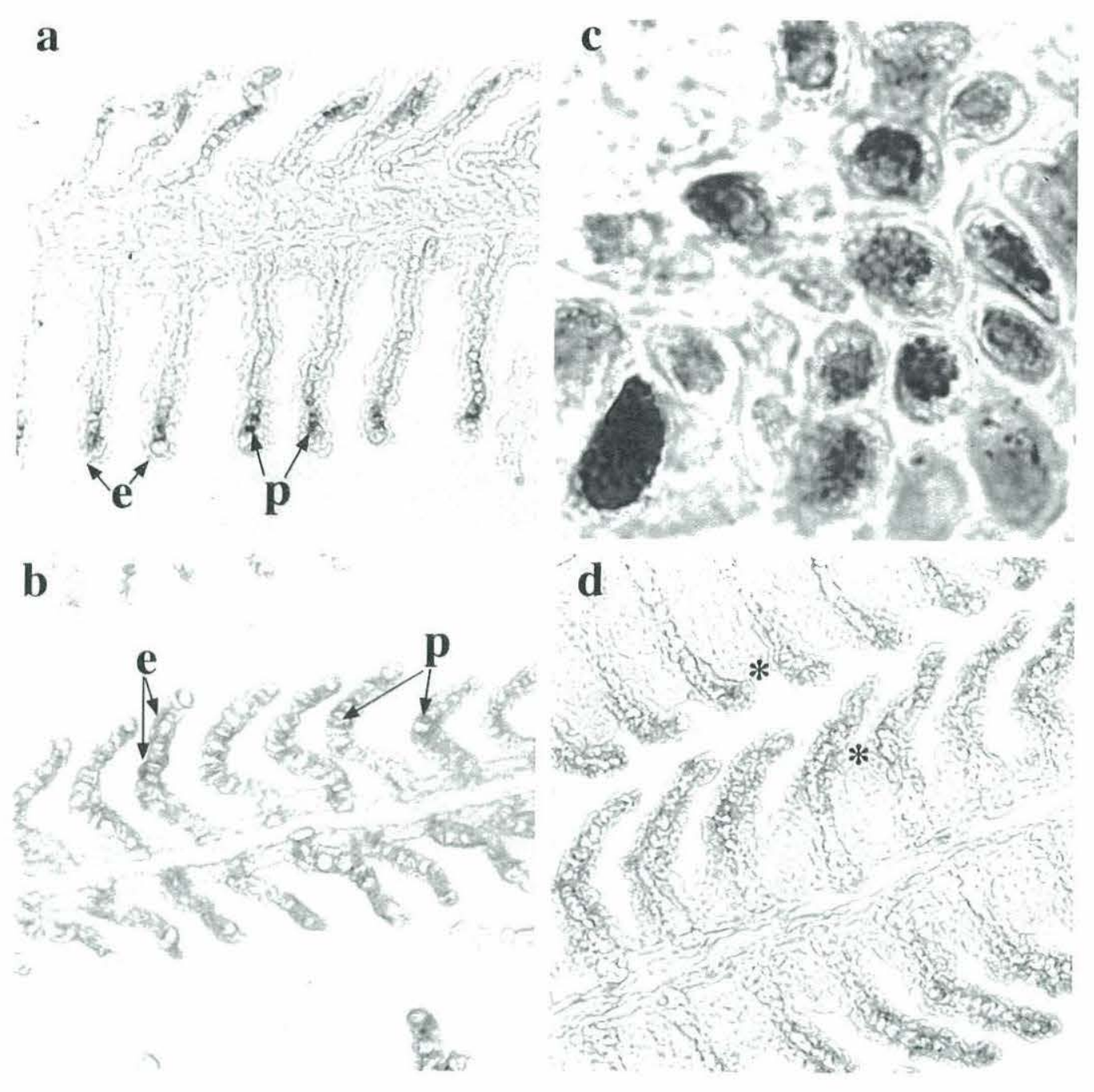
Fig 7. Immunohistochemical analysis of CYP1A1 induction in intestine and spleen. (A) Duodenum of fish treated with corn oil and sampled on day 4 (100x). Section showing no specific stain, L indicates lumen of intestine. (B) Caecum of fish treated with $0.1 \mathrm{mg} / \mathrm{kg}$ TCB and sampled on day $5(100 x)$. Section showing moderate stain along lumenal edge of mucosal epithelia, indicated are lumen (L) and goblet cells (g). (C) Caecum of fish treated with $5.0 \mathrm{mg} / \mathrm{kg}$ TCB and sampled on day $12(100 \mathrm{x})$. Section showing strong/ pervasive stain in mucosal epithelia, indicated are lumen (L) and goblet cells (g). (D) Spleen of fish treated with corn oil and sampled on day $16(100 x)$. Section showing no specific stain, dark patches are melanomacrophage aggregates $(\mathrm{m})$. (E) Spleen of fish treated with 0.1 $\mathrm{mg} / \mathrm{kg}$ TCB and sampled on day 12 (100x). Section showing strong CYP1A1 stain in vascular endothelia of major arterioles (A), dark patches are melanomacrophage aggregates (m). (F) Spleen of fish treated with $5.0 \mathrm{mg} / \mathrm{kg}$ TCB and sampled on day 16 . Section showing strong CYP1A1 stain in vascular endothelia of major arterioles (A), moderate stain in vascular endothelia of sinusoids (s), dark patches are melanomacrophage aggregates $(\mathrm{m})$. 

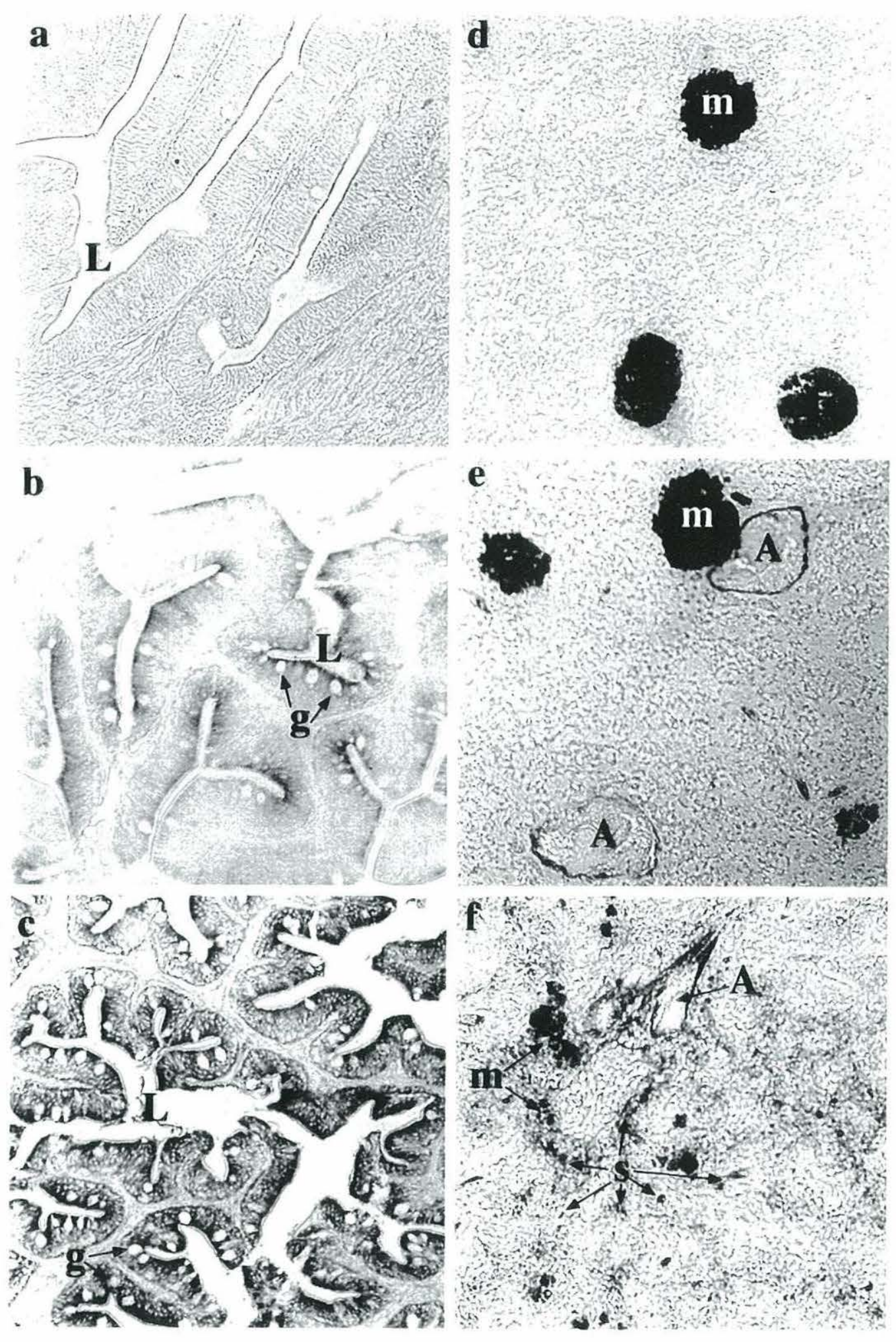
Fig 8. Immunohistochemical analysis of CYP1A1 induction in heart and gonad. (A) Heart of fish treated with corn oil and sampled on day $12(100 x)$. Section showing very weak CYP1A1 stain in endocardia of atrium (a), no stain in ventricle (v). (B) Bulbus arteriosus (heart) of fish treated with $5.0 \mathrm{mg} / \mathrm{kg}$ TCB and sampled on day 16 (100x). Section showing very strong stain in vascular endothelia, weak stain in capillary endothelia. (C) Heart of fish treated with $5.0 \mathrm{mg} / \mathrm{kg}$ TCB and sampled on day 1 (100x). Section showing strong CYP1A1 stain in atrial endocardia (a), very weak stain in ventricular endocardia (v). (D) Representative gonad sections from TCB treated fish. Top: ovary of fish treated with $5.0 \mathrm{mg} / \mathrm{kg}$ TCB and sampled on day 12 , bottom: testis of fish treated with $0.1 \mathrm{mg} / \mathrm{kg} \mathrm{TCB}$ and sampled on day 5. Sections showing strong/pervasive stain in vascular endothelia (e). 

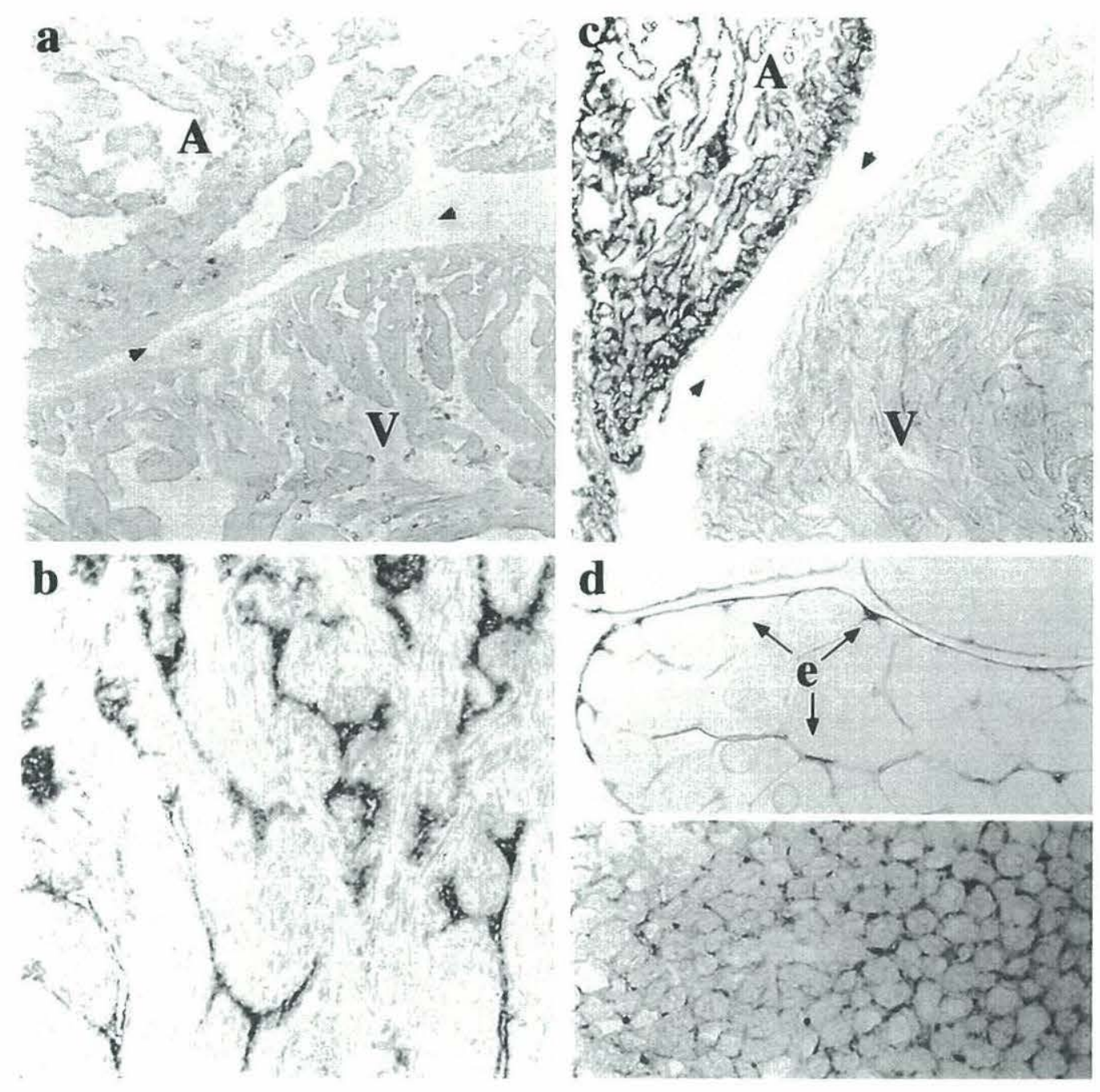
TABLE 2

CYP1A1 Induction in Multiple Cell Types of TCB-Treated Fish on Day 5

\begin{tabular}{|c|c|c|c|c|}
\hline & \multicolumn{2}{|c|}{$0.1 \mathrm{mg} / \mathrm{kg}$ TCB } & \multicolumn{2}{|c|}{$5.0 \mathrm{mg} / \mathrm{kg}$ TCB } \\
\hline Cell Type & Intensity ${ }^{a}$ & Prevalence $b$ & Intensity & Prevalence \\
\hline$\underline{\text { Liver }}$ & & & & \\
\hline hepatocyte & +++ & +++ & $+/++$ & +++ \\
\hline pancreatic epithelium & + & +++ & + & +++ \\
\hline sinusoidal endothelium & +++ & ++ & ++ & ++ \\
\hline$\underline{\text { Kidney }}$ & & & & \\
\hline renal tubule & $++/+++$ & +++ & $+/++/+++$ & +++ \\
\hline renal duct & $+/++$ & +++ & $+/++$ & +++ \\
\hline vascular endothelium & +++ & +++ & $++/+++$ & +++ \\
\hline$\underline{\text { Gill }}$ & & & & \\
\hline epithelium (non-proliferative) & $++/+++$ & $+/++$ & $++/+++$ & ++ \\
\hline pillar endothelium & $++/+++$ & +++ & $++/+++$ & +++ \\
\hline$\underline{\text { Intestine }}$ & & & & \\
\hline duodenal mucosa & $+/++$ & +++ & +++ & +++ \\
\hline caecal mucosa & ++ & +++ & $++/+++$ & +++ \\
\hline vascular endothelium & $++/+++$ & ++ & $++/+++$ & ++ \\
\hline Spleen (day 12) ${ }^{c}$ & & & & \\
\hline arteriole endothelium & +++ & +++ & +++ & +++ \\
\hline capillary endothelium & - & - & ++ & +++ \\
\hline$\underline{\text { Heart }}$ & & & & \\
\hline atrial endocardium & +++ & +++ & $++/+++$ & +++ \\
\hline ventricular endocardium & ++ & +++ & ++ & +++ \\
\hline bulbus endocardium & $++/+++$ & +++ & $++/+++$ & +++ \\
\hline bulbus capillary endothelium & - & - & $-1+$ & ++ \\
\hline$\underline{\text { Gonad }}$ & & & & \\
\hline ovary endothelium & $++/+++$ & $++/+++$ & $++/+++$ & $++/+++$ \\
\hline testis endothelium & +++ & +++ & $++/+++$ & $++/+++$ \\
\hline
\end{tabular}

$a$ Intensity is the density of peroxidase stain ( $+=$ weak, $++=$ moderate, $+++=$ strong)

$b$ Prevalence is the frequency of stained cells $(+=$ rare,$++=$ multifocal, $+++=$ pervasive)

$c$ Spleen was not sampled on day 5 , so day 12 scores are shown

Note: in vehicle-treated fish, the only cell types staining for CYP1A1 were gill pillar endothelium and atrial endocardium, as described in Results. 
fold difference in dose: pancreatic tissue of liver, gill pillar endothelium, endocardium of the major vessels in all three heart chambers, and vascular endothelium of intestine, spleen (major arterioles), and gonad. In a few cell types, induction was stronger (either more intense or more prevalent) in fish treated with the high TCB dose: gill epithelia (day 1), intestinal mucosa, and capillary endothelia of spleen and bulbus arteriosus. Interestingly, induction was stronger in some epithelial cell types in the fish treated with the low TCB dose: hepatocytes (day 5), renal collecting ducts and tubules, and total gill epithelia (the mean of proliferative and non-proliferative cells).

In every organ of each fish examined, induction was seen in one or more cell types within 24 hours of treatment. CYP1A1 content reached a peak of prevalence and intensity on day 5 in the following epithelia: hepatocytes, renal tubules, and intestinal mucosa. In contrast, the CYP1A1 stain in the vascular endothelium of these three organs reached a peak of prevalence and intensity on day 12. In several epithelial cell types, there was the suggestion of attenuation of CYP1A1 signal by day 16, particularly in fish treated with 0.1 $\mathrm{mg} / \mathrm{kg}$ TCB (Fig 4). This is consistent with our finding (chapter 2) that hepatic TCB residues decline in these fish by day 16 . By contrast, CYP1A1 induction in the endothelium of most organs did not decline by day 16 at either dose. In fact, CYP1A1 induction remained moderate to strong in vascular endothelium of every organ examined in a single fish that was treated with $0.1 \mathrm{mg} / \mathrm{kg}$ TCB and killed on day 34 after treatment (data not shown). These findings are consistent with those of Hahn and Stegeman (1994), who found sustained CYP1A1 induction in microsomal preparations of heart and gill from scup treated with the Ah receptor agonist 2,3,7,8-tetrachlorodibenzofuran. The sustained induction in the latter study may have been due largely to an induction in endothelial cells of heart and gill.

Vascular endothelium CYP1A1 was induced in vascular endothelium of every organ examined, and in some tissues (spleen, heart, gonad) this was the only cell type showing CYP1A1 induction, consistent with earlier observations in scup (Smolowitz et al., 1991). With intraperitoneal treatment, TCB may be absorbed directly into the bloodstream without first passing through the liver or intestine for possible metabolism. Rapid bloodmediated distribution of TCB is indicated by moderate CYP1A1 induction in vascular endothelium of organs throughout the body, within 24 hours of TCB treatment.

CYP1A1 induction in vascular endothelium and endocardium generally did not differ between the two TCB doses. In some organs (liver, kidney, gonad), the mean score for CYP1A1 staining on day 5 was greater at the low dose than at the high dose (Table 2), but in each case the induction increased between days 5 and 12 (Fig 5), such that equal, strong endothelial induction was seen on day 12 in all organs. Equivalent induction by the 
two doses may suggest that the low dose was sufficient to elicit maximal CYP1A1 induction in this cell type. Conversely, the equivalent induction suggests that the high dose did not cause a reduced CYP1A1 induction in this cell type, as it did in some epithelia (discussed below). Alternatively, it is possible that our staining and scoring methods cannot discern subtle differences in degree of induction in these small cells. In studies with cultured porcine aorta endothelial cells, degree of CYP1A1 induction has been found to be directly correlated with TCB dose, with the exception of cells treated with very high doses (Stegeman et al., in press), demonstrating that vascular endothelia can express greater CYP1A1 in response to greater inducer concentration. A dose-response study with fish endothelial cells is needed.

Gonad Vascular endothelium was the only cell type showing CYP1A1 induction in either male or female gonad. Even toxic TCB doses $(5.0 \mathrm{mg} / \mathrm{kg})$ still did not elicit CYP1A1 induction in germinal cells. Similarly, in female fathead minnow exposed to TCB via the water, endothelium was the only cell type that stained for CYP1A1 in ovary, despite appreciable TCB accumulation in this organ (Lindstrom-Seppa et al., 1994). In an earlier study of TCB-treated scup (Smolowitz et al., 1991), weak induction was noted in ovigerous lamellar epithelium and spermatic duct epithelium, but no induction was seen in eggs or sperm.

Heart As in the present study, other recent investigations of fish treated with CYP1A1 inducers have found stronger CYP1A1 induction in atrial endocardium than in endocardial cells of ventricle or bulbus arteriosus (Smolowitz et al., 1991; Stegeman et al., 1991). This is the first report of CYP1A1 induction in a cell type of the bulbus arteriosus that we have tentatively identified as capillary endothelium. Induction in this cell type was seen weakly in many fish exposed to $5.0 \mathrm{mg} / \mathrm{kg}$, and very weakly in two fish exposed to $0.1 \mathrm{mg} / \mathrm{kg}$ TCB. A similar pattern of induction to that in bulbus arteriosus was seen in spleen endothelium, and is discussed below.

Spleen CYP1A1 was strongly induced in the vascular endothelium of the major arterioles of spleen at both TCB doses. Additionally, there was a distinct, though moderate, induction in the capillary endothelium of many fish treated with $5.0 \mathrm{mg} / \mathrm{kg} \mathrm{TCB}$. This is consistent with a greater penetration of TCB into the organ at higher doses. We speculate that at lower TCB doses, CYP1A1 in endothelia of major arterioles may be capable of binding and sequestering a large fraction of the TCB, thereby preventing downstream flow of the compound. A similar sequestering function has been postulated for CYP1A2 binding of TCDD in mouse liver (Poland et al., 1989). At higher TCB doses, however, a greater concentration of TCB in blood may exceed the endothelial cells' "capacity" for sequestration, allowing CYP1A1 induction by TCB in downstream cells. 
The capillary endothelia in the present study consistently stained more weakly than did the arteriole endothelia, possibly indicating that a lower concentration of inducer was reaching the capillaries, and consistent with inducer sequestration in the arterioles. This finding further supports our suggestion (Stegeman et al., 1989; Smolowitz et al., 1991) that vascular endothelium may serve as a first site of defense against blood-borne xenobiotics in many vertebrate organs. The hypothesis regarding inducer sequestration is currently being tested in teleost endothelial cells.

Intestine CYP1A1 induction in mucosal epithelia occurred primarily along the lumenal edge of cells, the edge that comes into contact with gut contents, in fish treated with $0.1 \mathrm{mg} / \mathrm{kg}$. In fish treated with $5.0 \mathrm{mg} / \mathrm{kg}$, induction in mucosal epithelia was more intense at the lumenal edge and penetrated further into the cells from the lumenal face. Studies with mice and rats indicate that a substantial fraction of administered TCB is excreted in feces, both as parent compound and as metabolites (Yoshimura et al., 1987; Klasson Wehler et al., 1989), thus necessitating passage through the intestine. In fish too, $\mathrm{PCB}$ has been found to be excreted as parent compound in bile (Stein et al., 1984). We report the presence of both TCB and TCB metabolites in the bile of these scup (White et al., in preparation). Bile residue data indicate that metabolite production was suppressed in fish administered $5.0 \mathrm{mg} / \mathrm{kg}$ TCB , as compared to those treated with $0.1 \mathrm{mg} / \mathrm{kg}$, possibly due to lower hepatic CYP1A1 content in the high-dose group. Thus fish treated with the low dose had appreciable quantities of TCB metabolites and very little parent TCB in bile, whereas those treated with the high dose had small amounts of metabolites and very high concentrations of parent TCB in bile (White et al., in preparation). Given the lumenal induction in mucosal epithelia and given the TCB in bile, we assume a biliary route of exposure of intestinal mucosa to TCB. The bile residue data imply that mucosal epithelia in fish treated with $5.0 \mathrm{mg} / \mathrm{kg}$ were exposed to far higher concentrations of TCB than were the mucosal epithelia in fish treated with $0.1 \mathrm{mg} / \mathrm{kg}$, explaining the substantial difference in degree of CYP1A1 induction between doses. The intensity of CYP1A1 induction was similar in duodenal and caecal sections, both of which are located near the site where the bile duct drains into the intestine in scup.

Gill CYP1A1 induction in both gill pillar (endothelial) cells and gill epithelial cells was heterogeneous at both TCB doses, with cells of each type variously staining weakly, moderately, or strongly. In the present study the fish were held in a constant flow-through tank, thus TCB was unlikely to accumulate in the water, and gill was likely exposed via the bloodstream. Among the fish treated with $0.1 \mathrm{mg} / \mathrm{kg}$ TCB, CYP1A1 content in gill microsomes peaked at a later time point than did CYP1A1 in liver and kidney microsomes (Fig 1), indicating that TCB content may have peaked later in gill. This is in agreement 
with prior studies showing a much slower apparent elimination of PCB from gill than from any other organ of treated fish (Guiney et al., 1977; O'Connor and Pizza, 1987), and is consistent with gill being a major site of PCB egress from the organism, as has been suggested previously (O'Connor and Pizza, 1987; Miller et al., 1989).

TCB elicited a proliferation of epithelial cells between the secondary lamellae of gill, which was particularly pronounced in fish treated with $5.0 \mathrm{mg} / \mathrm{kg}$. The newly formed epithelial cells did not stain for CYP1A1; epithelial cell induction was observed only on secondary lamellae without proliferation, or at the tips of secondary lamellae that had proliferating cells at the base (Fig 6D). Thus microsomal CYP1A1 in gill was greater at the low dose than at the high dose, since substantially less gill tissue was composed of proliferating epithelia at the low dose. Our results are consistent with a growing body of evidence suggesting that Ah receptor acts differently in proliferating and differentiating cells, often showing a lower responsiveness in actively proliferating epithelia (e.g. Traber et al., 1992). In the present study, administration of an Ah receptor agonist apparently affects cell proliferation, and such proliferation in turn seems to affect Ah receptor action.

Kidney CYP1A1 induction in renal tubules was heterogeneous at both TCB doses, with whole tubule cross-sections staining either weakly, moderately, or strongly for CYP1A1 content. Heterogeneity of induction in renal tubule epithelia has been noted previously in studies of fish exposed to Ah receptor agonists (Smolowitz et al., 1991; Smolowitz et al., 1992), but has not previously been described quantitatively. In fish treated with the high dose, a smaller percentage of the tubules stained strongly, and a greater percentage stained weakly, than in fish treated with the low dose (Table 1). Thus microsomal CYP1A1 content, a measure of mean expression across the tissue, was greater at the low dose.

In a separate study describing the histopathology of these fish (chapter 4), we report that TCB treatment elicited degeneration and necrosis of renal tubules. By day 12 , TCB-treated kidneys displayed chronic necrosis mixed with regeneration, indicated by the presence of both necrotic tubular epithelia and new, cuboidal epithelia. Fish treated with the high dose displayed more cell necrosis, and hence more generation of new tissue, than did fish treated with the low dose. The weakly immunostaining foci in kidney sections from high-dose fish included both strongly necrotic tubules and seemingly healthy, regenerated tubules. We therefore speculate that the weaker overall induction in renal epithelia at the high dose may have been related to tissue pathology: severely necrotic cells and newly formed cells may generally express less CYP1A1 than do older, healthier cells.

Liver In the livers of animals sampled on day 5, CYP1A1 staining in hepatocytes was strong/ pervasive in those treated with $0.1 \mathrm{mg} / \mathrm{kg}$, and was weak to moderate/ 
pervasive in those treated with $5.0 \mathrm{mg} / \mathrm{kg}$. Thus the "reduced" induction in the liver of fish treated with the high TCB dose was qualitatively different from that in kidney and gill. Whereas the kidney and gill of fish treated with $5.0 \mathrm{mg} / \mathrm{kg}$ had a reduced percentage of strongly staining epithelial cells, the liver of these fish had weaker CYP1A1 staining in most if not all hepatocytes. A difference between doses was only apparent on day 5 (Fig $4 \mathrm{~A}$ ); by day 12 the induction remained at a moderate level in the high-dose fish, and had already begun to decline in the low-dose fish. These results are confirmed by immunoblot data (Fig 1), which indicate a sizable difference in hepatic CYP1A1 content between doses on day 5 , followed by a decline in CYP1A1 content at the low dose and a consequent closing of the "gap" between doses.

In sinusoidal endothelium, by contrast, CYP1A1 was generally induced to the same degree by both TCB doses. Although mean endothelial induction was stronger on day 5 at the low dose than at the high dose, induction increased between days 5 and 12 at the high dose (unlike in the hepatocytes), such that strong and pervasive induction occurred on day 12 at both doses. The difference in time-course for endothelial induction by the two doses may be due to differences in TCB disposition at the two doses: we found that hepatic TCB concentration peaked on day 5 at the low dose, and on day 12 at the high dose (chapter 2). The "suppressed" induction in liver epithelia, but not endothelia, may be due to greater TCB concentration in epithelia than in endothelia, as has been found in studies of rat treated with TCB by intraperitoneal injection (Durham and Brouwer, 1989). In addition, differences between epithelial and endothelial CYP1A1 content may be related to different rates of substrate turnover by CYP1A1 in the two cell types (see below).

Although CYP1A1 was induced to an equal extent by the two TCB doses in endothelial and pancreatic cells of the liver, total microsomal CYP1A1 was significantly higher in livers of fish receiving the low TCB dose. Morphometric analysis of trout liver indicates that approximately $80 \%$ of the liver volume is occupied by hepatocytes (Hampton et al., 1989). Thus differences in CYP1A1 expression in hepatocytes largely shape the profile seen in liver microsomes.

Further analysis of hepatic microsomes from these scup (chapter 2) indicated that CYP1A1 was the only enzyme with strongly reduced content in animals treated with 5.0 $\mathrm{mg} / \mathrm{kg}$ TCB. All other enzymes examined (cytochrome b5, NADPH cytochrome c reductase, UDP glucuronosyl transferase, the putative scup CYP2B, and the putative scup CYP3A) were expressed at nearly equal levels in livers of fish treated with $0.1 \mathrm{mg} / \mathrm{kg}$ and $5.0 \mathrm{mg} / \mathrm{kg}$. Furthermore, in vitro studies in liver (chapter 2) indicate that TCB occupation of the CYP1A1 active site promotes the inactivation of CYP1A1 in the presence of $\mathrm{NADPH}$, which appears to involve the production of $\mathrm{H}_{2} \mathrm{O}_{2}$ or other reactive oxygen 
species in the active site. We speculate that a similar mechanism may cause CYP1A1 inactivation and degradation in vivo, and thus may account for the reduced content of immunodetectable CYP1A1 in hepatic microsomes (Fig 1) and liver sections (Fig 3C) of fish treated with a high TCB dose.

Whether TCB elicits the production of reactive oxygen in other tissues, and the resultant effects, are not now known. It is possible that reactive oxygen is partially responsible for the pathology seen in gill and kidney, and for the reduced CYP1A1 content in gill and kidney epithelia of fish treated with $5.0 \mathrm{mg} / \mathrm{kg}$ TCB. It is noteworthy that "suppressed" CYP1A1 content at the high TCB dose was only seen in epithelial cells; endothelia of all organs examined had equally strong CYP1A1 induction at the two doses. Prior investigations (Stegeman et al., 1982; Stegeman et al., 1989) indicate that the ratio of NADPH cytochrome $\mathrm{c}$ reductase : cytochrome P450 is ten-fold lower in scup heart (where all of the P450 is endothelial) than in scup liver (where most of the P450 is epithelial). Limiting quantities of reductase enzyme in endothelia may be expected to result in lower P450 catalytic activity in these cells, even with highly induced P450 content. If the rates of electron transport and substrate turnover are lower for endothelial CYP1A1 than for epithelial CYP1A1, the endothelia may have a lower potential to produce reactive oxygen species in the presence of TCB, which appears to be linked to suppressed enzyme content. It is possible that reactive oxygen species also contribute to the cell proliferation that has been observed in several organs of these TCB-treated scup (Moore et al., in preparation). All of these questions will warrant further examination, with both in vitro and in vivo studies.

In summary, dose-response patterns for CYP1A1 induction were found to differ substantially between cell types, with epithelia displaying a broader range of response than endothelia. The pervasiveness and persistence of CYP1A1 in endothelium provides further evidence of the potential significance of CYP1A1 induction in this cell layer of vertebrate organs, and is particularly noteworthy in light of the finding that endothelium is the most common site of induction in fish that have been chronically exposed in the wild (Stegeman et al., 1991). Of the epithelia examined, intestinal mucosa responded to the higher dose with stronger induction, showing no signs of toxicity. Epithelial cells of liver, kidney, and gill showed lower overall induction in response to the higher TCB dose. Several factors may contribute to this unexpected response, including Ah receptor-mediated toxicity to epithelial cells, toxicity caused by the in situ production of reactive oxygen species, and oxidative inactivation of CYP1A1 due to active-site binding by TCB. 


\section{CHAPTER FOUR}

Histopathological Analysis of 3,3',4,4'-Tetrachlorobiphenyl

Toxicity in Multiple Organs of the Marine Fish Scup:

a Time-Course and Dose-Response Study 


\section{Abstract}

Coplanar polychlorinated biphenyl (PCB) congeners elicit a suite of toxic effects in mammals, similar to those seen following treatment with 2,3,7,8-tetrachlorodibenzo-pdioxin, that are believed to be mediated by binding to the cytosolic Ah receptor. Although PCBs are widespread contaminants in marine environments, very little work has addressed the histopathology of fish treated with PCB, particularly in extrahepatic organs. In the present study the marine fish scup (Stenotomus chrysops) was treated with the Ah receptor agonist 3,3',4,4'-tetrachlorobiphenyl (TCB) by a single intraperitoneal injection at two doses, $0.1 \mathrm{mg} / \mathrm{kg}$ and $5.0 \mathrm{mg} / \mathrm{kg}$. At time points from 1 to 16 days after treatment, groups of animals were killed and several tissues were fixed and stained for examination under light microscopy. Residue analysis indicated that hepatic TCB concentration was maximal on day 5 in fish administered $0.1 \mathrm{mg} / \mathrm{kg}$, and on day 12 in fish administered $5.0 \mathrm{mg} / \mathrm{kg}$, followed by a decline in TCB content at both doses. In all TCB-treated fish there was an increased basophilia of hepatocytes, suggesting proliferation of endoplasmic reticulum and a loss of lipid and glycogen stores. Hepatocyte nuclei were enlarged but regular in some TCB-treated fish. TCB-treated fish had a greater number of melanomacrophage aggregates in spleen than did vehicle-treated fish. In kidney, TCB treatment induced a dilation (day 5) and eventual necrosis (day 12) of renal tubules, hypocellularity of hematopoietic tissue, and disaggregation of melanomacrophage aggregates. Fish treated with $5.0 \mathrm{mg} / \mathrm{kg}$ TCB had aneurysm of gill tissue, and both TCB doses elicited hyperplasia of epithelial cells between the secondary lamellae of gills. Treatment with $5.0 \mathrm{mg} / \mathrm{kg}$ TCB caused an apparent tail fin erosion in fish sampled on days 12 and 16, which was detectable in histological sections as inflammation and necrosis in epithelial tissue and an erosion of the calcified bone matrix. Plasma chemistry analysis indicated that treatment with $0.1 \mathrm{mg} / \mathrm{kg} \mathrm{TCB}$ caused no change in the concentrations of electrolytes or nitrogenous waste products, but did cause an elevation in aspartate aminotransferase. Most histological alterations were induced by both $0.1 \mathrm{mg} / \mathrm{kg}$ and $5.0 \mathrm{mg} / \mathrm{kg} \mathrm{TCB}$, but pathology was generally more severe or more extensive in tissues of fish treated with the higher dose. At both TCB doses, organ damage increased with time from day 1 to day 12 . Among the fish treated with $5.0 \mathrm{mg} / \mathrm{kg}$, organ damage was equally severe (on average) on days 12 and 16, despite some TCB depuration by day 16 . Among the fish treated with $0.1 \mathrm{mg} / \mathrm{kg}$, organ damage was less severe in fish sampled on day 16 than on day 12 . This lessening of pathology was correlated with substantial TCB depuration from tissues between days 5 and 16, and may suggest repair of some lesions. Mortality was seen among the fish treated with $5.0 \mathrm{mg} / \mathrm{kg} \mathrm{TCB}$, possibly due an impairment of kidney and gill functions. 


\section{Introduction}

Polychlorinated biphenyls (PCB) are ubiquitous and persistent contaminants in the marine environment (Tanabe, 1988; Fowler, 1990), that elicit a wide range of toxic effects in mammals including reproductive impairment, neurotoxic, genotoxic, hepatotoxic, and immunotoxic effects (reviewed in Safe, 1984; Safe, 1992). Far less is known about PCB toxicity in teleost species, but effects are thought to include growth inhibition, impaired bone development, reproductive impairment (reviewed in McFarland and Clark, 1989), and immune suppression (Spitsbergen et al., 1988c).

Liver histopathology in PCB-exposed fish has been examined in several studies. Some investigators have observed degenerative changes in liver of fish from PCBcontaminated sites, including preneoplastic and neoplastic lesions (Myers et al., 1992; Dey et al., 1993), increase in size and number of melanomacrophage centers, hepatocellular hypertrophy, increased fatty vacuolation, and cell necrosis (Pierce et al., 1978). Fewer investigations have described liver pathology induced by controlled laboratory exposure of fish to PCB mixtures (Aroclors). Such studies have documented vacuolation of hepatocytes, dilation of rough endoplasmic reticulum in hepatocytes (Sivarajah et al., 1978), irregular structure of hepatocyte nuclei (Hacking et al., 1978), and increased content of smooth endoplasmic reticulum in hepatocytes (Klaunig et al., 1979). In contrast to the thorough investigations of teleost liver, very little information is available concerning PCB histopathology in extrahepatic organs of fish, and to our knowledge no studies have examined cellular alterations associated with exposure of fish to individual PCB congeners.

3,3',4,4'-Tetrachlorobiphenyl (TCB) is a coplanar PCB congener and an Ah receptor agonist, thus belonging to the class of PCBs that elicit TCDD-like toxicity in mammals (reviewed in Goldstein and Safe, 1989). TCB is a potent inducer of the CYP1A1 homologue in fish (Gooch et al., 1989), that is metabolized slowly by teleost CYP1A1 to aqueous-soluble products in vitro and in vivo (chapter 5). In the present study we treated the marine fish scup (Stenotomus chrysops) with TCB at two doses, $0.1 \mathrm{mg} / \mathrm{kg}$ and $5.0 \mathrm{mg} / \mathrm{kg}$, and killed animals over a 16 day time-course for examination of histopathology under light microscopy. TCB treatment induced lesions and/or cellular alterations in liver, spleen, kidney, gill, and tail fin. Most alterations were seen to occur at both TCB doses, although pathology was generally more severe or more extensive in fish exposed to the higher dose. The severity of lesions increased with time from day 1 to day 12 at both doses. Tissues of fish treated with the low dose and sampled on day 16 were little different from controls, possibly suggesting that repair of lesions occurred concomitantly with TCB depuration. 


\section{Materials and Methods}

Chemicals 3,3',4,4'-Tetrachlorobiphenyl (TCB, IUPAC \#77) was purchased from Pathfinder (St. Louis, MO). Purity of TCB was greater than $99 \%$ by GC/ECD and GC/MS analysis. Further analysis by high resolution (5000 resolution) mass spectrometry (VG Autospec) and selective ion monitoring showed that the potent Ah receptor agonist $3,3^{\prime}, 4,4^{\prime}, 5$-pentachlorobiphenyl was not a contaminant of the TCB, with a detection limit of $0.001 \%$.

Animals and Dosing Scup (Stenotomus chrysops)were caught by angling in Vineyard Sound, MA in August, 1991. Fish were held in flowing seawater tanks at $20^{\circ} \mathrm{C}$ for fourteen months prior to experimental use, and were maintained on a diet of Purina trout chow and frozen squid. Prior studies in our laboratory indicate that the bulk of environmental contaminants are depurated from the tissues of fish that are held in aquaria for one year, and that these fish show little CYP1A1 expression. At the time of the dosing, experimental animals (mixed sex) were gonadally immature and weighed 76 - 276 grams. Individuals were injected intraperitoneally with either corn oil (vehicle controls) or with 0.1

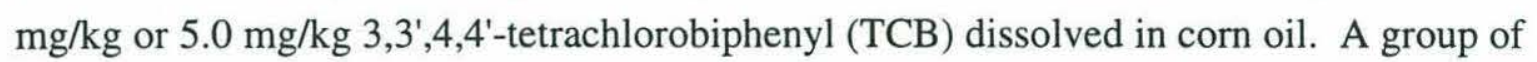
uninjected fish was used as a "zero" point in the time-course. Individuals were held in flowing seawater tanks at $20^{\circ} \mathrm{C}$ until sampling ( 1 day to 18 days) or until death from toxicity. Fish were fed Purina trout chow once weekly, and were never fed within the three days prior to killing. At each time point, a group of 4 or 5 animals from each treatment group were killed by cervical transection and dissected immediately.

Histology Sections of liver, posterior kidney, gonad, heart, gill, spleen, intestinal caecum, and intestinal duodenum were removed from animals upon killing and immediately fixed in $10 \%$ neutral buffered formalin ( $\mathrm{pH}$ 7.4). Spleen was sampled on days 0,12 , and $16 / 18$ only, all other tissues were sampled at each time point. In addition, sections of tail fin were dissected from some fish sampled on day $16 / 18$, and these were de-calcified prior to embedding. After one week of fixation, tissues were embedded in paraffin using standard methods (Luna, 1968). Five $\mu \mathrm{m}$ sections were cut from paraffin blocks, mounted on slides, and stained with hematoxylin and eosin (H\&E). H\&E-stained slides were examined under light microscopy for evidence of histopathology in all tissues. 
Scoring of histopathology In the organs in which TCB treatment consistently elicited histopathology (liver, spleen, kidney, and gill), subjective indices were developed to measure the degree of alteration. Liver sections were scored for basophilia of hepatocytes, as follows:

(0) hepatocyte cytoplasm is mostly white, apparently full of lipid or glycogen;

(1) hepatocyte cytoplasm has diffuse, light purple coloring with some white patches;

(2) hepatocyte cytoplasm is filled with hazy, purple/pink coloring under H\&E stain;

(3) hepatocyte cytoplasm is deep purple/blue under H\&E stain.

The number of melanomacrophage centers in spleen sections was determined by counting the number of discrete aggregates, on 100x magnification, for three randomly selected fields on each slide. A number was determined for each individual fish spleen by averaging the three counts.

Kidney sections were scored for severity of renal tubule lesions, as follows:

(0) tubules are composed of normally-shaped cuboidal cells, most have small lumen;

(1) tubule epithelia are still cuboidal, but many or most tubules have dilated lumen;

(2) many tubule epithelia are flattened and some are necrotic, most tubules are dilated;

(3) tubules contain both flattened (degenerative) and cuboidal (regenerative) epithelia, most tubules have necrotic epithelia and debris in lumen;

(4) similar to (3), but almost all tubules have necrotic epithelia.

Kidney sections were also scored for depletion of hematopoietic tissue, as follows:

(0) compact arrangement of hematopoietic cells, occurring between most renal tubules;

(1) loose arrangement of hematopoietic cells, resulting in clusters of renal tubules without hematopoietic cells among them;

(2) multifocal arrangement of hematopoietic cells, with many small patches in 100x field;

(3) sparse arrangement of hematopoietic cells, with few small patches in 100x field.

Gill sections were scored for hyperplasia of epithelial cells, as follows:

(0) a thin layer or double layer of epithelial cells surrounds each secondary lamella;

(1) small foci of epithelial hyperplasia occur between few secondary lamellae, on few primary gill filaments, causing fusion of adjacent secondary lamellae in these foci;

(2) foci of hyperplasia occur on moderate number of secondary lamellae, mostly at the ends of the primary filament;

(3) foci of hyperplasia occur between many secondary lamellae, from base to end of the primary filament;

(4) epithelial hyperplasia is diffuse, resulting in adhesion of almost all secondary lamellae. 
Plasma chemistry In a separate experiment, 3 scup were treated by intraperitoneal injection with corn oil and 4 were treated with $0.1 \mathrm{mg} / \mathrm{kg}$ TCB in corn oil. Blood samples were drawn from all fish on day 12 through the caudal peduncle vein, using sterile syringes pretreated with lithium heparin $(10 \mathrm{mg} / \mathrm{ml}$ solution). Samples were centrifuged on low speed to separate cells from plasma, and plasma was stored at $-20^{\circ} \mathrm{C}$ until analysis. Plasma chemistry was performed at the New York State Diagnostic Laboratory, Cornell University College of Veterinary Medicine, using a standard screening panel at $37^{\circ} \mathrm{C}$.

Photographs $35 \mathrm{~mm}$ color slides (EPY 64 Tungsten Ektachrome) were taken of H\&Estained microscope slides and scanned into Adobe Photoshop software (v. 2.5.1 for Macintosh) using a Kodak RFS 2035 slide scanner. Images were scaled and labeled using Adobe Photoshop, and were printed on a Kodak XL photographic printer. No image enhancement, contrast adjustment, or color adjustment was used to modify the figures.

TCB residues Content of 3,3',4,4'-tetrachlorobiphenyl was quantified in pooled samples, consisting of liver or muscle sections from all fish within a treatment group. Subcutaneous muscle tissue was sampled from the side of body, mid-way between the head and tail, just below the lateral line. Tissue extraction followed AOAC Method 983.21 (Helrich, 1990). Samples were mixed with sodium sulfate and pet ether, homogenized, and serially extracted with pet ether. Extracts were cleaned up on a Florisil column and concentrated under nitrogen. Extracts were analyzed for TCB by gas chromatography with electron capture detection (GC/ECD) using an HP 5890 Series II GC/ECD. Confirmation of the TCB was performed by gas chromatography with mass spectrometry (GC/MS) using an HP 5890/5970 GC/MS by monitoring molecular ions 110, 150, 184, 220, 222, 290, 292 , and 294. The fragmentation pattern of each sample was compared to the TCB standard and TCB was quantified with $\mathrm{m} / \mathrm{z} 292$. Measured concentrations of TCB were corrected for extraction efficiency using the recovery of 4,4'-dibromooctafluorobiphenyl (DBOFB) which was spiked into each sample prior to extraction, and were quantified relative to the internal standard 2,4,5,6-tetrachloro-m-xylene (TCMX). Recoveries of DBOFB ranged from 60 to $96 \%$ with an average of $76 \%$. The average recovery of TCB matrix spikes was $110 \%$. The precision of duplicate analyses was within 3\%. Data were obtained as TCB content per g tissue wet weight. Sample dry weight and sample lipid weight were measured for a few representative tissue samples. 


\section{Results}

\section{Cumulative mortality}

None of the fish treated with either vehicle or with $0.1 \mathrm{mg} / \mathrm{kg}$ TCB died over the course of the experiment. Among the fish treated with $5.0 \mathrm{mg} / \mathrm{kg} \mathrm{TCB}$, the rate of mortality increased with time following injection (Fig 1). Thus, between days 0 and 4 none of the treated fish died; between days 4 and 5 only one fish died out of a total of 20 that were treated with this dose and not yet sampled; between days 5 and 12, three fish died out of the 14 remaining; and between days 12 and 16 one-third of the remaining fish (2/6) died. The increase in mortality with time may be attributed to a severe deterioration of kidney and gill tissue, and possible loss of function, following treatment with $5.0 \mathrm{mg} / \mathrm{kg} \mathrm{TCB}$ (see below).

\section{Gross effects of TCB}

For each animal, a morphometric condition index (body weight/ length squared), hepatosomatic index (liver weight/ body weight) and gonadosomatic index (gonad weight/ body weight) were determined at the time of death. Neither condition index (Fig 2A) nor hepatosomatic index (Fig 2B) was significantly altered by either TCB dose, at any time point following TCB treatment. Gonadosomatic index was also unaffected by the treatments, and the values indicated that animals were not reproductively active at the time of the experiment (males: $0.05 \pm 0.02$; females: $0.52 \pm 0.11$ ).

Gross pathology was noted among the fish treated with $5.0 \mathrm{mg} / \mathrm{kg}$ TCB. On days 12 and 16, most animals treated with this dose had pale gill tissue displaying aneurysm, pale-colored livers, and eroded tail fins. Three fish (1 on day 12, 2 on day 16) had thin, red fluid in the intraperitoneal cavity, and one fish (day 16) had a grossly swollen bladder full of retained urine. None of the experimental animals, including those that died spontaneously, exhibited signs of peritonitis, suggesting that gross pathology and mortality were due to TCB itself and not to improper injection.

\section{Liver histopathology}

A marked basophilia of hepatocytes, indicative of proliferation of endoplasmic reticulum and of a loss of lipid and glycogen reserves, developed in all TCB-treated fish over the time-course of the experiment. This was scored with a subjective index of severity, as described in Methods, and scores for each treatment group are plotted for comparison (Fig 3A). In untreated and control (vehicle-treated) fish, hepatocytes were strongly vacuolated, probably resulting from heavy accumulation of lipid and glycogen, which are artifactually 


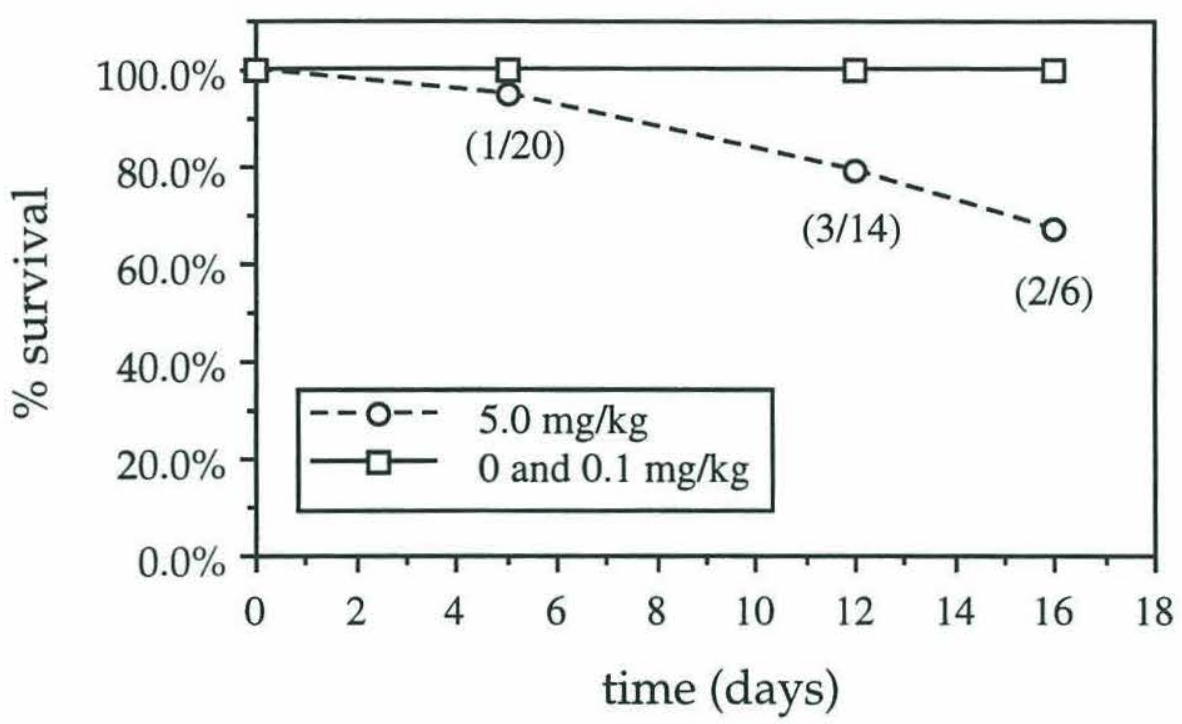

Figure 1. Cumulative mortality in treated fish. $\mathrm{X}$-axis is the number of days subsequent to TCB injection. Plotted on the $y$-axis is the percent survival of treated fish during each sampling "interval" (i.e. the spaces of time between sampling points). Indicated in parenthesis is the number of fish that died during a sampling interval, divided by the number of treated fish alive at the beginning of the interval. 



Figure 2. Condition index (A) and hepatosomatic index (B) in treated fish. Condition index was measured as body weight divided by the square of body length, hepatosomatic index was measured as liver weight divided by body weight. $\mathrm{X}$-axis is number of days subsequent to TCB injection, $y$-axis is index. Each point represents the mean and standard deviation (n-1) of measurements on four or five fish per treatment group. Plot symbols are: square, corn oil control, open circle, $0.1 \mathrm{mg} / \mathrm{kg} \mathrm{TCB}$, closed circle, $5.0 \mathrm{mg} / \mathrm{kg}$ TCB. 
Figure 3. Histopathology in treated fish, based on subjective scores of severity as described in Methods. (A) degree of basophilia in hepatocytes, (B) severity of damage to renal tubules, (C) loss of hematopoietic tissue in kidney, (D) extent of epithelial hyperplasia in gill. $\mathrm{X}$-axis is number of days subsequent to TCB injection, $\mathrm{y}$-axis is score. Each point represents the mean and standard deviation (n-1) of the scores for four or five fish per treatment group. Plot symbols are: square, corn oil control, open circle, $0.1 \mathrm{mg} / \mathrm{kg} \mathrm{TCB}$, closed circle, $5.0 \mathrm{mg} / \mathrm{kg}$ TCB. 
A: basophilia of hepatocytes

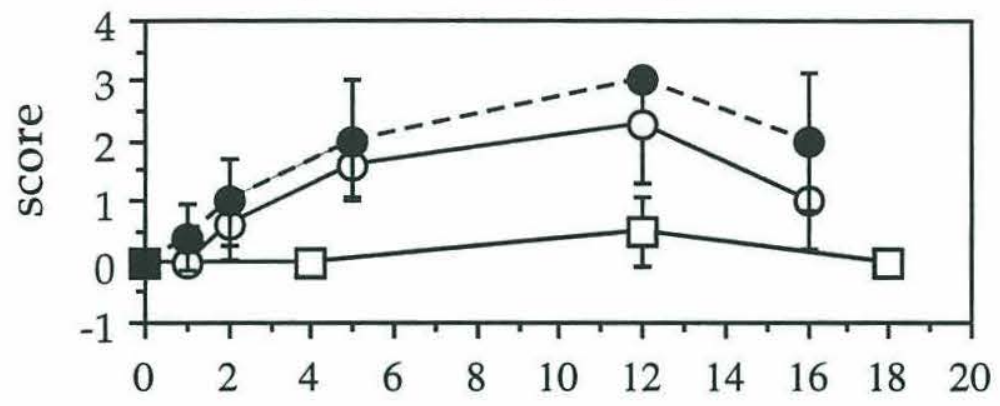

B: renal tubule lesion

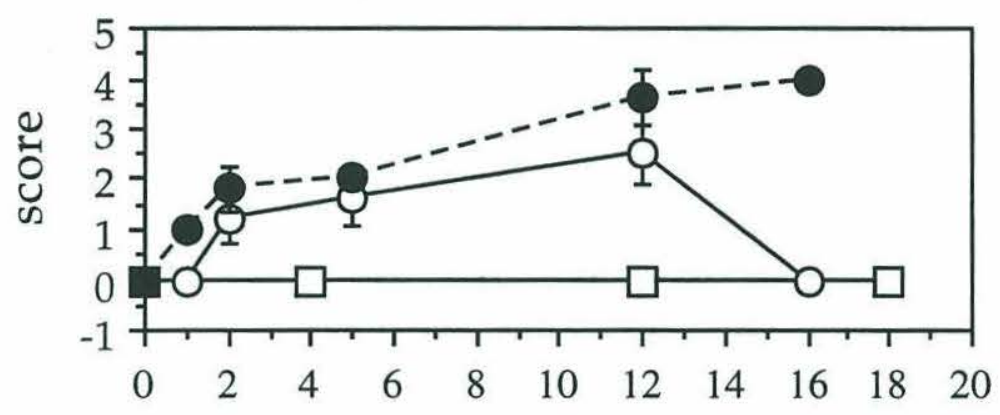

C: hematopoietic tissue hypocellularity



D: hyperplasia of gill epithelia

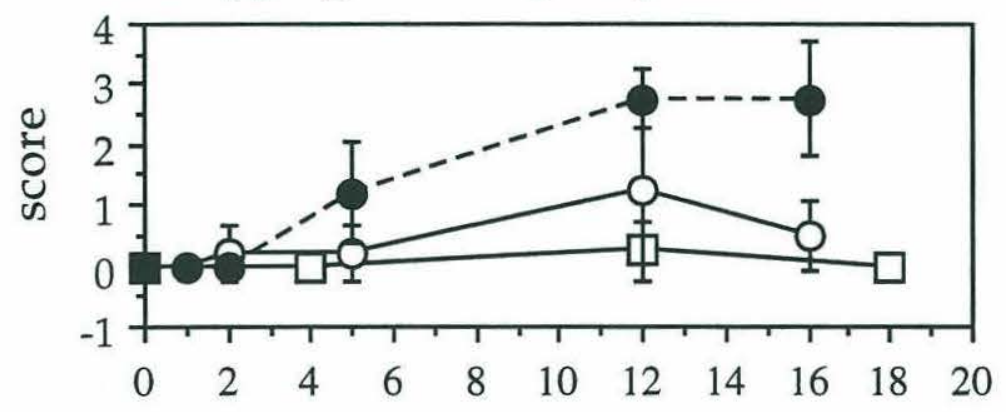

time (days) 
Figure 4. H\&E stained slides of liver and spleen. (A) Liver of fish treated with corn oil and sampled on day 12 (1000x). Note small nuclei and light cytoplasm. (B) Liver of fish treated with $0.1 \mathrm{mg} / \mathrm{kg}$ TCB and sampled on day $12(1000 \mathrm{x})$. Note larger nuclei, diffuse stain in cytoplasm, and cytoplasmic vacuoles (v). (C) Spleen of fish treated with corn oil and sampled on day 12 (200x). Melanomacrophage aggregate is indicated (m). (D) Spleen of fish treated with $5.0 \mathrm{mg} / \mathrm{kg}$ TCB and sampled on day 12 (200x).

Melanomacrophage aggregates are indicated (m). 


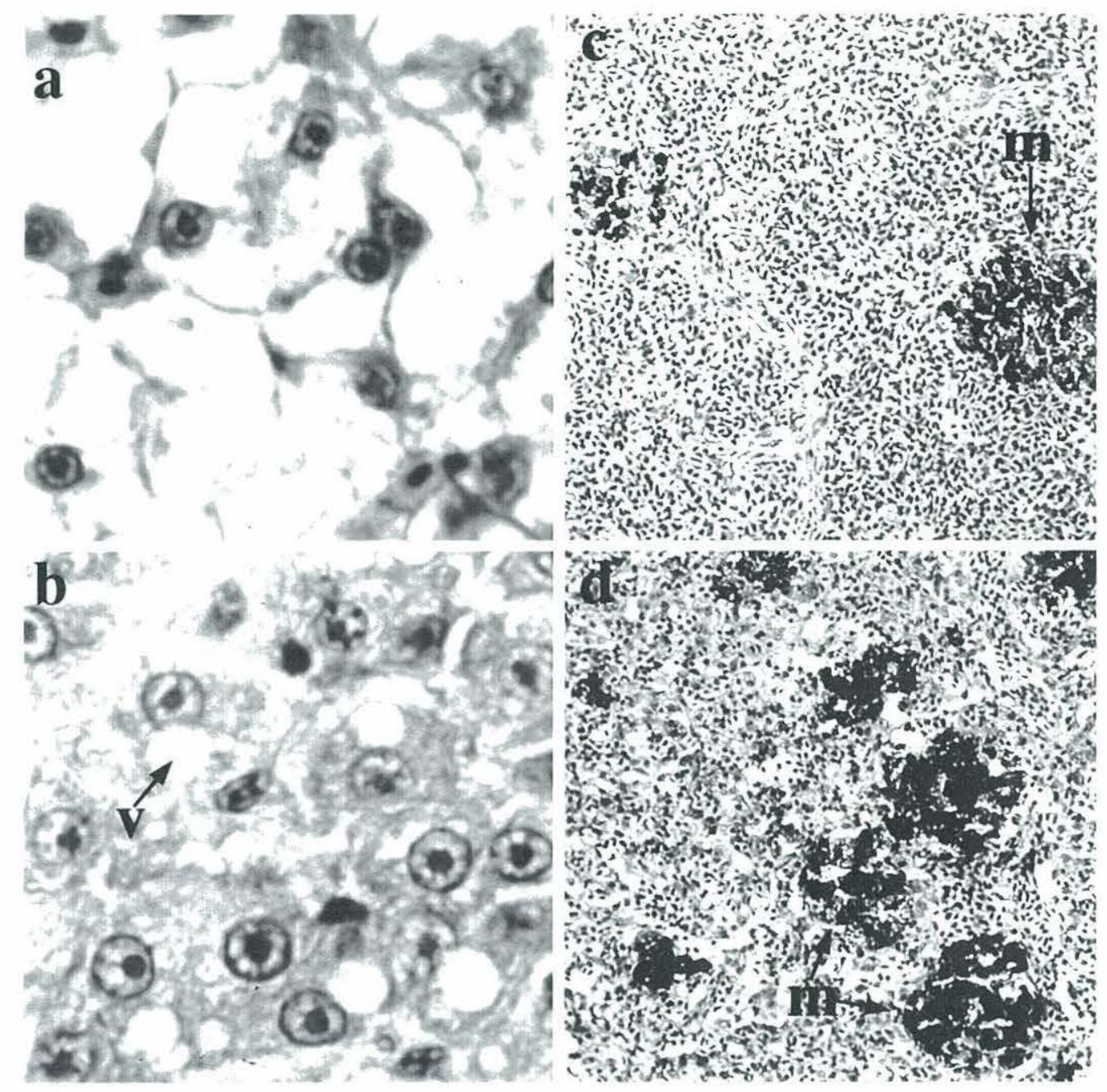


removed during tissue processing. This results in a predominantly light-colored cytoplasm under H\&E stain (Fig 4A). From day one until day five after TCB treatment, a diffuse coloring developed in the cytoplasm of hepatocytes from fish treated with either dose, and by day 12 , an extreme basophilia typified hepatocytes from fish treated with the high dose (Fig 4B). Round cytoplasmic vacuoles, 3-4 $\mu \mathrm{m}$ in diameter, were seen in many TCB treated fish (Fig 4B), which may represent low levels of lipid and glycogen remaining in liver. Irregularly shaped vacuoles were also seen in hepatocytes of TCB treated fish, giving a foamy appearance to the cytoplasm (Fig 4B). The latter vacuoles may be the result of swelling of endoplasmic reticulum or other organelles.

Some TCB treated fish had enlarged, regular nuclei (Fig 4B, as compared to 4A), indicating activity. No changes were detected in hepatic macrophages.

\section{Spleen histopathology}

All fish treated with TCB had an increased number of melanomacrophage aggregates in the spleen (Table 1). Whereas control fish had sparsely distributed melanomacrophage aggregates which were generally round in shape (Fig 4C), TCB treated fish had a larger number of smaller, more irregularly shaped aggregates (Fig 4D). TCB treatment thus brought about an increased number of individual aggregates, but may not have brought about an increased number of melanomacrophage cells per unit area. In fish treated with the high dose, we also observed a more "open" architecture to the spleen (Fig 4D), with extensive acellular space, suggestive of edema.

TABLE 1

Number of Melanomacrophage Aggregates in Spleen of TCB-Treated and Control Fish

\begin{tabular}{cccc}
\hline day & $\begin{array}{c}\text { corn oil / control } \\
(\mathrm{n}=4)\end{array}$ & $\begin{array}{c}0.1 \mathrm{mg} / \mathrm{kg} \mathrm{TCB} \\
(\mathrm{n}=5)\end{array}$ & $\begin{array}{c}5.0 \mathrm{mg} / \mathrm{kg} \text { TCB } \\
(\mathrm{n}=5)\end{array}$ \\
\hline 0 & $38 \pm 12$ & & \\
12 & $39 \pm 8$ & $77 \pm 8$ & $109 \pm 26$ \\
$16 / 18$ & $29 \pm 14$ & $50 \pm 16$ & $95 \pm 43$ \\
\hline
\end{tabular}

For each fish, the number of aggregates was counted in three random fields at $100 \mathrm{x}$, and averaged. Values presented are mean \pm standard deviation for four or five fish in each treatment group. "Day 0" fish were untreated, TCB-treated fish were killed on days 12 and 16 , and corn oil treated-fish were killed on days 12 and 18 . 
Kidney histopathology

TCB treatment induced a marked necrosis of renal tubules (Fig 3B), which was scored in each fish with a subjective index of severity, as described in Methods. In untreated and vehicle-treated fish, renal tubules were characterized by an even layer of cuboidal cells and a small lumen, clusters of hematopoietic cells were found throughout the tissue and surrounding most tubules, and macrophages occurred in discrete, round-shaped aggregates (Fig 5A). Following TCB treatment there was a dilation of the renal tubule lumen (day 12), followed by a squamous metaplasia, or flattening, of tubule epithelia (Fig 5B; day 5), which implies necrosis and shedding of renal epithelial cells. Also seen in this figure are cross-lumenal tags connecting epithelial cells on opposite sides of the tubule, which characterized many TCB-treated fish kidneys. By day 12 (Fig 5C), kidneys displayed chronic necrosis mixed with regeneration. Degeneration was indicated by foci of squamous epithelia, and regeneration was indicated by the presence of large, basophilic, cuboidal epithelia in many tubules. Also seen in this figure is mineralized, intralumenal debris, which was typical for kidneys of fish treated with $5.0 \mathrm{mg} / \mathrm{kg}$ and sampled on day 12. Such condensed debris may be associated with tubule dilation and obstruction. Vacuolation within tubule epithelia was seen on day 12, suggesting fluid accumulation in the cells.

A loss of hematopoietic tissue accompanied kidney tubule degeneration. This was also scored over time with a subjective index, as described in Methods (Fig 3C). The quantity of renal hematopoietic tissue ranged from a continuous network (Fig 5A; control), to a multifocal distribution (Fig 5B; day 5), to a sparse distribution (Fig 5C; day 12). A similar depletion of kidney hematopoietic tissue was induced by TCDD in rainbow trout, perch, and lake trout sac fry (Spitsbergen et al., 1988a; Spitsbergen et al., 1988b; Spitsbergen et al., 1991). By day 12, kidneys were characterized by extensive acellular space between tubules and within foci of hematopoietic tissue, suggesting edema (Fig 5C). Melanomacrophage centers in kidney tended to disaggregate in TCB-treated fish, changing from discrete, round structures (control) into less-round, more granular structures (Fig 5B; day 5), and in some cases dispersing (day 12). Renal pathology occurred at both TCB doses, although it was generally more severe at the high dose (Fig $3 \mathrm{~B}$ and $3 \mathrm{C}$ ). By day 16 , kidneys of all fish treated with the low dose showed little pathology and resembled the kidneys of control fish.

\section{Gill histopathology}

Treatment with either dose of TCB was associated with a hyperplasia of epithelial cells between the secondary lamellae; this was more extensive at the high dose. The extent of 
Figure 5. H\&E stained slides of kidney and gill. (A) Kidney of fish treated with corn oil and sampled on day $12(400 x)$. Section showing renal tubules ( $\mathrm{T}$ in lumen), hematopoietic cells surrounding tubules. (B) Kidney of fish treated with $5.0 \mathrm{mg} / \mathrm{kg}$ TCB and sampled on day $5(400 x)$. Section showing dilated renal tubules with squamous epithelia ( $\mathrm{T}$ in lumen), melanomacrophage aggregate in lower left, foci of hematopoietic cells. (C) Kidney of fish treated with $5.0 \mathrm{mg} / \mathrm{kg}$ TCB and sampled on day $12(400 \mathrm{x})$. Section showing necrotic renal tubules ( $\mathrm{T}$ in lumen) with intralumenal debris, small patch of hematopoietic cells in lower right. (D) Gill of fish treated with corn oil and sampled on day $12(400 x)$. Section showing secondary gill lamellae on one primary filament. (E) Gill of fish treated with $0.1 \mathrm{mg} / \mathrm{kg}$ TCB and sampled on day $12(400 \mathrm{x})$. Note hyperplasia of epithelial cells along primary lamella, causing adhesion at base of secondary lamellae $(*$ indicates the outer edge of hyperplasia). (F) Gill of fish treated with $5.0 \mathrm{mg} / \mathrm{kg}$ TCB and sampled on day 12 (400x). Arrows indicate the base and tip of each secondary lamella. Note hyperplasia of epithelial cells causing severe, diffuse adhesion of secondary lamellae (* indicates the outer edge of hyperplasia). 


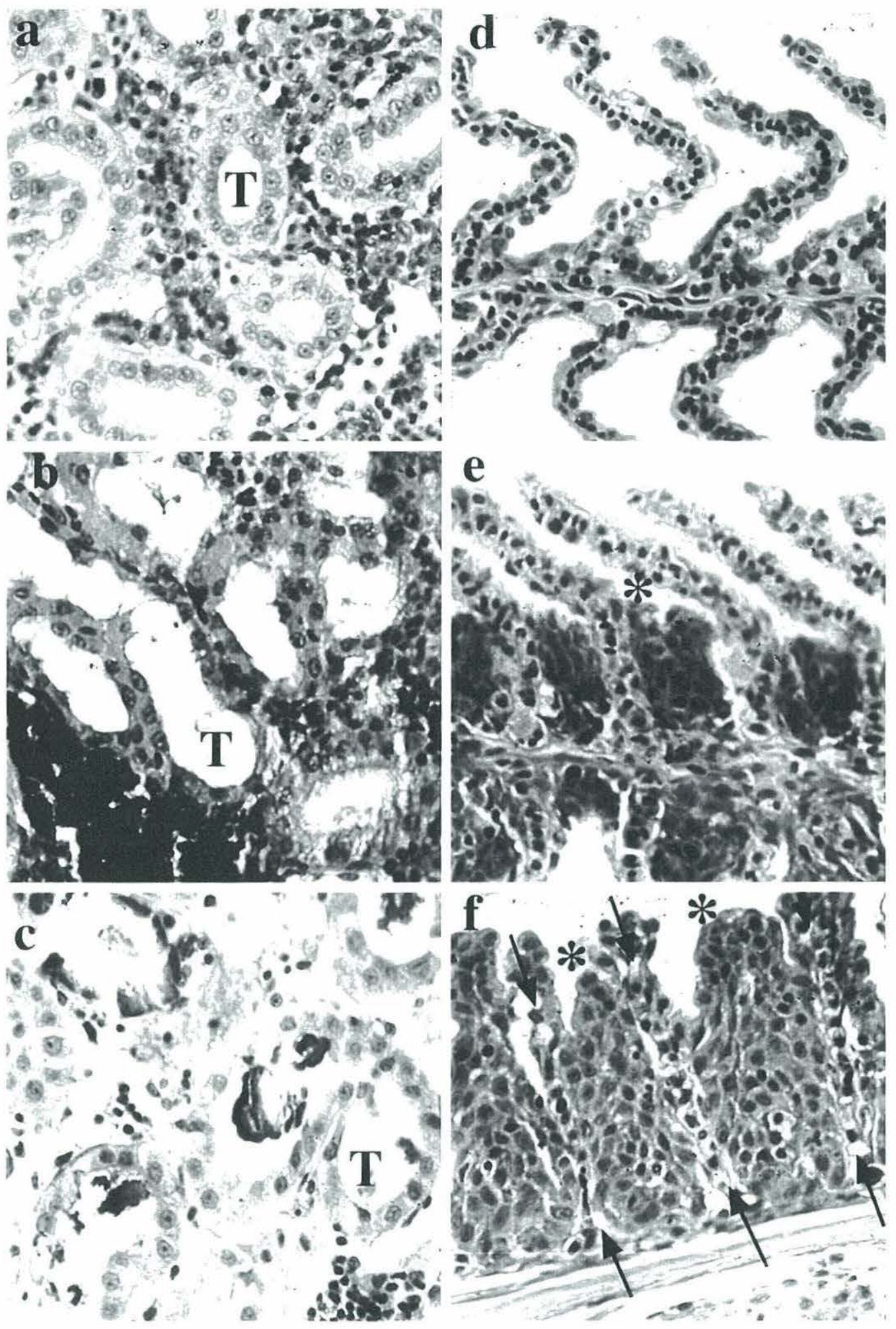


Figure 6. Scup tail fin. (A) Vehicle-treated scup showing no fin erosion (top), and TCBtreated scup showing erosion of tail fin (bottom). (B) Superficial epithelial tissue from tail fin of fish displaying gross signs of fin erosion, treated with $5.0 \mathrm{mg} / \mathrm{kg} \mathrm{TCB}$ and sampled on day 16 (400x). Section showing necrotic and swollen epithelia (arrows on some examples). (C) Calcified bone tissue from tip of tail fin, from same fish shown in B (100x). Note the absence of cartilage and epithelia surrounding the ray, erosion along the border and within the matrix of the ray (arrows). (D) Tail fin of fish treated with corn oil and sampled on day 18 (100x). Shown is segmented bone tissue (at center) surrounded by layers of cartilage. 

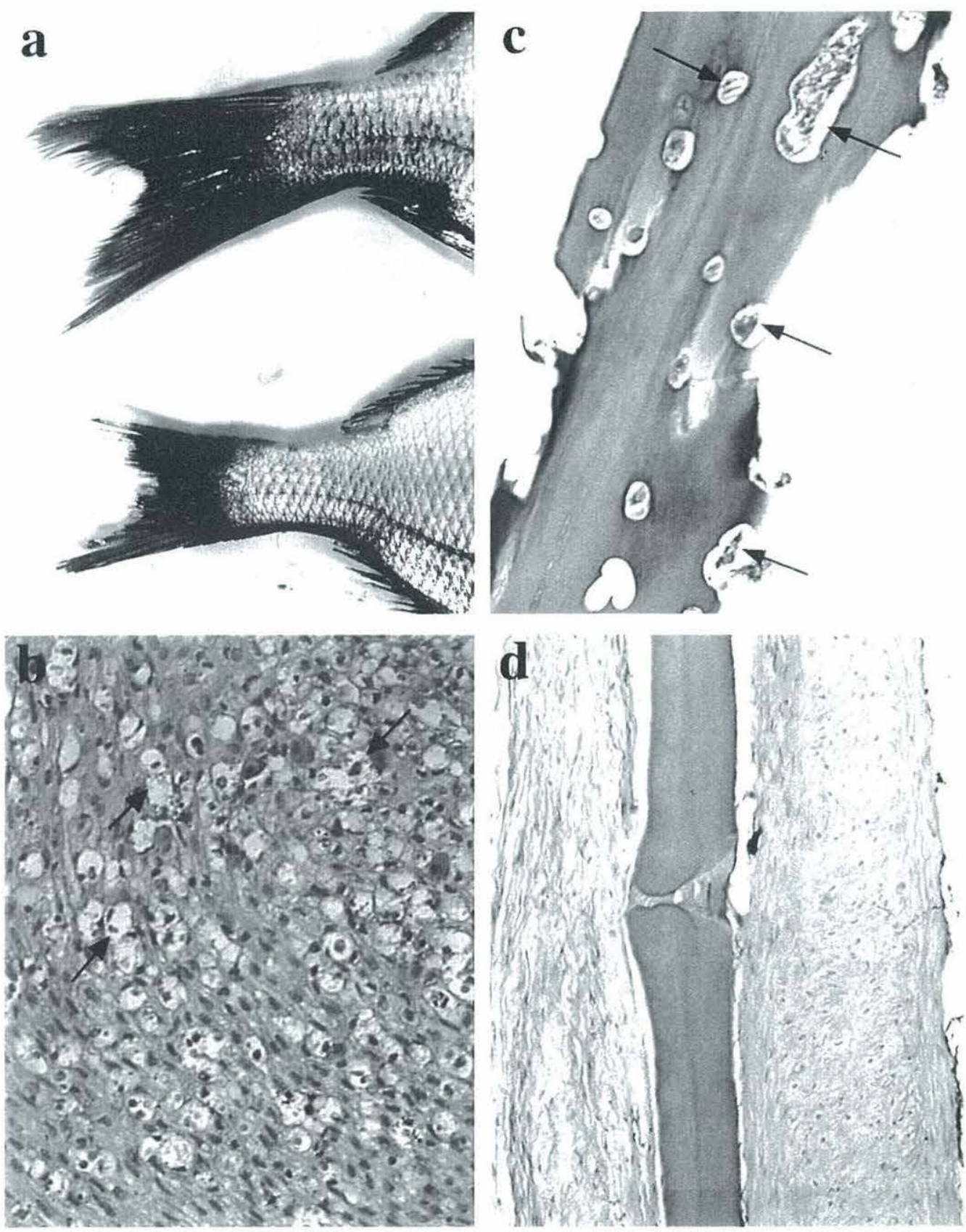
revealed several alterations. Many superficial epithelia were necrotic, swollen, or vacuolated (Fig 6B). Both epithelial and cartilaginous tissue were eroded away at the tips of the fin, leaving exposed bone tissue (Fig 6C). In areas of bone that were thus exposed, irregular erosions occurred along the border and within the calcified bone matrix (Fig 6C). Many of these eroded spaces contained cell debris and possible inflammatory cells, which were also found in the epithelia. In tail fin of the vehicle-treated fish sampled on day 18 , no epithelial necrosis was noted, nor did we see a loss of soft tissues around bone, or an erosion of bony tissues (Fig 6D).

\section{Histology of other tissues}

In most organs examined, vascular endothelium appeared to be histologically normal. TCB induced no apparent histological damage in most tissues of mesenchymal origin (muscle, heart), nor in gonad or intestinal mucosa.

\section{Plasma chemistry}

In a separate experiment, fish were treated with either vehicle or $0.1 \mathrm{mg} / \mathrm{kg} \mathrm{TCB}$, and plasma samples were drawn twelve days after treatment for chemical analysis (Table 2). The only parameter that was apparently affected by TCB treatment was aspartate aminotransferase (AST), which was elevated above control levels in two of the four TCBtreated fish. Elevation in AST has been associated with hepatic disease in fish (Casillas et al., 1982). All other components analyzed in TCB-treated fish fell within the range of concentrations seen in vehicle-treated fish.

\section{$\underline{\text { Residue analysis }}$}

TCB content was measured in pooled samples of liver and muscle tissue from each group of scup in the time-course study (Fig 7; Table 3). The concentration of TCB in muscle tissue reached a high peak in fish sampled one day after injection with either dose, and this dropped sharply by day $5(0.1 \mathrm{mg} / \mathrm{kg}$ dose $)$ or by day $2(5.0 \mathrm{mg} / \mathrm{kg}$ dose $)$. In fish treated with $0.1 \mathrm{mg} / \mathrm{kg}$, the TCB content in liver reached a maximum concentration of $0.015 \mu \mathrm{g} / \mathrm{g}$ liver dry weight on day 5 . In fish treated with $5.0 \mathrm{mg} / \mathrm{kg}$, the TCB content in liver did not reach a maximum until day 12 , at which time the concentration was $2.2 \mu \mathrm{g} \mathrm{TCB} / \mathrm{g}$ liver dry weight. At both doses, hepatic TCB content on day 16 was substantially lower than the hepatic peak concentration, indicating that the bulk of the dose was depurated over the time-course of the experiment. Analysis of bile residues indicated that this depuration was due to both metabolism and to the loss of parent compound (White et al., in preparation). 


\section{Plasma chemistry}

In a separate experiment, fish were treated with either vehicle or $0.1 \mathrm{mg} / \mathrm{kg} \mathrm{TCB}$, and plasma samples were drawn twelve days after treatment for chemical analysis (Table 2). The only parameter that was apparently affected by TCB treatment was aspartate aminotransferase (AST), which was elevated above control levels in two of the four TCBtreated fish. Elevation in AST has been associated with hepatic disease in fish (Casillas et al., 1982). All other components analyzed in TCB-treated fish fell within the range of concentrations seen in vehicle-treated fish.

\section{$\underline{\text { Residue analysis }}$}

TCB content was measured in pooled samples of liver and muscle tissue from each group of scup in the time-course study (Fig 7; Table 3). The concentration of TCB in muscle tissue reached a high peak in fish sampled one day after injection with either dose, and this dropped sharply by day $5(0.1 \mathrm{mg} / \mathrm{kg}$ dose $)$ or by day $2(5.0 \mathrm{mg} / \mathrm{kg}$ dose $)$. In fish treated with $0.1 \mathrm{mg} / \mathrm{kg}$, the TCB content in liver reached a maximum concentration of $0.015 \mu \mathrm{g} / \mathrm{g}$ liver dry weight on day 5 . In fish treated with $5.0 \mathrm{mg} / \mathrm{kg}$, the TCB content in liver did not reach a maximum until day 12 , at which time the concentration was $2.2 \mu \mathrm{g} \mathrm{TCB} / \mathrm{g}$ liver dry weight. At both doses, hepatic TCB content on day 16 was substantially lower than the hepatic peak concentration, indicating that the bulk of the dose was depurated over the time-course of the experiment. Analysis of bile residues indicated that this depuration was due to both metabolism and to the loss of parent compound (White et al., in preparation).

Administered TCB apparently distributed to muscle tissue first, and to liver later, at both doses. This is consistent with prior studies in our laboratory (Harris et al., unpublished data), in which fish were treated orally with 3,3',4,4',5,5'hexachlorobiphenyl (HCB). In that study, $\mathrm{HCB}$ concentration was relatively high in muscle tissue and low in liver at an early sampling point (day 2), followed by a reduced concentration in muscle with redistribution of $\mathrm{HCB}$ to other tissues including liver at a later sampling point (day 6).

TCB concentration in muscle tissue was greater than that in liver tissue in almost every treatment group, when expressed as $\mu \mathrm{g} \mathrm{TCB} / \mathrm{g}$ tissue dry weight (Table 3, top). Average lipid weights in the samples were $3.4 \%$ lipid in liver and $11.7 \%$ lipid in muscle. Thus, the much greater lipid content in muscle could account for the greater TCB concentration in this tissue, as PCBs tend to partition into lipid (Chiou, 1985). When TCB concentrations were expressed as $\mu \mathrm{g} \mathrm{TCB} / \mathrm{g}$ tissue lipid weight (Table 3, bottom), liver concentrations exceeded muscle concentrations at several time points (Fig 7). 
TABLE 2

Plasma Chemistry in Vehicle-Treated and TCB-Treated $(0.1 \mathrm{mg} / \mathrm{kg})$ Fish

\begin{tabular}{|c|c|c|c|c|}
\hline & vehicle $(n=3)$ & & $\mathrm{TCB}(\mathrm{n}=4)$ & \\
\hline & average & st dev & average & st dev \\
\hline sodium $a$ & 151 & 7 & 159 & 8 \\
\hline potassium $a$ & 5.6 & 0.5 & 4.9 & 0.8 \\
\hline chloride $^{a}$ & 149 & 6 & 153 & 7 \\
\hline $\mathrm{CO}_{2}{ }^{a}$ & 6 & 2 & 7 & 1 \\
\hline calcium $b$ & 9.6 & 0.4 & 10.5 & 0.7 \\
\hline phosphorus $b$ & 6.7 & 1.6 & 6.4 & 0.7 \\
\hline total protein $c$ & 1.7 & 0.3 & 1.8 & 0.4 \\
\hline albumin $c$ & 0.3 & 0.1 & 0.3 & 0.0 \\
\hline globulin $c$ & 1.4 & 0.3 & 1.5 & 0.3 \\
\hline urea $b$ & 8.0 & 3.6 & 4.8 & 1.3 \\
\hline creatinine $b$ & 0.2 & 0.1 & 0.2 & 0.1 \\
\hline glucose $b$ & 77 & 15 & 99 & 14 \\
\hline cholesterol $b$ & 112 & 52 & 125 & 50 \\
\hline $\mathrm{ALT}^{d}$ & 3 & 2 & 3 & 1 \\
\hline $\mathrm{AST}^{d}$ & 44 & 17 & 89 & 51 \\
\hline Alk Phos $d$ & 6 & 8 & 12 & 2 \\
\hline GGT $d$ & 1 & 1 & 1 & 2 \\
\hline bilirubin $b$ & 0.6 & 0.3 & 0.5 & 0.4 \\
\hline uric acid $b$ & 0.1 & 0.0 & 0.1 & 0.1 \\
\hline
\end{tabular}

Blood was drawn from the caudal peduncle vein, 12 days after intraperitoneal treatment with either corn oil or TCB in corn oil, and plasma was separated from cells. Plasma components were analyzed with a standard screening panel at $37^{\circ} \mathrm{C}$. Units: $a \mathrm{mEq} / \mathrm{L} ; b \mathrm{mg} / \mathrm{dL} ;{ }^{c} \mathrm{~g} / \mathrm{dL} ; d \mathrm{U} / \mathrm{L}$.

Abbreviations: ALT, alanine aminotransferase; AST, aspartate aminotransferase; Alk Phos, alkaline phosphatase; GGT, gamma glutamyl transferase. 
TABLE 3

TCB Concentration in Pooled Tissue Samples of TCB-Treated Scup

\begin{tabular}{|c|c|c|c|c|}
\hline \multirow[b]{2}{*}{ day } & \multicolumn{2}{|c|}{$\underline{0.1 \mathrm{mg} / \mathrm{kg}}$} & \multicolumn{2}{|c|}{$\underline{5.0 \mathrm{mg} / \mathrm{kg}}$} \\
\hline & liver & muscle & liver & muscle \\
\hline & \multicolumn{2}{|c|}{$\mu \mathrm{g} / \mathrm{g}$ dry weight } & \multicolumn{2}{|c|}{$\mu \mathrm{g} / \mathrm{g}$ dry weight } \\
\hline 0 & 0.0000 & 0.000 & 0.000 & 0.00 \\
\hline 1 & 0.0003 & 0.412 & 0.302 & 8.41 \\
\hline 2 & 0.0023 & 0.171 & 0.411 & 1.09 \\
\hline 5 & 0.0147 & 0.038 & 0.638 & 0.90 \\
\hline 12 & 0.0056 & 0.020 & 2.191 & 1.25 \\
\hline 16 & 0.0059 & 0.012 & 0.404 & 2.29 \\
\hline & \multicolumn{2}{|c|}{$\mu \mathrm{g} / \mathrm{g}$ lipid weight } & \multicolumn{2}{|c|}{$\mu \mathrm{g} / \mathrm{g}$ lipid weight } \\
\hline 0 & 0.000 & 0.000 & 0.00 & 0.00 \\
\hline 1 & 0.002 & 0.926 & 2.22 & 18.90 \\
\hline 2 & 0.017 & 0.384 & 3.02 & 2.45 \\
\hline 5 & 0.108 & 0.085 & 4.69 & 2.02 \\
\hline 12 & 0.041 & 0.045 & 16.11 & 2.81 \\
\hline 16 & 0.043 & 0.027 & 2.97 & 5.14 \\
\hline
\end{tabular}

Fish were treated with TCB by a single intraperitoneal injection on day 0 , and sampled at the time points indicated. TCB concentration was measured by GC/ECD as described in Methods, in pooled samples consisting of equally-sized tissue sections from all fish within a treatment group ( $n=4$ or 5 fish per sample). TCB concentration is expressed in $\mu \mathrm{g}$ TCB/ g tissue dry weight (top) or $\mu \mathrm{g} \mathrm{TCB} / \mathrm{g}$ tissue lipid weight (bottom). Concentrations per lipid weight were calculated by converting dry weight to wet weight (dry $=25 \%$ of wet for liver, dry $=26.3 \%$ of wet for muscle), then dividing by percent lipid in wet tissue $(3.4 \%$ lipid in liver, $11.7 \%$ lipid in muscle). 

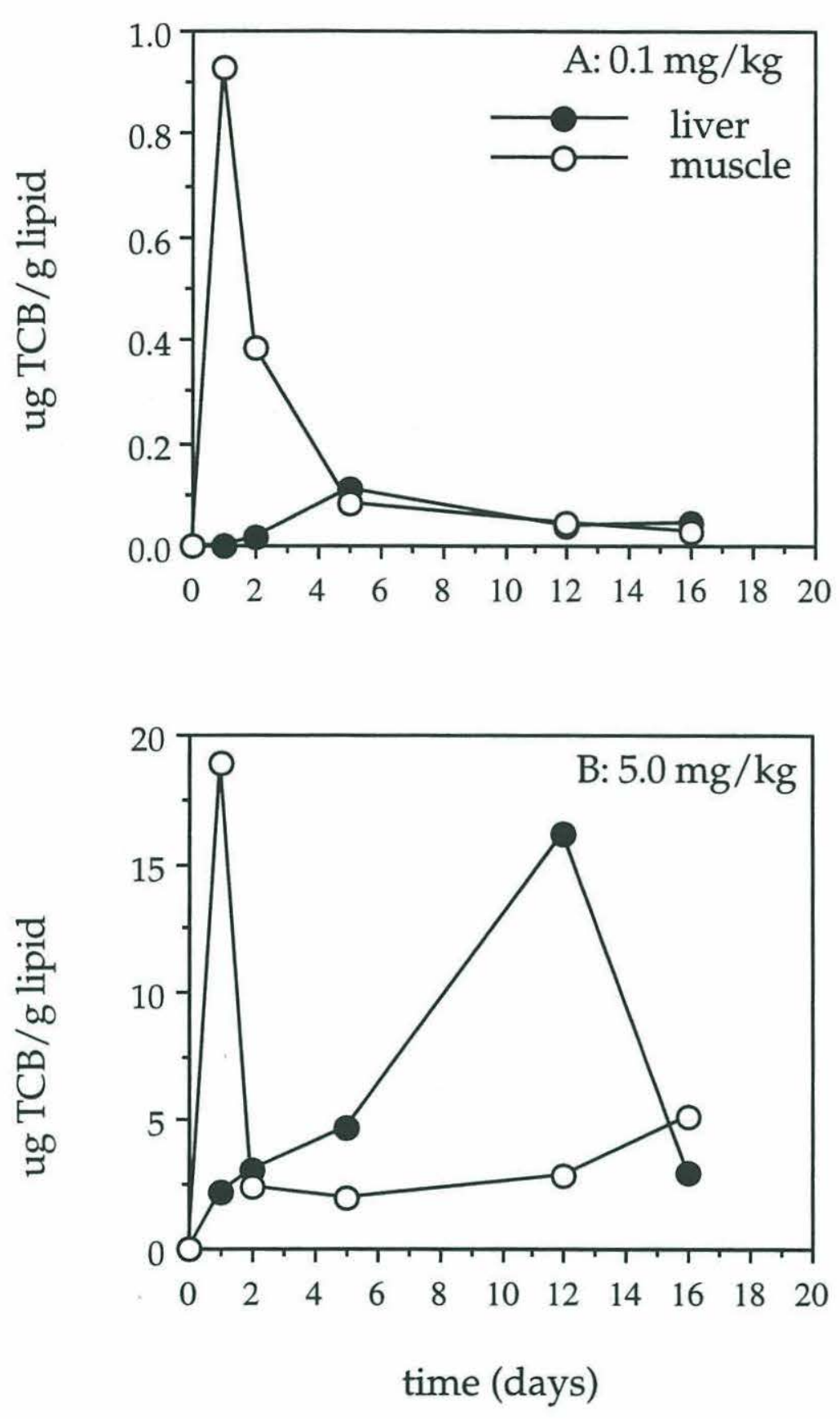

Figure 7. GC/MS analyses of TCB content in pooled liver and muscle samples from fish treated with $0.1 \mathrm{mg} / \mathrm{kg}$ TCB (A), and with $5.0 \mathrm{mg} / \mathrm{kg} \mathrm{TCB} \mathrm{(B).} \mathrm{X-axis} \mathrm{is} \mathrm{number} \mathrm{of} \mathrm{days}$ subsequent to TCB injection. Y-axis is TCB concentration in $\mu \mathrm{g}$ TCB/g tissue lipid weight. Each point is a measurement on a single sample, consisting of pooled liver or muscle sections from all 4 or 5 fish in each treatment group. Plot symbols are: closed circle, liver sample, open circle, muscle sample. 


\section{Discussion}

In the present study we demonstrate that the Ah-receptor agonist 3,3',4,4'-TCB induces cellular alterations in several organs of the marine fish scup. TCB primarily targets epithelial tissues (hepatocytes, renal tubules, gill epithelia) and lymphoid tissues (renal hematopoietic cells, spleen) in scup. Similarly, epithelial and lymphoid tissues were found to be primary targets of histopathology induced by the Ah-receptor agonist TCDD in rainbow trout and perch (Spitsbergen et al., 1988a; Spitsbergen et al., 1988b; van der Weiden et al., 1992). Studies in mammals indicate that TCDD and the structurally related halogenated biphenyls cause alterations in the proliferation and differentiation of epithelial cells (e.g. chloracne), and cause histopathology in lymphoid organs (e.g. thymic atrophy) (Goldstein and Safe, 1989). Structure-activity studies in mammals suggest that these effects on epithelial and lymphoid tissues may be associated with ligand binding to the Ah receptor (Goldstein and Safe, 1989). Ah receptor has been identified in scup and other teleost fish (Hahn et al., 1994), thus some of the tissue damage found in the present study and others may be related to, or mediated by, TCB binding to this receptor.

Liver We attribute the TCB-induced basophilia of hepatocytes in the present study to a proliferation of endoplasmic reticulum in liver, and to a loss of lipid and glycogen stores. TCDD treatment has previously been associated with decreased hepatocyte glycogen in rainbow trout, perch, and lake trout sac fry (Spitsbergen et al., 1988a; Spitsbergen et al., 1988b; Spitsbergen et al., 1991). Treatment with a high dose of Aroclor has been found to elicit an increased content of smooth endoplasmic reticulum in channel catfish hepatocytes, which was directly correlated with increases in hepatic microsomal monooxygenase (P450) activity (Klaunig et al., 1979). Similarly, TCB treatment induced the hepatic content of cytochrome P450 in these scup (chapter 2), concurrently with the apparent increases in hepatic endoplasmic reticulum content. Such proliferation of endoplasmic reticulum may be prerequisite to the induction of microsomal cytochromes $\mathrm{P} 450$, which are integral membrane proteins in the endoplasmic reticulum.

Spleen We report a consistently greater number of melanomacrophage centers in spleen of TCB-treated fish as compared to vehicle-treated fish (Table 1). Factors known to increase melanomacrophage aggregates include chronic disease, chemical exposure, infectious disease, old age, and starvation (Ferguson, 1989). In such cases, the melanomacrophage centers act to sequester particulate material, including cellular breakdown products (Ferguson, 1989). Hence, we speculate that this histological alteration served a protective, or "clean-up", function in TCB-exposed fish. 
Kidney TCB treatment caused a severe degeneration of renal tubules in scup exposed to either dose. By day 12 foci of regeneration were evident, but epithelial necrosis was ongoing. We found no reports of similar symptoms in fish treated with PCBs or dioxins, however, Rao and Banerji (1990) report a very similar degeneration of proximal and distal tubules in the kidney of rat fed Aroclor. The renal tubular epithelium is a major route for the excretion of foreign compounds in fish (Ferguson, 1989), thus severe tubular damage may be expected to lead to impaired excretory capacity. Plasma chemistry (Table 2), conducted on a separate group of fish treated with either vehicle or $0.1 \mathrm{mg} / \mathrm{kg} \mathrm{TCB}$, indicated that TCB treatment caused no significant change in plasma content of electrolytes or nitrogenous waste products. Thus excretory capacity, indicative of both kidney and gill function, was apparently not compromised in a group of fish administered $0.1 \mathrm{mg} / \mathrm{kg} \mathrm{TCB}$.

Gill TCB induced hyperplasia of gill epithelia in half of the fish treated with 0.1 $\mathrm{mg} / \mathrm{kg}$ and all of the fish treated with $5.0 \mathrm{mg} / \mathrm{kg}$, on days 12 and 16 after treatment. At the high dose some fish displayed diffuse hyperplasia, causing fusion of secondary lamellae on most of the primary gill filaments, and thus drastically reducing the gill surface area. There was no evidence of gill hyperplasia in vehicle-treated fish, with the exception of a single animal sampled on day 12, that received a score of 1 (see Methods). Epithelial hyperplasia is believed to be a general stress response and is induced in fish gill by a wide variety of toxicants and parasites (Evans, 1987). Gill epithelia play a critical role in gas exchange, maintenance of acid/base balance, ionic regulation, and excretion of nitrogenous waste (Ferguson, 1989). Epithelial hyperplasia could reduce the effective surface area available for these functions, and could increase the diffusional distances from water to blood (within the gill pillar endothelia). For example, the induction of epithelial hyperplasia by chlorine exposure was seen to correlate with reduced oxygen uptake efficiency in trout (Black and McCarthy, 1990).

Thus a greatly reduced epithelial surface area, and greatly increased diffusional distances, in gills of scup treated with $5.0 \mathrm{mg} / \mathrm{kg}$ TCB could have elicited a range of effects, from inhibited respiration to impaired waste excretion. Unlike the kidney pathology in the present study, which was very similar at the two TCB doses until day 12 , gill pathology was consistently more extensive in fish treated with the high TCB dose. We therefore speculate that impaired gill function may have been a major contributing factor in the death of fish treated with $5.0 \mathrm{mg} / \mathrm{kg}$ TCB. Necropsies were conducted on gills of two fish that died spontaneously, and these two animals received scores of 3 and 4 , respectively, for epithelial hyperplasia (see Methods), confirming that the moribund fish had severe gill lesions. 
Tail fin Fin erosion in fish is associated with a wide variety of environmental stressors, including lipophilic organic compounds, heavy metals, waste water, crude oil, pathogens (reviewed in Sindermann, 1979), and nutrient depletion. Fin erosion has long been known to be associated with environmental PCB contamination (McDermott-Ehrlich et al., 1977; Sherwood and Mearns, 1977), and has been induced in numerous studies by prolonged exposure to TCDD (among the earliest, Miller et al., 1973; Hawkes and Norris, 1977)). Literature reports of histopathology associated with fin erosion describe principally epithelial lesions. Among the earlier reports, Murchelano (1975) described epidermal hyperplasia, dermal fibrosis, hyperemia, and hemorrhage, without an inflammatory response, in winter flounder from a contaminated environment. Wellings et al. (1976) reported epidermal hyperplasia, dermal fibrosis, focal necrosis of epidermis, an inflammatory response, and in some cases an apparent resorption of the bony fin rays, in flounder and sole from a contaminated environment. In the present study we describe epithelial lesions and inflammation, as have been noted by other investigators, and we also report an unusual lesion in bone tissue associated with fin erosion. The lesions in bone were likely secondary to those in epithelia, as they only occurred in areas of the fin where the protective soft tissues were eroded away.

The mechanisms by which PCB and dioxin induce fin erosion are not fully known. These compounds may be acting as immune suppressants, rendering superficial tissues of treated fish more susceptible to attack by water-borne pathogens. However, it cannot be ruled out that PCBs or dioxins may exert a direct effect on epithelial or bone cells, or that Ah receptor binding of these compounds may mediate these tissue alterations.

TCB residues Among the fish treated with $0.1 \mathrm{mg} / \mathrm{kg}$ TCB, lesions and cellular alterations were less severe in the group sampled on day 16 than in the group sampled on day 12. On day 16 , the kidneys displayed no tubule necrosis and had abundant hematopoietic tissue, the number of macrophage aggregates in spleen was closer to control levels, the mean score for liver basophilia was lower than that on day 12 , and the mean score for gill hyperplasia was also lower than that on day 12 . TCB residue analysis on pooled liver samples indicated that TCB content was maximal on day 5 in fish treated with $0.1 \mathrm{mg} / \mathrm{kg}$, and was maximal on day 12 in fish treated with $5.0 \mathrm{mg} / \mathrm{kg}$ (Fig 6). Depuration of TCB was apparent by day 12 in fish treated with $0.1 \mathrm{mg} / \mathrm{kg}$, and by day 16 in fish treated with $5.0 \mathrm{mg} / \mathrm{kg}$. It is possible that in fish treated with the low TCB dose, repair of tissue lesions occurred from day 5 (the apparent onset of depuration) to day 16 (the final sampling point), suggesting that tissues are capable of returning toward normal morphology within a short time after of toxicant elimination. Most lesions appeared to be persistent in fish treated with $5.0 \mathrm{mg} / \mathrm{kg} \mathrm{TCB}$, consistent with the high levels of TCB 
remaining in tissues on day 16 , even after the bulk of the dose had been depurated (Table $3)$.

In conclusion, we report that treatment of a marine teleost with $3,3^{\prime}, 4,4^{\prime}-\mathrm{TCB}$ is associated with lesions in several epithelial tissues, including hepatocytes, renal tubules, gill epithelia, and superficial epithelia of tail fin. We also report a depletion of lymphoid tissues, and either an increase or a rearrangement of melanomacrophage cells in kidney and spleen. Most of the histological alterations in liver, spleen, kidney, and gill were elicited by both $0.1 \mathrm{mg} / \mathrm{kg} \mathrm{TCB}$ and $5.0 \mathrm{mg} / \mathrm{kg} \mathrm{TCB}$, and we provide suggestive evidence that fish treated with the lower dose may have achieved full recovery from many of the alterations following TCB depuration. Histopathology in epithelial and lymphoid tissues may be downstream consequences of TCB binding to the cytosolic Ah receptor, and may also be related to microsomal interactions between $\mathrm{TCB}$ and CYP1A1, including the possible in situ production of reactive TCB metabolites and reactive oxygen species. 


\section{CHAPTER FIVE}

\section{In vitro Metabolism of Polychlorinated Biphenyl (PCB)}

Congeners by the Marine Fish Scup (Stenotomus chrysops):

Relationship to Cytochrome P450 Expression 


\begin{abstract}
Marine fish are exposed to high concentrations of polychlorinated biphenyl (PCB) contaminants in coastal areas worldwide. However, there is little direct information concerning the mechanisms and capacity for PCB metabolism by teleost species. We analyzed and compared in vitro metabolism of the tetrachlorobiphenyl (TCB) congeners 3,3',4,4'-TCB (IUPAC \#77) and 2,2',5,5'-TCB (IUPAC \#52) in the marine fish scup
\end{abstract} (Stenotomus chrysops). Metabolism rates were quantified using a radiometric assay, in which production of aqueous-soluble TCB derivatives was measured. Hepatic microsomes from feral scup metabolized 3,3',4,4'-TCB and 2,2',5,5'-TCB at approximate rates of 0.13 and $2.06 \mathrm{pmol} / \mathrm{min} / \mathrm{mg}$ microsomal protein, respectively. 3,3',4,4'-TCB turnover was inhibitable by cytochrome $\mathrm{c}, \mathrm{CO}$, and $\alpha$-naphthoflavone, indicating that it was a $\mathrm{P} 450$ catalyzed activity. Liquid chromatography of reaction products on XAD resin resolved one water-soluble metabolite peak and two methanol-soluble metabolite peaks from each congener. Treatment of depurated fish with the CYP1A inducers $\beta$-naphthoflavone, 3methylcholanthrene, and 2,3,7,8-tetrachlorodibenzofuran caused moderate increases in the rate of metabolism of 3,3',4,4'-TCB, correlated with increases in microsomal CYP1A1 content. Fish treated with CYP1A inducers also had slightly increased rates of 2,2',5,5'TCB metabolism. However, among the treatment groups there was a strong positive correlation between rate of metabolism of 2,2',5,5'-TCB and microsomal content of the putative teleost CYP2B. Feral fish had significantly greater rates of metabolism of $2,2^{\prime}, 5,5^{\prime}$-TCB than did depurated or depurated/treated fish, which corresponded to an elevated content of microsomal CYP2B. In competitive inhibition studies, neither TCB congener inhibited the metabolism of the other one, suggesting two separate principal catalysts. 3,3',4,4'-TCB strongly inhibited both EROD and MROD activity while 2,2',5,5'-TCB inhibited neither one; neither congener inhibited PROD activity. We conclude that scup have the capacity to transform PCBs to aqueous-soluble derivatives in vitro, which suggests in vivo production of excretion products. Induction and inhibition studies suggest that scup CYP1A1 is a principal catalyst for the metabolism of 3,3',4,4'TCB, while a putative scup CYP2B homologue is a principal catalyst for the metabolism of $2,2^{\prime}, 5,5^{\prime}-\mathrm{TCB}$ in scup liver microsomes, as is the case in rat liver microsomes. The metabolism of $2,2^{\prime}, 5,5^{\prime}-\mathrm{TCB}$ is more rapid than that of 3,3',4,4'-TCB, which lacks adjacent, unsubstituted meta-para positions. However, in vitro rates with either congener are quite slow. The basis for slow turnover of substrates that bind well to the active site is unknown. 


\section{Introduction}

Polychlorinated biphenyls (PCB) are ubiquitous contaminants in the marine environment (Tanabe, 1988; Fowler, 1990), having been discharged into coastal waters during several decades of industrial use. Due to their chemical stability and hydrophobicity, PCBs have tended to concentrate in marine sediments (Tanabe, 1988; Fowler, 1990) and have entered the food chains of teleost fish. The fates of lipophilic organic compounds in fish include long-term retention in fatty tissues, elimination of parent compound via the gills, urine, or bile, elimination in gametes at spawning, and biotransformation to metabolites. There is a large body of work concerning PCB uptake and elimination in fish, but little is known about metabolism of PCBs by fish. In the earliest studies, some investigators found no evidence for in vivo metabolism of PCB congeners in fish (Hutzinger et al., 1972; Narbonne, 1979). Others detected metabolites at very low levels in bile or in whole organisms (Metcalf et al., 1975; Sanborn et al., 1975; Melancon and Lech, 1976; Hinz and Matsumura, 1977; Herbst et al., 1978; Varanasi et al., 1987). These early studies dealt primarily with non-coplanar congeners such as 2,2 ',5,5'TCB; those studies that did investigate coplanar PCB congeners found no metabolism of 4-MCB and 4,4'-DCB; (Hutzinger et al., 1972; Herbst et al., 1978). More recently, some investigators have inferred PCB metabolism in fish, having observed selective loss of certain congeners in analysis of PCB residues in tissue (Niimi and Oliver, 1983; Boon et al., 1989; Brown, 1992; de Boer et al., 1993). There is no direct information concerning the specific catalysts involved in PCB metabolism in fish, their rates of metabolic activity, and their regulation. Such information is important to our understanding of factors that may affect PCB congener metabolism, and thus determine the effects of PCBs, in fish.

In mammals, the oxidative metabolism of PCBs is catalyzed primarily by cytochromes $\mathrm{P} 450$, which play a role in both detoxification and activation of substrates. In detoxification, P450-generated metabolites may be further metabolized by secondary enzymes to nontoxic, aqueous-soluble products that are readily excreted. Alternatively, oxygenated metabolites may be unstable and reactive, and may form covalent attachments with biomolecules. The formation of both innocuous and active metabolites can depend upon the level of expression and activity of specific cytochromes P450, and of secondary metabolizing enzymes, in target tissues.

Prior work in mammals indicates that structure-activity relationships govern the metabolism of PCBs by cytochromes $\mathrm{P} 450$, with chlorine position determining which $\mathrm{P} 450$ enzyme carries out the transformation. In rat liver, hydrocarbon-inducible CYP1A forms metabolize congeners with halogenated substituents at one or both para positions, 


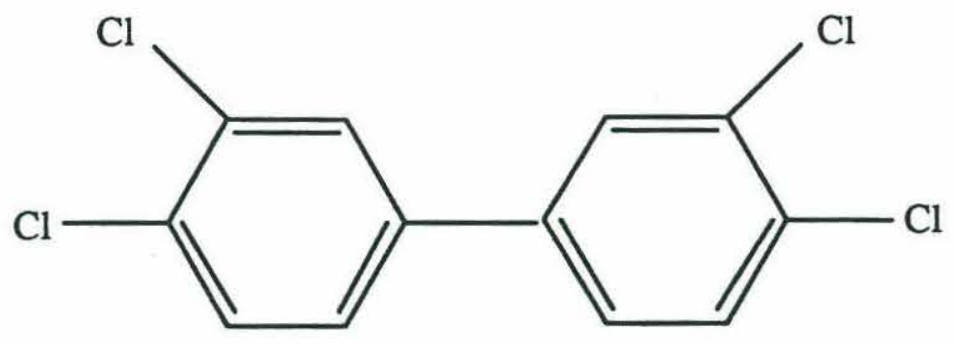

3, 3', 4, 4'-TCB

\section{Congener 77}

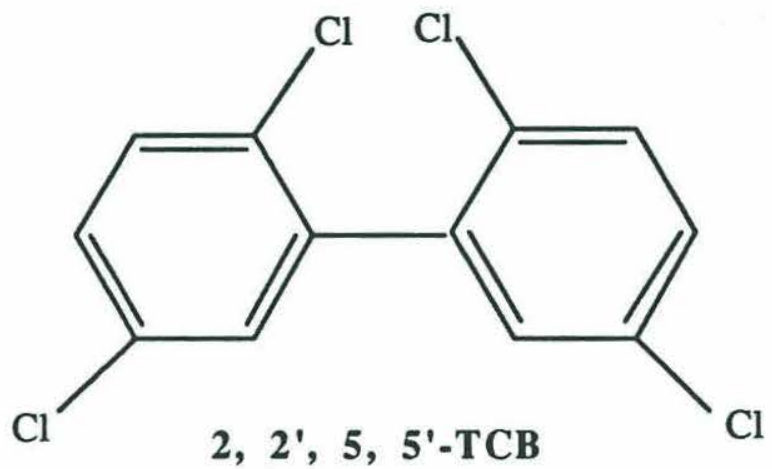

Congener $\mathbf{5 2}$

Figure 1. Polychlorinated biphenyl congeners used in the study: 3,3',4,4'-TCB (IUPAC \#77); 2,2',5,5'-TCB (IUPAC \#52). 
and with adjacent unsubstituted ortho-meta carbons, while phenobarbital-inducible CYP2B forms (CYP2B1, CYP2B2) metabolize congeners with adjacent, unsubstituted meta-para positions (Kaminsky et al., 1981; Kennedy et al., 1981; Shimada and Sawabe, 1983; Mills et al., 1985; Ishida et al., 1991). In addition, it was observed that PCB congeners with two or more ortho substituents are more rapidly metabolized by rat CYP2B than by CYP1A, while congeners lacking ortho substituents are more rapidly metabolized by rat CYP1A than by CYP2B.

There is currently no direct information concerning the role of cytochromes P450 in PCB metabolism in fish. One may deduce from the literature the involvement of CYP1Alike and CYP2B-like activity, based on the relative abundance of PCB congeners found in fish tissue (Niimi and Oliver, 1983; Boon et al., 1989; Brown, 1992; de Boer et al., 1993). Here we report on the metabolism of two tetrachlorobiphenyl (TCB) congeners by hepatic microsomes of the marine fish scup (Stenotomus chrysops): 3,3',4,4'-TCB (IUPAC \#77), a rat CYP1A substrate and an Ah receptor agonist, and 2,2',5,5'-TCB (IUPAC \# 52), a substrate and inducer of the phenobarbital-inducible rat CYP2B forms (Fig 1). We find that scup metabolize both congeners to aqueous-soluble products in vitro. We present evidence to indicate that TCB metabolism in fish is accomplished by cytochrome P450, and that metabolism of the two congeners is by different P450s. Metabolism of 3,3',4,4'-TCB is catalyzed by the scup CYP1A1 homologue and metabolism of 2,2',5,5'-TCB appears to be catalyzed by a scup CYP2B-like protein, thus following the same substrate SARs that have been observed in rat.

\section{Materials and Methods}

Chemicals 3,3',4,4'-TCB [U-14 C] $(52.1 \mathrm{mCi} / \mathrm{mmol})$ was purchased from Pathfinder (St. Louis, MO). Analysis by GC/ECD and GC/MS revealed no hydrophobic contaminant peaks ( $>99 \%$ pure); small polar contaminants were completely removed by dissolving the TCB in hexane and extracting twice with $\mathrm{NaOH}$. 2,2',5,5'-TCB [U-14 $\mathrm{C}$ ] $(14.2$ $\mathrm{mCi} / \mathrm{mmol}$ ) was purchased from Sigma (St. Louis, MO). This product was determined to be $>98 \%$ pure by HPLC analysis, conducted on an ODS- 2 column by the manufacturer. Unlabeled 3,3',4,4'-TCB was obtained from Pathfinder (St. Louis, MO). Purity of the TCB was $>99 \%$ by GC/ECD and GC/MS analysis. Unlabeled 2,2',5,5'-TCB was obtained from Ultrascientific (North Kingstown, RI). Manufacturer analysis by GC/FID indicated $>99 \%$ purity. Ethoxyresorufin, methoxyresorufin, and pentoxyresorufin were from Molecular Probes (Eugene, OR). All other chemicals used were of the highest grade available from commercial suppliers. 
Animals Scup (Stenotomus chrysops) were caught by angling in Tarpaulin Cove and Buzzards Bay, Massachusetts. Some individuals were killed immediately upon capture, and livers were stored in liquid nitrogen until microsomes were prepared. Prior studies in our laboratory suggest that enzyme degradation does not occur to any appreciable degree in scup tissues stored at liquid nitrogen temperatures (unpublished observations). Livers from these individuals were pooled for microsome preparation (approximately 20 to 70 fish per pool) and used for quantification of parameters in "feral" fish. Other individuals were held in laboratory aquaria for one year to allow depuration of environmental residues. Depurated fish were treated by intraperitoneal injection with either corn oil ("control" fish), $\beta$-naphthoflavone (BNF; $20 \mathrm{mg} / \mathrm{kg}$ ), 3-methylcholanthrene (MC; $20 \mathrm{mg} / \mathrm{kg}$ ), or 2,3,7,8tetrachlorodibenzofuran (TCDF; $3 \mu \mathrm{g} / \mathrm{kg}$ ), and killed five days later by cervical transection. All treatments were not carried out at the same time, thus corn oil-treated fish are not strictly "controls" for each of the treatments. Nonetheless, extensive work in our laboratory has shown that depurated, corn oil-treated scup express cytochrome P450 parameters at predictably low levels. Livers from all injected fish were removed immediately upon killing, and microsomes were prepared by differential centrifugation, as previously described (Hahn and Stegeman, 1994). Microsomal protein content was measured with the bicinchoninic acid method (Smith et al., 1985), using bovine serum albumin as a standard.

Hepatic microsomes from rat were obtained from Dr. Mark Melancon (U.S. Fish and Wildlife Service, Laurel, MD). Fisher-344 rats were treated by intraperitoneal injection with either corn oil (control, day 1), 3-methylcholanthrene in corn oil (MC, $20 \mathrm{mg} / \mathrm{kg}$, day 1 ), or phenobarbital in saline ( $\mathrm{PB}, 80 \mathrm{mg} / \mathrm{kg}$, days $1,2,3)$. Animals were killed on day four by decapitation, and microsomes were prepared by differential centrifugation (Melancon et al., 1990).

Characterization of monooxygenase systems The content of microsomal cytochrome P450 was determined using dithionite difference spectra of CO-treated samples, as previously described (Stegeman and Binder, 1979), with an extinction coefficient of $91 \mathrm{mM}^{-1} \mathrm{~cm}^{-1}$ (Omura and Sato, 1964). Ethoxyresorufin O-deethylase (EROD) activity was quantified at $32^{\circ} \mathrm{C}$ with a fluorometric kinetic assay, using a Cytofluor 2300 fluorescence plate reader (Millipore), as described previously (Hahn et al., 1993).

Immunoblot analysis was performed as previously described (Kloepper-Sams et al., 1987; Stegeman et al., 1991). Primary antibodies used were (1) monoclonal antibody 1-12-3, raised against scup cytochrome P450E (CYP1A1) (Park et al., 1986), and (2) polyclonal antibody 7-94, raised against scup cytochrome P450B (Gray, 1988). 
Immunochemical cross-reactivity with rat CYP2B1 suggests that scup P450B is a CYP2B form (Stegeman et al., 1990a; Stegeman et al., 1990b). Secondary antibodies used were (1) alkaline phosphatase linked goat-anti-mouse IgG, and (2) alkaline phosphatase linked goat-anti-rabbit IgG. Content of each enzyme in hepatic microsomes was quantified by densitometric scanning of antibody-reactive bands with a video imaging system (Masterscan, Scanalytics, division of CSPI, Billerica, MA), and comparison to P450E and P450B standards.

TCB/P450 binding spectrum Hepatic microsomes from feral scup were diluted to a concentration of $1 \mathrm{mg} / \mathrm{ml}$ in a Tris buffer (see below), and divided evenly between two cuvettes. 3,3',4,4'-TCB was dissolved in DMSO to a concentration of $0.5 \mathrm{mM}$, and added to the microsomal suspension in the sample cuvette at concentrations ranging from $1 \mu \mathrm{M}$ to $10 \mu \mathrm{M}$ (final concentration of $0.2 \%$ to $2 \% \mathrm{DMSO}$ ). Equivalent volumes of DMSO were added to the reference cuvette. A uv/visible difference spectrum of the microsome/TCB suspension was obtained by scanning over a wavelength range of $350 \mathrm{~nm}$ to $500 \mathrm{~nm}$ on a Shimadzu UV-260 spectrophotometer.

TCB metabolism assay The assay of Schnellman et al. (1983) was used, with modifications, to assess metabolism of 3,3',4,4'-TCB and 2,2',5,5'-TCB. Incubations were performed at $30^{\circ} \mathrm{C}$ under air in a shaking incubator. This temperature was determined to be optimal for CYP1A1 activity in scup hepatic microsomes (Stegeman et al., 1979). Incubations were $0.5 \mathrm{ml}$ and contained $1 \mathrm{mg} / \mathrm{ml}$ microsomal protein (unless stated otherwise), $50 \mathrm{mM}$ Tris, $\mathrm{pH}$ 7.6, $0.1 \mathrm{mM}$ EDTA, and $2 \mu \mathrm{M}$ or $5 \mu \mathrm{M}$ (nominal concentration) ${ }^{14} \mathrm{C}$-labeled TCB that had been dissolved in acetone (acetone concentration in the incubations was approximately $1 \%$ to $3 \%$ ). Substrate saturation profiles were obtained to determine that the $2 \mu \mathrm{M}$ TCB and $5 \mu \mathrm{M}$ concentrations were optimal in scup; both concentrations gave the same rate of metabolism in vitro. For assays conducted with rat liver microsomes, incubations were performed at $37^{\circ} \mathrm{C}$ using $5 \mu \mathrm{M}{ }^{14} \mathrm{C}$-labeled TCB (6\% acetone in the incubation). After a 2 minute pre-incubation, reactions were initiated by the addition of $1 \mathrm{mM}$ NADPH and run for 60 minutes (unless stated otherwise). Reactions were terminated by the addition of $0.75 \mathrm{ml} 0.5 \mathrm{~N} \mathrm{NaOH}$, and heated at $70^{\circ} \mathrm{C}$ for 10 minutes to aid in microsome solubilization (Schnellman et al., 1983). To remove parent $\mathrm{TCB}$, the samples were extracted two to three times with $2.5 \mathrm{ml}$ aliquots of $\mathrm{n}$-hexane : $100 \%$ ethanol (19:1). $500 \mu \mathrm{l}$ of the aqueous/ $\mathrm{NaOH}$ sample was then neutralized with $\mathrm{HCl}$ and combined with $6 \mathrm{ml}$ Scintiverse II (Fisher Scientific), and aqueous radioactivity was quantified by measuring ${ }^{14} \mathrm{C}$ with liquid scintillation counting on a Beckman LS5000TD 
counter. The rate of production of aqueous-soluble metabolites was determined by subtracting the aqueous radioactivity in control incubations, containing either boiled microsomes or no microsomes (both controls gave similar results), and dividing by specific activity of the substrate to convert dpm (disintegrations per minute) to pmol PCB metabolites. The limits of resolution of the assay were approximately $20 \mathrm{dpm}$, giving approximate detection limits of $0.03 \mathrm{pmol} / \mathrm{min} / \mathrm{mg}$ microsomal protein for 3,3',4,4'-TCB metabolites, and $0.1 \mathrm{pmol} / \mathrm{min} / \mathrm{mg}$ for 2,2',5,5'-TCB metabolites. Duplicate, and in some cases triplicate, assays were performed on each sample.

Some metabolism assays were conducted in controlled atmospheres rather than under air, according to Binder (1982) with modifications. In these reactions, microsomes, buffer, and substrate were combined and the mixture was bubbled gently with either $\mathrm{N}_{2}$ or $\mathrm{CO}$ for 60 seconds. Tubes were immediately sealed with air-tight serum stoppers. The gas phase in the tube above the reaction mixture, either $\mathrm{N}_{2}$ or $\mathrm{CO}: \mathrm{O}_{2}(80: 20)$, was then prepared as follows. The sealed volume of each tube was connected to a manifold with capillary tubing, and a syringe needle attached to the capillary tubing was passed through the serum stopper. The tube-syringe units were sequentially evacuated and then gassed with either $\mathrm{N}_{2}$ or $\mathrm{CO}$. After several cycles, ending with the gassing, the tubes were disconnected from the manifold. In the $\mathrm{CO}$ tubes, the total volume of gas was adjusted to 8 $\mathrm{ml}$ with a syringe, and $2 \mathrm{ml}$ of $\mathrm{O}_{2}$ was injected through the serum stopper. Reactions were initiated by injection of NADPH through the serum stoppers with a syringe, and run as above.

XAD column chromatography Aqueous-soluble metabolites of ${ }^{14} \mathrm{C}$-labeled TCBs were generated by hepatic microsomes of feral scup (3,3',4,4'-TCB and 2,2',5,5'-TCB), MCtreated rat $\left(3,3^{\prime}, 4,4^{\prime}-\mathrm{TCB}\right)$, and control rat $\left(2,2^{\prime}, 5,5^{\prime}-\mathrm{TCB}\right)$, as above. Incubations were extracted with hexane/ethanol as above, and the remaining aqueous/ $\mathrm{NaOH}$ sample was applied to the column. XAD columns were prepared by pouring $5 \mathrm{~cm}$ of dry XAD-2 (coarse) resin (Amberlite polystyrene beads, Sigma) into a glass pasteur pipette, with a glass wool plug at the bottom. The column was pre-washed with several milliliters each of water, methanol, acetone, and water again, before sample application. Sample was applied and the column was sequentially washed with several milliliters each of water, methanol, and acetone (which elutes parent compound). Each $1 \mathrm{ml}$ fraction was collected and dried in a $60^{\circ} \mathrm{C}$ oven to reduce solvent volume. $5 \mathrm{ml}$ of Scintiverse II was added to each sample, and metabolites were quantified by liquid scintillation counting as above. 
Inhibition of TCB metabolism TCB metabolism assays were conducted as above, with several modifications. In some assays $1 \mathrm{mM} \mathrm{NADH}$ was substituted for NADPH in the assay buffer. Horse heart cytochrome c (Sigma) was dissolved in assay buffer and added to some assays at $100 \mu \mathrm{M} . \alpha$-naphthoflavone (ANF) dissolved in methanol was added to assays at $1 \mu \mathrm{M}, 10 \mu \mathrm{M}$, and $100 \mu \mathrm{M}(10 \mu \mathrm{l}$ methanol per $500 \mu \mathrm{l}$ assay); control and blank assays for comparison included $10 \mu \mathrm{l}$ methanol alone. Some assays were conducted with a single ${ }^{14} \mathrm{C}$-labeled TCB congener as the substrate, and with the other TCB congener added as an unlabeled "competitor". These assays included $2 \mu \mathrm{M}{ }^{14} \mathrm{C}$-labeled substrate (dissolved in $6 \mu \mathrm{l}$ acetone) and $4 \mu \mathrm{M}$ cold competitor (dissolved in $4 \mu \mathrm{l}$ acetone). To blank and control assays (those not containing cold competitor), $4 \mu \mathrm{l}$ acetone was added to balance the solvent volumes.

Inhibition of alkoxyresorufin O-dealkylase activities Ethoxyresorufin O-deethylase (EROD) activity, methoxyresorufin O-demethylase (MROD) activity, and pentoxyresorufin O-depentylase (PROD) activity were assayed by measuring the appearance of resorufin, using a Cytofluor 2300 fluorescence plate reader, as above. Substrate concentrations in the assays were $2 \mu \mathrm{M} \mathrm{ER}, 2 \mu \mathrm{M}$ MR, and $5 \mu \mathrm{M}$ PR (optimal concentrations). Microsomal protein concentrations in the assays were $0.16 \mu \mathrm{g} / \mu \mathrm{l}$ for EROD $(1 \mu \mathrm{l}$ microsomes per assay), $0.31 \mu \mathrm{g} / \mu \mathrm{l}$ for MROD ( $2 \mu \mathrm{l}$ microsomes per assay), and $1.6 \mu \mathrm{g} / \mu \mathrm{l}$ for PROD (10 $\mu \mathrm{l}$ microsomes per assay), to optimize detection. Each TCB congener was dissolved in acetone and added at concentrations of $0.25 \mu \mathrm{M}, 0.5 \mu \mathrm{M}, 0.75 \mu \mathrm{M}$, and $1.0 \mu \mathrm{M}(1 \mu \mathrm{l}$ acetone in $200 \mu \mathrm{l}$ assay). Control assays (without TCB) included $1 \mu \mathrm{l}$ acetone alone. Microsomes, buffer, substrate, and TCB were pre-incubated at $30^{\circ} \mathrm{C}$ for 5 minutes in a shaking water bath, and reactions were initiated by the addition of $1.1 \mu \mathrm{g} / \mu \mathrm{l} \mathrm{NADPH}$ (final concentration in assay).

Polyclonal antibody inhibition studies Metabolism of both TCB congeners was assayed in the presence of potentially inhibitory polyclonal antibodies. Assay conditions were as above, but incubations contained $5 \mu \mathrm{M}$ TCB in a total volume of $250 \mu \mathrm{l}$. Antibodies used were polyclonal 119p ("anti-CYP1A1"), which is a rabbit-anti-scup IgG raised against scup CYP1A1 (Kloepper-Sams et al., 1987); polyclonal 7-94 ("anti-CYP2B"), which is a rabbit-anti-scup IgG raised against scup P450B (Gray, 1988), the putative scup CYP2B (Stegeman et al., 1990a; Stegeman et al., 1990b); and c-93, which is the IgG fraction of non-immune rabbit serum, as a control. Prior studies have shown that polyclonal 119p binds to purified scup CYP1A1 in immunoblots and inhibits hepatic EROD activity and benzo[a]pyrene metabolism, whereas it does not bind to purified scup P450B in 
immunoblots and does not inhibit hepatic aminopyrine N-demethylase activity (KloepperSams et al., 1987) or hepatic testosterone 6 $\beta$-hydroxylase activity (unpublished observation). Unpublished studies in our laboratory have shown that polyclonal 7-94 binds to purified scup P450B in immunoblots but not to purified scup CYP1A1. Original antibody solutions were concentrated by centrifugation in Centricon 30 microconcentrators (Amicon, W.R. Grace and Co., Danvers, MA), and resuspended in $50 \mathrm{mM}$ Tris, $0.1 \mathrm{mM}$ EDTA, pH 7.6, at a concentration of $50 \mathrm{mg} / \mathrm{ml}$. Protein content was measured in the original antibody solution and in the Centricon filtrate fractions by monitoring absorbance at $280 \mathrm{~nm}$, to assure that only a very small fraction of antibody was lost during the preparation. Antibodies and serum were used in TCB metabolism assays at 15, 30, and 60 $\mu \mathrm{g} / \mathrm{pmol} \mathrm{P} 450$. The $250 \mu \mathrm{l}$ assay contained $90 \mathrm{pmol}$ total cytochrome P450 in $250 \mu \mathrm{g}$ microsomal protein, thus antibodies were added at $1350 \mu \mathrm{g}, 2700 \mu \mathrm{g}$, and $5400 \mu \mathrm{g}$, respectively. Microsomes, buffer, and antibody were combined in test tubes (116 $\mu$ l total volume) and pre-incubated for one hour on ice. Reactions were initiated by the addition of TCB, NADPH, and the remaining buffer volume needed, and carried out as above.

The same polyclonal antibodies were added to EROD activity assays. Antibodies and serum were used at $30 \mu \mathrm{g} / \mathrm{pmol} \mathrm{P} 450$ and $60 \mu \mathrm{g} / \mathrm{pmol} \mathrm{P} 450$. The $200 \mu \mathrm{l}$ assay contained $11.3 \mathrm{pmol}$ total cytochrome $\mathrm{P} 450$ in $31.5 \mu \mathrm{g}$ microsomal protein, thus antibodies were added at $339 \mu \mathrm{g}$ and $678 \mu \mathrm{g}$, respectively. Microsomes and antibody were preincubated in a small volume of buffer, for one hour on ice, prior to the addition of ethoxyresorufin substrate in additional buffer. Reactions were initiated by the addition of $\mathrm{NADPH}$, and run as above.

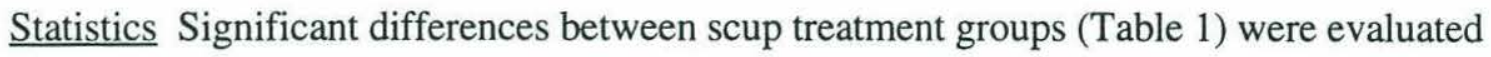
with a one-factor ANOVA test, using Statview SE+ Graphics, v.1.03 (Abacus Concepts) for Macintosh.

\section{Results}

\section{TCB metabolism}

Hepatic microsomes from freshly caught animals metabolized both 3,3',4,4'-TCB and 2,2 ',5,5'-TCB to aqueous-soluble metabolites in vitro, in a manner that was linear with incubation time and that increased with increasing content of microsomal protein (Fig 2). The apparent turnover of 3,3',4,4'-TCB to aqueous-soluble products was dependent on native protein and on NADPH cofactor (Fig 3), consistent with an enzyme-catalyzed reaction. NADH supported TCB metabolism at rates $60 \%$ less than with NADPH (Fig 3 ). 

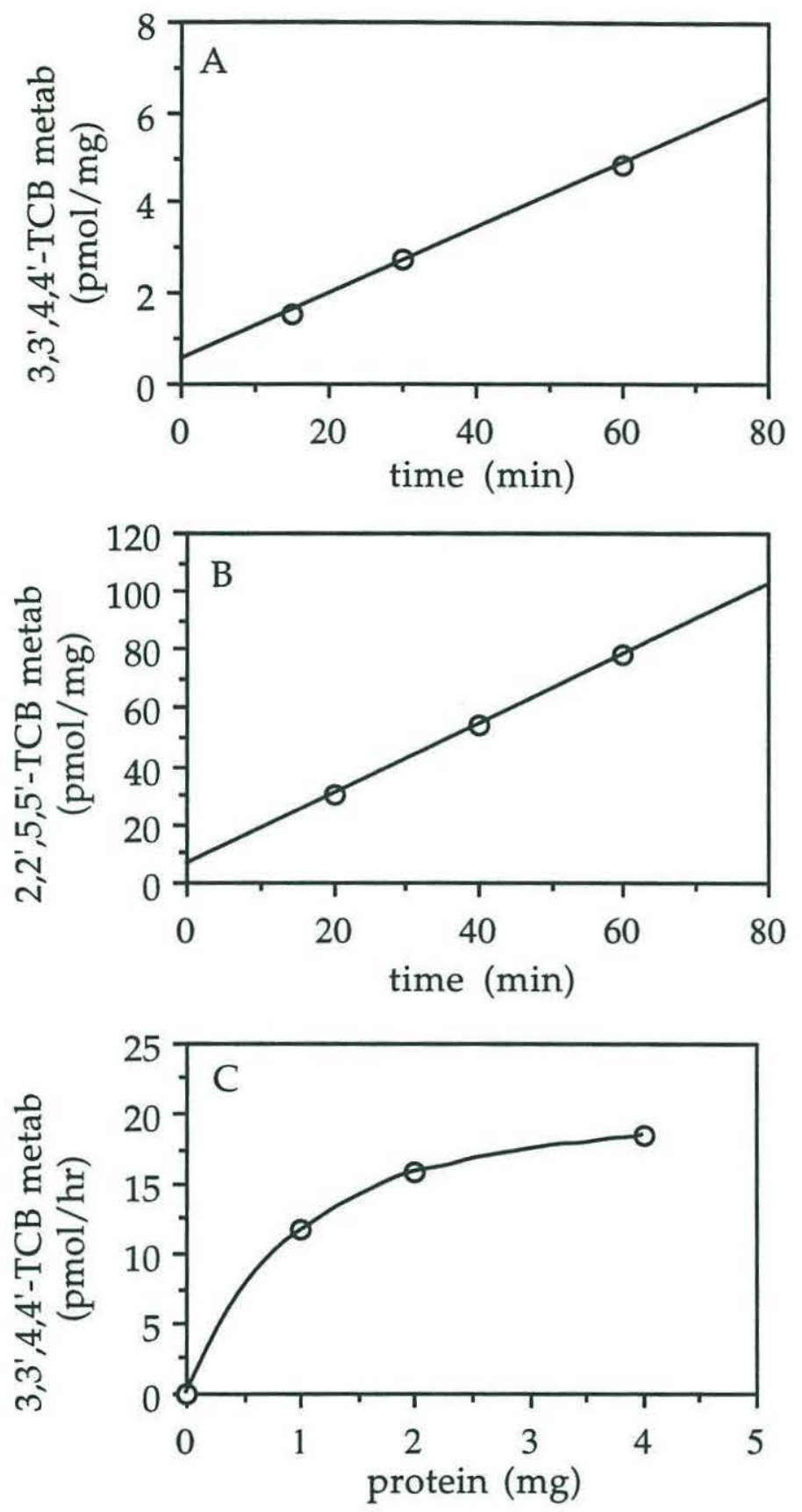

Figure 2. Metabolism of TCB congeners in hepatic microsomes of feral scup. Rates of TCB metabolism were assessed radiometrically, in $1 \mathrm{ml}$ incubations containing $1 \mathrm{mg} / \mathrm{ml}$ microsomal protein (A, B) and conducted for one hour (C). Each point is the mean of triplicate assays on a single, pooled microsomal sample. (A) Metabolism of 3,3',4,4'-TCB (pmol/mg) versus time of incubation (min). (B) Metabolism of 2,2',5,5'-TCB (pmol/mg) versus time of incubation (min). (C) Metabolism of 3,3',4,4'-TCB (pmol/hour) versus content of microsomal protein in the $1 \mathrm{ml}$ incubation $(\mathrm{mg})$. 


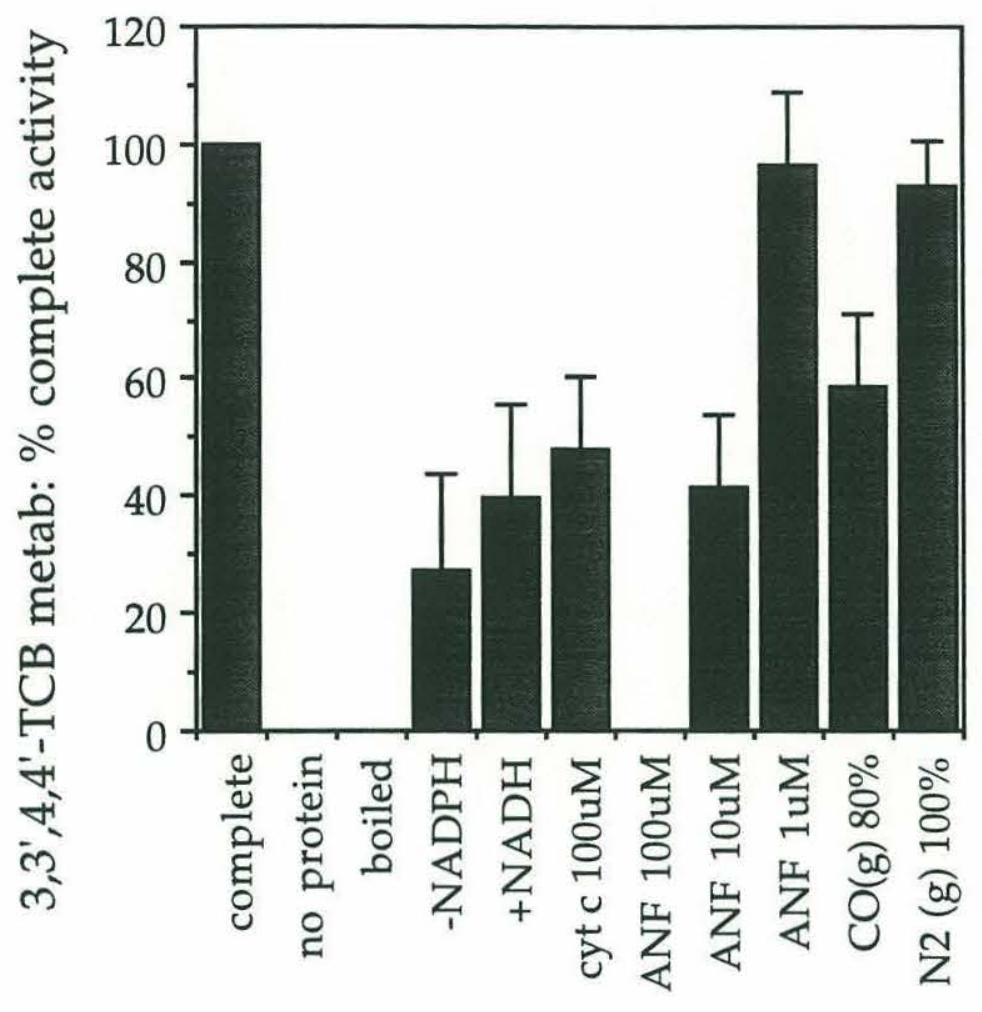

Figure 3. Inhibition of 3,3',4,4'-TCB metabolism in hepatic microsomes of feral scup. The farthest left bar represents the rate of metabolism in a complete incubation (100\%), assayed radiometrically as in Methods (actual rates were as listed in Table 1). Subsequent bars indicate \% complete activity in assays containing: no microsomes, boiled microsomes, no NADPH, NADH substituted for NADPH, $100 \mu \mathrm{M}$ cytochrome c, $100 \mu \mathrm{M}$ ANF, $10 \mu \mathrm{M}$ ANF, $1 \mu \mathrm{M}$ ANF, CO gas (80\% atmosphere), and $\mathrm{N}_{2}$ gas (near 100\% atmosphere). Assays were conducted as described in Methods. The aqueous radioactivity in assays containing boiled microsomes was equal to that in assays conducted with no microsomes; these were used as subtractive blank assays and assigned a rate of zero. 
Addition of $100 \mu \mathrm{M}$ cytochrome $\mathrm{c}$, which competes with P450 for electrons from NADPH cytochrome P450 reductase, reduced the activity rate by $52 \%$ (Fig 3). Addition of ANF, a competitive inhibitor of CYP1A1 in scup (Klotz et al., 1984), reduced the rate of TCB metabolism in a concentration-dependent manner, with no detectable activity at $100 \mu \mathrm{M}$ ANF (Fig 3). Addition of $\mathrm{CO}$ ( $80 \%$ atmosphere), which binds reversibly to the ferrous cytochrome P450 heme, reduced the rate of TCB metabolism by $42 \%$ (Fig 3). These data indicate that the primary metabolism of 3,3',4,4'-TCB in scup liver microsomes is catalyzed largely by cytochrome(s) P450.

In contrast, an $\mathrm{N}_{2}$ gas atmosphere, which should limit the availability of $\mathrm{O}_{2}$ for P450 catalysis, only inhibited TCB metabolism by $7 \%$ (Fig 3). Because the rate of metabolism of 3,3',4,4'-TCB is so slow (approximately $0.2 \mathrm{pmol} / \mathrm{min} / \mathrm{mg}$, maximum), very little $\mathrm{O}_{2}$ is required to maintain this activity in vitro. If one assumes a conservative catalytic efficiency of $10 \%$ (i.e. one turnover of TCB for every 10 turnovers of $\mathrm{O}_{2}$ by $\mathrm{P} 450)$, then only $120 \mathrm{pmol}\left(10^{-10}\right.$ moles) of $\mathrm{O}_{2}$ would be required to support this activity in one $\mathrm{mg}$ of microsomal protein for one hour. The $10 \mathrm{ml}$ volume of headspace in the assay tube prior to evacuation and gassing (see Methods) contains approximately $10^{-4}$ moles $\mathrm{O}_{2}$, based on the conversion factor of 0.209 liters $\mathrm{O}_{2}$ in one liter of air. It is not surprising that the methods used could not reduce the $\mathrm{O}_{2}$ concentration in the assay tube from $10^{-4}$ moles to $<10^{-10}$ moles; thus loss of activity in a (mostly) $\mathrm{N}_{2}$ atmosphere was not seen.

\section{Binding spectrum}

To further verify that the metabolism of 3,3',4,4'-TCB in scup hepatic microsomes involved a direct interaction with P450, binding spectra were obtained (Fig 4). The combination of TCB and microsomes produced a classic type I binding spectrum, with a peak at $390 \mathrm{~nm}$ and a trough at $420 \mathrm{~nm}$, indicative of the low spin to high spin transition of the ferric P450 associated with substrate binding to the active site. Due to the very small absorbance changes measured, we were unable to accurately determine a spectral dissociation constant from these data.

\section{Metabolite characterization}

Metabolites of ${ }^{14} \mathrm{C}-3,33^{\prime}, 4,4^{\prime}-\mathrm{TCB}$ and ${ }^{14} \mathrm{C}-2,2^{\prime}, 5,5^{\prime}-\mathrm{TCB}$ were generated in scup liver microsomes and separated with liquid chromatography on $\mathrm{XAD}$ resin. Microsomal incubations with 3,3',4,4'-TCB were carried out for 60 minutes, then parent compound was extracted with hexane:ethanol, and the remaining aqueous fraction was applied to the column. Three major peaks of ${ }^{14} \mathrm{C}$ eluted from the column: one in the first two $\mathrm{ml}$ of 


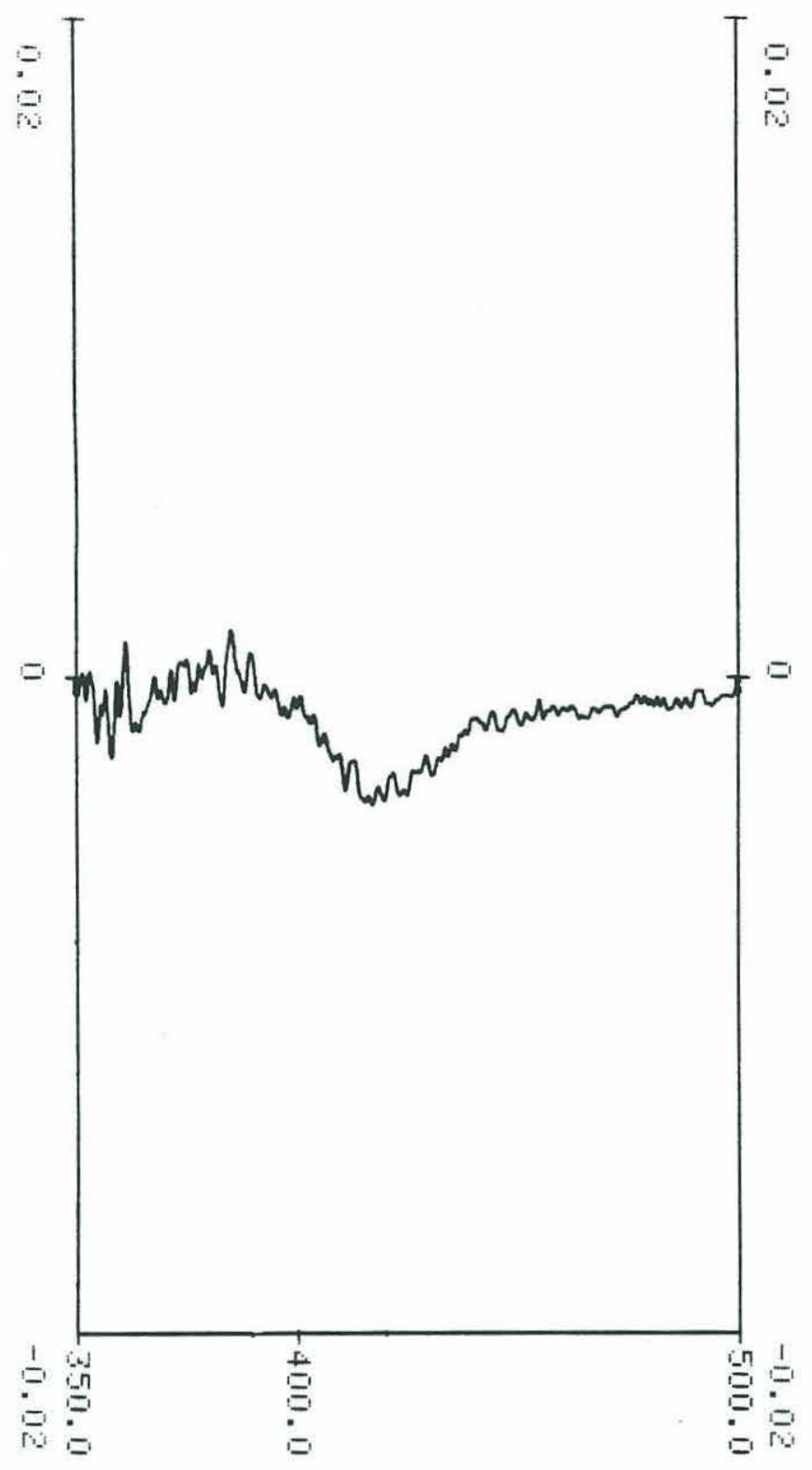

Figure 4. TCB / cytochrome P450 binding spectrum. $10 \mu \mathrm{M} 3,3^{\prime}, 4,4^{\prime}-\mathrm{TCB}$ was added to a $1 \mathrm{mg} / \mathrm{ml}$ suspension of hepatic microsomes from feral scup, and uv/visible difference spectrum was obtained. Plotted is absorbance $(y)$ versus wavelength $(x)$. TCB displays a typical type I binding spectrum, with a peak at $390 \mathrm{~nm}$ and a trough at $420 \mathrm{~nm}$. 
water, one in the first two $\mathrm{ml}$ of methanol, and one in the eleventh $\mathrm{ml}$ of methanol (Fig 5A and 5B). Very similar profiles were obtained with incubations using scup liver microsomes and microsomes from MC-treated rat (Fig 5), suggesting a similar formation of three classes of metabolites with different degrees of aqueous-solubility. When parent TCB alone was added to the column, it adhered to the matrix during water and methanol washes, and all radioactivity eluted in the first three acetone fractions (Fig 5C). We thus conclude that the water and methanol peaks are hydroxylated TCB derivatives generated during in vitro metabolism of ${ }^{14} \mathrm{C}$-labeled TCB.

Metabolites of 2,2',5,5'-TCB also eluted from XAD columns with water and methanol washes, following incubation in scup liver microsomes (Fig 6) or control rat liver microsomes (not shown). When parent 2,2',5,5'-TCB alone was added to the column, a major peak eluted in the first three acetone fractions. As with 3,3',4,4'-TCB, some microsomal incubations with 2,2',5,5'-TCB had small peaks eluting in acetone (Fig 6), which disappeared when the incubations were extracted once more with hexane:ethanol prior to application on the column. We thus attribute the latter peak to parent TCB. The ratio of water-soluble to methanol-soluble metabolites of 2,2',5,5'-TCB (measured as total, blank-subtracted, dpm of peak fractions eluting from XAD) increased with time of incubation (Fig 6). Thus, the proportion of metabolite radioactivity eluting in water washes increased from $38 \%$ (20 minutes) to $49 \%$ (40 minutes) to $57 \%$ (60 minutes). The proportion of total metabolites eluting in methanol decreased accordingly, as did the number of peaks eluting in methanol (Fig 6). We thus conclude that the metabolites eluting with water may be, at least in part, the product of further biotransformation of the metabolites eluting with methanol. The former peak could include dihydroxy TCBs and/or dihydrodiol TCBs, formed when mono-oxygenated products are attacked by cytochrome P450 and epoxide hydrolase, respectively.

\section{Induction of TCB metabolism}

Rates of in vitro metabolism of 3,3',4,4'-TCB and 2,2',5,5'-TCB were determined in liver microsomes from control scup (depurated and treated with corn oil), feral scup, and scup treated with various CYP1A inducers (Table 1). Levels of hepatic microsomal P450, immunodetectable CYP1A1 content, and rates of CYP1A1-catalyzed EROD activity were elevated in all groups as compared to control fish (Table 1). Immunodetectable content of the putative scup CYP2B was significantly greater in feral fish than in all other groups of fish analyzed, suggesting environmental exposure to compound(s) that elevate CYP2B (Table 1; see Discussion). The rate of metabolism of 3,3',4,4'-TCB in all groups of fish treated with CYP1A inducers tended to be greater than the rate in controls, although this 


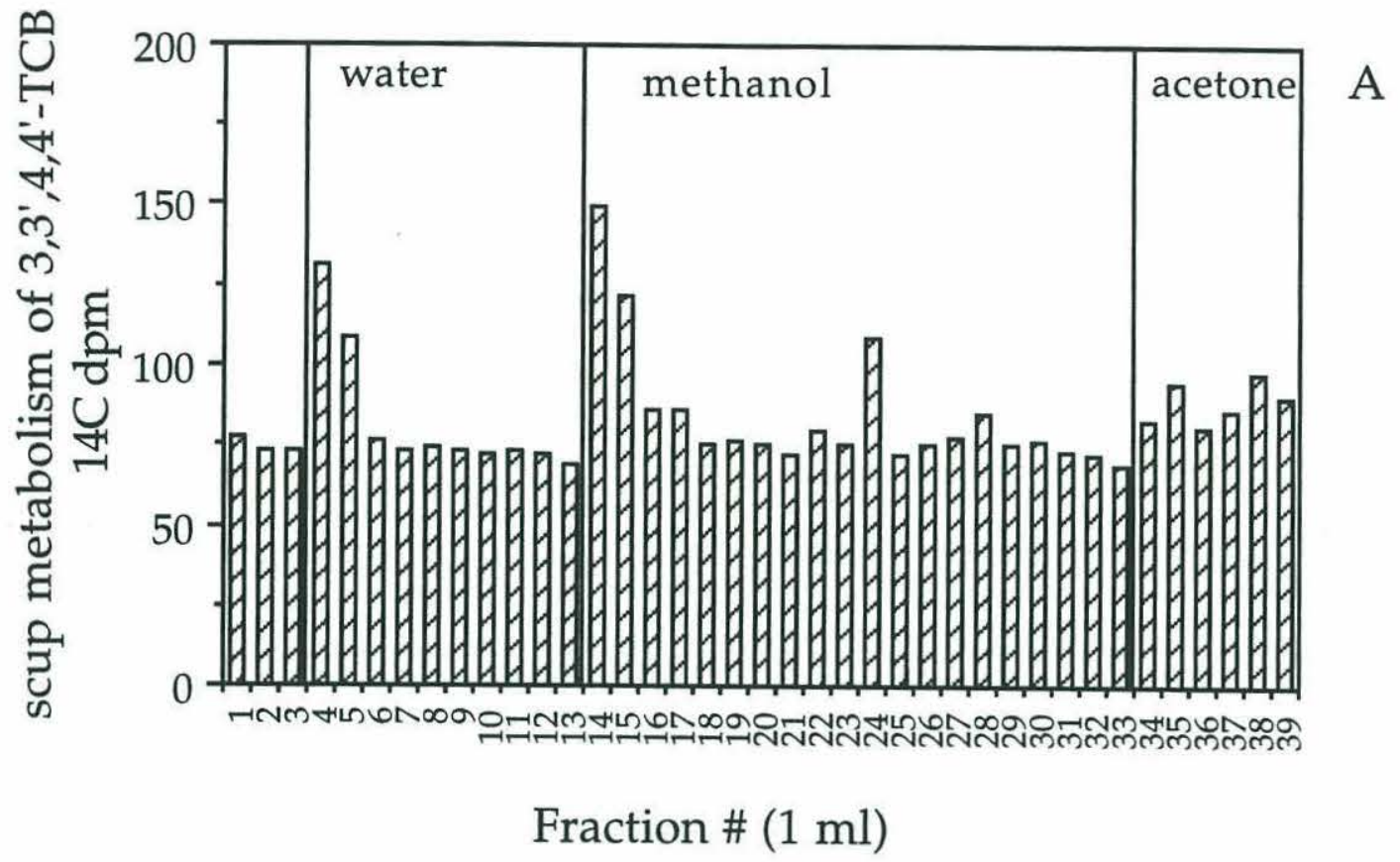

Figure 5. XAD column profiles of 3,3',4,4'-TCB metabolites produced in feral scup hepatic microsomes (A), and in MC-treated rat hepatic microsomes (B), and profile of 3,3',4,4'-TCB (C). Metabolites were generated and separated on the column as described in Methods. Bars represent the radioactivity contained in each $1 \mathrm{ml}$ fraction, eluted from the column as it was washed with $1 \mathrm{ml}$ aliquots of water, methanol, and acetone. The first three (A, B) or first one (C) fractions are $\mathrm{H}_{2} \mathrm{O}$ column blanks; the next fraction was eluted as sample was applied to the column. 


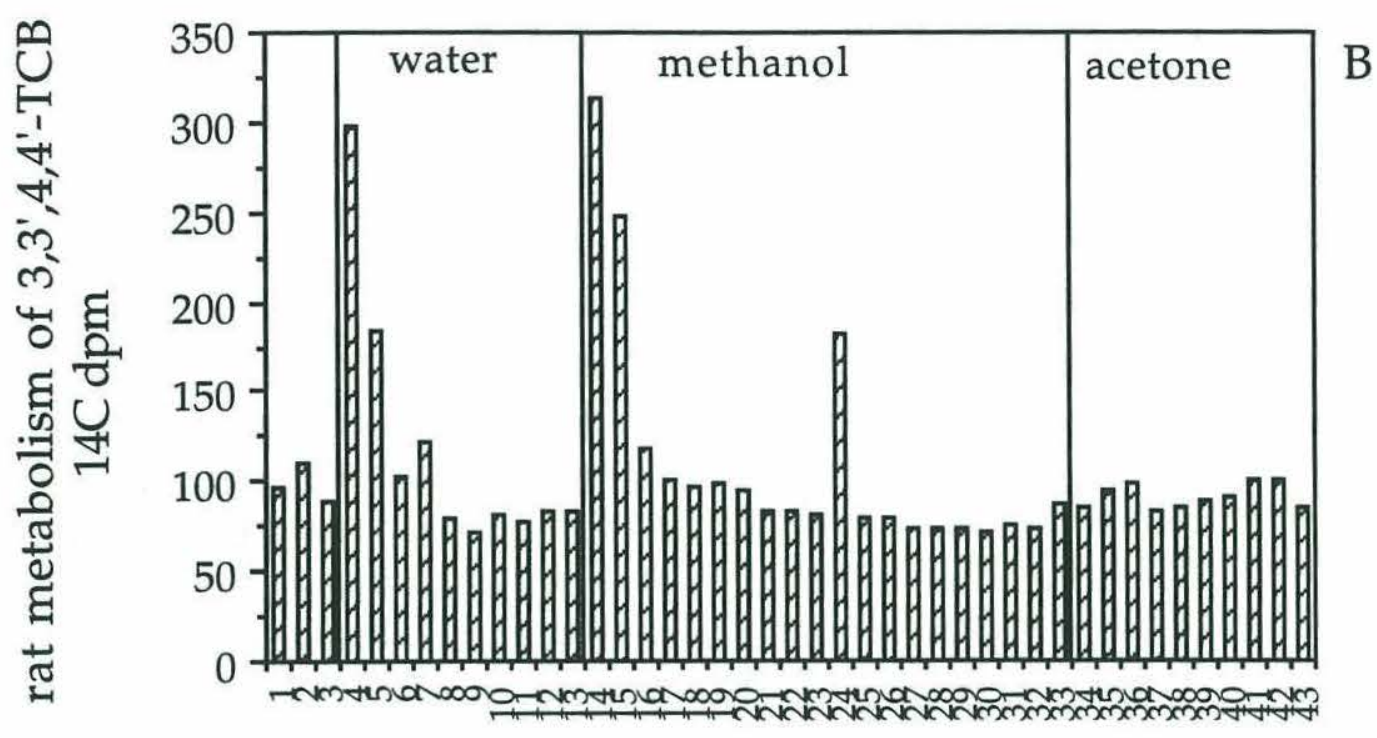

Fraction\# $(1 \mathrm{ml})$

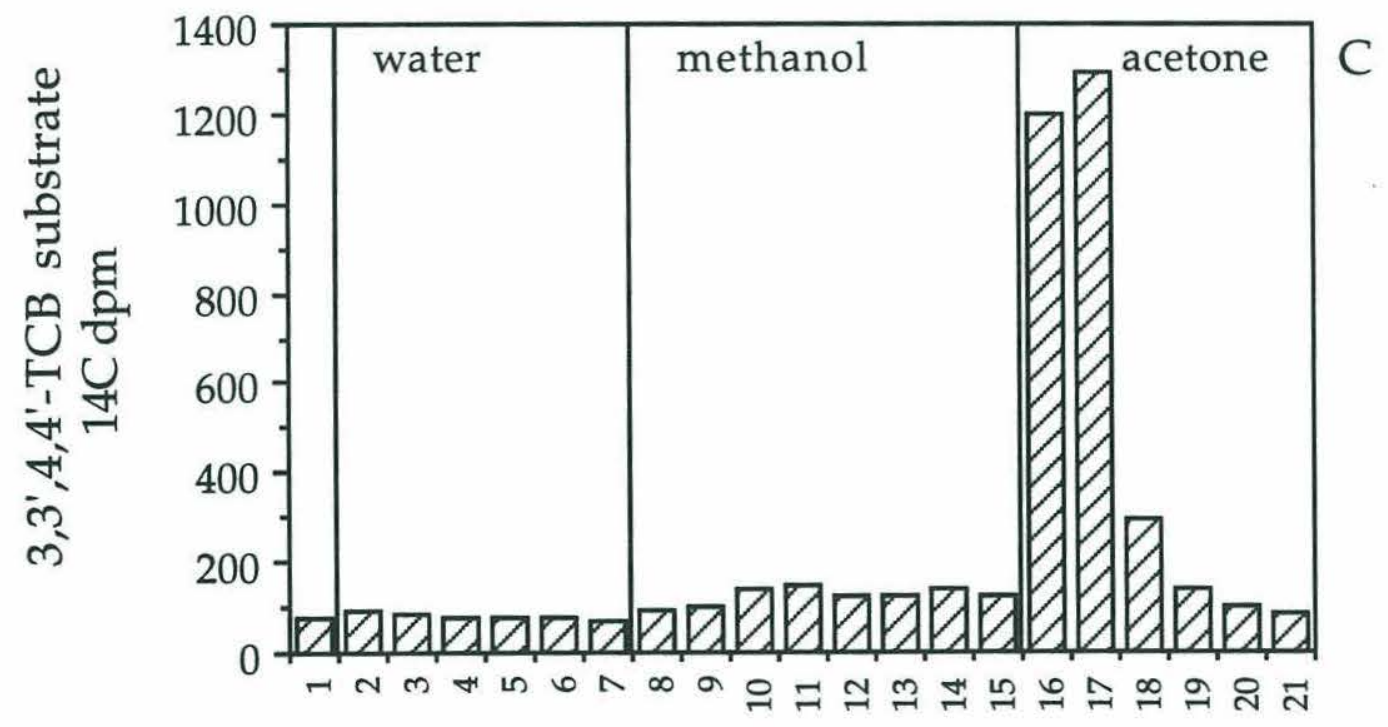

Fraction \# $(1 \mathrm{ml})$ 


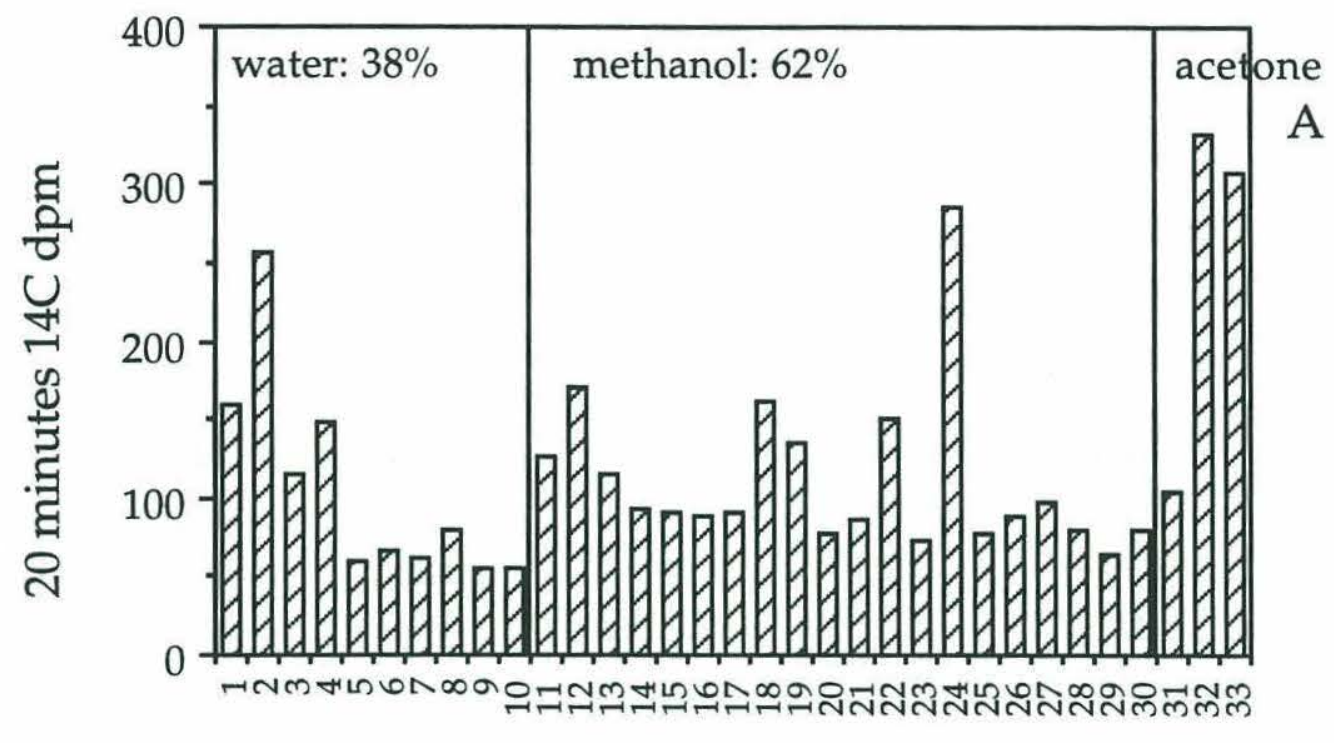

Fraction \# $(1 \mathrm{ml})$

Figure 6. XAD column profiles of 2,2',5,5'-TCB metabolites produced in feral scup hepatic microsomes. Data were obtained as described in Fig 5. Incubations were conducted for 20 minutes (A), 40 minutes (B), or 60 minutes (C). Indicated above the water and methanol fractions is the percentage of total recovered metabolites that eluted in water and methanol, respectively. Water- and methanol-metabolites were quantified by summing the radioactivity in the peak fractions, after subtracting background radioactivity from each fraction. The peaks eluting with acetone are parent TCB (see Results section). 


\section{0 minutes $14 C$ dpm}

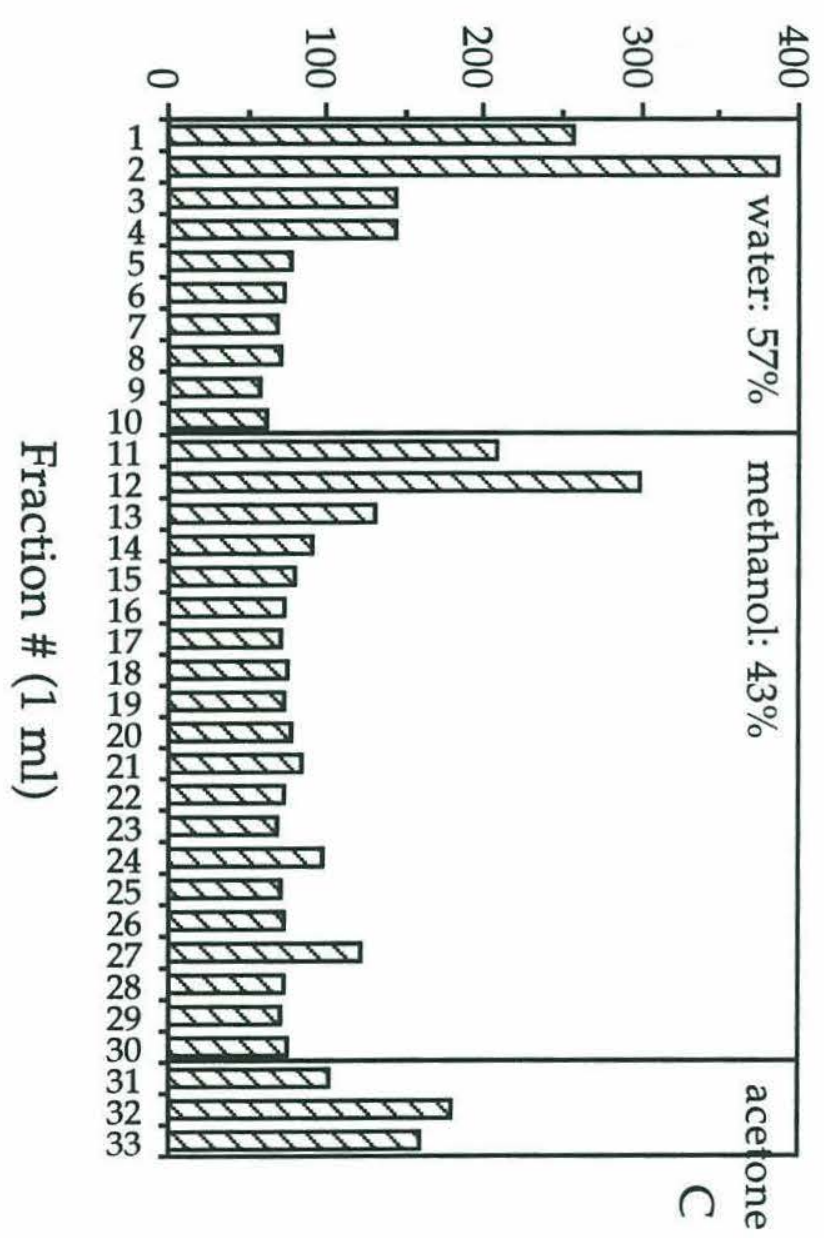

40 minutes $14 \mathrm{C}$ dpm

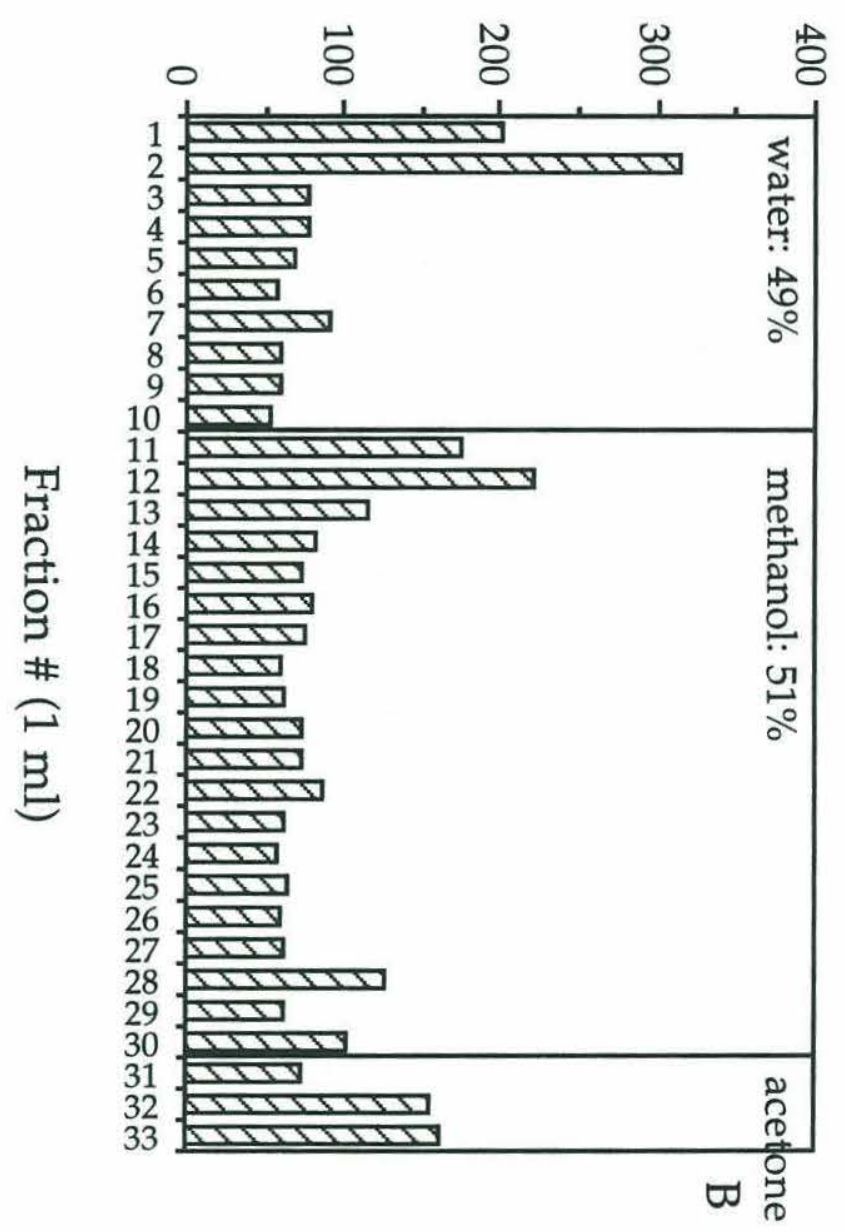


TABLE 1

Microsomal Enzyme Contents and Activities in Depurated, Feral, and Treated Scup

\begin{tabular}{|c|c|c|c|c|c|}
\hline & $\begin{array}{l}\text { control } \\
(n=3)\end{array}$ & $\begin{array}{c}\text { feral } \\
(\mathrm{n}=5 \text { pools })\end{array}$ & $\begin{array}{c}\text { BNF } \\
(n=3)\end{array}$ & $\begin{array}{c}\mathrm{MC} \\
(\mathrm{n}=3)\end{array}$ & $\begin{array}{l}\text { TCDF } \\
(\mathrm{n}=3)\end{array}$ \\
\hline $\begin{array}{l}\text { cytochrome P450 } \\
(\mathrm{nmol} / \mathrm{mg})\end{array}$ & $0.130 \pm 0.024$ & $0.446 \pm 0.185 *$ & $0.179 \pm 0.036$ & $0.449 \pm 0.108 *$ & $0.682 \pm 0.206 * * *$ \\
\hline $\begin{array}{l}\text { EROD } \\
(\mathrm{nmol} / \mathrm{min} / \mathrm{mg})\end{array}$ & $0.105 \pm 0.064$ & $0.715 \pm 0.391$ & $1.410 \pm 0.084$ & $4.054 \pm 2.461 * *$ & $5.011 \pm 1.764 * *$ \\
\hline $\begin{array}{l}\text { CYP1A } \\
\text { (pmol P450E/mg) }\end{array}$ & $0.4 \pm 0.7$ & $67 \pm 40$ & $47 \pm 7$ & $219 \pm 58 * *$ & $288 \pm 119 * * *$ \\
\hline $\begin{array}{l}\text { CYP2B } \\
\text { (pmol P450B/mg) }\end{array}$ & $6 \pm 7 \# \# \#$ & $122 \pm 51 * * *$ & $11 \pm 3 \# \#$ & $30 \pm 13 \# \#$ & $68 \pm 23 * \#$ \\
\hline $\begin{array}{l}3,3^{\prime}, 4,4^{\prime}-\mathrm{TCB}-\mathrm{OH} \\
(\mathrm{pmol} / \mathrm{min} / \mathrm{mg})\end{array}$ & $0.09 \pm 0.10$ & $0.13 \pm 0.07$ & $0.23 \pm 0.11 *$ & $0.21 \pm 0.02$ & $0.23 \pm 0.02 *$ \\
\hline $\begin{array}{l}2,2^{\prime}, 5,5^{\prime}-\mathrm{TCB}-\mathrm{OH} \\
(\mathrm{pmol} / \mathrm{min} / \mathrm{mg})\end{array}$ & $0.2 \pm 0.1 \# \# \#$ & $2.1 \pm 0.4 * * *$ & $1.3 \pm 0.1 * * * \# \#$ & $0.9 \pm 0.2 * * \# \# \#$ & $1.2 \pm 0.3 * * * \# \#$ \\
\hline
\end{tabular}

Assays were conducted as described in Methods. Individual fish were depurated of environmental residues and treated with either corn oil (control) or with the CYP1A inducers BNF, MC, or TCDF. Feral samples were prepared from pooled livers of freshly-caught animals, 20-70 fish per sample. (Three feral scup pools were assessed for rate of metabolism of 3,3',4,4'-TCB, two BNF samples were assessed for CYP2B content, all other sample sizes are as indicated.) Values are means \pm SD ( $n-1)$. Sample groups are significantly greater than control at a level: ${ }^{*} \mathrm{p}<0.05,{ }^{*} \mathrm{p}<0.01,{ }^{* * *} \mathrm{p}<0.001$, or sample groups are significantly less than feral at a level: \# p<0.05, \#\# p<0.01, \#\#\# p<0.001, based on a one-factor ANOVA test. 
was significant only in fish treated with BNF or TCDF. The rate of metabolism of $2,2^{\prime}, 5,5^{\prime}$-TCB by feral fish was significantly elevated over rates in all groups of depurated fish (Table 1). The average rate of metabolism of 3,3',4,4'-TCB in feral scup microsomes $(0.13 \mathrm{pmol} / \mathrm{min} / \mathrm{mg}$ microsomal protein) was one-fifteenth that of 2,2',5,5'-TCB (2.1 $\mathrm{pmol} / \mathrm{min} / \mathrm{mg}$ ), and in control scup the rates differed by two-fold.

A strong positive correlation was seen between rate of metabolism of 2,2',5,5'TCB and immunodetectable content of the putative scup CYP2B $\left(r^{2}=0.670\right.$; Fig 7). A weak positive correlation was seen between rate of metabolism of 3,3',4,4'-TCB and immunodetectable CYP1A1 content in each fish $\left(r^{2}=0.101\right.$; Fig 7). No correlation was seen for 2,2',5,5'-TCB metabolism versus CYP1A1 content, nor for 3,3',4,4'-TCB metabolism versus CYP2B content (Fig 7). We hypothesized that the weakness of the correlation between rate of 3,3',4,4'-TCB metabolism and microsomal CYP1A1 content was related to the fact that the rates of this activity were so slow. The variability in interindividual response to a given chemical treatment (the range or standard deviation) was as great as the response itself (the mean activity rate in each treatment group). Thus, patterns of response were visible only when group means were plotted, rather than individual values. Upon plotting means (Fig 8), correlations became stronger for 2,2',5,5'-TCB metabolism versus CYP2B content $\left(r^{2}=0.690\right)$, and for 3,3',4,4'-TCB metabolism versus CYP1A1 content $\left(\mathrm{r}^{2}=0.451\right)$. Again, no correlations were seen for the reverse plots (Fig 8). These data are consistent with scup CYP1A1 being a principal catalyst for the in vitro metabolism of the coplanar congener 3,3',4,4'-TCB, and the putative CYP2B a principal catalyst for the in vitro metabolism of the di-ortho-substituted congener 2,2',5,5'TCB.

\section{Inhibition of TCB metabolism by TCBs}

To further identify the catalysts responsible for metabolism of the two TCB congeners in vitro, one ${ }^{14} \mathrm{C}$-labeled $\mathrm{TCB}$ congener was used as substrate, and the other congener added as an unlabeled "competitor". The addition of cold 2,2',5,5'-TCB caused a moderate elevation in the rate of metabolism of ${ }^{14} \mathrm{C}$-labeled 3,3',4,4'-TCB (Table 2). The addition of cold 3,3',4,4'-TCB caused a very slight drop in the rate of metabolism of ${ }^{14} \mathrm{C}$-labeled $2,2^{\prime}, 5,5^{\prime}$-TCB. These results indicate that 2,2',5,5'-TCB does not inhibit the metabolism of 3,3',4,4'-TCB, while $3,3^{\prime}, 4,4^{\prime}-\mathrm{TCB}$ may bind with a relatively weak affinity to the catalyst for 2,2',5,5'-TCB metabolism. The data imply the presence of two separate enzymes, one principally responsible for the in vitro metabolism of 3,3',4,4'-TCB and the other principally responsible for the in vitro metabolism of 2,2',5,5'-TCB. 

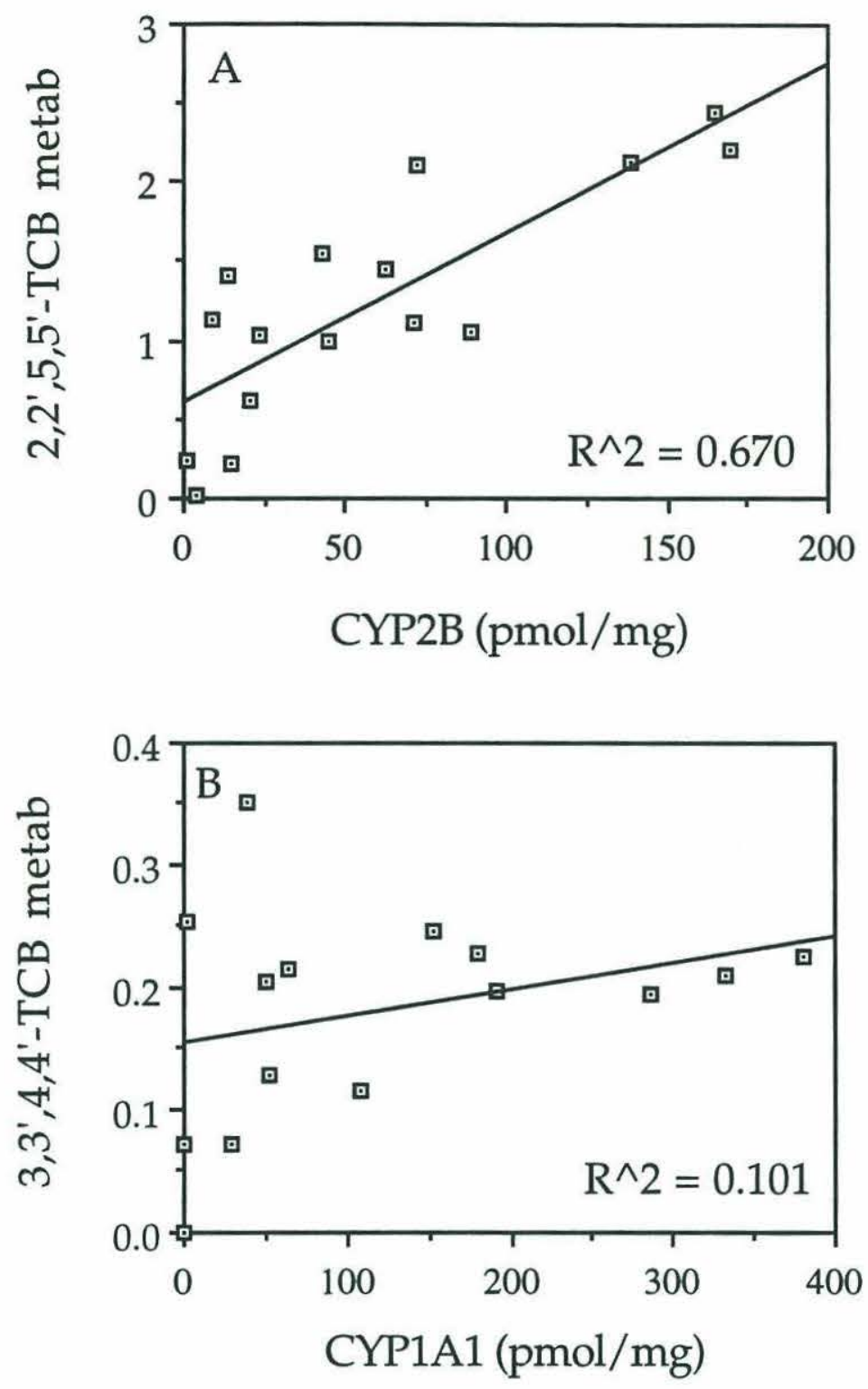

Figure 7. Rate of metabolism of TCB congeners in vitro versus immunodetectable $\mathrm{P} 450$ in scup hepatic microsomes. Data points are for individual livers (control, BNF, MC, TCDF) or for pooled samples (feral), and are the same data used for Table 1. TCB metabolism was assayed radiometrically, and is expressed as $\mathrm{pmol} / \mathrm{min} / \mathrm{mg}$ microsomal protein. Content of cytochromes P450 was determined by Western blotting. (A) 2,2',5,5'-TCB metabolism versus CYP2B content. (B) 3,3',4,4'-TCB metabolism versus CYP1A1 content. (C) 2,2',5,5'-TCB metabolism versus CYP1A1 content. (D) 3,3',4,4'-TCB metabolism versus $\mathrm{CYP} 2 \mathrm{~B}$ content. Three feral pools were assessed for rate of metabolism of 3,3',4,4'-TCB, and five were assessed for rate of metabolism of 2,2',5,5'-TCB. 

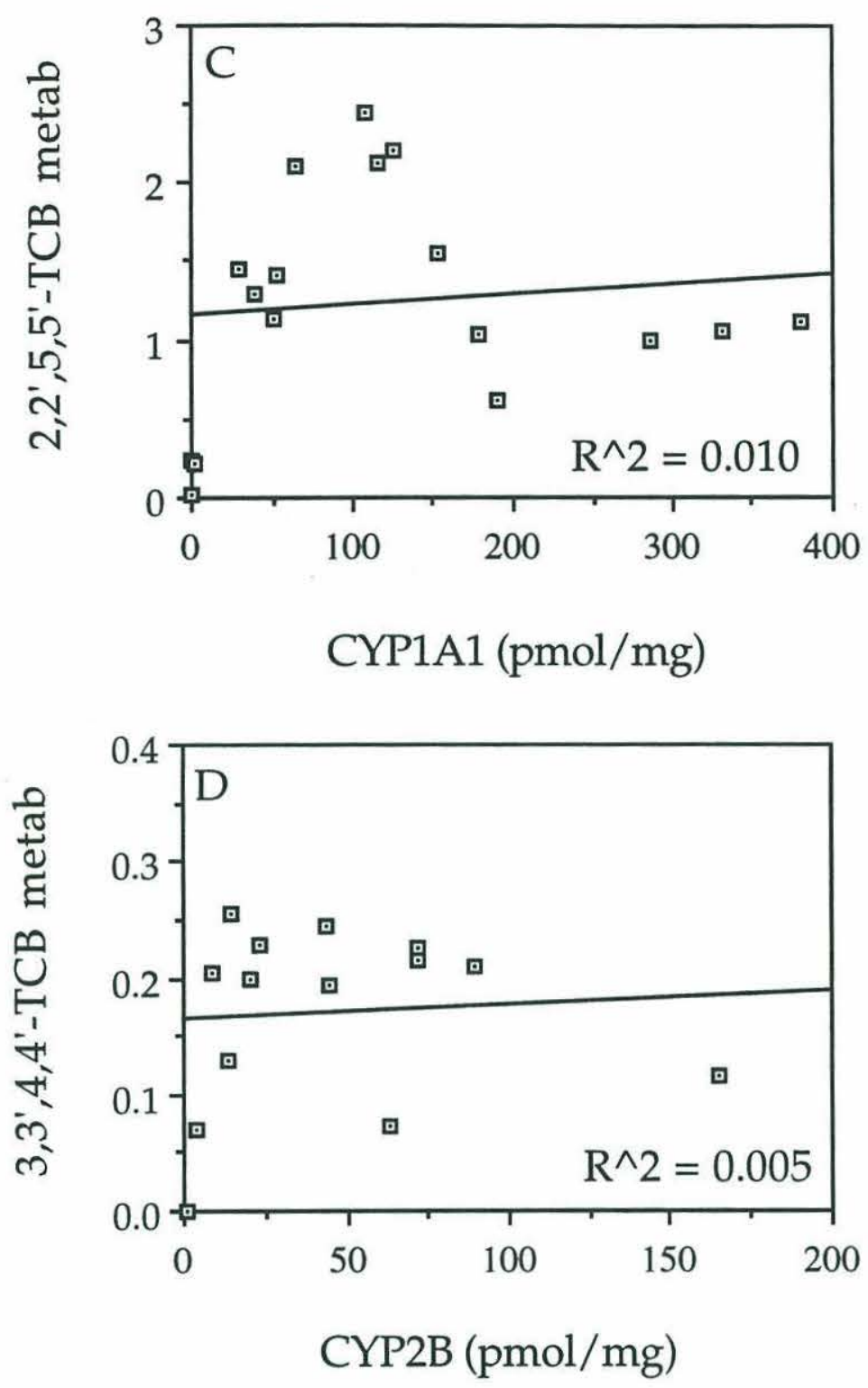

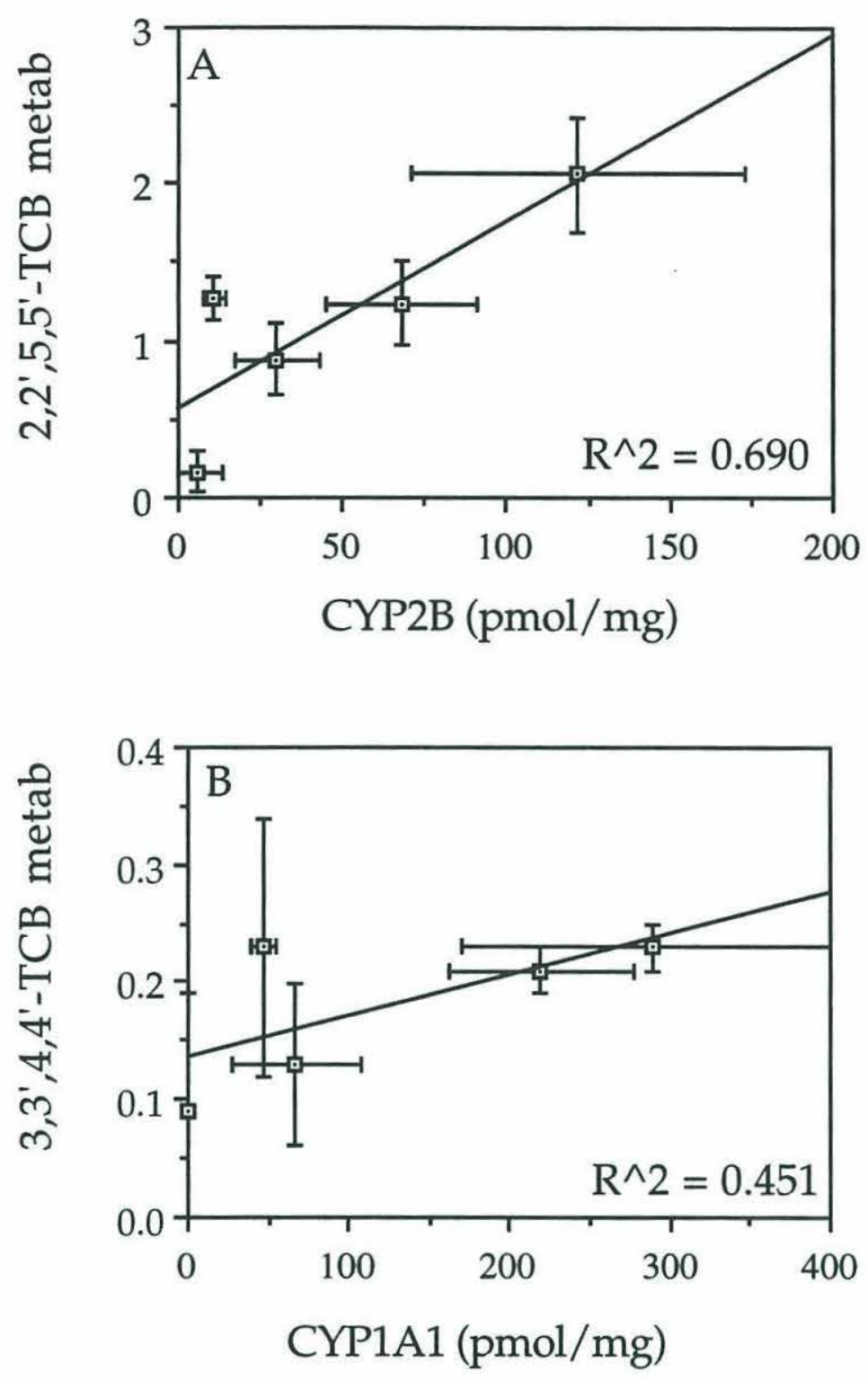

Figure 8. Mean rate of TCB metabolism versus mean content of immunodetectable cytochromes $\mathrm{P} 450$ in hepatic microsomes from control scup, feral scup, and scup treated with BNF, MC, or TCDF. Data points are means and standard deviations of measurements for each treatment group, and are taken from Table 1. All data were obtained as described in Fig 7. (A) 2,2',5,5'-TCB metabolism versus CYP2B content. (B) 3,3',4,4'-TCB metabolism versus CYP1A1 content. (C) 2,2',5,5'-TCB metabolism versus CYP1A1 content. (D) 3,3',4,4'-TCB metabolism versus CYP2B content. 

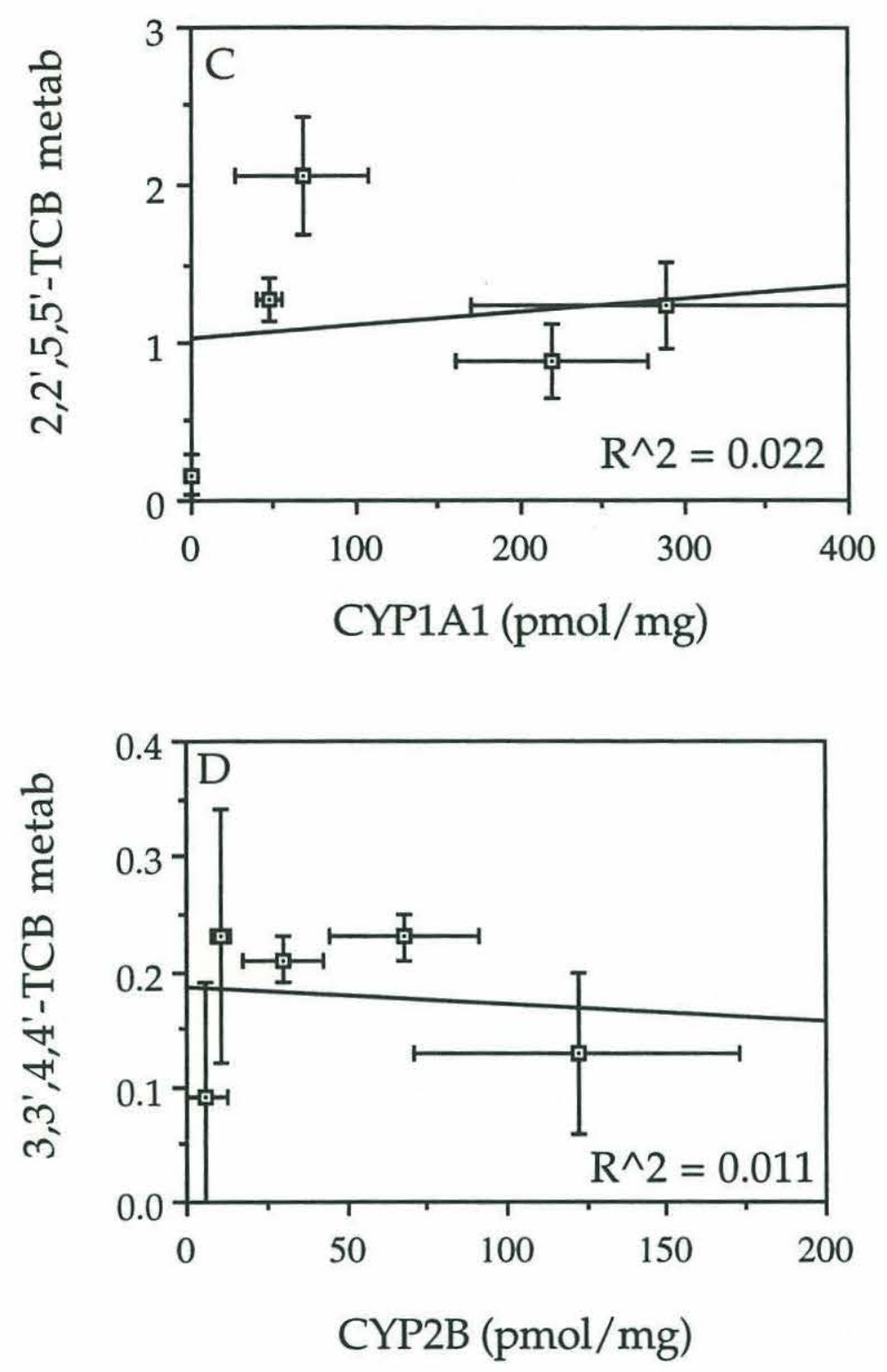
TABLE 2

Rate of Metabolism of ${ }^{14}$ C-TCB Congeners in Hepatic Microsomes from Feral Scup: Addition of a "Competing" TCB Congener

\begin{tabular}{llll}
\hline substrate & competitor & $\mathrm{dpm} a$ & $\mathrm{pmol} / \mathrm{min} / \mathrm{mg} b$ \\
\hline${ }^{14} \mathrm{C}-3,3^{\prime}, 4,4^{\prime}-\mathrm{TCB}$ & control & 367.3 & 0.14 \\
& cold 2,2',5,5'-TCB & 474.0 & 0.24 \\
& blank & 209.0 & \\
\hline${ }^{14} \mathrm{C}-2,2^{\prime}, 5,5^{\prime}-\mathrm{TCB}$ & control & 572.6 & 1.5 \\
& cold 3,3',4,4',-TCB & 482.4 & 1.2 \\
& blank & 132.0 & \\
\hline
\end{tabular}

Microsomes were incubated with ${ }^{14} \mathrm{C}-\mathrm{TCB}$ "substrate" at $2 \mu \mathrm{M}$, and cold TCB "competitor" at $4 \mu \mathrm{M}$. Control assays contained no competitor, blank assays contained no microsomes and were used as a subtractive blank for ${ }^{14} \mathrm{C}$ extraction efficiency. $a$ Disintegrations per minute from ${ }^{14} \mathrm{C}$, measured in extracted aqueous phase of metabolism assay. Each value is a mean of duplicate assays.

$b$ Rates were calculated by subtracting the blanks, then dividing by specific activity of the substrate to convert dpm to pmol. Rates are given in pmol aqueous-soluble metabolites produced/ minute incubation/ mg microsomal protein. 


\section{Inhibition of alkoxyresorufin O-dealkylase activities}

The two TCB congeners were employed at a range of concentrations to assess their ability to inhibit various alkoxyresorufin O-dealkylase activities (Fig 9). $1 \mu \mathrm{M} 3,3^{\prime}, 4,4^{\prime}-\mathrm{TCB}$ inhibited EROD activity by approximately $56 \%$ and MROD activity by approximately $70 \%$. In contrast, $1 \mu \mathrm{M} 2,2$ ',5,5'-TCB inhibited neither of these activities. PROD activity was not inhibited by either congener at any concentration tested (Fig 9C).

\section{Inhibition of TCB metabolism by antibodies}

EROD activity, catalyzed principally by CYP1A1 in scup (Klotz et al., 1983), was used as a control to test for polyclonal antibody specificity. The anti-scup CYP1A1 polyclonal antibody, when added at $60 \mu \mathrm{g} / \mathrm{pmol} \mathrm{P} 450$, inhibited EROD activity by $41 \%$ with respect to non-immune serum controls (Fig 10A). In contrast, $60 \mu \mathrm{g} / \mathrm{pmol}$ of the anti-scup CYP2B polyclonal antibody inhibited EROD activity by only $13 \%$ with respect to nonimmune serum controls. Thus the antibodies showed some degree of specificity for EROD inhibition.

The metabolism of 2,2',5,5'-TCB was inhibited almost completely by $60 \mu \mathrm{g} / \mathrm{pmol}$ of the anti-CYP2B antibody, whereas the anti-CYP1A1 antibody did not inhibit this activity at all, as compared to non-immune serum controls (Fig 10B). The rate of metabolism of $3,3^{\prime}, 4,4^{\prime}-\mathrm{TCB}$ was reduced to near zero by $60 \mu \mathrm{g} / \mathrm{pmol}$ of either anti-CYP2B or nonimmune serum (Fig 10C), suggesting that this activity is susceptible to strong inhibition by nonspecific interactions. The anti-CYP1A1 antibody caused a dramatic increase in the rate of metabolism of 3,3',4,4'-TCB, particularly at low titres (Fig 10C; see Discussion).

\section{Discussion}

We have quantified rates of metabolism of two TCB congeners in hepatic microsomes from the marine fish scup, providing the first direct demonstration of cytochrome P450 involvement in PCB metabolism by teleost fish. The metabolism of $3,3^{\prime}, 4,4^{\prime}-\mathrm{TCB}$ was inhibitable by cytochrome c, ANF, and $\mathrm{CO}$ gas, indicating that it is a P450-catalyzed activity. Aqueous-soluble products were detected following microsomal incubation with either $3,3^{\prime}, 4,4^{\prime}-\mathrm{TCB}$ or $2,2^{\prime}, 5,5^{\prime}-\mathrm{TCB}$, suggesting the capacity for in vivo biotransformation. Microsomal metabolism of 2,2',5,5'-TCB was more rapid than that of $3,3^{\prime}, 4,4^{\prime}-\mathrm{TCB}$, consistent with prior work in mammals suggesting that PCB congeners with adjacent, unsubstituted meta-para positions are more susceptible to enzymatic attack (Matthews and Tuey, 1980; Boon et al., 1989; Borlakoglu and Wilkins, 1993). 


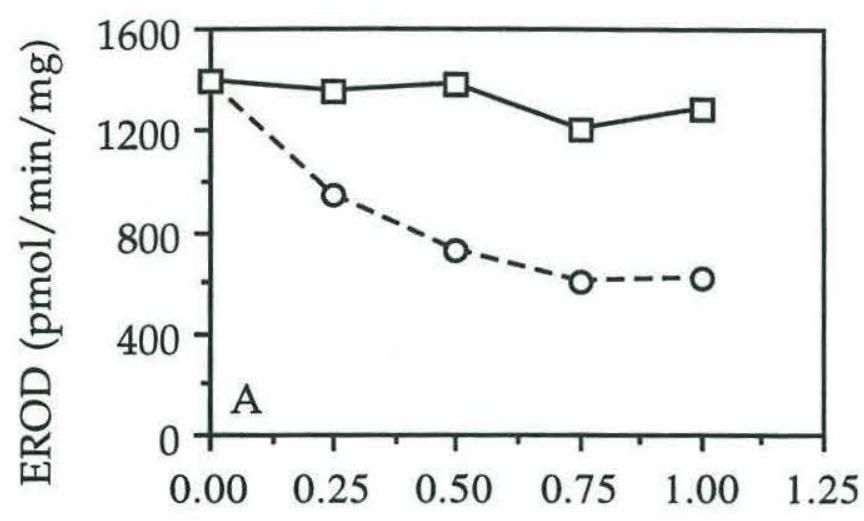

$\begin{array}{cc}--0^{--} & 3344-\mathrm{TCB} \\ -\square- & 2255-\mathrm{TCB}\end{array}$
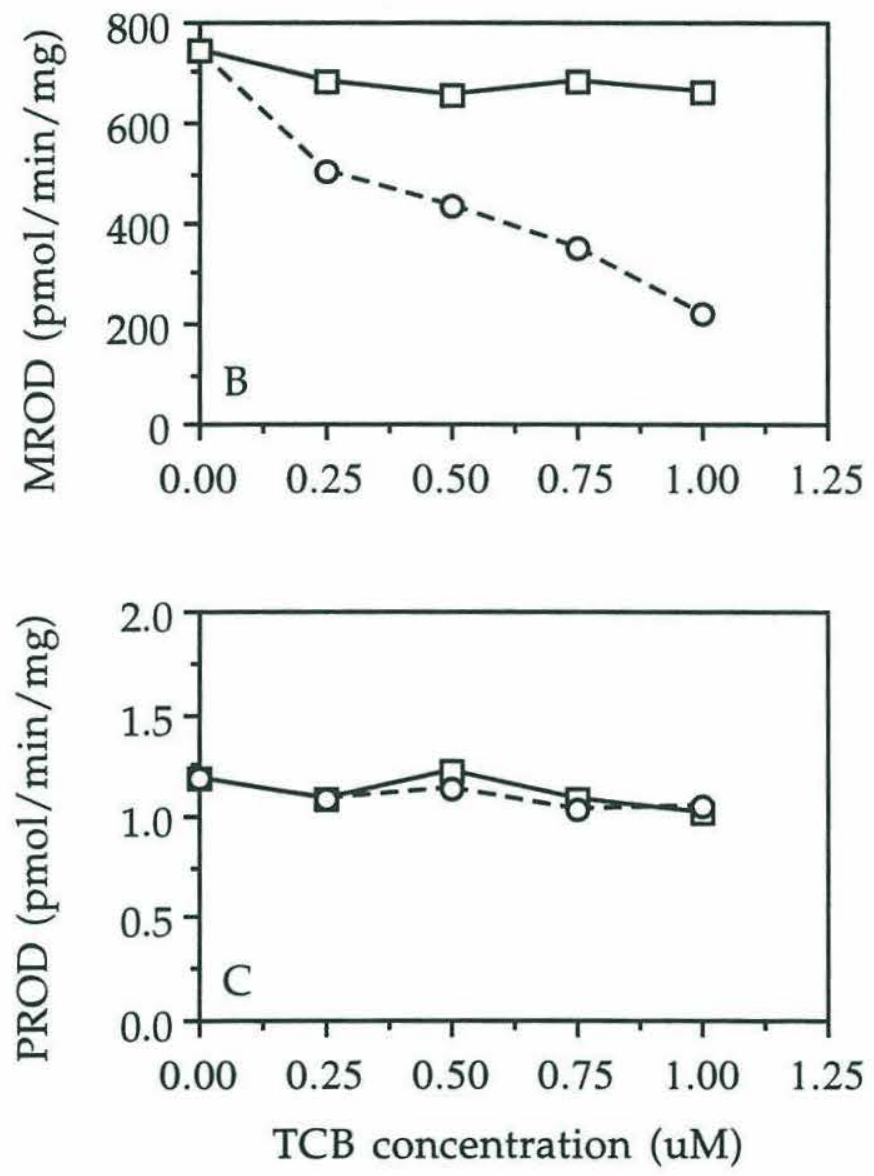

Figure 9. Inhibition of alkoxyresorufin O-dealkylase activities by TCB congeners. Plotted is rate of activity (y) versus TCB concentration in the incubation (x), for (A) EROD, (B) MROD, (C) PROD. Activities were measured in hepatic microsomes from feral scup, using a fluorometric assay as described in Methods. Each TCB congener was added in acetone at concentrations of $0,0.25,0.5,0.75$, and $1.0 \mu \mathrm{M}$. Each point is a mean of duplicate assays, and all results were reproducible in duplicate experiments. 



Figure 10. Polyclonal antibody inhibition of metabolic activities in hepatic microsomes from feral scup. Plotted is rate of activity (y) versus antibody titre in the incubation (x), for (A) EROD activity, (B) 2,2',5,5'-TCB metabolism, and (C) 3,3',4,4'-TCB metabolism. EROD activity was assayed fluorometrically and TCB metabolism radiometrically, as in Methods. Antibodies used were 119p, raised against scup CYP1A1; 7-94, raised against scup CYP2B; and c-93, which is non-immune rabbit serum. Rate of activity in controls containing no serum is indicated by the point at $x=0$. Each point is a mean of duplicate assays, and results were reproducible in duplicate experiments. 
Structure/activity relationships in TCB metabolism

Induction responses, correlation analyses, and inhibition studies all indicate that PCB metabolism in teleost fish follows the same substrate SARs that have been observed in rat. Specifically, it appears that the coplanar congener 3,3',4,4'-TCB is metabolized principally by the scup CYP1A1 homologue, while the di-ortho-substituted congener 2,2',5,5'-TCB is metabolized principally by scup P450B. Immunochemical cross-reactivity with rat CYP2B1 suggests that P450B is likely a CYP2B homologue (Stegeman et al., 1990a; Stegeman et al., 1990b). Elevated rates of metabolism of 3,3',4,4'-TCB were seen in scup that had been treated with CYP1A inducers, with a positive correlation among treatment groups between CYP1A1 content and rate of metabolism of 3,3',4,4'-TCB. Similarly, there was a strong positive correlation among treatment groups between content of the putative CYP2B and rate of metabolism of 2,2',5,5'-TCB.

Although the metabolism of 3,3',4,4'-TCB was strongly inhibited by ANF (Fig 3), the metabolism of 2,2',5,5'-TCB was inhibited to nearly the same extent by each ANF concentration (data not shown). ANF was formerly believed to be a specific inhibitor of CYP1A enzymes, but recent evidence (Chang et al., 1994) indicates that it inhibits other human cytochromes P450 as well, including an enzyme of the CYP2B subfamily. Therefore, we interpret the ANF inhibition of TCB metabolism to suggest a role for cytochrome P450, and not for any specific P450 form.

The anti-scup CYP2B polyclonal antibody strongly inhibited the metabolism of 2,2',5,5'-TCB, while the anti-scup CYP1A1 polyclonal antibody did not, further suggesting a role for CYP2B in this activity. The metabolism of 3,3',4,4'-TCB was not amenable to antibody inhibition studies, due to the very low rates in scup hepatic microsomes. This activity was inhibited almost completely by both anti-scup CYP2B and by non-immune serum, suggesting extreme sensitivity to inhibition by nonspecific interactions. The anti-scup CYP1A1 polyclonal antibody caused a slight elevation in the rate of metabolism of 2,2 ',5,5'-TCB (60\%), and a strong elevation in the rate of metabolism of 3,3',4,4'-TCB (250\%), when added at $15 \mu \mathrm{g} / \mathrm{pmol}$. Such elevations in activity are commonly reported in the use of polyclonal antibodies, and are attributed to nonspecific binding. In the case of this antibody, the modest inhibition of EROD activity (41\%), and the nonspecific stimulation of TCB metabolism, suggest that it is not an effective inhibitory antibody. Nonetheless, the strong stimulation of 3,3',4,4'-TCB metabolism by this antibody provides further suggestion that this may be a CYP1A1catalyzed activity, with the stimulation owing to anti-CYP1A1 / CYP1A1 binding in such a way as to stabilize the catalyst and enhance the rate of 3,3',4,4'-TCB metabolism. 
Inhibition studies using alkoxyresorufin substrates further shed light on the TCB substrate SARs. Studies with rat have shown that EROD activity is catalyzed principally by CYP1A1, MROD activity principally by CYP1A2, and PROD activity largely by CYP2B1/2B2 (Burke et al., 1985). Scup express what appears to be a single CYP1A protein in liver microsomes, which bears sequence similarity to both mammalian CYP1A1 and CYP1A2, but is apparently more closely related to CYP1A1 (Morrison et al., submitted). Scup CYP1A1 is the principal catalyst for EROD activity in hepatic microsomes (Klotz et al., 1983) and is likely also a catalyst for MROD activity. We found that 3,3',4,4'-TCB strongly inhibited both EROD and MROD activities, whereas 2,2',5,5'-TCB did not (Fig 9), suggesting that 3,3',4,4'-TCB binds to the scup CYP1A1 active site and 2,2',5,5'-TCB does not. This is consistent with prior work (Gooch et al., 1989) showing that $3,3^{\prime}, 4,4^{\prime}-$ TCB is a competitive inhibitor of EROD activity in scup hepatic microsomes with $\mathrm{Ki}=0.3 \mu \mathrm{M}$, and that 2,2',5,5'-TCB and 2,2',4,4'-TCB do not inhibit EROD activity. In a more recent study using lower protein concentrations and more sensitive measurement techniques, a $\mathrm{Ki}$ as low as $0.02 \mu \mathrm{M}$ was determined for EROD inhibition by $3,3^{\prime}, 4,4^{\prime}-\mathrm{TCB}$ (Hahn, unpublished observation). This inhibition is consistent with CYP1A1 being a catalyst for the metabolism of 3,3',4,4'-TCB, whereas the lack of such inhibition by 2,2',5,5'-TCB suggests that it is not a CYP1A1 substrate. PROD activity is catalyzed largely by CYP2B forms in rat liver microsomes (Burke et al., 1985), although the principal catalyst for this activity has not been identified in fish. The very low rate of PROD activity catalyzed by scup hepatic microsomes was not inhibited by either TCB congener, possibly suggesting that a P450 form other than CYP1A1 or CYP2B contributes to this activity.

\section{Induction of $3,3^{\prime}, 4,4^{\prime}-\mathrm{TCB}$ metabolism}

Although CYP1A1 content and EROD activity were highly induced by treatment of fish with Ah receptor agonists, the metabolism of 3,3',4,4'-TCB was relatively refractory to induction. Thus, scup treated with MC had 40-fold greater rates of EROD activity than corn-oil treated scup, but only 2 -fold greater rates of TCB metabolism. Similarly, a microsomal sample from an MC-treated rat was found to have a 70-fold higher rate of EROD activity, but only a 6-fold higher rate of TCB metabolism, than did a microsomal sample from a corn-oil treated rat (unpublished observation). 3,3',4,4'-TCB has been found to bind tightly to scup CYP1A1 in hepatic microsomes, evidenced by EROD inhibition (see above Discussion), and co-precipitation using polyclonal antibodies raised against scup CYP1A1 (Joy et al., unpublished observation). The reason for such slow 
metabolism of a substrate which binds tightly and specifically to the scup CYP1A1 active site is unclear.

The slow metabolism in vitro may be due, in part, to the substrate concentration used: metabolism assays were generally carried out at $2 \mu \mathrm{M} 3,3^{\prime}, 4,4^{\prime}-\mathrm{TCB}$, which was determined to be optimal in kinetic studies, and which is commonly used in mammalian metabolism studies. However, the Ki for CYP1A1 inhibition by TCB was estimated to be from $0.3 \mu \mathrm{M}$ down to $0.02 \mu \mathrm{M}$ in scup (see above). Thus, substrate concentration in the assay was 7 to 100 times higher than the concentration that elicits half-maximal inhibition of EROD activity. It is possible that substrate inhibition was partially responsible for the low rates of TCB metabolism in vitro. The assay was not routinely carried out at lower substrate concentrations, however, because assays containing $0.2 \mu \mathrm{M}$ TCB had rates of metabolism that were barely detectable (data not shown).

In a separate study (chapter 2), we present evidence to indicate that TCB elicits catalysis-dependent inactivation of scup CYP1A1 in vitro. The inactivation likely involves partial uncoupling of the $\mathrm{P} 450$ catalytic cycle, and consequent generation of reactive oxygen species, during TCB occupation of the CYP1A1 active site (chapter 2). Thus, the active pool of microsomal CYP1A1 is gradually inactivated during incubation with TCB and NADPH cofactor, which likely contributes to the slow rates of TCB metabolism in vitro. The slow, uncoupled metabolism of TCB may be due to steric hindrance, with the bulky chlorine substituents preventing facile oxygenation of the substrate. This would explain the apparent paradox presented by the tight binding of TCB in the CYP1A1 active site, yet the very slow rate of CYP1A1 metabolism of TCB.

\section{Teleost CYP2B}

The elevated level of the putative scup CYP2B homologue in hepatic microsomes from feral scup is a novel finding. To date there is no conclusive evidence that chemicals can elicit CYP2B induction in fish. Several investigators have exposed fish to mammalian CYP2B inducers, and failed to see elevation in enzyme activities that are catalyzed by mammalian CYP2B enzymes (Addison et al., 1977; Eisele et al., 1984; Addison et al., 1987; Ankley et al., 1987; Goksøyr et al., 1987; Elskus and Stegeman, 1989; Haasch et al., 1994). A few investigators have treated fish with mammalian CYP2B inducers, and have reported increases in CYP2B-like activities (Gutman and Kidron, 1971; Burns, 1976; Forlin and Lidman, 1978; Bach and Snegaroff, 1989; Jensen et al., 1991). However, in the latter studies the reported increases in activity were either very slight (Gutman and Kidron, 1971, p-chloro-N-methylaniline N-demethylase; Jensen et al., 1991, aldrin epoxidase), or were not necessarily CYP2B-like activities (Forlin and Lidman, 1978, $p$ - 
nitroanisole O-demethylase; Bach and Snegaroff, 1989, 7-ethoxycoumarin O-deethylase). In one study (Burns, 1976) there was a reported elevation in aldrin epoxidase activity in treated fish, but this activity actually declined when expressed per nmol cytochrome P450, indicating that there was no selective increase in CYP2B-like activity. Thus, none of these papers provides convincing evidence for actual induction of a CYP2B-like activity, and in no case was there reported an elevation in a fish protein homologous to mammalian CYP2B.

Against this background of results, the much greater content of immunodetectable P450B, the putative scup CYP2B homologue, in feral scup than in depurated scup suggests that some environmental residue or combination of residues may have brought about elevated levels of CYP2B. The feral fish had elevated rates of 2,2',5,5'-TCB metabolism, a CYP2B-like activity, in terms of both specific activity (activity per mg) and estimated turnover number (activity per nmol P450), suggesting elevation (if not actual induction) by exogenous chemicals. Exogenous chemical inducers would likely have been lost during the one-year depuration period, resulting in lowered levels of CYP2B in all groups of depurated/treated scup in our study. The nature of such compounds, and whether they resemble mammalian PB-like inducers, is not known and will require further study. In all groups of fish treated with CYP1A-like inducers, there was a slight elevation in CYP2B content (Table 1), suggesting that similar compounds in the environment could contribute to CYP2B elevation in feral fish. However, the ratio of microsomal CYP2B content to CYP1A1 content was far higher in the feral fish (1.8) than in the fish treated with CYP1A inducers $(0.1-0.2)$, indicating P450 regulation by different factors. Alternatively, the elevated CYP2B content in feral fish may involve as yet undefined physiological or hormonal regulation of this enzyme.

\section{Implications of TCB metabolism}

The rates of TCB metabolism in scup are far lower than rates of metabolism of polycyclic aromatic hydrocarbons in the same species. For example, Stegeman et al. (1981) report a rate of metabolism of benzo[a]pyrene of $250 \mathrm{pmol} / \mathrm{min} / \mathrm{mg}$ microsomal protein in hepatic microsomes from feral scup, and $964 \mathrm{pmol} / \mathrm{min} / \mathrm{mg}$ in hepatic microsomes from MCtreated scup. One might deduce from in vitro data that metabolism is a relatively slow route of removal of PCBs, as compared to other xenobiotics, from the tissues of fish. Yet, we have detected appreciable quantities of hydroxylated metabolites of 3,3',4,4'-TCB in bile and liver of TCB-treated fish, including 2-OH-, 4-OH-, 5-OH-, 6-OH-TCB, and two dihydroxy products (White et al., in preparation). Several investigators have presented PCB residue data suggesting that metabolism may enhance $\mathrm{PCB}$ elimination from fish. Brown 
(Brown, 1992) conducted an analysis of PCB residues in tissues of 32 teleost species, and based on relative depletion of PCB congeners, inferred CYP1A-like PCB metabolism in 18 species and CYP2B-like PCB metabolism in 4 different species. Some investigators have noted selective loss from fish tissues of 3,3',4,4'-TCB and 3,3',4,4',5-PeCB (Niimi and Oliver, 1983; de Boer et al., 1993), which suggests CYP1A-like activity. Other investigators have noted selective loss from fish tissues of 2,2',5,5'-TCB and several other congeners with adjacent, unsubstituted meta-para positions (Boon et al., 1989), which suggests CYP2B-like activity. Thus, even low rates of metabolism could contribute to PCB disposition over time.

PCB metabolism, even at relatively slow rates, could result in the production of toxic or reactive PCB derivatives. Mono-hydroxy metabolites of ortho-chlorinated PCBs, including 2,2',5,5'-TCB, have estrogenic properties in mouse (Korach et al., 1988). A metabolite of 3,3',4,4'-TCB was found to interfere with vitamin A disposition in mouse and rat (Brouwer and van den Berg, 1986). Several studies have indicated the metabolic production of unstable PCB intermediates, which alkylate cellular macromolecules (Hesse et al., 1978; Shimada and Sato, 1978; Hargraves and Allen, 1979; Morales and Matthews, 1979; Shimada and Sato, 1980). The presence of PCB metabolites in tissues may thus render fish susceptible to a range of deleterious effects.

In conclusion, we provide evidence indicating that the marine fish scup can metabolize both $3,3^{\prime}, 4,4^{\prime}$-TCB and 2,2',5,5'-TCB to aqueous-soluble derivatives in vitro, and that such metabolism is accomplished by CYP1A1 and the putative CYP2B, respectively. These results imply that PCB metabolism in teleosts may generally follow the same substrate SARs that have been suggested for rat. Such metabolism may provide a route for PCB detoxification and elimination in vivo, but may lead to the production of toxic or reactive products. Knowledge of the catalysts involved in metabolism is crucial to understanding the factors that may up-regulate or down-regulate the turnover of xenobiotics in exposed organisms. 


\section{CHAPTER SIX}

In vitro Metabolism of Polychlorinated Biphenyl (PCB) Congeners by Beluga Whale (Delphinapterus leucas) and Pilot Whale (Globicephala melaena):

Relationship to Cytochrome P450 Expression 


\section{Abstract}

Marine mammals throughout the world are exposed to PCB contamination, but there is no direct information concerning the mechanisms and capacity for PCB metabolism in these species. We measured rates of metabolism in vitro of two tetrachlorobiphenyl (TCB) congeners, 3,3',4,4'-TCB (IUPAC \#77) and 2,2',5,5'-TCB (IUPAC \#52), in beluga whale and pilot whale, as related to content of selected cytochrome P450 forms. Total cytochromes $\mathrm{P} 450$ and b5 were each present at approximately $0.2 \mathrm{nmol} / \mathrm{mg}$ microsomal protein in livers of both cetacean species. TCB metabolism was assessed by incubating hepatic microsomes with ${ }^{14} \mathrm{C}$-labeled TCB, followed by liquid/liquid extraction and scintillation counting of the aqueous-soluble material. Liver microsomes from male and female beluga metabolized 3,3',4,4'-TCB at rates of approximately 21 and $5 \mathrm{pmol} / \mathrm{min} / \mathrm{mg}$ microsomal protein, respectively, rates that were comparable to those seen in rats treated with CYP1A inducers. Pilot whale hepatic microsomes had very little capacity to transform this congener, with rates ranging from not detectable to $0.3 \mathrm{pmol} / \mathrm{min} / \mathrm{mg}$. These sex and species differences in rates of TCB metabolism were generally consistent with content of immunodetectable CYP1A equivalents. CYP1A content was eleven-fold greater in liver microsomes from male beluga than from female beluga, and was close to the limits of detection in hepatic microsomes of many pilot whales. Inhibition studies with $\alpha$ naphthoflavone and ethoxyresorufin in beluga liver microsomes indicated that CYP1A is likely a major catalyst, but possibly not the sole catalyst, of 3,3',4,4'-TCB metabolism in this species. Microsomal metabolism of 2,2',5,5'-TCB was detectable at similar rates in the two species ( $0.3-1.1 \mathrm{pmol} / \mathrm{min} / \mathrm{mg}$ ), substantially lower than rates reported by some investigators in control rat liver microsomes. Both cetacean species expressed microsomal proteins cross-reactive with polyclonal antibodies raised against rabbit CYP2B4 and dog CYP2B10, but not rat CYP2B1/2. Whether the turnover of 2,2',5,5'-TCB is catalyzed by the cetacean CYP2B-like protein(s) is an open question, as the structure and function of the cross-reactive proteins is still unclear. This study demonstrates that PCBs are metabolized to aqueous-soluble products in cetacean liver, and coupled with residue data from other studies, our data indicate that PCB metabolism plays a role in shaping the patterns of PCB residues found in cetacean tissue. 


\section{Introduction}

Marine mammals are exposed to lipophilic, anthropogenic contaminants throughout the world oceans. Polychlorinated biphenyls (PCB) and other chlorinated organics have been found in the tissues of many cetacean species, including beluga and pilot whales (Tanabe et al., 1987; Muir et al., 1988; Muir et al., 1990; Norstrom et al., 1990; Schantz et al., 1993). These odontocete (toothed whale) species may be particularly susceptible to bioaccumulation and retention of lipophilic organic contaminants, due to their large lipid reservoirs and to their place at the top of oceanic food webs. Despite the high degree of exposure, there is very little direct information concerning the capacity of marine mammals to metabolize chlorinated organics.

PCBs are ubiquitous contaminants in marine ecosystems (Tanabe, 1988; Fowler, 1990) and are characterized by chemical stability and hydrophobicity, which lead to their

persistence in marine sediments and food webs. The coplanar PCB congeners that possess meta and para but no ortho-substituents are Ah-receptor agonists, and elicit a suite of toxic responses in laboratory mammals similar to those elicited by 2,3,7,8-tetrachlorodibenzo- $p$ dioxin (reviewed in Goldstein and Safe, 1989). Some ortho-substituted, non-coplanar PCB congeners have been reported to cause DNA damage (e.g. Stadnicki et al., 1979) and to elicit neurotoxic effects (Shain et al., 1991; Kodavanti et al., 1993) in laboratory mammals. Studies of PCB toxicity in marine mammals are necessarily more limited. Investigations have indicated that dietary PCB is linked to reproductive failure (Reijnders, 1986), vitamin A deficiency, and thyroid hormone deficiency (Brouwer et al., 1989) in common seals.

In addition to toxicity mediated by PCB parent compounds, the process of PCB metabolism may be deleterious as well. PCB congeners and mixtures can be metabolically activated to unstable intermediates which alkylate cellular macromolecules (Hesse et al., 1978; Shimada and Sato, 1978; Hargraves and Allen, 1979; Morales and Matthews, 1979; Shimada and Sato, 1980). Some hydroxylated PCB metabolites have inherent toxicity as well. Mono-hydroxy metabolites of ortho-chlorinated PCBs, including 2,2',5,5'-TCB, have estrogenic properties in the mouse (Korach et al., 1988). A metabolite of 3,3',4,4'TCB was found to interfere with vitamin A disposition in mouse and rat (Brouwer and van den Berg, 1986). In spite of the production of reactive intermediates and metabolites, many studies have found that PCB metabolism is a principal pathway for both detoxification (Yoshimura et al., 1987; Klasson Wehler et al., 1990) and for elimination (reviewed in Birnbaum, 1985) in vertebrates. 
In rodents, the oxidative metabolism of PCBs is catalyzed primarily by cytochromes $\mathrm{P} 450$, which play a role in both the detoxification and the activation of substrates. Studies have indicated that structure-activity relationships govern the metabolism of PCBs by cytochromes $\mathrm{P} 450$, with chlorine position determining which P450 enzyme carries out the transformation. In rat liver, CYP1A forms effectively metabolize congeners with halogenated substituents at one or both para positions, and with adjacent unsubstituted ortho-meta carbons, while PB-inducible CYP2B forms preferentially metabolize congeners with adjacent, unsubstituted meta-para positions (Kaminsky et al., 1981; Kennedy et al., 1981; Shimada and Sawabe, 1983; Mills et al., 1985; Ishida et al., 1991). These studies also indicate that congeners with two or more ortho substituents are more rapidly metabolized by rat CYP2B than by CYP1A, whereas congeners with no ortho substituents are more rapidly metabolized by rat CYP1A than by CYP2B.

The hepatic cytochrome P450 system has been studied in few cetacean species, namely beluga whale (White et al., 1994), minke whale (Goksøyr et al., 1986; Goksøyr et al., 1988), short-finned pilot whale, killer whale, and striped dolphin (Watanabe et al., 1989). There have been no direct studies of PCB metabolism in cetaceans. Several investigators have inferred rates and catalysts involved in cetacean PCB metabolism, based on PCB residues detected in tissues (Tanabe et al., 1988; Duinker et al., 1989; Norstrom et al., 1992). For example, based on the relative enrichment of meta-para unsubstituted PCB congeners in cetacean tissue, it has been suggested that monooxygenase activities characteristic of CYP2B are reduced in cetaceans as compared to terrestrial mammals (Tanabe et al., 1988; Norstrom et al., 1992).

In this study we provide the first direct evidence of chlorobiphenyl metabolism in cetaceans. We describe in vitro metabolism of two tetrachlorobiphenyl (TCB) congeners by hepatic microsomes from beluga whale and pilot whale: 3,3',4,4'-TCB (IUPAC \#77), a rat CYP1A substrate and an Ah receptor agonist, and 2,2',5,5'-TCB (IUPAC \#52), a substrate of PB-inducible rat CYP2B forms. The results demonstrate that these beluga had a relatively substantial capacity to metabolize 3,3',4,4'-TCB, whereas the pilot whales analyzed had a very limited capacity for such metabolism. The relative rates of this activity were consistent with the relative contents of microsomal CYP1A, which were low in most of the pilot whales but appeared to be induced in some of the beluga. Both species metabolized 2,2',5,5'-TCB at low but detectable rates, and immunoblots showed the expression of CYP2B-like proteins in both species. These CYP2B-like proteins may be involved in the metabolism of 2,2',5,5'-TCB in cetaceans, but this remains speculative, as their structure and function are not yet described. 


\section{Materials and Methods}

Chemicals $3,3^{\prime}, 4,4^{\prime}-\mathrm{TCB}$ [U-14 $\mathrm{C}$ ] $(52.1 \mathrm{mCi} / \mathrm{mmol})$ was purchased from Pathfinder (St. Louis, MO). Analysis by GC/ECD and GC/MS revealed no hydrophobic contaminant peaks (> 99\% pure); small polar contaminants were completely removed by dissolving the TCB in hexane and extracting twice with $\mathrm{NaOH} .2,2$ ',5,5'-TCB [U-14 $\mathrm{C}$ ] $(14.2$ $\mathrm{mCi} / \mathrm{mmol}$ ) was purchased from Sigma (St. Louis, $\mathrm{MO}$ ). This product was determined to be $>98 \%$ pure by HPLC analysis, conducted on an ODS- 2 column by the manufacturer. Unlabeled 3,3',4,4'-TCB was obtained from Pathfinder (St. Louis, MO). Purity of the TCB was $>99 \%$ by GC/ECD and GC/MS analysis. Unlabeled 2,2',5,5'-TCB was obtained from Ultrascientific (North Kingstown, RI). Manufacturer analysis by GC/FID indicated $>99 \%$ purity. Ethoxyresorufin was from Molecular Probes (Eugene, OR). All other chemicals used were of the highest grade available from commercial suppliers. Antibodies used are described below.

Tissues Beluga whales (Delphinapterus leucas) from the Husky Lakes of the Tuktoyuktuk peninsula, on the MacKenzie River delta (Canadian Arctic), were hunted by local Inuit in a subsistence harvest in November 1989. Collection and preparation of these samples have been described elsewhere (White et al., 1994). The six beluga samples used in TCB metabolism studies were selected to represent the full range of cytochrome P450 content in this population. These included three females with relatively low (female-typical) P450 and CYP1A, and three males with relatively high (male-typical) P450 and CYP1A.

Pilot whales (Globicephala melaena) were found stranded on Cape Cod, Massachusetts, during December 1990 and September-October 1991. Some animals died naturally, while others were euthanized with pentobarbital by authorized personnel of the New England Aquarium. Liver samples were removed at times ranging from 5 minutes to several hours after death, and immediately frozen in liquid nitrogen. Samples from nine pilot whales were selected for TCB metabolism studies. These included the 6 animals with the shortest time interval between euthanasia and sample freezing (5 to 45 minutes), and thus with the lowest chance of having enzyme degradation in the samples. These were all from the 1991 stranding. Also assayed were 3 animals with relatively high CYP1A content, all from 1990. The latter samples were collected approximately 2 to 3 hours subsequent to death or euthanasia. Hepatic microsomes were prepared by differential centrifugation as previously described (White et al., 1994), and microsomal protein content was measured with the bicinchoninic acid method (Smith et al., 1985), using bovine serum albumin as a standard. 
Hepatic microsomes from rat were provided by Dr. Mark Melancon (U.S. Fish and Wildlife Service, Laurel, MD). Fisher-344 rats were treated by intraperitoneal injection with either corn oil (control, day 1), 3-methylcholanthrene in corn oil (MC, $20 \mathrm{mg} / \mathrm{kg}$, day 1), or phenobarbital in saline (PB, $80 \mathrm{mg} / \mathrm{kg}$, days 1, 2, 3). Animals were killed on day four by decapitation, and microsomes were prepared by differential centrifugation (Melancon et al., 1990).

Microsome characterization The content of microsomal P450 was determined using dithionite difference spectra of CO-treated samples (Stegeman and Binder, 1979), with an extinction coefficient of $91 \mathrm{mM}^{-1} \mathrm{~cm}^{-1}$ (Omura and Sato, 1964). Ethoxyresorufin Odeethylase (EROD) activity in beluga was quantified at $37^{\circ} \mathrm{C}$ using the spectrophotometric kinetic assay of Klotz et al. (1984). EROD activity in pilot whale samples was measured similarly, but rates in many samples were below the limits of detection of the spectrophotometric assay. EROD rates in pilot whale were thus quantified at $32^{\circ} \mathrm{C}$ with a fluorometric kinetic assay, using a Cytofluor 2300 fluorescence plate reader (Millipore), as described previously (Hahn et al., 1993). The fluorometric rates are reported here.

Immunoblot (Western blot) analysis was performed as previously described (Kloepper-Sams et al., 1987; Stegeman et al., 1991). Primary antibodies used were as follows: monoclonal antibody 1-12-3, raised against cytochrome $\mathrm{P} 450 \mathrm{E}$ from the marine fish scup (a CYP1A1 homologue) (Park et al., 1986); polyclonal antibody 7-94, raised against scup cytochrome P450B (Gray, 1988), which is likely a CYP2B form (Stegeman et al., 1990a; Stegeman et al., 1990b); anti-P450PB4, a polyclonal antibody against rat CYP2B1, provided by Dr. David Waxman (Dana-Farber Cancer Institute, Boston, MA) (Frey et al., 1985); anti-P450LM2, a goat polyclonal antibody against raised against PBinducible rabbit CYP2B4, obtained from Oxford Biomedical Research, Inc. (Oxford, MI); and anti-PBD2, a polyclonal antibody raised against PB-inducible dog CYP2B10, provided by Dr. James Halpert (University of Arizona) (Duignan et al., 1987). Antibodies raised against scup $\mathrm{P} 450 \mathrm{~B}$ and rat CYP2B1 show reciprocal cross-reactivity, each recognizing the same protein bands in both rat microsomes and in scup microsomes (White et al., 1994). MAb 1-12-3 has been shown to strongly cross-react with mammalian CYP1A1 but not CYP1A2 (Park et al., 1986; Kloepper-Sams et al., 1987). Secondary antibodies used were alkaline phosphatase linked goat-anti-mouse IgG, goat-anti-rabbit IgG, and rabbit-anti-goat IgG. Blots of pilot whale samples with MAb 1-12-3 were probed and developed using the Enhanced Chemiluminescent Detection kit of Schleicher and Schuell (Keene, NH), according to manufacturer's instructions. 
Content of the P450E homologue (P4501A) in beluga and pilot whale microsomes was quantified by densitometric scanning of MAb 1-12-3-reactive bands with a video imaging system (Masterscan, Scanalytics, division of CSPI, Billerica, MA), and comparison to P450E standards. Because the strength with which MAb 1-12-3 recognizes P450E homologues may differ between species, CYP1A levels deduced from immunoblots represent relative rather than absolute values for each species.

TCB metabolism assay The assay of Schnellman et al. (1983) was used, with modifications, to measure metabolism of 3,3',4,4'-TCB and 2,2',5,5'-TCB. Incubations were performed at $37^{\circ} \mathrm{C}$ under air in a shaking incubator. Incubations were $0.5 \mathrm{ml}$ (unless specified otherwise) and contained $1 \mathrm{mg} / \mathrm{ml}$ microsomal protein, $50 \mathrm{mM}$ Tris, $\mathrm{pH}$ 7.6, 0.1 mM EDTA, and $5 \mu \mathrm{M}{ }^{14} \mathrm{C}$-labeled TCB dissolved in acetone (acetone concentration in the incubations was approximately $3 \%$ ). The TCB concentration used in the assay was similar to concentrations that have been used in studies of TCB metabolism in rat hepatic microsomes (e.g. Ghiasuddin et al., 1976; Mills et al., 1985). After a 2-minute preincubation, reactions were initiated by the addition of $1 \mathrm{mM} \mathrm{NADPH}$ and run for 30 minutes. Reactions were terminated by the addition of $0.75 \mathrm{ml}$ of $0.5 \mathrm{~N} \mathrm{NaOH}$, and heated at $70^{\circ} \mathrm{C}$ for 10 minutes to aid in microsome solubilization (Schnellman et al., 1983). To remove parent TCB, the samples were extracted two to three times with $2.5-\mathrm{ml}$ aliquots of n-hexane : $100 \%$ ethanol (19:1). $500 \mu \mathrm{l}$ of the aqueous/ $\mathrm{NaOH}$ phase was then neutralized with $\mathrm{HCl}$ and combined with $6 \mathrm{ml}$ Scintiverse II (Fisher Scientific), and ${ }^{14} \mathrm{C}$ in this phase was measured with liquid scintillation counting on a Beckman LS5000TD counter. Rate of production of aqueous-soluble metabolites was calculated by subtracting the aqueous radioactivity in blank incubations containing either boiled microsomes or no microsomes (both blanks gave similar results), and converting to pmol of PCB equivalents. The limits of resolution of the assay were approximately $20 \mathrm{dpm}$, giving approximate detection limits of $0.03 \mathrm{pmol} / \mathrm{min} / \mathrm{mg}$ for 3,3',4,4'-TCB metabolism, and $0.1 \mathrm{pmol} / \mathrm{min} / \mathrm{mg}$ for $2,2^{\prime}, 5,5^{\prime}-$ TCB metabolism. Duplicate assays were performed on each sample. The amount of substrate converted to aqueous metabolites was $12 \%$ for male beluga with 3,3',4,4'-TCB, $3 \%$ for female beluga with $3,3^{\prime}, 4,4^{\prime}-\mathrm{TCB}$, and $<1 \%$ for all other assays. It is thus reasonable to assume that substrate depletion did not occur, and that the assay conditions used (30 minutes, $1 \mathrm{mg} / \mathrm{ml}$ ) gave results that were within the linear range for these activities.

Studies were conducted to assure that any residual pentobarbital in pilot whale microsomes was not inhibitory to metabolism of 2,2',5,5'-TCB. Microsomes were preincubated at $37^{\circ} \mathrm{C}$ for 10 minutes in a Tris buffer (see above) with NADPH cofactor, to 
allow for pentobarbital metabolism. ${ }^{14} \mathrm{C}$-labeled TCB was then added to initiate the TCB metabolism assay, which was conducted as described above. The rates of TCB metabolism in these incubations were nearly identical to the rates of duplicate tubes that were not pre-incubated.

Inhibition studies TCB metabolism assays were conducted with beluga hepatic microsomes, as above, in the presence of the CYP1A inhibitor $\alpha$-naphthoflavone (ANF). Microsomes and buffer were combined, ANF added first, then TCB substrate, and reactions were initiated with NADPH. ANF was dissolved in methanol and added at final concentrations of $1 \mu \mathrm{M}, 10 \mu \mathrm{M}$, and $100 \mu \mathrm{M}$ ANF ( $5 \mu$ l added to $250 \mu$ l reaction volume). Methanol alone $(5 \mu \mathrm{l})$ was added to control and blank incubations. Each assay was performed in duplicate.

Fluorometric, kinetic EROD assays were conducted with beluga hepatic microsomes, as described above, in the presence of potential inhibitors. Microsomes, buffer, and ER substrate were combined, ANF or TCB inhibitor added, and reactions were initiated with NADPH. ANF was dissolved in methanol and added at final concentrations of $1 \mu \mathrm{M}, 10 \mu \mathrm{M}$, and $100 \mu \mathrm{M}$ ( $4 \mu \mathrm{l}$ added to $200 \mu \mathrm{l}$ reaction volume); $4 \mu \mathrm{l}$ methanol alone was added to control incubations. The two TCB congeners were each dissolved in acetone and added at final concentrations of $1.0 \mu \mathrm{M}, 0.75 \mu \mathrm{M}, 0.5 \mu \mathrm{M}$, and $0.25 \mu \mathrm{M}(1 \mu \mathrm{l}$ added to $200 \mu \mathrm{l}$ reaction volume); $1 \mu \mathrm{l}$ acetone alone was added to control incubations. Each assay was performed in duplicate. Protein concentration in the EROD assay was 0.2 $\mathrm{mg} / \mathrm{ml}$, and substrate concentration was $2 \mu \mathrm{M}$ ER.

XAD column chromatography Aqueous-soluble metabolites of ${ }^{14} \mathrm{C}$-labeled 3,3',4,4'-TCB were generated in beluga hepatic microsomes as above. Incubations were extracted with hexane/ethanol as above, and the remaining aqueous/ $\mathrm{NaOH}$ sample was applied to an $\mathrm{XAD}$ column. Columns were prepared by pouring $5 \mathrm{~cm}$ of dry XAD-2 (coarse) resin (Amberlite polystyrene beads, Sigma) into a glass pasteur pipette, with a glass wool plug at the bottom. The column was pre-washed with several milliliters each of water, methanol, acetone, and water again, before sample application. Sample was applied and the column was sequentially washed with several milliliters each of water, methanol, and acetone. Each $1 \mathrm{ml}$ fraction was collected and dried in a $60^{\circ} \mathrm{C}$ oven to reduce solvent volume. $5 \mathrm{ml}$ of Scintiverse II was added to each sample, and metabolites were quantified by liquid scintillation counting as above. When parent TCB alone was mixed with assay buffer and added to a column, all radioactivity eluted with acetone washes. 
Statistics Significant differences between sample groups were evaluated with a one-factor ANOVA test, using Statview SE+ Graphics, v.1.03 (Abacus Concepts) for Macintosh.

\section{Results}

\section{Microsomal monooxygenase systems}

The content of microsomal cytochromes $\mathrm{P} 450$ and b5 determined for 18 pilot whales are listed in Table 1, and compared to our previously published results for beluga whale. Values for pilot whales are separated into two groups, which were stranded in separate years. The beluga were presumably all of a single social group. Average values for P450 and b5 ranged from $0.2-0.3 \mathrm{nmol} / \mathrm{mg}$ microsomal protein, in all groups. Three male and three female beluga selected for TCB metabolism studies had average P450 content of 0.325 and $0.182 \mathrm{nmol} / \mathrm{mg}$, respectively (Table 2 ). This sexual dimorphism in enzyme content was typical for the beluga population (White et al., 1994). Nine pilot whale samples were selected for TCB metabolism studies, with average P450 contents of 0.314 and $0.211 \mathrm{nmol} / \mathrm{mg}$ in the 1990 and 1991 subgroups, respectively (Table 2).

Monooxygenase components and activities were not sexually differentiated in the pilot whale populations examined, so mixed-sex averages are presented.

Immunoblot analysis with the CYP1A1-specific monoclonal antibody 1-12-3 revealed a single cross-reactive protein in each pilot whale liver sample (Fig 1). Beluga liver microsomes were previously shown to have a single 1-12-3 cross-reacting protein (White et al., 1994). Until sequence data is available, we will refer to these immunoreactive proteins as members of the P4501A subfamily, or CYP1A homologues. Average specific content of the cetacean CYP1A homologue was similar in beluga and 1990 pilot whale, whereas average content in 1991 pilot whale was significantly less than that in beluga (Table 1). EROD activity, catalyzed predominantly by CYP1A in mammals, varied widely within each species: 24 - $750 \mathrm{pmol} / \mathrm{min} / \mathrm{mg}$ in beluga, $3-520 \mathrm{pmol} / \mathrm{min} / \mathrm{mg}$ in pilot whale. As with CYP1A, average EROD rates for 1990 pilot whale and for beluga were similar, whereas rates in the 1991 pilot whale group were significantly less than those in beluga (Table 1).

Within each species, there was a strong positive correlation between EROD activity and immunodetectable CYP1A content (pilot: Fig 2; beluga: White et al., 1994), consistent with the cetacean CYP1A homologue being a principal catalyst for microsomal EROD activity. Among the animals selected for TCB metabolism studies, the male beluga had 11fold greater CYP1A content and 15-fold higher EROD rates than did the selected females. The 1990 pilot whale sub-group selected for analysis had 30-fold greater CYP1A content 


\section{TABLE 1}

Microsomal Monooxygenase Components and Activities in Pilot and Beluga Liver Samples

\begin{tabular}{lccc}
\hline & $\begin{array}{c}\text { Pilot whale, 1990 } \\
(\mathrm{n}=4)\end{array}$ & $\begin{array}{c}\text { Pilot whale, 1991 } \\
(\mathrm{n}=14)\end{array}$ & $\begin{array}{c}\text { Beluga whale } a \\
(\mathrm{n}=13)\end{array}$ \\
\hline $\begin{array}{l}\text { Cytochrome P450 } \\
\text { (nmol/mg) }\end{array}$ & $0.275 \pm 0.142$ & $0.207 \pm 0.079$ & $0.274 \pm 0.089$ \\
$\begin{array}{l}\text { Cytochrome b5 } \\
\text { (nmol/mg) }\end{array}$ & $0.210 \pm 0.045$ & $0.219 \pm 0.062$ & $0.222 \pm 0.034$ \\
$\begin{array}{l}\text { EROD activity } \\
(\mathrm{pmol} / \mathrm{min} / \mathrm{mg})\end{array}$ & $239 \pm 211$ & $33 \pm 62 *$ & $291 \pm 261$ \\
$\begin{array}{l}\text { CYP1A } \\
(\mathrm{pmol} \mathrm{P450E/mg)} b\end{array}$ & $17 \pm 9$ & $3 \pm 7 *$ & $37 \pm 28$ \\
\hline
\end{tabular}

All values are expressed as sample means \pm standard deviations based on ( $n-1)$.

* Values in 1991 pilot whale are significantly less than those in beluga at $\mathrm{p} \leq 0.01$, based on a one-factor ANOVA test.

$a$ Data reported by White et al., 1994.

$b$ The strength of cross-reaction with MAb 1-12-3 may be species-specific, thus immunochemical comparisons across-species are not strictly quantitative. 


\section{TABLE 2}

TCB Metabolism Rates and P450 Parameters in Selected Cetacean Samples

\begin{tabular}{lllll}
\hline & $\begin{array}{l}\text { Beluga whale, male } a \\
(\mathrm{n}=3)\end{array}$ & $\begin{array}{l}\text { Beluga whale, female } a \\
(\mathrm{n}=3)\end{array}$ & $\begin{array}{l}\text { Pilot whale, 1990 } b \\
(\mathrm{n}=3)\end{array}$ & $\begin{array}{l}\text { Pilot whale, 1991 } b \\
(\mathrm{n}=6)\end{array}$ \\
\hline Cyt P450 (nmol/mg) & $0.325 \pm 0.046$ & $0.182 \pm 0.070$ & $0.314 \pm 0.146$ & $0.211 \pm 0.031$ \\
EROD (pmol/min/mg) & $563 \pm 55 *$ & $38 \pm 22$ & $306 \pm 199$ & $7 \pm 5$ \\
CYP1A (pmol/mg) $c$ & $67.0 \pm 6.7 *$ & $5.9 \pm 2.1$ & $19.4 \pm 8.4$ & $0.6 \pm 0.7$ \\
\hline $3,3^{\prime}, 4,4^{\prime}-\mathrm{TCB}-\mathrm{OH}$ & & & & $0.04 \pm 0.07$ \\
$(\mathrm{pmol} / \mathrm{min} / \mathrm{mg})$ & $21.48 \pm 1.59 \#$ & $4.88 \pm 0.36$ & $0.92 \pm 0.45$ & $0.18 \pm 0.35$ \\
$(\mathrm{pmol} / \mathrm{min} / \mathrm{mmol} \mathrm{P450)}$ & $66.64 \pm 7.39 \#$ & $29.06 \pm 8.68$ & $14.7 \pm 7.5$ & $50.8 \pm 73.3$ \\
$(\mathrm{pmol} / \mathrm{min} / \mathrm{nmol} \mathrm{CYP1A})$ & $321.9 \pm 25.5$ & $870.8 \pm 212.1$ & & $0.40 \pm 0.07$ \\
\hline 2,2',5,5'-TCB-OH & & & & $1.96 \pm 0.49$ \\
$(\mathrm{pmol} / \mathrm{min} / \mathrm{mg})$ & $1.14 \pm 0.10 \ddagger$ & $0.32 \pm 0.10$ & & \\
$(\mathrm{pmol} / \mathrm{min} / \mathrm{mmol} \mathrm{P450)}$ & $3.54 \pm 0.51 \ddagger$ & $1.80 \pm 0.18$ & & \\
\hline
\end{tabular}

All values are expressed as mean \pm standard deviation based on (n-1). Sample groups are statistically different from each other at $\mathrm{p} \leq$ 0.01, as follows: $*$ male beluga $>1990$ pilot $>$ female beluga $=1991$ pilot; $\#$ male beluga $>$ female beluga $>1990$ pilot $=$ 1991 pilot; $\ddagger$ male beluga $>$ female beluga $=$ pilot, based on a one-factor ANOVA test.

a Cytochrome P450, CYP1A, and EROD data for the beluga are taken from White et al., 1994.

$b$ Data for pilot whales are mixed-sex averages, as no sex differences were seen.

${ }^{c}$ CYP1A is expressed as pmol of P450E equivalents/mg. 


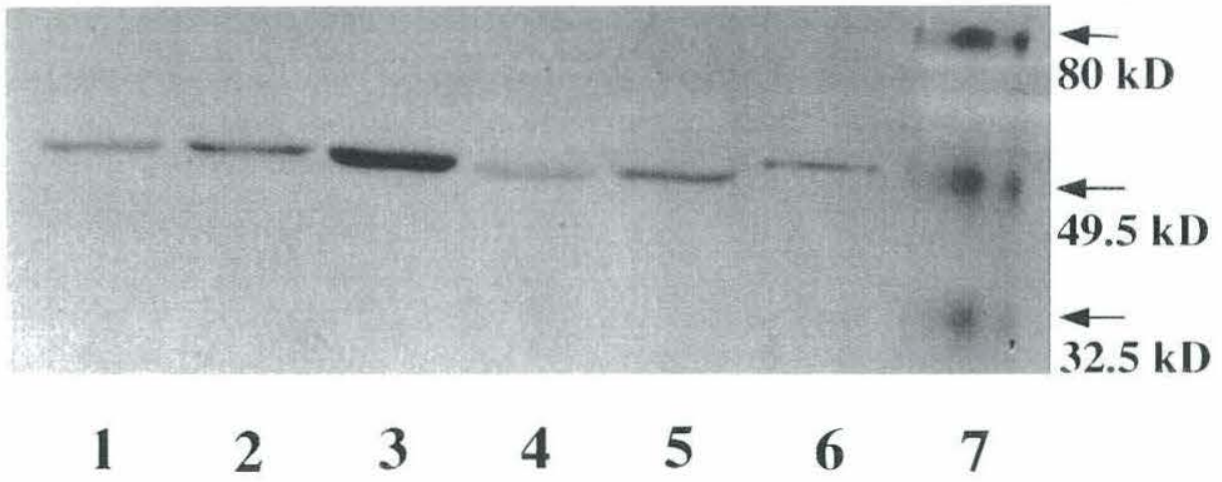

Figure 1. Immunoblot of hepatic microsomes from pilot whale with MAb 1-12-3, raised against scup P450E (CYP1A1). Proteins were separated by SDS-PAGE and transferred to nitrocellulose by electroblotting. The membrane was incubated in $1^{\circ}$ and $2^{\circ}$ antibodies as in Methods, and CYP1A was visualized by alkaline-phosphatase color development. Lanes are as follows: 1 and 2, 1990 samples; 3, 4, and 5, 1991 samples; 6, scup hepatic microsomes; 7 , molecular weight standards. 


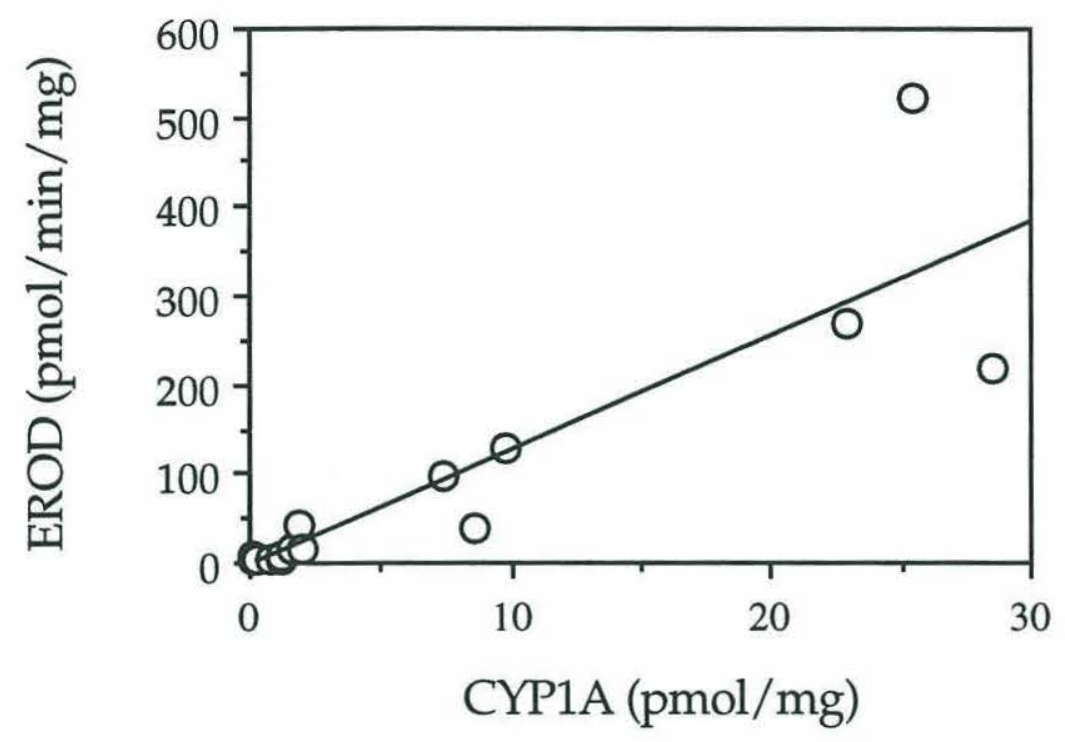

Figure 2. Correlation between hepatic microsomal EROD activity, assayed

fluorometrically, and immunodetectable CYP1A content in 17 pilot whales. Content of the CYP1A homologue was measured as described in Figure 1 and Methods.

$\mathrm{R}^{2}=0.787$. 
and 40-fold higher EROD rates than did the 1991 sub-group; neither CYP1A content nor EROD activity was sexually differentiated in the pilot whales (Table 2).

\section{Immunodetection of cytochrome P4502B}

Hepatic microsomes from both species were immunoblotted with polyclonal antibodies specific for cytochromes P4502B. Neither anti-rat CYP2B1 nor anti-scup P450B crossreacted with cetacean microsomes. However, anti-rabbit CYP2B4 recognized a protein band in the molecular weight region of cytochromes P450 in pilot whale (Fig 3A) and beluga whale (White et al., 1994). Anti-dog CYP2B10 also recognized protein(s) in the $\mathrm{P} 450$ region, in hepatic microsomes from both cetacean species (Figs $3 \mathrm{~B}$ and $3 \mathrm{C}$ ). The major band(s) detected in the cetacean samples by both the rabbit and the dog antibodies appear to be protein(s) of slightly lower electrophoretic molecular weight than those of the immunogens, and slightly lower than that of the major protein detected in liver microsomes from phenobarbital-treated rat (Fig 3). The content of the detected protein(s) did not appear to vary markedly between species or between sexes. We tentatively conclude that the cross-reactive proteins represent members of the CYP2 family in cetacean liver.

\section{TCB metabolism}

The rate of metabolism of 3,3',4,4'-TCB was five times greater in liver microsomes from male beluga than from female, when expressed per milligram of microsomal protein (Table 2). This sex difference remained significant when activity was expressed per nmol total cytochrome $\mathrm{P} 450$, however, females had a greater capacity to metabolize this congener per nmol CYP1A (Table 2). These metabolic rates are comparable to those that have been reported in liver microsomes from rats treated with CYP1A inducers (see Discussion). In contrast, hepatic microsomes from pilot whales of both sexes had very limited capacity to metabolize 3,3',4,4'-TCB (Table 2). Rates in the samples from 1991 were near the limits of detection of the assay, and rates in the samples from 1990 were only slightly greater. In both species, rates of 3,3',4,4'-TCB metabolism were positively correlated with relative content of immunodetectable CYP1A and with rates of EROD activity (Fig 4), consistent with cetacean CYP1A being a principal catalyst for the metabolism of 3,3',4,4'-TCB in vitro.

Rates of metabolism of 2,2',5,5'-TCB were similar in the two whale species (Table 2 ), and were slow by comparison to rates that have been reported in rat liver microsomes (see Discussion). The pilot whales metabolized this congener at rates that were two to ten times faster than the rate of metabolism of 3,3',4,4'-TCB. The belugas metabolized this congener at less than one-tenth the rate of metabolism of 3,3',4,4'-TCB. Rates of 



Figure 3. SDS-PAGE immunoblots of cetacean liver microsomes with anti-CYP2B polyclonal antibodies. (A) Pilot whale microsomes with anti-P450LM2, raised against rabbit CYP2B4. (B) Pilot whale microsomes with anti-PBD2, raised against dog CYP2B10. (C) Beluga whale microsomes with anti-PBD2, raised against dog CYP2B10. Lane 1, PB-treated rat liver microsomes; lanes $2-7$, pilot or beluga whale (70 $\mu \mathrm{g}$ protein/ lane); lane 8, purified rabbit P450LM2 (A) or purified dog CYP2B10 (B,C). 

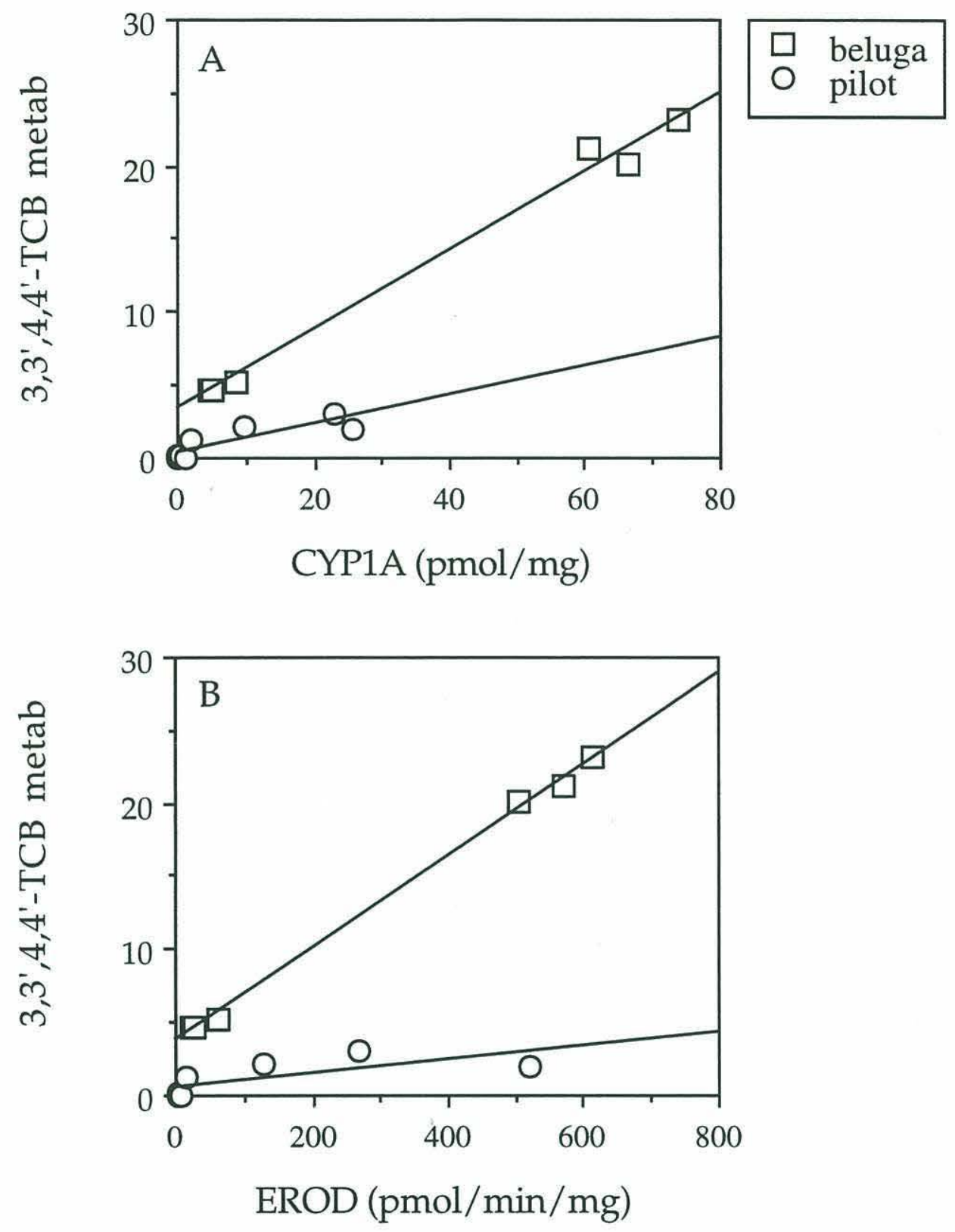

Figure 4. Rate of metabolism of 3,3',4,4'-TCB versus immunodetectable CYP1A content (A) and versus EROD activity (B) in liver microsomes from beluga whale and pilot whale. Squares are beluga, circles are pilot whale. TCB metabolism was assayed radiometrically as described in Methods, and is expressed as pmol/min/mg (beluga) or $10 \mathrm{x} \mathrm{pmol} / \mathrm{min} / \mathrm{mg}$ (pilot). CYP1A content was determined immunochemically as in Figure 1, and EROD activity was assayed either spectrophotometrically or fluorometrically, as outlined in Methods. (A) $\mathrm{r}^{2}=0.990$, beluga, and $\mathrm{r}^{2}=0.756$, pilot. (B) $\mathrm{r}^{2}=0.998$, beluga, and $\mathrm{r}^{2}=$ 0.526 , pilot. 
2,2',5,5'-TCB metabolism in male beluga were slightly, but significantly, greater than those in female (Table 2).

\section{Inhibition studies}

To further define the role of CYP1A in cetacean TCB metabolism, we examined the capacity of the two TCB congeners to inhibit EROD activity in beluga liver microsomes. EROD is catalyzed principally by CYP1A in mammals, including beluga whale (White et al., 1994). The Ki for inhibition of EROD activity in rat liver microsomes has been found to be $0.015 \mu \mathrm{M}$ for 3,3',4,4'-TCB and $0.54 \mu \mathrm{M}$ for 2,2',5,5'-TCB (De Matteis et al., 1989). In beluga, however, the TCB congeners were far less inhibitory toward EROD activity. Concentrations of $0.25-1.0 \mu \mathrm{M} 3,3^{\prime}, 4,4^{\prime}-\mathrm{TCB}$ inhibited EROD activity by $10 \%$ - 16\%, while similar concentrations of 2,2',5,5'-TCB did not inhibit EROD activity at all (Fig 5). In contrast, ANF, which is known to be a strong inhibitor of mammalian CYP1A in vitro (Testa and Jenner, 1981), inhibited beluga EROD activity by $97 \%$ at $1.0 \mu \mathrm{M}$ (Fig $5)$.

Concentrations of ANF up to $100 \mu \mathrm{M}$ had limited capacity to inhibit the metabolism of either TCB congener in beluga hepatic microsomes (Fig 6). Metabolism of 3,3',4,4'TCB was inhibited by $36-47 \%$, and metabolism of $2,2,5,5^{\prime}-\mathrm{TCB}$ by $0-15 \%$. By contrast, $100 \mu \mathrm{M}$ ANF inhibited beluga EROD activity completely (Fig 6), and inhibited benzo[a]pyrene metabolism by $60-100 \%$ in beluga liver microsomes (White et al., 1994).

\section{Metabolite characterization}

Metabolites of 3,3',4,4'-TCB generated in vitro by beluga liver microsomes were separated with liquid chromatography on $\mathrm{XAD}$ resin (Fig 7A). Incubations carried out with several beluga samples were extracted once with hexane:ethanol (19:1) to remove most of the parent compound, and then pooled for metabolite analysis. Following sample application, peaks of radioactivity eluted from the column with the first $2 \mathrm{ml}$ of water, the first $4 \mathrm{ml}$ of methanol, and the first $4 \mathrm{ml}$ of acetone. The acetone peak did not appear when metabolism assays were extracted once more with hexane:ethanol prior to application on the column (not shown). When parent TCB alone was added to the column, it adhered to the matrix during water and methanol washes and only eluted with acetone (Fig 7B). We therefore attribute the acetone peak in figure 7A to unmetabolized parent TCB. The peaks that elute with water and methanol contain specifically-labeled compounds of a more polar nature than TCB itself, which we conclude to be hydroxylated TCB derivatives generated during in vitro metabolism of ${ }^{14} \mathrm{C}$-labeled $\mathrm{TCB}$. The presence of two discrete metabolite peaks 


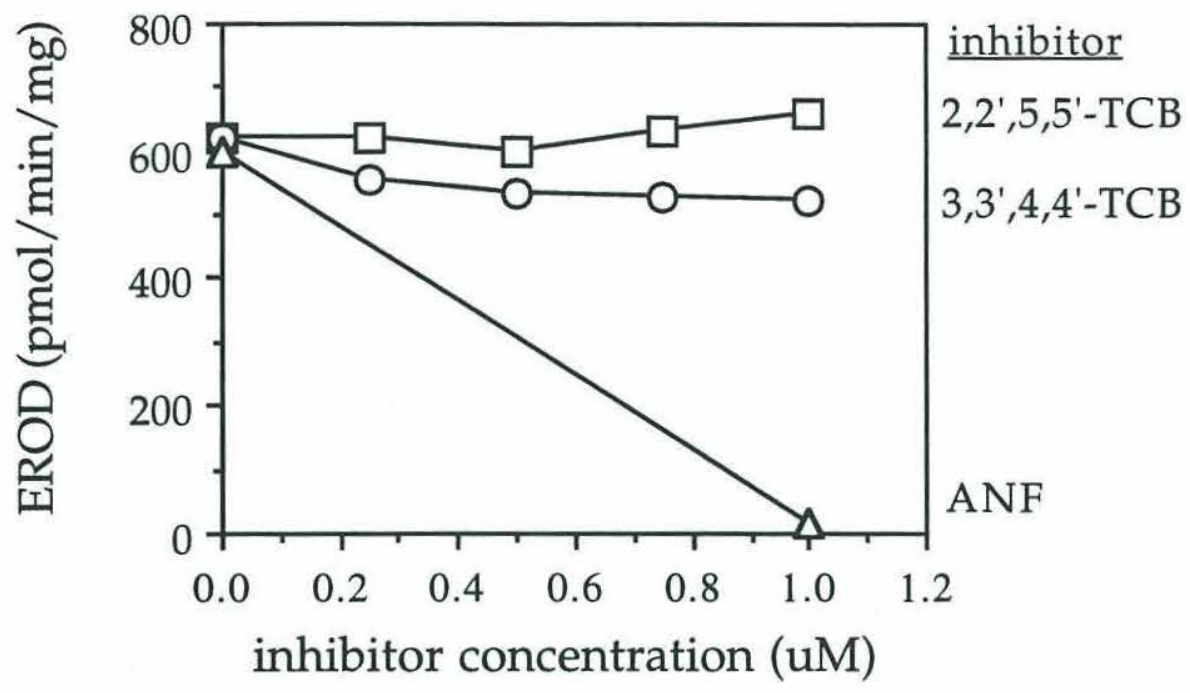

Figure 5. Inhibition of EROD activity in beluga liver microsomes by TCB and ANF. EROD activity was assayed fluorometrically, using a pooled sample of two male belugas. Potentially inhibitory compounds were added at concentrations of $0.25-1.0 \mu \mathrm{M}, \mathrm{ER}$ substrate was used at $2 \mu \mathrm{M}$, and protein concentration was $0.2 \mathrm{mg} / \mathrm{ml}$, as detailed in Methods. Each point is a mean of two measurements. 


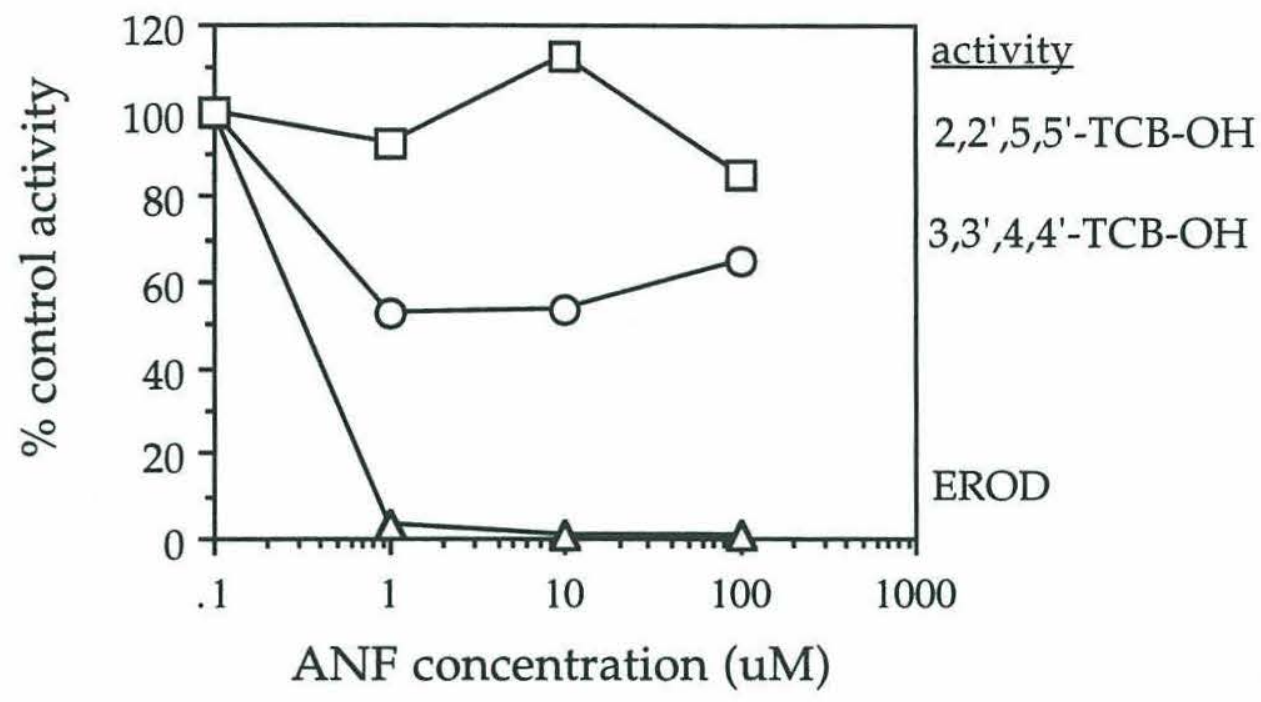

Figure 6. Inhibition of enzyme activities in beluga liver microsomes by ANF. TCB metabolism assays were conducted radiometrically, using a pooled sample of two male belugas ( $1 \mathrm{mg}$ microsomal protein/ $\mathrm{ml}$ assay). EROD activity was measured fluorometrically on the same sample (at $0.2 \mathrm{mg} / \mathrm{ml}$ ) as described in Methods. ANF was dissolved in methanol and added to assays at final concentrations of $1 \mu \mathrm{M}, 10 \mu \mathrm{M}$, and 100 $\mu \mathrm{M}$. Each point is a mean of two measurements. 


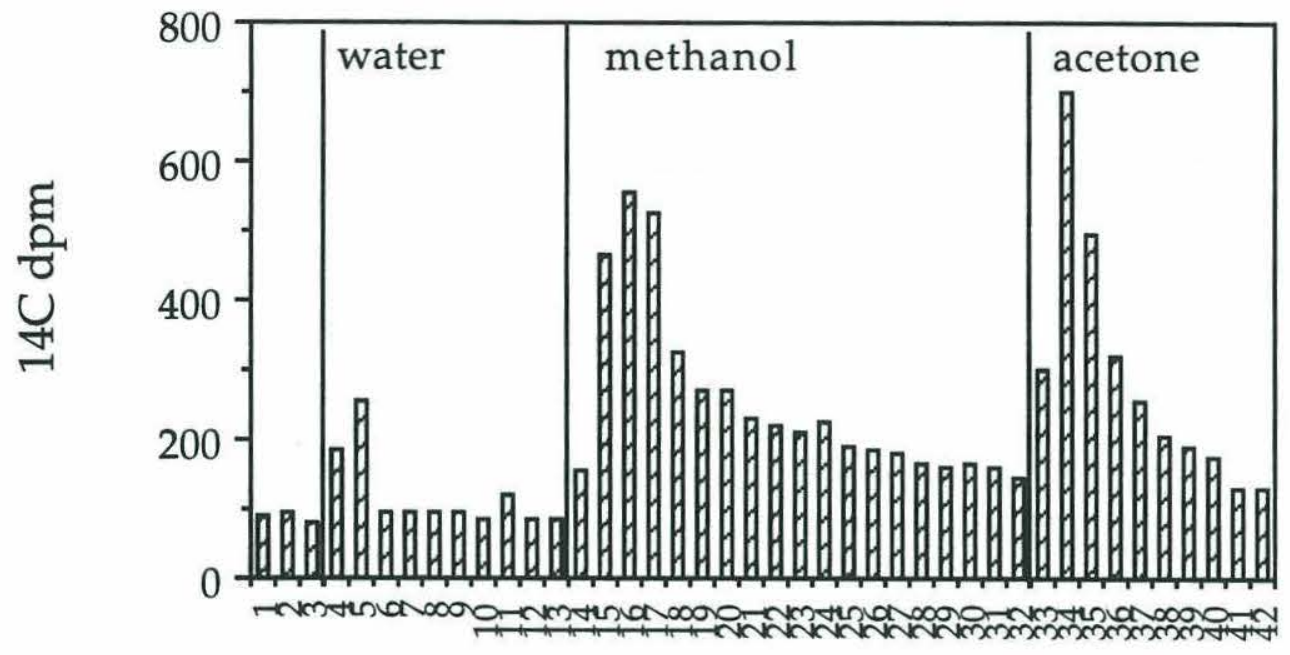

A

Fraction \# $(1 \mathrm{ml})$

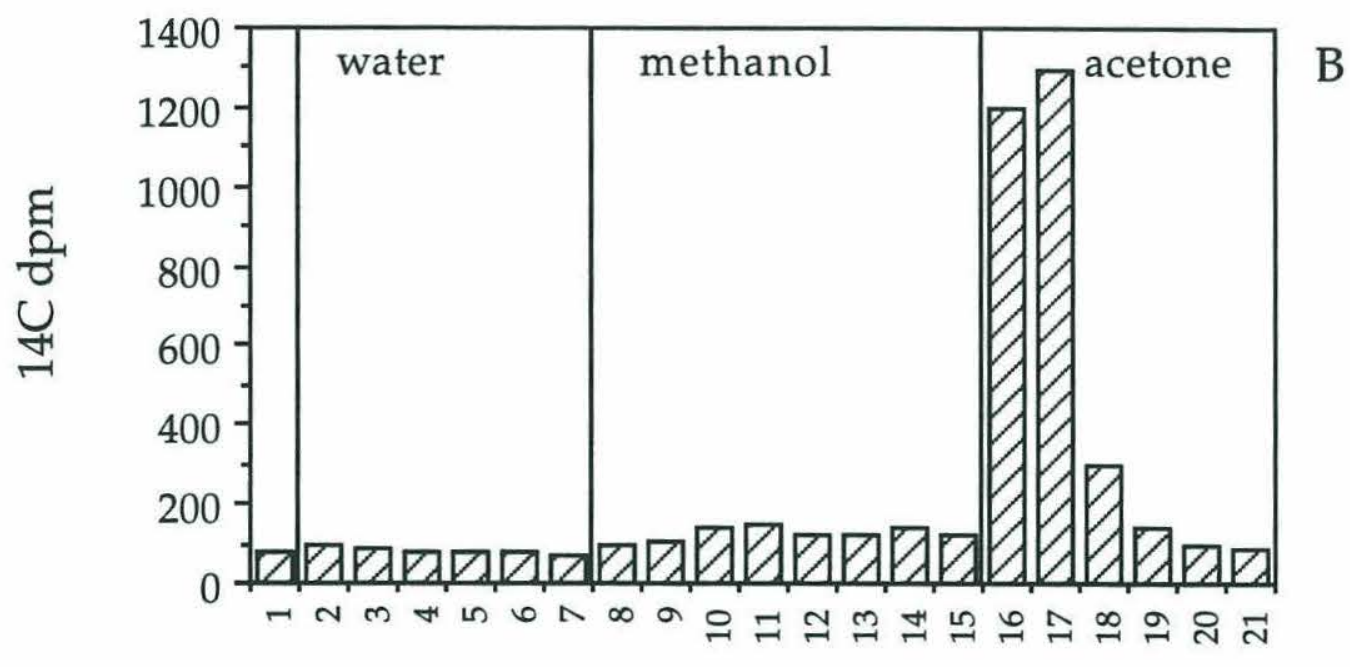

Fraction \# $(1 \mathrm{ml})$

Figure 7. XAD column profiles of 3,3',4,4'-TCB metabolites produced in microsomes from a male beluga whale (A), and of 3,3',4,4'-TCB parent compound (B). Metabolites were generated and separated on the column as described in Methods. Bars represent the radioactivity contained in each $1 \mathrm{ml}$ fraction, eluted from the column as it was washed with $1 \mathrm{ml}$ aliquots of water, methanol, and acetone. The first three (A) or first one (B) fractions are $\mathrm{H}_{2} \mathrm{O}$ column blanks, the next fraction was eluted as sample was applied to the column. 
suggests the formation of two classes of TCB metabolites, with different degrees of aqueous solubility.

\section{Discussion}

Cetaceans throughout the world have been found to contain chlorobiphenyls and other polyhalogenated aromatic hydrocarbons in their tissues. Inferences have been made regarding disposition of these compounds, based on relative concentrations found in cetacean and other environmental samples. However, there is no quantitative information on the capacity of these animals to transform and eliminate such compounds. In the case of protected species like marine mammals, the study of in vitro pollutant metabolism is one of the few tools available to assess this capacity. We have quantified the rates of metabolism of two tetrachlorobiphenyl congeners by hepatic microsomes from beluga whale and pilot whale. The rates of metabolism of 3,3',4,4'-TCB in beluga samples were comparable to those reported in induced rat liver (see below), whereas the rates of this activity in pilot whale samples were comparatively slow. We report a moderate rate of metabolism of $2,2^{\prime}, 5,5^{\prime}$-TCB in both cetacean species. The production of polar TCB derivatives in vitro suggests the capacity for biotransformation of TCBs in vivo, which would allow for detoxification and elimination, but may also lead to the production of toxic or reactive metabolites.

\section{Monooxygenase systems}

The values reported here for pilot whale cytochromes P450 and b5 and for EROD activity are similar to those reported for other cetacean species, specifically beluga whale (White et al., 1994), minke whale (Goksøyr et al., 1986; Goksøyr et al., 1988), and short-finned pilot whale, striped dolphin, and killer whale (Watanabe et al., 1989). The strong positive correlation between CYP1A content and EROD activity (Fig 2) suggests that a CYP1A form is the principal catalyst of this activity in pilot whale, as is generally the case in mammals. We speculate that expression of CYP1A in pilot whale may be related to body burden of Ah receptor agonists, as it is in beluga (White et al., 1994). The pilot whales stranded in December 1990 had significantly greater CYP1A content and EROD activity than did the individuals stranded in September-October, 1991. Because of the small sample size and the large spread in values, this inter-group difference may be due to statistical sampling error (i.e. chance differences, when randomly sampling from two similar populations). However, the inter-group difference also suggests that the two populations had different exposure histories. Pilot whales from the western North Atlantic 
have been found to accumulate appreciable concentrations of DDTs, PCBs, chlordanes, and other chlorinated organics (Muir et al., 1988), some of which are potential CYP1A inducers. Exposure to such compounds would depend both upon geographic range, and on contaminant residues in prey populations. The two groups of pilot whales in this study were stranded on different months and in separate years, suggesting that differential exposure (particularly differential recent exposure) was possible.

\section{$\underline{3,3^{\prime}, 4,4^{\prime}-\mathrm{TCB} \text { metabolism }}$}

The rates of metabolism of 3,3',4,4'-TCB in male beluga hepatic microsomes were similar to rates reported in hepatic microsomes from rats treated with CYP1A inducers: 18 and 50 $\mathrm{pmol} / \mathrm{min} / \mathrm{mg}$ in MC-treated rats (Mills et al., 1985; Ishida et al., 1991), and 22 $\mathrm{pmol} / \mathrm{min} / \mathrm{mg}$ in rats treated with Aroclor 1254 (Borlakoglu and Wilkins, 1993). In an earlier study (White et al., 1994) we provided evidence indicating that hepatic CYP1A in these beluga was induced by exposure to environmental contaminants, including coplanar PCBs. Such induction may explain the elevated rates of TCB metabolism in these beluga. The strong sex difference in rate of 3,3',4,4'-TCB metabolism is consistent with sex differences in CYP1A content and in blubber concentration of coplanar PCBs in this population (White et al., 1994). It should be noted, however, that these "high" rates of TCB metabolism are still one order of magnitude slower than the rates of metabolism of benzo[a]pyrene in hepatic microsomes of the same individuals (White et al., 1994).

The pilot whale samples selected for TCB metabolism analysis had very limited capacity to transform 3,3',4,4'-TCB, in comparison to rat and beluga. Despite apparently elevated CYP1A content and EROD activity in the individuals stranded in 1990, the rates of metabolism of 3,3',4,4'-TCB in this group were only slightly higher than rates in the 1991 group. The low rates in the 1990 group may be related to sample deterioration, as these animals were not dissected until some hours after death (see Methods). However, we doubt that to be the case, since rates of EROD activity were relatively high in the 1990 samples. It is possible that the two cetacean species simply possess different capacities for this activity, which may involve differences in CYP1A or differences in the contributions of non-CYP1A catalysts to this activity (see below). Similar levels of CYP1A expression do not bring about similar rates of 3,3',4,4'-TCB metabolism in the two cetacean species: rate of TCB metabolism per nmol CYP1A was far lower in the pilot whales (Table 2), despite the fact that rate of EROD activity per nmol CYP1A was similar between the two species.

Our data suggest that CYP1A is a principal catalyst for the metabolism 3,3',4,4'TCB in cetaceans, as has been shown in rats (Shimada and Sawabe, 1983; Mills et al., 
1985; Ishida et al., 1991). The rate of metabolism of 3,3',4,4'-TCB by each cetacean species was positively correlated with immunodetectable CYP1A content and with rate of CYP1A-mediated EROD activity (Fig 4), indicating that CYP1A contributes to this activity in cetaceans. Inhibition studies with beluga liver microsomes (Figs 5 and 6) further indicate a role for CYP1A in the metabolism of 3,3',4,4'-TCB. In the first inhibition study, EROD activity was inhibited almost $100 \%$ by $1 \mu \mathrm{M}$ ANF, $16 \%$ by $1 \mu \mathrm{M} 3,3^{\prime}, 4,4^{\prime}-$ TCB, and $0 \%$ by $1 \mu \mathrm{M} 2,2^{\prime}, 5,5^{\prime}-\mathrm{TCB}$. This result suggests that 3,3',4,4'-TCB has some ability to bind to and inhibit the beluga EROD catalyst (CYP1A), although such binding appears weak by comparison to ER or ANF binding. 3,3',4,4'-TCB appears to be a weaker EROD inhibitor in hepatic microsomes of beluga than in hepatic microsomes of other species studied. The Ki for EROD inhibition by 3,3',4,4'-TCB has been determined to be $15 \mathrm{nM}$ in rat, $15 \mathrm{nM}$ in chick embryo (De Matteis et al., 1989), and $20 \mathrm{nM}$ in the marine fish scup (Hahn, unpublished observation). Although we did not determine a Ki for beluga, it would appear to be in excess of $1 \mu \mathrm{M} 3,3^{\prime} 4,4^{\prime}-\mathrm{TCB}$. This result may suggest a cetacean CYP1A of slightly different structure, reflecting the divergent evolution of marine mammals. This question will ultimately be addressed by sequencing the beluga CYP1A gene and comparing to sequences of animals from other classes and orders.

In the second inhibition study, ANF inhibited EROD activity by almost $100 \%$, $3,3^{\prime}, 4,4^{\prime}-$ TCB metabolism by up to $47 \%$, and $2,2^{\prime}, 5,5^{\prime}$-TCB metabolism by $0-15 \%$. ANF is a strong inhibitor of CYP1A catalytic activity in mammals, but recent evidence indicates that it is not entirely specific for inhibition of CYP1A forms (Chang et al., 1994). Therefore, an approximately $50 \%$ inhibition of cetacean 3,3',4,4'-TCB metabolism by ANF suggests that as much as $50 \%$ of this activity is catalyzed by CYP1A. In contrast to these data, Ishida and co-workers (Ishida et al., 1991) found that the metabolism of $3,3^{\prime}, 4,4^{\prime}-\mathrm{TCB}$ in hepatic microsomes from MC-treated rat was inhibited $78 \%$ by $10 \mu \mathrm{M}$ ANF, and $86 \%$ by $100 \mu \mathrm{M}$ ANF. Our data may be interpreted in two ways: (1) more than one catalyst contributes to the metabolism of 3,3',4,4'-TCB in beluga liver microsomes, or (2) beluga CYP1A displays binding and catalytic properties toward ANF, TCB, and ER, which differ significantly from CYP1A in other species studied.

\section{$\underline{2,2}, 5,5^{\prime}-\mathrm{TCB}$ metabolism}

Distinct from the species difference in capacity for metabolism of 3,3',4,4'-TCB, the capacity to metabolize $2,2^{\prime}, 5,5^{\prime}$-TCB was approximately equal in the beluga and pilot whale samples. Thus the enzyme(s) responsible for 2,2',5,5'-TCB metabolism appear to be regulated independently of the principal enzyme(s) that catalyze 3,3',4,4'-TCB metabolism in these species. CYP1A appears to play little or no role in this activity, 
indicated by the finding that 2,2',5,5'-TCB does not inhibit EROD activity and by the finding that ANF does not strongly inhibit 2,2',5,5'-TCB metabolism.

The rates that we measured for 2,2',5,5'-TCB metabolism in cetacean liver microsomes are lower than some that have been found in control rat liver microsomes: 2.3 $\mathrm{pmol} / \mathrm{min} / \mathrm{mg}$ (our laboratory), and $10 \mathrm{pmol} / \mathrm{min} / \mathrm{mg}$ (Ishida et al., 1991). Other investigators, however, have reported no detectable activity in control rat liver microsomes (Ghiasuddin et al., 1976; Mills et al., 1985). In hepatic microsomes from PB-treated rat, rates ranging from 4 (Ghiasuddin et al., 1976) to 66 (Mills et al., 1985) to 1450 $\mathrm{pmol} / \mathrm{min} / \mathrm{mg}$ (Ishida et al., 1991) have been reported.

Studies with rodents indicate that PB-inducible CYP2B enzymes are principal catalysts for the metabolism of non-coplanar PCB congeners, including 2,2',5,5'-TCB (Shimada et al., 1981; Mills et al., 1985; Ishida et al., 1991). However, the expression and activity of CYP2B forms in cetacean liver is still open to question. Catalytic data, including PROD activity in beluga (White et al., 1994), aldrin epoxidase activity in shortfinned pilot whale, striped dolphin, and killer whale (Watanabe et al., 1989), and metabolism of 2,2',5,5'-TCB in pilot whale and beluga (this study) suggest that CYP2Blike activities are slight in cetaceans. Similarly, inferences based on PCB residue data from cetaceans suggest very low CYP2B-like activity. Tanabe et al. (Tanabe et al., 1988) found selective enrichment of meta-para unsubstituted PCB congeners in Dall's porpoise and striped dolphin, and Norstrom et al. (Norstrom et al., 1992) found selective enrichment of these congeners in Arctic beluga, suggesting comparatively slow metabolism and depuration of those congeners that are CYP2B substrates in rat.

Despite the catalytic data, immunochemical results indicate the presence of a possible CYP2B homologue in liver microsomes of pilot whale and beluga whale. This protein (or proteins) has an electrophoretic migration near that of cytochromes $\mathrm{P} 450$ and is immunochemically related to rabbit CYP2B4 and dog CYP2B10. However, the lack of cross-reaction with antibodies specific to rat CYP2B1/2B2 raises questions about the identity of the cetacean protein(s). It is not yet clear whether these proteins bear structural or functional similarity to CYP2B forms in other mammals. It remains an interesting possibility that the cetacean protein(s) detected are catalysts of 2,2',5,5'-TCB metabolism in these species.

\section{$\underline{\text { PCB residues }}$}

Our in vitro data complement $\mathrm{PCB}$ residue data in providing a direct demonstration of selective PCB metabolism and elimination by cetaceans. Several studies have noted selective retention in cetacean blubber of those PCBs resembling CYP2B-like substrates 
(i.e. meta-para unsubstituted congeners), with selective elimination from blubber of those PCBs resembling CYP1A-like substrates (Tanabe et al., 1988; Duinker et al., 1989; Norstrom et al., 1992). We report very slow rates of metabolism in beluga liver microsomes of 2,2'5,5'-TCB, a meta-para unsubstituted congener that resembles those congeners that tend to accumulate in cetacean blubber. We report a much higher rate of metabolism in beluga liver microsomes of 3,3',4,4'-TCB, a CYP1A-like substrate that belongs to the group of congeners that are selectively eliminated from cetacean tissue.

There are several routes by which PCBs may be eliminated from mammalian tissue, including metabolism, loss of parent compound to urine and feces, and transfer through lactation and pregnancy. The rates of PCB elimination via non-metabolic routes are generally either not congener-specific, or are dependent upon congener lipophilicity and thus on chlorine number. For example, PCB elimination by Dall's porpoise during parturition and lactation was greater for the lower chlorinated congeners than for the higher (Subramanian et al., 1988), half-lives for PCB elimination from exposed humans were shorter for tetra- and penta- than for hexa- and hepatchlorobiphenyls (Ryan et al., 1993), and rates of PCB depuration from fish have generally been found to increase with decreasing chlorine number and with decreasing octanol/water partition coefficient (Niimi and Oliver, 1983; Jones et al., 1989). A differential elimination of two congeners with the same chlorine number (e.g. 2,2',5,5'-TCB and 3,3',4,4'-TCB) is likely to be mediated by differential rates of metabolism in vivo. Our in vitro data support a model in which rates of metabolism, and consequent elimination, govern the relative concentrations of some PCB congeners in cetacean tissue.

In summary, we have provided the first direct demonstration of polychlorinated biphenyl metabolism in marine mammals. Catalytic and immunochemical data suggest that PCB metabolism in these cetacean species may follow similar structure-activity relationships as those found in rat, with CYP1A being involved in coplanar PCB metabolism and CYP2B involved in noncoplanar PCB metabolism. Our data suggest that CYP1A induction (seen in these beluga) may enhance the metabolism of the toxic, coplanar PCB congeners by cetaceans, but that metabolism of the noncoplanar congeners is limited. Furthermore, these in vitro results, coupled with residue data, provide evidence for the importance of PCB metabolism to cetaceans that are exposed to these environmental pollutants. This study provides a framework for further investigation of the capacity of marine mammals to biotransform lipophilic organic contaminants, and of the effects of contaminants and their metabolites on marine mammals. 


\section{CHAPTER SEVEN}

Catalytic and Immunochemical Characterization of Hepatic Microsomal

Cytochromes P450 in Beluga Whale (Delphinapterus leucas)

Reprint from Toxicology and Applied Pharmacology 126 
Reprinted with permission from Academic Press, Inc.

\title{
Catalytic and Immunochemical Characterization of Hepatic Microsomal Cytochromes P450 in Beluga Whale (Delphinapterus leucas) ${ }^{1}$
}

\author{
Renee D. White, * Mark E. Hahn, ${ }^{*}$ W. Lyle Lockhart, $\dagger$ and John J. Stegeman ${ }^{*, 2}$ \\ *Biology Department, Redfield 3-42. Woods Hole Oceanographic Institution, Woods Hole, Massachusetts 02543; and †Freshwater Institute. \\ Department of Fisheries and Oceans, Winnipeg R3T 2N6, Canada
}

Received August 23, 1993; accepted December 17, 1993

Catalytic and Immunochemical Characterization of Hepatic Microsomal Cytochromes P450 in Beluga Whale (Delphinapterus leucas). WHITE, R. D., HAHN, M. E., LOCKHART, W. L., and Stegeman, J. J. (1994). Toxicol. Appl. Pharmacol. 126, 45-57.

Understanding the effects of environmental contaminants on cetaceans and other marine mammals will require information on the biochemistry of xenobiotic metabolism in these species. We characterized the hepatic microsomal cytochrome P450 system in beluga whales (Delphinapterus leucas) from the Canadian Arctic. The content of native P450 averaged 0.203 and $0.319 \mathrm{nmol} / \mathrm{mg}$ microsomal protein, cytochrome b5 content averaged 0.199 and $0.236 \mathrm{nmol} / \mathrm{mg}$, and rates of NADPH-cytochrome $\mathrm{c}$ reductase were 79 and $76 \mathrm{nmol} / \mathrm{min} / \mathrm{mg}$, for females and males respectively. Ethoxyresorufin O-deethylase (EROD), pentoxyresorufin O-depentylase (PROD), and benzo[a]pyrene (BP) hydroxylase (AHH) activities were significantly greater in males than in females, and were highly correlated with one another ( $r^{2}$ between 0.853 and 0.912 ). HPLC analysis of in vitro BP metabolites revealed benzo-ring (7,8- and 9,10-) dihydrodiols, consistent with activation of this compound, as well as 4,5-dihydrodiol, 3-OH-, 7-OH-, and 9-OH-BP and 1,6- and 3,6quinones. Estradiol 2-hydroxylase activity did not differ between sexes, and rates did not correlate with those of the other activities. Antibodies against scup P450B (an apparent teleost CYP2B) and rat CYP2B1 did not recognize proteins in beluga liver microsomes, but there was a protein detected by antibodies to PB-inducible rabbit CYP2B4. Antibodies to ethanol and ketone-inducible rat CYP2E1 reacted with two proteins in beluga liver microsomes. Antibodies specific to hydrocarbon-inducible CYP1A1 and/or CYP1A2 forms showed a single protein band, apparently more closely related to CYPIA1. The content of CYP1A was fivefold greater in male than in female beluga. CYP1A content was highly correlated with EROD, PROD, and AHH activities, suggesting that this $\mathrm{P} 450$ form is a primary catalyst for these reactions in beluga. CYP1A content and activity were highly correlated with the concentrations in blubber of non-ortho and mono-ortho PCB congeners, compounds that induce CYP1A in other mammals. These results indicate that a CYP1A is a catalyst for the metabolism of aromatic hydrocarbon pollutants in the beluga whale, and strongly suggest that this protein is induced in these organisms by environmental contaminants, including PCBs. The results support the measure- ment of CYP1A expression as a biomarker of exposure to inducers in marine mammals. The full functional and evolutionary relationships of beluga CYP1A and of beluga proteins immunologically related to other P450 forms are uncertain. - 1994 Academic Press, Inc.

Many marine mammals accumulate appreciable concentrations of organic pollutants, including polycyclic aromatic hydrocarbons (PAH) ${ }^{3}$ chlorinated pesticides, polychlorinated biphenyls (PCB), and other halogenated aromatic hydrocarbons (Ono et al., 1987; Aguilar and Borrell, 1988; Muir et al., 1988; Duinker et al., 1989; Hellou et al., 1991; Woodley et al., 1991). Their large lipid reservoirs may strongly contribute to retention of lipophilic xenobiotics, and thus to contaminant accumulation. It is speculated that such contaminants exert effects on marine mammals at the biochemical, organismal, and perhaps even the population levels, but few studies have addressed these effects in a detailed way (Engelhardt, 1982; Reijnders, 1986). The odontocetes (toothed whales) may be a particularly vulnerable group, as they are top predators in oceanic ecosystems and appear to bioaccumulate pollutants from their prey organisms.

Among the odontocetes, the beluga whale (Delphinapterus leucas) is a species of particular interest and concern. The population of beluga inhabiting the St. Lawrence Estuary is one of the most heavily contaminated groups of

\footnotetext{
1 This research was supported in part by NIH Grants ES04220 (J.J.S.) and ES05479 (M.E.H.), by EPA Grant R817988, by the WHOI/MIT Joint Doctoral Program (R.D.W.), and by the Stanley W. Watson Chair (J.J.S.). Presented in part at the 1992 Annual Meeting of the Society of Environmental Toxicology and Chemistry, Cincinnati, Ohio.

${ }^{2}$ To whom correspondence should be addressed.

${ }^{3}$ Abbreviations used: EROD, ethoxyresorufin O-deethylase; PROD, pentoxyresorufin O-depentylase; $\mathrm{BP}$, benzo[a]pyrene; $\mathrm{AHH}$, aryl hydrocarbon hydroxylasc; HPLC, high-performance liquid chromatography; PB, phenobarbital; $\mathrm{PCB}$, polychlorinated biphenyl; $\mathrm{PAH}$, polycyclic aromatic hydrocarbon; $E_{2}-2 \mathrm{OH}$, estradiol 2-hydroxylase; ANF, alpha naphthoflavone; MAb, monoclonal antibody; MO, monooxygenase; DHD, dihydrodiol.
} 


\section{WHITE ET AL.}

marine mammals known (Masse et al., 1986; Martineau et al., 1987; Muir et al., 1990), having high concentrations of PCB, DDT, and other organochlorine pesticides. Such contamination has been suggested as a causative factor in the failure of this population to recover from the hunting-related population decline earlier in this century (Martineau et al., 1987, 1988). At present there is no information on the biochemistry of xenobiotic metabolism and its role in determining the susceptibility or resistance to pollutants in this species. A biochemical and molecular characterization of microsomal enzymes could provide a better understanding of the effects of environmental pollutants on beluga, and on cetaceans in general.

Cytochrome P450 monooxygenases are central to the oxidative metabolism of a wide array of endogenous and xenobiotic compounds (Nebert and Gonzalez, 1987), including many marine pollutants. P450-mediated metabolism can result in either the detoxification or the activation of xenobiotic substrates, thus determining toxicity. Members of the P450 subfamilies CYP1A, CYP2B, and CYP2E (Nelson et al., 1993) are important in metabolism of many pollutants. CYP1A forms are inducible by $\mathrm{PAH}$ and by planar, polyhalogenated aromatic compounds (e.g., PCBs, chlorinated dioxins), which may then be metabolized by these enzymes. Proteins in the CYP2B subfamily are inducible in terrestrial mammals by barbiturates and by ortho-substituted (noncoplanar) polyhalogenated biphenyls, which in turn may be metabolized by these enzymes.

A limited number of previous studies have described P450 or P450-associated activities in pinnipeds (Engelhardt, 1982; Addison and Brodie, 1984; Addison et al., 1986; Goksøyr et al., 1992) and cetaceans (minke whale, pilot whale, killer whale, and dolphin) (Goksøyr et al., 1986, 1988; Watanabe et al., 1989). Other studies have inferred P450 characteristics based on patterns of PCB congeners detected in cetacean tissues, relative to those found in the environment (Tanabe et al., 1988; Duinker et al., 1989; Norstrom et al., 1992). For example, based on the relative persistence of meta-para unsubstituted PCB congeners in cetacean tissue, it has been suggested that monooxygenase activities characteristic of CYP2B are reduced in cetaceans compared to those in terrestrial mammals (Tanabe et al., 1988; Norstrom et al., 1992). Other workers have reported aromatic DNA adducts, ostensibly of $\mathrm{PAH}$, in the livers of Arctic beluga (Ray et al., 1991), suggesting bioactivation of such compounds. In this study we describe the hepatic P450 system in beluga from a site in the Canadian Arctic distant from known inputs of pollutants. Results of our catalytic and immunochemical assays indicate that a hepatic microsomal CYP1A isoform is expressed in beluga whale. The content of this protein is correlated with the content of chlorobiphenyl residues, indicating that hepatic CYP1A has been induced by environmental chemicals in these Arctic beluga, and providing a basis of comparison with other cetacean populations, including beluga from the contaminated St. Lawrence system. Our results also indicate that expression of CYP2B-related proteins is uncertain in these whales. Cetaceans, which diverged from the ungulate line, may provide a valuable point of comparison for establishing the fundamental roles and evolution of P450 enzymes in mammalian species.

\section{MATERIALS AND METHODS}

Chemicals. All chemicals used were of the highest grade available from commercial suppliers and were similar to those used previously in this laboratory (Stegeman and Binder, 1979). 7-Ethoxyresorufin was synthesized as previously described (Klotz et al., 1984). Benzo[a]pyrene was purified by extraction with $0.15 \mathrm{~N} \mathrm{KOH}$ in $85 \%$ dimethyl sulfoxide. Estradiol was purified by thin-layer chromatography in a solvent system consisting of chloroform:acetone at a ratio of 7:3. Antibodies used are described below.

Tissues. Beluga whales (D. leucas) from the Husky Lakes of the Tuktoyuktuk peninsula, on the MacKenzie River delta (Canadian Arctic), were hunted by local Inuit in a subsistence harvest in November, 1989. Freshly killed individuals were weighed and measured and sexes were determined. On average, these animals weighed $200 \mathrm{~kg}$ less than expected for beluga of their length (Lockhart $e t$ al., in preparation), suggesting that they had not fed for a period prior to capture. Liver samples were removed within $30 \mathrm{~min}$ of termination. Slices of liver $0.5-$ to $1.0-\mathrm{cm}$ thick (approximately $50 \mathrm{~g}$ per animal) were frozen immediately on dry ice and then stored at $-80^{\circ} \mathrm{C}$ until microsomes were prepared. Portions of liver from 13 animals were obtained from the Canadian Department of Fisheries and Oceans.

Preparation of cytosol and microsomes. Pieces of frozen liver were pulverized at liquid nitrogen temperatures with a ceramic mortar and pestle. Liver powders were homogenized with a Teflon-glass homogenizer (10 passes) in 9 vol of cold $25 \mathrm{mM}$ MOPS buffer, $\mathrm{pH} 7.5$, at $20^{\circ} \mathrm{C}$, containing 1 mM dithiothreitol, $1 \mathrm{~mm}$ EDTA, $5 \mathrm{~mm}$ EGTA, 0.02\% $\mathrm{NaN}_{3}, 20 \mathrm{~mm}$ $\mathrm{Na}_{2} \mathrm{MoO}_{4}, 10 \%$ (v:v) glycerol, and a mixture of protease inhibitors $(20 \mathrm{mM}$ tosyl L-lysine chloromethyl ketone, $5 \mathrm{mg} / \mathrm{ml}$ leupeptin, $13 \mathrm{mg} / \mathrm{ml}$ aprotinin, $7 \mathrm{mg} / \mathrm{ml}$ pepstatin $\mathrm{A}$, and $0.1 \mathrm{~mm}$ phenylmethylsulfonylfluoride). This homogenization buffer was designed to yield microsomal fractions and cytosolic fractions suitable for Ah receptor assays (Hahn et al., 1994). Homogenates were centrifuged for $10 \mathrm{~min}$ at $750 \mathrm{~g}$ and $10 \mathrm{~min}$ at $12,000 \mathrm{~g}$. The supernate was then centrifuged at $100,000 \mathrm{~g}$ for $70 \mathrm{~min}$. The supernatant fraction (cytosol) was removed and frozen in liquid $\mathrm{N}_{2}$. The $100,000 \mathrm{~g}$ pellet was resuspended in a buffer that was $0.1 \mathrm{M}$ Tris, $\mathrm{pH} 7.6,1 \mathrm{mM}$ EDTA, $1 \mathrm{mM}$ DTT, and $20 \%$ glycerol, at $2 \mathrm{ml} / \mathrm{g}$ liver, and frozen in liquid $\mathrm{N}_{2}$. Protein was measured by the bicinchoninic acid method (Smith et al., 1985).

Cytochromes P450 and b5. The content of microsomal cytochrome P450 was determined from dithionite difference spectra of CO-treated samples (Stegeman and Binder, 1979), assuming extinction coefficients of 91 $\mathrm{mM}^{-1} \mathrm{~cm}^{-1}$ for P450 and $111 \mathrm{~mm}^{-1} \mathrm{~cm}^{-1}$ for P420 (Omura and Sato, 1964). There were large peaks of absorbance at $420 \mathrm{~nm}$ in some samples. Therefore, samples were treated with ascorbic acid and phenazine methosulfate (Johannesen and DePierre, 1978) to evaluate hemoglobin content. Cytochrome b5 was measured using NADH difference spectra, as described previously (Stegeman and Binder, 1979).

Enzyme assays. All assays were conducted at $37^{\circ} \mathrm{C}$. NADPH cytochrome c (P450) reductase activity was measured by monitoring absorbance at $550 \mathrm{~nm}$, as previously described (Stegeman et al., 1979). Estradiol 2-hydroxylase activity ( $\mathrm{E}_{2}-2 \mathrm{OHase}$ ) was assayed by ${ }^{3} \mathrm{H}_{2} \mathrm{O}$ release from [2${ }^{3} \mathrm{H}$ ] estradiol (Snowberger and Stegeman, 1987). Ethoxyresorufin O-deethylase (EROD) activity was quantified using a spectrophotometric kinetic 
assay (Klotz et al., 1984). Pentoxyresorufin O-depentylasc (PROD) activity was similarly assayed by measuring resorufin formation, using the spectrophotometric assay as described for EROD activity (Klotz et al., 1984). PROD activity was also assayed fluorometrically as follows: The $500-\mu$. reaction mixture contained $0.1 \mathrm{M}$ Tris, $\mathrm{pH} 8,0.1 \mathrm{M} \mathrm{NaCl}$, 7-pentoxyresorufin at a nominal concentration of $5 \mu \mathrm{M}$ (dissolved in dimethyl sulfoxide), and $60-100 \mu \mathrm{g}$ of microsomal protein. Reactions were initiated with $10 \mu$ NADPH $(25 \mathrm{mg} / \mathrm{ml})$, allowed to run for $15 \mathrm{~min}$ at $37^{\circ} \mathrm{C}$, and stopped with $1 \mathrm{ml}$ of cold methanol. These were centrifuged at low speed, and resorufin fluorescence in the supernatants was determined using an SLM Aminco SPF-500 spectrofluorometer. Excitation was at $550 \mathrm{~nm}$ and fluorescence emission was measured at $588 \mathrm{~nm}$, which was the wavelength of maximum emission for the resorufin in these samples. Resorufin content was quantified from a standard curve, generated by measuring the fluorescence of known amounts of resorufin in the above buffer.

Benzo[a]pyrene (BP) hydroxylase (aryl hydrocarbon hydroxylase; $\mathrm{AHH}$ ) activity was measured with a radiometric assay (Binder and Stegeman, 1980), and samples were counted in a Beckman LS5000TD liquid scintillation counter. Inhibition of $\mathrm{AHH}$ activity by alpha-naphthoflavone (ANF) was determined by adding $10^{-4} \mathrm{M}$ ANF dissolved in methanol to the assay tubes (total volume $1 \mu \mathrm{l}$ methanol per $50-\mu \mathrm{l}$ assay).

Benzola|pyrene metabolites. Benzo[a]pyrene metabolites were generated in vitro from $\left[{ }^{3} \mathrm{H}\right] \mathrm{BP}$, using reaction mixtures similar to those for AHH quantitation. Reaction mixtures of $500 \mu$ l were incubated for 20 min at $37^{\circ} \mathrm{C}$, stopped with $1 \mathrm{vol}$ of acetone, and extracted twice with $2 \mathrm{ml}$ ethy] acetate, as previously described (Stegeman et al., 1984). The solvent layer was dried under $\mathrm{N}_{2}$ gas and resuspended in methanol. Prior to extraction, 5 $\mu \mathrm{g}$ of $6 \beta$-hydroxytestosterone was added to each sample as an internal standard.

BP metabolite profiles were obtained by high-performance liquid chromatography, using a Rainin Dynamax C- 18 column $(4.6 \times 250 \mathrm{~mm}, 5 \mu \mathrm{m})$ and a Hewlett-Packard diode array detector. Metabolites were eluted with a water/methanol/ethanol gradient solution, at a flow rate of $1 \mathrm{ml} / \mathrm{min}$, as follows: start at $45 \%$ water, $37 \%$ methanol, $18 \%$ ethanol; 15 -min transition to $35 \%$ water, $44 \%$ methanol, $21 \%$ ethanol; $25-\mathrm{min}$ transition to $25 \%$ water, $50 \%$ methanol, $25 \%$ ethanol; immediate change to $67 \%$ methanol, $33 \%$ ethanol: hold $20 \mathrm{~min}$. Metabolites were identified by comparison of retention times and uv/visible absorption spectra with those of authentic standards. Metabolites were quantified by collecting fractions eluting from the column and measuring radioactivity in a Beckman LS5000TD scintillation counter.

Immunoblotting. Immunoblot ("Western" blot) analysis was performed as described previously (Kloepper-Sams et al., 1987; Stegeman et al., 1991). Primary antibodies used were as follows: monoclonal antibody 1-12-3, raised against scup cytochrome P450E (CYPIA1) (Park et al., 1986); a polyclonal antibody raised against mouse CYPIAI but recognizing both CYPIA1 and CYPIA2 forms, provided by Dr. Peter Sinclair (Veterans Administration Medical Center, White River Junction, VT); a polyclonal antibody against scup cytochrome P450B (Gray, 1988), which is likely a CYP2B form (Stegeman et al., 1990); anti-P450PB4, a polyclonal antibody against rat CYP2B1, provided by Dr. David Waxman (DanaFarber Cancer Institute, Boston, MA) (Frey et al., 1985); MAb 4-29-5 p4, a monoclonal antibody against rat CYP2B I, provided by Dr. Harry Gelboin (N1H, Bethesda, MD) (Park et al., 1984); anti-P450LM2, a goat polyclonal antibody against rabbit CYP2B4, obtained from Oxford Biomedical Research, Inc. (Oxford, MI); anti-dog CYP2BII, provided by Dr. James Halpert (University of Arizona) (Duignan et al., 1987); anti-P450RLM6, a polyclonal antibody to rat CYP2EI, provided by Dr. John Schenkman (University of Connecticut Health Center, Farmington, CT) (Favreau et al. 1987); and a polyclonal antibody against rat CYP2E1 obtained from OXYGENE Dallas (Dallas, TX). Secondary antibodies used were alkaline phosphatase linked goat-anti-mouse IgG, goat-anti-rabbit IgG, and rabbitanti-goat IgG. Content of the P450E homologue (CYPIA) in beluga microsomes was quantified by densitometric scanning of MAb 1-12-3-reac-

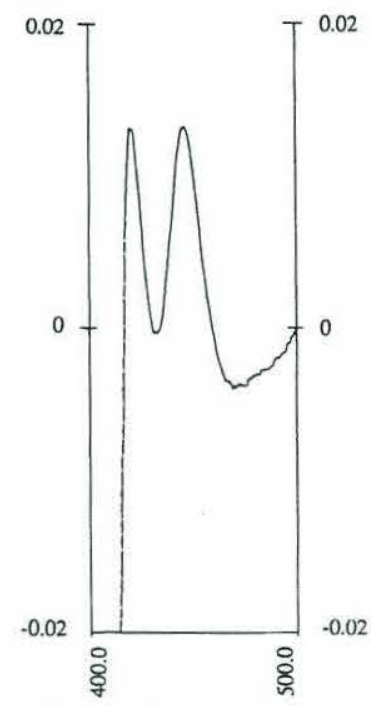

FIG. 1. Dithionite difference spectrum of beluga liver microsomes Data were obtained by measuring the dithionite difference spectrum of CO-treated microsomes as described under Materials and Methods. Microsomal protein concentrations in the assay were 0.6 to $1.0 \mathrm{mg} / \mathrm{ml}$. y axis, absorption; $x$ axis, wavelength $(\mathrm{nm})$.

tive bands with a video imaging system, and comparison to P450E standards. Because the strength with which MAb 1-12-3 recognizes P450E homologues may differ between species, CYPIA levels deduced from the immunoblot represent relative rather than absolute values for this species.

Statistical analyses. A pooled, two-tailed $t$ test was used to determine significant differences between male and female sample groups. The twotailed Smith-Satterthwaite $t$ test was used to compare male and female groups in those cases in which the variances for the two groups were very different, which included EROD, PROD, and AHH activities and CYPIA content.

\section{RESULTS}

\section{Microsomal Electron Transport Components}

The dithionite difference spectra of CO-treated beluga hepatic microsomes showed a cytochrome $\mathrm{P} 450$ peak at $448 \mathrm{~nm}$ for all samples, and an additional peak at approximately $420 \mathrm{~nm}$ (Fig. 1). Ascorbic acid and phenazine methosulfate were added to some samples to determine whether the latter peak was due to the presence of oxyhemoglobin (Johannesen and DePierre, 1978); the resulting spectra indicated that hemoglobin could account for a minor percentage of the peak (data not shown). Thus, it appears that this peak primarily represents cytochrome $\mathrm{P} 420$. Such apparent degradation could occur during storage conditions prior to microsome preparation. NADH difference spectra were typical of cytochrome b5.

Average values for the hepatic content of native cy- 
WHITE ET AL.

TABLE 1

Microsomal Electron Transport Components in Beluga Whale Liver

\begin{tabular}{lcc}
\hline \multicolumn{1}{c}{ Component } & Female $(n=5)$ & Male $(n=8)$ \\
\hline Microsomal yield $(\mathrm{mg} / \mathrm{g} \mathrm{liver})$ & $14.5 \pm 0.5$ & $15.1 \pm 1.8$ \\
Cytochrome P450 $(\mathrm{nmol} / \mathrm{mg})$ & $0.203 \pm 0.063^{* *}$ & $0.319 \pm 0.073^{* *}$ \\
P450 + P420 $(\mathrm{nmol} / \mathrm{mg})$ & $0.843 \pm 0.224$ & $0.972 \pm 0.291$ \\
Cytochrome $\mathrm{b5}(\mathrm{nmol} / \mathrm{mg})$ & $0.199 \pm 0.027^{*}$ & $0.236 \pm 0.031^{*}$ \\
Reductase $(\mathrm{nmol} / \mathrm{min} / \mathrm{mg})^{\circ}$ & $79 \pm 7$ & $76 \pm 8$ \\
CYP1A $(\mathrm{pmol} / \mathrm{mg})^{b}$ & $9.9 \pm 7.1^{* * *}$ & $54.0 \pm 22.0^{* * *}$ \\
\hline
\end{tabular}

Note. Values are means $\pm \mathrm{SD}(n-1)$. Males and females are statistically different at a level ${ }^{*} p<0.05,{ }^{* *} p<0.02,{ }^{* * *} p<0.001$, based on a pooled $t$ test or a Smith-Satterthwaite $t$ test.

${ }^{a}$ Reductasc refers to NADPH cytochrome c (P450) reductase activity.

${ }^{b} \mathrm{CYP} 1 \mathrm{~A}$ is expressed as pmol P450E equivalents/mg.

tochrome P450, total cytochrome $\mathrm{P} 450$ (P450 + P420), cytochrome $\mathrm{b} 5$, and NADPH cytochrome $\mathrm{c}(\mathrm{P} 450)$ reductase are given in Table 1. Assuming a P420 extinction coefficient of $111 \mathrm{~mm}^{-1} \mathrm{~cm}^{-1}$, all samples contained a greater amount of P420 than P450. However, the precise extinction coefficient for this species has not been determined, and denaturation experiments with sodium dodecyl sulfate indicate that it may be considerably higher than $111 \mathrm{~mm}^{-1}$ $\mathrm{cm}^{-1}$ for these samples (data not shown). The values therefore may overestimate the actual P420 content. Nonetheless, the presence of $\mathrm{P} 420$ suggests that the catalytic rates measured here may not reflect the full capacity of the samples.

Values for native $\mathrm{P} 450$ content varied from 0.123 to $0.403 \mathrm{nmol} / \mathrm{mg}$; the mean value for males was significantly higher than that for females (Table 1). When combined with $\mathrm{P} 420$, the estimate of total P450 content approached $1.0 \mathrm{nmol} / \mathrm{mg}$, and the sex difference was no longer evident. Cytochrome b5 content and $\mathrm{P} 450$ reductase rates showed little variation about the means. A sex difference was seen in cytochrome b5 content but not in reductase activity.

\section{Monooxygenase Activities}

Rates of hepatic microsomal monooxygenase (MO) activities, EROD, $\mathrm{AHH}, \mathrm{PROD}$, and $\mathrm{E}_{2}-2 \mathrm{OHase}$, were measured in all samples and average values for males and females are listed in Table 2. Rates of PROD activity were too low to be detected by the spectrophotometric assay, but all samples gave quantifiable rates of resorufin formation when assayed fluorometrically. However, the rates in some samples were still near the limits of detection of the assay. To confirm that changes in fluorescence did reflect the appearance of resorufin, the product of several incubations was scanned over a range of wavelengths (Fig. 2). The resulting fluorescence spectra confirmed the presence of reso-
TABLE 2

Microsomal Monooxygenase Activities in Beluga Whale Liver

\begin{tabular}{lcc}
\hline \multicolumn{1}{c}{ Activity } & Female $(n=5)$ & Male $(n=8)$ \\
\hline EROD (pmol/min/mg) & $97 \pm 84^{* *}$ & $413 \pm 263^{* *}$ \\
PROD (pmol/min/mg) & $1.4 \pm 0.6^{* *}$ & $6.7 \pm 4.5^{* *}$ \\
$\mathrm{~F}_{2}-2 \mathrm{OH}(\mathrm{pmol} / \mathrm{min} / \mathrm{mg})$ & $56.9 \pm 18.0^{*}$ & $88.3 \pm 24.2^{*}$ \\
AHH (pmol/min/mg) & $74 \pm 41^{* *}$ & $264 \pm 158^{* *}$ \\
AHH with $10^{-4} \mathrm{M}$ ANF & & \\
$\quad(\%$ activity rcmaining) & $21 \pm 16 \%$ & $20 \pm 15 \%$ \\
EROD (pmol/min/nmol P450) & $451 \pm 373^{* * *}$ & $1255 \pm 691^{* * *}$ \\
PROD (pmol/min/nmol P450) & $7 \pm 3^{* *}$ & $20 \pm 12^{* *}$ \\
E $_{2}-2 \mathrm{OH}(\mathrm{pmol} / \mathrm{min} / \mathrm{mmol} \mathrm{P450)}$ & $283 \pm 412$ & $288 \pm 91$ \\
AHH (pmol/min/mmol P450) & $359 \pm 163^{* *}$ & $804 \pm 391^{* *}$ \\
\hline
\end{tabular}

Note. Statistical tests are as described in the legend to Table 1.

rufin, above background levels, even in samples with the lowest PROD activities.

Rates of all MO activities were significantly greater in males than in females, when expressed as activity per milligram of microsomal protein. EROD and PROD activities each varied over a 30 -fold range, yet for both the average of males was approximately four times that of females. AHH activity varied over a 15 -fold range, with a 3.5 -fold difference between males and females. Addition of $10^{-4} \mathrm{M}$ ANF, which is a strong and generally specific inhibitor of mammalian CYP1A in vitro (Testa and Jenner, 1981), caused a 53 to $100 \%$ inhibition of AHH activity. There was no difference in the degree of inhibition seen in male versus female

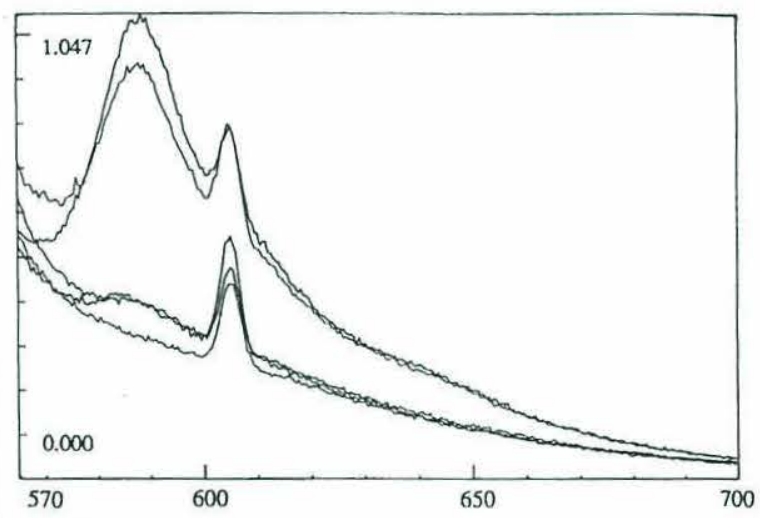

FIG. 2. Fluorescence spectra of pentoxyresorufin O-depentylase reaction mixtures. Bcluga liver microsomes were incubated with pentoxyresorufin as described under Materials and Methods, and the fluorescence spectrum was analyzed. Excitation was at $550 \mathrm{~nm}$. Resorufin peak occurs at $588 \mathrm{~nm}$. The lower curve is a blank incubation (without NADPH), the two middle curves are results from female beluga, and the two upper curves are from male beluga. Axes represent fluorescence $(y)$ versus wavelength $(x ; n m)$ 




FIG. 3. Immunoblot of heluga hepatic microsomes with $\mathrm{MAb}$ 1-12-3. Proteins were separated on an SDS-PAGE gel, transferred to nitrocellulose, and incubated with antibody raised against scup P450F (CYP1A1). Beluga microsomes were applied at $100 \mu \mathrm{g}$ protein/lane on a large gel. Lanes 1 and 14 are scup P450E. Lanes 2, 5, 7, 9, and 11 are fernale beluga. Lanes $3,4,6,8,10,12$, and 13 are male beluga. Lanes 3 and 8 are small males. Lane 10 is a male with low EROD activity and high microsomal P450. The lower-molecular-weight band seen in some lanes is likely a degradation product.

samples, nor was there any correlation found between rate of AHH activity and degree of inhibition by ANF. $\mathrm{E}_{2}$-2OHase activity per milligram microsomal protein varied over a 3.5-fold range, and the average rate in male samples was slightly but significantly higher than that in females.

Significant sex differences were still evident in rates of EROD, PROD, and AHH (Table 2) when activities were expressed per nanomole of native $\mathrm{P} 450$, an estimate of turnover number for the population of active P450 forms. Average $\mathrm{E}_{2}-2 \mathrm{OH}$ ase activity per nanomole enzyme was nearly identical for male and female samples.

\section{Immunodetection of Cytochrome P4501A}

Immunoblot analysis with MAb 1-12-3 revealed one major cross-reactive protein band in each beluga sample, with an electrophoretic migration suggesting a molecular weight slightly less than that of scup CYPIA1 (Fig. 3). This antibody, which was raised against scup P450E (CYP1A1), has been shown to strongly cross-react with mammalian CYPIAI, only weakly with CYP1A2, and not with any other microsomal proteins (Park et al., 1986; KloepperSams et al., 1987). To evaluate possible expression of an immunological counterpart to CYP1A2, samples were blotted with a polyclonal anti-mouse CYP1AI that recognizes both CYP1A1 and CYP1A2 in mice and rats (Peter Sinclair, personal communication). Only a single band was detected for each beluga (Fig. 4); the band was coincident in migration with the MAb 1-12-3-reactive band and the intensity of the band in different individuals was similar to that achieved with MAb 1-12-3. This may indicate that a single CYP1A gene is expressed in beluga. However, a second CYP1A form could exist which lacks the epitopes recognized by the antibodies used, which comigrates with the



FIG. 4. SDS-PAGE immunoblot of beluga hepatic microsomes with monoclonal anti-scup P450E (CYPIAI) (lanes 1 and 2) and polyclonal anti-mouse CYPIAI (lanes 2-7). Beluga protein loading was $100 \mu \mathrm{g} /$ lane, as in Fig. 3. Lane I, scup P450E. Lanes 2, 3, 4, malc bcluga. Lane 5, female beluga. Lane 6 , small male beluga. Lane 7 , liver microsomes ( $30 \mu \mathrm{g}$ protein) from a methylcholanthrene-treated rat. Molecular weights of the rat and scup enzymes determined by prior SDS-PAGE analysis are: rat CYPIAI, $56 \mathrm{kDa}$; rat CYPIA2, $52 \mathrm{kDa}$; Scup CYPIA1, $54 \mathrm{kDa}$. The cross-reactive protein in beluga has an apparent molecular weight of about $53 \mathrm{kDa}$.

1-12-3-reactive band, or which is simply not expressed in the individuals or the tissue examined here. Given this, and lacking sequence data, we refer to the beluga protein as CYPIA.

The content of the beluga CYP1A determined by immunoblot showed a highly significant sex difference (Table 1). Figure 5 illustrates the relationship of CYP1A content to sex and body length among the 13 animals (Fig. 5A), and
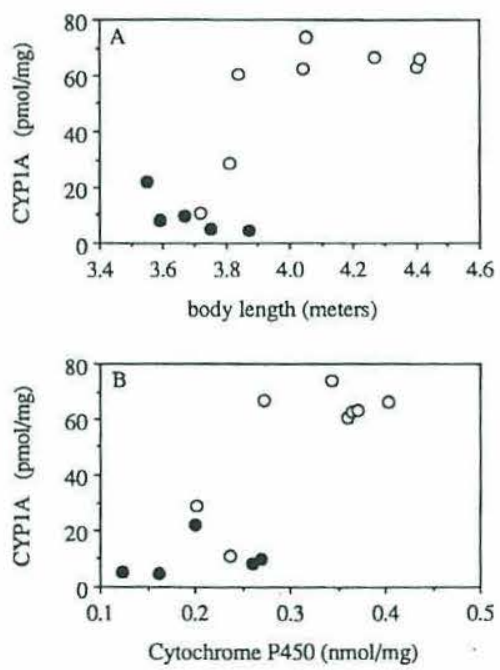

FIG. 5. Correlations of CYPIA content to length and to native cytochrome P450. (A) Hcpatic microsomal CYP1A, measured with MAb 1-12-3 and expressed as pmol P450E equivalents/mg, versus beluga body length. (B) Hepatic microsomal CYPIA versus microsomal cytochrome P450, measured spectrophotometrically as in Fig. 1. Closed circles, females; open circles, males. 
the relationship of CYPIA to native P450 content (Fig. 5B). The individuals fell into two clusters: larger males had consistently higher hepatic content of CYP1A and P450, while content in the females was substantially lower. Interestingly, the two smallest males had CYPIA and P450 contents that were more like those in females. All individuals in this study were most likely sexually mature adults at the time of capture, although the smaller males may have been young adults, according to growth curves for beluga from the MacKenzie Delta (Stewart and Stewart, 1989; Doidge, 1990).

Immunodetectable CYP1A accounts for $16.5 \%( \pm 5.8 \%)$ of native $\mathrm{P} 450$ in male samples, and $4.9 \%( \pm 3.4 \%)$ of native P450 in female samples, assuming equal reactivity of beluga and scup CYP1A forms with the antibody. However, because antibody reactivity may vary among species, the values obtained for beluga CYPIA represent relative rather than absolute amounts of $\mathrm{P} 450 \mathrm{E}$ homologue.

Cytochrome P4501A 1 is generally the primary catalyst of EROD and $\mathrm{AHH}$ activities in mammals and fish. Consistent with this, strong positive correlations were found between rates of EROD and $\mathrm{AHH}$, and the content of CYP1A in beluga. Relationships between EROD and CYP1A were characterized by $r^{2}=0.932$; between AHH and CYP1A by $r^{2}=0.853$; between EROD and AHH by $r^{2}=0.853$ (Fig. 6). Interestingly, there was also a strong positive correlation between these parameters and PROD activity (PROD and CYP1A, $r^{2}=0.945$; PROD and EROD, $r^{2}=0.912$ ) (Fig. 6). Results for one individual were excluded from the analysis of CYPIA content versus catalytic activity: a male with high (male-typical) levels of CYP1A and total P450, but with catalytic activities among the lowest of all the samples (Fig. 6). This is inexplicable in terms of sample degradation, as indicated by the high spectral P450 level and average P450/P420 ratio for this sample. Low rates of P450 activities in this individual might be attributable to low NADPH cytochrome c (P450) reductase activity. Reductase activity in this sample was the second lowest of 13 measured (67 $\mathrm{nmol} / \mathrm{min} / \mathrm{mg}$ ).

No correlation was found between beluga CYPIA content and $\mathrm{E}_{2}-2 \mathrm{OH}$ ase activity, nor between $\mathrm{E}_{2}-2 \mathrm{OH}$ ase and the other activities.

\section{Immunodetection of Other P450 Isozymes}

Western blot analysis was conducted to determine whether beluga whales express proteins related to CYP2B and CYP2E. For each immunoblot, four samples were chosen: two with relatively high CYP1A content and activity, and two with relatively low CYPIA content and activity.

The samples were blotted with three antibodies to members of the phenobarbital-inducible mammalian CYP2B subfamily, and one antibody to an apparent fish CYP2B, scup P450B. (Scup P450B and rat CYP2B1/2 show strong and exclusive reciprocal cross-reactivity.) The


FIG. 6. Correlations between monooxygenase activity and immunodetectable CYPIA in beluga liver microsomes. (A) EROD vs CYPIA. (B) AHH vs CYPIA. (C) PROD vs CYP1A. Closed circles are female and open circles are male beluga. CYP1A was quantified by immunodetection with MAb 1-12-3 and is expressed as pmol P450E equivalents/mg. Enzyme assays were conducted as described under Materials and Methods. Results with the one male that had high (male-typical) levels of CYPIA and exceptionally low catalytic activities (see Results) were not included in the regression analysis.

polyclonal anti-scup P450B (Fig. 7A) showed no reaction with beluga microsomes in the molecular weight region of 46- to $52-\mathrm{kDa}$, where scup and rat CYP2B forms migrate. In all seven lanes of this blot, staining is apparent in the high-molecular-weight region; this is an artifact which is commonly seen when using these polyclonal antibodies, and it is concluded to be nonspecific. The beluga samples each have a more discrete band just below this nonspecific staining area, in the region of approximately $60-$ to $70-\mathrm{kDa}$. The identity of this protein is not known and will require further investigation. Beluga microsomes did not crossreact with either the polyclonal (Fig. 7B) or the monoclonal (Fig. 7C) antibodies against rat CYP2B1. However, a polyclonal antibody to rabbit CYP2B4 (Fig. 7C) did recognize a distinct protein in each of the four beluga samples examined. This band had an electrophoretic migration near that of rat CYP2B1. Polyclonal anti-dog CYP2B11 recognized at least two bands in beluga liver microsomes with migration similar to that of authentic dog 2B11 (not shown). Thus, beluga appear to express a P450 form with some im- 
A
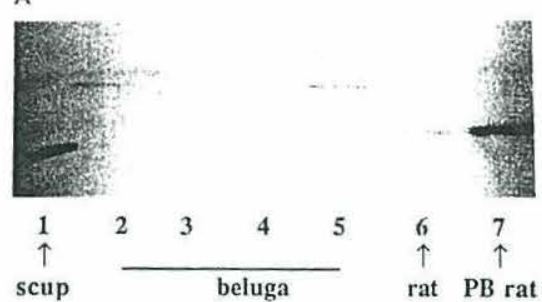

B

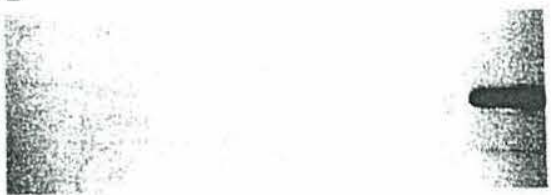



C
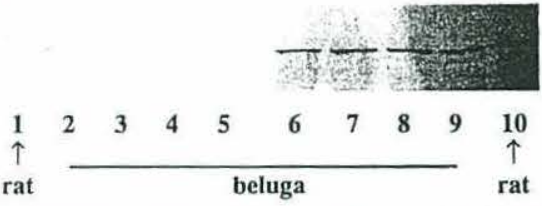

FIG. 7. SDS-PAGE immunoblots of beluga liver microsomes with antibodies against CYP2B forms. Beluga microsomal protein loading was 100 $\mu \mathrm{g} / \mathrm{lanc}$ in $\mathrm{A}$ and $\mathrm{B}$ on a large gel, and $60 \mu \mathrm{g} / \mathrm{lane}$ in $\mathrm{C}$ on a mini-gel. (A) Anti-P450B, a polyclonal Ab raised against scup P450B, a likely CYP2B form: lane I, untreated scup liver microsomes ( $18 \mu \mathrm{g})$; lanes 2,3 , and 5 were male and lane 4 a female beluga; lane 6 , untreated rat liver microsomes ( $30 \mu \mathrm{g})$; lane 7, phenobarbital-treated rat liver microsomes $(30 \mu \mathrm{g})$. (B) Anti-P450PB4, a polyclonal Ab raised against rat CYP2B1: lane 1, untreated scup liver microsomes (18 $\mu \mathrm{g})$; lanes 2, 3, and 5 were male and lane 4 a female beluga; lane 6 , untreated rat liver microsomes $(30 \mu \mathrm{g})$; lane 7 , phenobarbital-treated rat liver microsomes $(30 \mu \mathrm{g})$. (C) MAb 4-29-5p4, raised against rat CYP2B1 (lanes 1-5), and anti-P450LM2, a polyclonal Ab raised against rabbit CYP2B4 (lancs 6-10): lancs I and 10, untreated rat liver microsomes (60 $\mu \mathrm{g})$; lanes 2, 3, 8, and 9 were male and lanes 4, 5, 6, and 7 were female beluga Molecular weights of rat CYP2B1 and scup P450B (apparent 2B) are 52 and $46 \mathrm{kDa}$, respectively.

munochemical similarity to CYP2B forms other than CYP2B1/2, but the degree of structural similarity with these other form is unknown.

The samples were blotted with two polyclonal antibodies raised against the alcohol and ketone-inducible rat CYP2E1. One of these antibodies detected a single crossreacting band in the $\mathrm{P} 450$ region for each sample, while the second antibody recognized two distinct bands in each sample (Fig. 8). This indicates the possible expression in beluga of P450 forms related to rat CYP2E1.

\section{In Vitro Metaholism of Benzo[a]pyrene}

Benzo $[a]$ pyrene is a model polycyclic aromatic hydrocarbon which is known to be oxygenated at several different

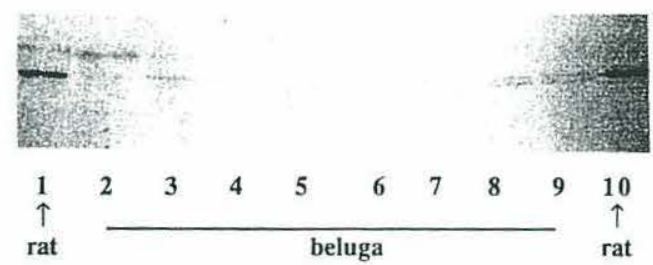

FIG. 8. SDS-PAGE immunoblot of beluga liver microsomes with polyclonal anti-rat CYP2E1. Protein loading was $60 \mu \mathrm{g} /$ lanc on a mini-gel. Lanes 1-5, anti-RLM6 (gift of J. Schenkman); lanes 6-10, anti-P4502E1 (from Oxygene, Dallas). Lanes 1 and 10 are untreated rat liver microsomes ( $60 \mu$ g protein); lanes $2,3,8$, and 9 are male beluga; lanes $4,5,6$, and 7 are female beluga. positions by mammalian P450 enzymes (Wiebel et al., 1975; Pezzuto et al., 1978). In vitro BP metabolites formed by beluga liver microsomes and analyzed by HPLC showed a typical suite of dihydrodiol, quinone, and hydroxy derivatives (Fig. 9). Amounts of the metabolites detected are reported in Table 3, along with percentages of total recovered metabolites. In both male and female samples, dihydrodiols made up the majority of the metabolites (approximately $50 \%$ ), with the rest composed of mono-hydroxy compounds $(23-24 \%)$ and quinones $(24-25 \%)$. The preference

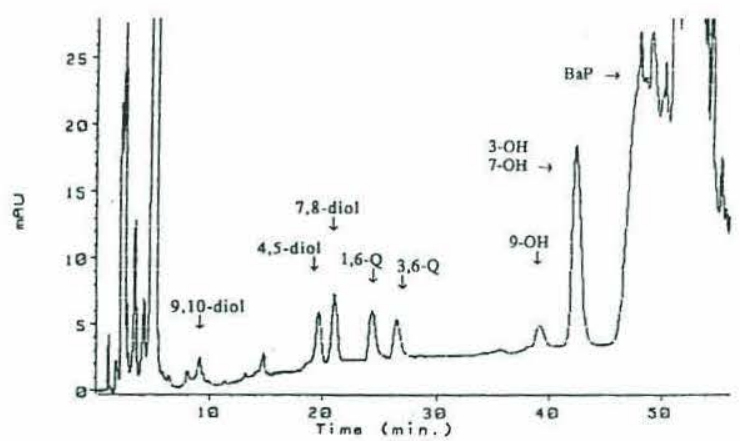

FIG. 9. Benzo[a]pyrene metabolite profile from a male beluga. Hepatic microsomes were incubated with $\left[{ }^{3} \mathrm{H}\right] \mathrm{BP}$ for $20 \mathrm{~min}$ and metabolites were detected by HPLC, as described under Materials and Methods. Metabolite identities are indicated in the figure and in Table 3. 
WHITE ET AL.

TABLE 3

Profile of Benzo[a]pyrene Metabolites Produced by Liver Microsomes of Beluga Whale

\begin{tabular}{lcc}
\hline \multicolumn{1}{c}{ Metabolite $^{a}$} & Female $(n=3)(\%)^{b}$ & Male $(n=3)(\%)^{b}$ \\
\hline 9.10-Dihydrodiol & $16.6 \pm 6.6^{b}$ & $21.6 \pm 3.2$ \\
4,5-Dihydrodiol & $24.4 \pm 3.6$ & $22.3 \pm 4.4$ \\
7,8-Dihydrodiol & $9.5 \pm 8.3$ & $9.3 \pm 1.8$ \\
1,6-Quinone & $14.9 \pm 1.2$ & $13.0 \pm 2.2$ \\
3,6-Quinone & $10.5 \pm 4.4$ & $10.9 \pm 4.3$ \\
9-OH & $8.8 \pm 2.4$ & $5.9 \pm 1.4$ \\
3-OH and 7-OH & $15.3 \pm 4.7$ & $17.1 \pm 7.4$ \\
BP metabolized & & \\
(\% of substrate) & $3.0 \pm 1.7$ & $7.9 \pm 2.8$ \\
(pmol/min/mg) & $16.6 \pm 9.9$ & $83.5 \pm 24.9$ \\
\hline
\end{tabular}

Metabolites prepared in $20 \mathrm{~min}$ incubations, extracted, and eluted from an ODS reverse-phase column as described under Materials and Methods, and identified by elution time and by uv-spectra compared to standards.

${ }^{b}$ All values are listed as percentages of total recovered metabolites, in order of elution from the column.

for hydroxylation at the various positions did not differ between the sexes: the dominant metabolites in both were the 4,5-dihydrodiol and the 9,10-dihydrodiol. An additional metabolite, present at a very low concentration, was detected in some samples. This compound eluted after the 3,6-quinone and before the 9-hydroxy, which is the region where the 6,12-quinone, the 4,5-epoxide, and the 6-hydroxymethyl metabolites elute. However, the small quantity of the unknown compound precluded identification from its absorption spectrum.

\section{DISCUSSION}

Marine mammals are exposed to pollutant chemicals throughout the world oceans, yet there have been few detailed studies of the effects of such exposure. Various cytochrome $\mathrm{P} 450$ forms are induced by exposure to organic pollutants and function in the primary metabolism of these compounds. Characterization of cetacean P450 systems is necessary to fully evaluate the capacity of these animals to dispose of foreign compounds, and should provide insight into the mechanisms of xenobiotic impact on whale populations, information that cannot be obtained experimentally in these marine mammals. Moreover, CYP1A induction is increasingly recognized as a valid biomarker of exposure of animals and humans to foreign chemicals that are Ah-receptor agonists (Stegeman et al., 1992). Recent studies have disclosed an apparent Ah-receptor in liver of beluga (Hahn et al., 1992, 1994), suggesting that beluga have a mechanism for response to these compounds similar to that in other mammals. In this study we describe the characteristics of hepatic microsomal enzymes in beluga, including a strong induction of a CYP1A (possibly a CYP1A1) in the liver of these animals, correlated with the content of PCBs in the blubber (see below).

\section{Monooxygenase Systems}

Microsomal monooxygenase enzymes have been analyzed in a few other cetacean species, and those results are comparable to the data reported here (Table 4). The levels of native cytochrome P450, cytochrome b5, NADPH cytochrome c (P450) reductase activity, EROD activity, and $\mathrm{AHH}$ activity in this study were very similar to those found in minke whale from Arctic waters (Goksøyr et al., 1986, 1988). Like beluga, minke whale microsomes had appreciable levels of MAb 1-12-3 cross-reactive protein, and no cross-reaction with antibodies raised against rat CYP2B. Total P450 content in beluga is close to the P450 contents measured in three other cetacean species, short-finned pilot whale, striped dolphin, and killer whale, caught off the coast of Japan (Watanabe et al., 1989). EROD activity levels measured in the four species fall within an overlapping range.

Based on statistical correlations (Fig. 6), we conclude that a beluga CYP1A is the primary catalyst for EROD and $\mathrm{AHH}$ activities, as in most vertebrates that have been examined. Interestingly, rates of PROD activity - which were relatively low in these animals-were also strongly correlated with CYP1A content. In rat liver, PROD activity is catalyzed primarily but not exclusively by PB-inducible CYP2B forms (Burke et al., 1985). PROD activity has also been found to be induced by hydrocarbon-type inducers in rat and mouse (Burke et al., 1985; Kelley et al., 1990). Our results suggest that the PROD activity in these beluga liver microsomes is a CYP1A activity.

Estradiol 2-hydroxylase activity was assayed in part to determine whether sample degradation might interfere with interpretations of the data for the $\mathrm{MO}$ activities we measured. If the positive correlations found among EROD, $\mathrm{AHH}, \mathrm{PROD}$, and CYP1A were attributable to differential degrees of P450 degradation, rather than to differences in the catalytic capacities of the individual samples, it would be expected that $\mathrm{E}_{2}-2 \mathrm{OH}$ ase activity would show a similar pattern of positive correlation. As no such correlation was found, it can be concluded that (1) sample degradation was not responsible for the observed correlations between isozyme content and catalytic activities, and (2) $\mathrm{E}_{2}-2 \mathrm{OH}$ ase activity is not catalyzed by CYPIA in beluga whales. $\mathrm{E}_{2}-2 \mathrm{OHase}$ is catalyzed largely by CYP1A2 in rat (Dannan et al., 1986). When beluga microsomes were blotted with an anti-mouse CYP1A1 polyclonal antibody which recognizes both CYP1A1 and CYP1A2 in mice and rats, there was no evidence of an electrophoretically distinct beluga protein immunologically related to CYP1A2 (Fig. 4). Microsomal $\mathrm{E}_{2}-2 \mathrm{OHase}$ activity is also catalyzed to varying degrees by 
P450 IN BELUGA WHALE

TABLE 4

Comparison of Liver Microsomal Monooxygenase Components and Activities in Different Species of Adult Cetacean

\begin{tabular}{lcccc}
\hline & $\begin{array}{c}\text { Beluga whale } \\
(13)\end{array}$ & $\begin{array}{c}\text { Minke whale } \\
(10)^{a}\end{array}$ & $\begin{array}{c}\text { Pilot whale } \\
(33)^{b}\end{array}$ & $\begin{array}{c}\text { Striped dolphin } \\
(5)^{b}\end{array}$ \\
\hline cyt P450 & $0.274 \pm 0.089$ & $0.35 \pm 0.09$ & $0.171 \pm 0.050$ & $0.186 \pm 0.021$ \\
cyt b5 $^{c}$ & $0.222 \pm 0.034$ & $0.25 \pm 0.03$ & n.m. & n.m. \\
Reductase $^{d}$ & $77 \pm 8$ & $76 \pm 24$ & $64 \pm 13$ & $93 \pm 18$ \\
EROD $^{c}$ & $291 \pm 261$ & $740 \pm 170$ & $42 \pm 42$ & $191 \pm 0.068$ \\
AHH $^{c}$ & $191 \pm 156$ & $220 \pm 60$ & $7.8 \pm 4.2$ & $35 \pm 15$ \\
\hline
\end{tabular}

Note. Values are means \pm SD. Numbers in parentheses are sample sizc. n.m., parameter not measured.

a Data reported by Goksoyr et al., 1988.

${ }^{b}$ Data reported by Watanabe et al., 1989.

' $\mathrm{nmol} / \mathrm{mg}$.

${ }^{d} \mathrm{nmol} / \mathrm{min} / \mathrm{mg}$.

c $\mathrm{pmol} / \mathrm{min} / \mathrm{mg}$.

several other P450 forms in rat (Dannan et al., 1986). Thus, the $\mathrm{E}_{2}-2 \mathrm{OH}$ ase catalyst in beluga liver is presently unknown.

Benzo $[a]$ pyrene, a prevalent environmental pollutant and a model P450 substrate, is metabolized by beluga liver microsomes to a suite of oxygenated products including benzo-ring and K-region dihydrodiols (DHD), mono-hydroxy compounds, and quinones. CYPI A isoforms are generally most active in benzo-ring metabolism of BP, including the formation of the proximal carcinogen BP-7,8-dihydrodiol. This metabolite can be converted to the highly carcinogenic (+)-(7R,8S)-diol-(9S,10R)-epoxide. The suite of metabolites formed by beluga liver microsomes is similar to that produced by liver microsomes of rat (Pezzuto et al., 1978; Wroblewski et al., 1988). Based on studies of CYP1A1 forms stably expressed in Chinese hamster V79 cells, the major BP-DHD produced by rat CYP1Al are 9,10- and 7,8-DHD as well as the K-region (4,5-) DHD. By contrast, CYPIAI from humans is more specific for metabolism on the benzo-ring of BP, and does not form the 4,5DHD (J. Doehmer, personal communication). Fish CYPIA proteins also metabolize $\mathrm{BP}$ predominantly to benzo-ring DHD and form little or no K-region DHD (Stegeman et al., 1984). Analysis of CYP1A from beluga may shed light on the structural features associated with regioselectivity for BP metabolism.

\section{Environmental Induction of CYP1A}

Concentrations of organic chemical pollutants in the Canadian Arctic are in general quite low, and there are no industrial or population centers, and thus limited local sources of contamination. However, the Arctic is not free from anthropogenic pollutants. Polycyclic aromatic hydrocarbons were found in MacKenzie Delta sediments, and DNA adducts, ostensibly of PAH, were detected in the livers of beluga from the MacKenzie Delta (Ray et al.,
1991). PCBs and other organochlorines were detected in tissues of beluga, ringed seal, polar bear, and narwhal from the Canadian Arctic (Norstrom et al., 1990; Muir et al., 1992).

Blubber samples from the same beluga described in this study were analyzed for content of individual PCB congeners (Metner $e t$ al., in preparation). Among the congeners analyzed were nine (IUPAC numbers $37,81,77$, $126,169,105,114,118,156)$ which are known to be toxic and/or to strongly induce CYP1A forms in rats (Goldstein and Safe, 1989). These are trichloro- to hexachlorobiphenyls, each possessing two para substituents and either one or zero ortho substituents. Among the 13 animals examined, we found a strong positive correlation between PCB content in blubber (expressed as the sum of the nine congeners) and CYP1A enzyme content in the liver (Fig. 10; $r^{2}=$ 0.875 ). The strong correlation between the content of known CYP1A inducers and the content of CYP1A in liver indicates that the CYP1A in beluga was induced by these compounds, or by compounds that often covary with PCBs in the environment, such as polychlorinated dibenzo- $p$ dioxins or polychlorinated dibenzofurans. It is possible that PCB stores in fatty tissues were mobilized during a period of starvation, just prior to capture, contributing to enzyme induction.

Cytochrome P450 content, relative CYP1A content, and all catalytic activities assayed were significantly greater in male beluga than in female. Sex differences in monooxygenase activities seen in rat have been linked to hormonal regulation of different P450 isoforms (Waxman et al., 1985; Dannan et al., 1986). In fish, CYP1A1 has been found to be suppressed in reproductively active females by the hormone estradiol (Gray et al., 1991). However, several studies have shown that CYP1A1 is not sexually differentiated in rats, either untreated (Waxman et al., 1985; Cresteil et al., 1986 ) or treated with CYP1A1 inducers (Warren and Bellward, 1978: Waxman et al.. 1985). 
WHITE ET AL.

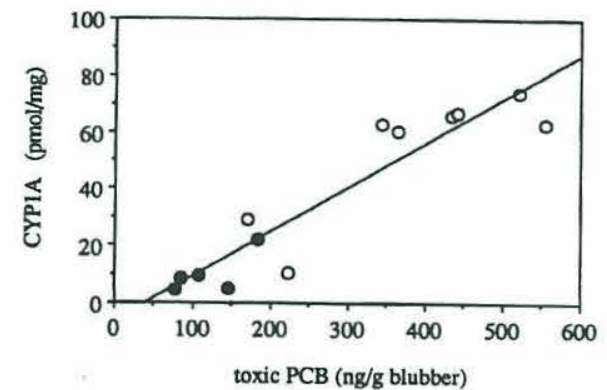

FIG. 10. CYPIA content in beluga liver microsomes versus concentration of non-ortho and mono-ortho PCBs in blubber samples of the same animals. CYPIA was quantified as in Fig. 3. The content of PCB congeners (referred to as "toxic" PCBs) was determined by solvent-extraction of blubber and analysis by gas chromatography, as described by Muir $e t$ al. (1990). Content is expressed as the sum of nine congeners: IUPAC numbers $37,81,77,126,169,105,114,118,156$. The $r^{2}$ was 0.875 . Closed circles, females; open circles, males.

A further explanation for the sex difference in CYP1A content in the beluga may involve enzyme induction. Those animals with the highest levels of hepatic CYP1A (six males) also had the greatest blubber concentrations of non-ortho and mono-ortho PCBs, whereas those animals with lower CYP1A content and related activities (five females, two small males) had much lower PCB content in body fat (Fig. 10). There are numerous reports in the literature of adult male cetaceans having higher blubber concentrations of organochlorines than adult females (Martineau et al., 1987; Aguilar and Borrell, 1988; Subramanian et al., 1988; Muir et al., 1990). Several hypotheses might explain the sex difference in PCB content. The lower concentrations found in females may be the result of past lactation and/or pregnancy, both means by which organochlorines can be eliminated from female cetaceans (Duinker and Hillebrand, 1979; Subramanian et al., 1988). Additionally, it has been suggested that differences in diet between male and female beluga may contribute to differences in contaminant loads (Martineau et al., 1987). A third explanation may be that the larger males are the older members of the group, and thus would have had an increased time over which to accumulate persistent toxicants. Consistent with this latter hypothesis, PCB content in the blubber of Saint Lawrence beluga was found to increase exponentially with increasing age (Martineau et al., 1987).

\section{Cytochrome $P 4502 B$}

Samples in this study failed to show cross-reactivity with polyclonal antibodies raised against rat CYP2B1. Similarly, antibodies against a teleost fish enzyme that is structurally related to CYP2B1 (scup P450B) (Stegeman et al., 1990) failed to recognize a protein in beluga liver microsomes with a molecular weight near that of P450. This evidence indicating the lack of expression of a protein related to rat CYP2B1/2B2 in beluga is similar to results obtained with another cetacean. Antibodies raised against rat CYP2B failed to cross-react with minke whale liver microsomes (Goksøyr et al., 1988). The absence of a signal on immunoblots may be due either to the lack of a gene encoding a protein homologous to CYP2B forms or to low/no expression of such a gene in beluga liver. Interestingly, $\mathrm{PCB}$ congeners known to be inducers of CYP2B in mammals were present in these beluga (Metner et al., in preparation).

Catalytic data seem to support the immunochemical data indicating little CYP2B-like activity in cetaceans. While CYP2B1 is the primary catalyst of PROD activity in rat liver microsomes (Burke et al., 1985), PROD activity in these beluga was very slight and was strongly correlated with immunodetectable CYP1A (Fig. 6), suggesting that the PROD activity detected here is catalyzed primarily by CYP1A. Similarly, aldrin epoxidase activity was found to be very low in short-finned pilot whale, striped dolphin, and killer whale, compared to that in rat (Watanabe et al., 1989); this activity is catalyzed largely by PB-inducible CYP2B forms in rat (Wolff and Guengerich, 1987). The in vitro production of a $\mathrm{K}$-region $\mathrm{BP}$ metabolite (4,5-dihydrodiol) by beluga (Fig. 9) could reflect CYP2B-like activity, but in rats this metabolite appears to be formed by CYP1A as well as by CYP2B forms (e.g., Pezzuto et al., 1978).

PCB residue data from cetaceans also suggest that these animals have little CYP2B activity. Tanabe et al. (1988) found selective retention of meta-para unsubstituted PCB congeners in the tissues of Dall's porpoise and striped dolphin, relative to congener concentrations in their food organisms. Preferential retention of these PCBs, which are believed to be metabolized primarily by CYP2B forms, suggests the lack of CYP2B activity in these cetaceans. Likewise, Norstrom et al. (1992) found a higher relative abundance of meta-para unsubstituted PCBs in arctic beluga than in charr, polar bear, walrus, and ringed seal taken from the same habitat, suggesting a distinct inability of cetaceans to metabolize these congeners. Consistent with this, we have found that beluga liver microsomes have a very low capacity for metabolism of $2,2^{\prime}, 5,5^{\prime}-\mathrm{TCB}$ compared to that of control rat liver microsomes (White and Stegeman, in preparation).

Given the above, the presence of protein bands crossreacting with anti-rabbit CYP2B4 or anti-dog CYP2B11 is intriguing. CYP2B4 is the major PB-inducible P450 form in rabbit liver, and its protein sequence is $77 \%$ identical to that of rat CYP2B1, as deduced from cDNA sequences (Tarr et al., 1983; Komori et al., 1988). The question of whether CYP2B genes are present and expressed in cetaceans will be resolved with further catalytic and immunochemical studies, including inhibition assays with polyclonal antibodies against CYP2B, and a search for CYP2B 
genes. It will be interesting in this regard to compare cetaceans with some of their terrestrial relatives among the ungulates, the line from which cetaceans diverged 50-60 million years ago. Such comparisons of the carnivorous Odontoceti with the herbivorous Bovidae might be particularly interesting, possibly shedding light on the role of plant natural products in diversification within the CYP2B subfamily.

As with CYP2B, interesting questions concern other $\mathrm{P} 450$ forms in the cetacea. The detection of proteins reacting with antibodies to CYP2EI suggests the expression of homologues to the ketone inducible rat form. Rat CYP2E1 is induced in starvation; thus, the suspected starvation of these beluga may have contributed to the appearance of CYP2E-like proteins.

In summary, this study documents the expression and activity of cytochrome P450 enzymes in the liver of a marine mammal species, the beluga whale, thought to be at risk of pollution effects in some regions. CYPIA proteins have been implicated in organic pollutant metabolism in many vertebrate species, and appear to serve a similar function in beluga whale. We conclude that the CYP1A content most likely reflects xenobiotic ligand binding to the Ah-receptor, which suggests that toxicity could be mediated by the receptor in these animals. The relationship between CYP1A content and PCB content indicates that measurement of CYPIA is likely to be a useful biomarker for exposure of marine mammals to Ah-receptor agonists. No evidence was found in beluga for an enzyme immunochemically related to rat CYP2B1/2, possibly indicative of the divergent evolution of marine and terrestrial mammals. These results offer insight into the mechanisms associated with toxic effects in beluga, contribute to the framework for further study of the effects of xenobiotics on marine mammal populations, and may provide a paradigm for evaluating chemical risks in natural populations of mammals.

\section{ACKNOWLEDGMENTS}

We thank Lothar Dahlke (Department of Fisheries and Oceans, Winnipeg) for sample collection, Drs. Ncil Blough and Sarah Green (Woods Hole Oceanographic Institution) for assistance with fluorometry, Bruce Woodin (WHOI) for technical assistance, and Cheri Recchia (WHOI) for commenting on the manuscript. We are also grateful to those who provided antibodics used in this study. Contribution No. 8461 of the Woods Hole Oceanographic Institution.

\section{REFERENCES}

Addison, R., and Brodie, P. (1984). Characterization of ethoxyresorufin O-deethylase in grey seal Halichoerus grypus. Comp. Biochem. Physiol. C 79, 261-263.

Addison, R., Brodie, P., Edwards, A., and Sadler, M. (1986). Mixed function oxidase activity in the harbour seal (Phnca vitulina) from Sable Is., N.S. Comp. Biochem. Physiol. C' 85, 121-124.

Aguilar, A., and Borrell, A. (1988). Age- and sex-related changes in organo- chlorine compound levels in fin whales (Balaenopter physalus) from the eastern North Atlantic. Mar. Environ. Res. 25, 195-211.

Binder, R. L., and Stegeman, J. J. (1980). Induction of aryl hydrocarbon hydroxylase activity in embryos of an estuarine fish. Biochem. Pharma col. 29, 949-951.

Burke, M. D., Thompson, S., Elcombe, C. R., Halpert, J., Haaparanta, T. and Mayer, R. T. (1985). Ethoxy-, pentoxy-, and benzyloxyphenones and homologues: A series of substrates to distinguish between different induced cytochromes P-450. Biochem. Pharmacol. 34, 3337-3345

Cresteil, T., Beaune, P., Celier, C., Leroux, J. P., and Guengerich, F. P. (1986). Cytochrome P-450 isoenzyme content and monooxygenase activities in rat liver: Effect of ontogenesis and pretreatment by phenobarbital and 3-methylcholanthrene. J. Pharmacol. Exp. Ther. 236, 269-276.

Dannan, G. A., Porubck, D. J., Nelson, S. D., Waxman, D. J., and Guengerich, F. P. (1986). 17 $\beta$-cstradiol 2- and 4-hydroxylation catalyzed by rat hepatic cytochrome $\mathrm{P} 450$ : Roles of individual forms, inductive effects, developmental patterns, and altcrations by gonadectomy and hormone replacement. Endocrinology 118, 1952-1960.

Doidge, D. W. (1990). Age-length and length-weight comparisons in the beluga, Delphinapterus leucas. Can. Bull. Fish. Aquat. Sci. 224, 59-68.

Duignan, D. B., Sipes, I. G., Leonard, T. B., and Halpert, J. R. (1987). Purification and characterization of the dog hepatic cytochrome $P-450$ isozyme responsible for the metabolism of $2,2^{\prime}, 4,4^{\prime}, 5,5^{\prime}$-hexachlorobiphenyl. Arch. Biochem. Biophys. 255, 290-303.

Duinker, J. C., and Hillebrand, M. T. J. (1979). Mobilization of organochlorines from female lipid tissuc and transplacental transfer to fetus in a harbour porpoise (Phocoena phocoena) in a contaminated area. Bull. Environ. Contam. Toxicol. 23, 728-732.

Duinker, J. C., Hillebrand, M. T. J., Zeinstra, T., and Boon, J. P. (1989) Individual chlorinated biphenyls and pesticides in tissues of some cetacean species from the North Sea and the Atlantic Ocean; Tissue distribution and biotransformation. Aquat. Mamm. 15, 95-124.

Engelhardt, F. (1982). Hydrocarbon metabolism and cortisol balance in oil-exposed ringed seals, Phoca hispida. Comp. Biochem. Physiol. C 72, 133-136.

Favreau, L. V., Malchoff, D. M., Mole, J. E., and Schenkman, J. B. (1987). Responses to insulin by two forms of rat hepatic microsomal cytochrome P-450 that undergo major (RLM6) and minor (RLM5b) elevations in diabetcs. J. Biol. Chem. 262, 14319-14326.

Frey, A. B., Waxman, D. J., and Kreibich, F. (1985). The structure of phenobarbital-inducible rat liver cytochrome P-450 isoenzyme PB-4. Production and characterization of site-specific antibodies. J. Biol. Chem. 260, 15253-15265.

Goksøyr, A., Andersson, T., Forlin, L., Stenersen. J., Snowberger, E. A., Woodin, B. R., and Stegeman, J. J. (1988). Xenobiotic and steroid metabolism in adult and foetal piked (minke) whales, Balaenoptera acutorostrala. Mar. Environ. Res. 24, 9-13.

Goksøyr, A., Beyer, J., Larsen, H. E., Andersson, T., and Forlin, L. (1992). Cytochrome P450 in seals: monooxygenase activities, immunochemical cross-reactions and response to phenobarbital treatment. Mar. Environ. Res. 34, 113-116.

Goksøyr, A., Solbakken, J., Tarlebø, J., and Klungsøyr, J. (1986). Initial characterization of the hepatic microsomal cytochrome P-450-system of the piked whale (minke) Balaenoptera acutorostrata. Mar. Environ. Res $19,185-203$.

Goldstein, J. A., and Safe, S. (1989). Mechanism of action and structureactivity relationships for the chlorinated dibenzo- $p$-dioxins and related compounds. In Halogenated Biphenyls, Terphenyls, Naphthalenes, Dibenzodioxins and Related Products (R. D. Kimbrough, and A. A. Jensen, Eds.), pp. 239-293. Elsevier, Amsterdam.

Gray, E. S. (1988). Sexual patterns of monooxygenase function in the liver 
WHITE ET AL.

of marine teleosts and the regulation of activity by estradiol. Ph.D. Thesis, MIT/WHOI

Gray, E. S., Woodin, B. R., and Stegeman, J. J. (1991). Sex differences in hepatic monooxygenases in winter flounder (Pseudopleuronectes americanus) and scup (Stenotomus chrysops) and regulation of $\mathrm{P} 450$ forms by estradiol. J. Exp. Zool. 259, 330-342.

Hahn, M. E., Poland, A., Glover, E., and Stegeman, J. J. (1992). The Ah receptor in marine animals: Phylogenetic distribution and relationship to P450IA inducibility. Mar. Environ. Res. 34, 87-92.

Hahn, M. E.. Poland, A., Glover, E., and Stegeman, J. J. (1994). Photoaffinity labeling of the Ah receptor: Phylogenetic survey of divcrse vertebrate and invertebrate species. Arch. Biochem. Biophys. 310, 218-228.

Hellou, J., Upshall, C., Ni, I. H., Payne, J. F., and Huang, Y. S. (1991). Polycyclic aromatic hydrocarbons in harp seals (Phoca groenlandica) from the northwest Atlantic. Arch. Environ. Contam. Toxicol. 21, 135140.

Johannesen, K. A. M., and DePierre, J. W. (1978). Measurement of cytochrome $\mathrm{P} 450$ in the presence of large amounts of contaminating hemoglobin and methemoglobin. Anal. Biochem. 86, 725-732.

Kelley, M., Womach, J., and Safe, S. (1990). Effects of cytochrome P-450 monooxygenase inducers on mouse hepatic microsomal metabolism of testosterone and alkoxyresorufins. Biochem. Pharmacol. 39, 19911998.

Kloepper-Sams, P. J., Park, S. S., Gelboin, H. V., and Stegeman, J. J (1987). Specificity and cross-reactivity of monoclonal and polyclonal antibodies against cytochrome P450E of the marine fish scup. Arch. Biochem. Biophys. 253, 268-278.

Klotz, A. V., Stegeman, J. J., and Walsh, C. (1984). An alternative 7-cthoxyresorufin O-deethylase activity assay: A continuous visible spectrophotometric method for measurement of cytochrome P-450 monoxygenase activity. Anal. Biochem. 140, 138-145.

Komori, M., Imai, Y., Tsunasawa, S., and Sato, R. (1988). Microheterogeneity in the major phenobarbital-inducible forms of rabbit liver microsomal cytochrome $\mathrm{P}-450$ as revealed by nucleotide sequencing of cloned cDNAs. Biochemistry 27, 73-80.

Martineau, D., Beland, P., Desjardins, C., and Lagace, A. (1987). Levels of organochlorine chemicals in tissues of beluga whales (Delphinapterus leucas) from the St. Lawrence Estuary, Quebec, Canada. Arch. Environ. Contam. Toxicol. 16, 137-147.

Martineau, D., Lagace, A., Beland, P., Higgins, R., Armstrong, D., and Shugart, L. R. (1988). Pathology of stranded beluga whales (Delphinapterus leucas) from the St. Lawrence Estuary, Quebec, Canada. J. Comp. Pathol. 98, 287-311.

Masse, R., Martineau, D., Tremblay, L., and Beland, P. (1986). Concentrations and chromatographic profile of DDT metabolites and polychlorobiphenyl (PCB) residues in stranded beluga whales (Delphinapterus leucas) from the St. Lawrence Estuary, Canada. Arch. Environ. Contam. Toxicol. 15, 567-579.

Muir, D. C., Ford, C. A., Stewart, R. E. A., Smith, T. G., Addison, R. F. Zinck, M. E., and Beland, P. (1990). Organochlorine contaminants in belugas, Delphinapterus leucas, from Canadian waters. Can. Bull. Fish. Aquat. Sci. 224, 165-190.

Muir, D. C. G., Ford, C. A., Grift, N. P., Stewart, R. E. A., and Bidleman, T. F. (1992). Organochlorine contaminants in narwhal (Monodon mon oceros) from the Canadian Arctic. Environ. Poll. 75, 307-316.

Muir, D. C. G., Wagemann, R., Grift, N. P., Norstrom, R. J., Simon, M., and Lien, J. (1988). Organochlorine chemical and heavy metal contaminants in white-beaked dolphins (Lagenorhynchus albirostris) and pilot whales (Globicephala melaena) from the coast of Newfoundland, Canada. Arch. Ezviron. Contam. Toxicol. 17, 613-629.
Nebert, D. W., and Gonzalez, F. J. (1987). P450 genes: Structure, evolution, and regulation. Annu. Rev. Biochem. 56, 945-993.

Nelson, D. R., Kamataki, T., Waxman, D. J., Guengerich, F. P., Estabrook, R. W., Feyereisen, R., Gonzalez, F. J., Coon, M. J., Gunsalus, I. C., Gotoh, O., Okuda, K., and Nebert, D. W. (1993). The P450 Superfamily-Update on new sequences, gene mapping, accession numbers, early trivial names of enzymes, and nomenclature. DNA Cell Biol. 12, $1-51$.

Norstrom, R. J., Muir, D. C. G., Ford, C. A., Simon, M., Macdonald, C. R., and Beland, P. (1992). Indications of P450 monooxygenase activities in beluga (Delphinapterus leucas) and narwhal (Monodon monoceros) from patterns of PCB, PCDD and PCDF accumulation. Mar Environ. Res. 34, 267-272.

Norstrom, R. J., Simon, M., and Muir, D. C. G. (1990). Polychlorinated dibenzo-p-dioxins and dibenzofurans in marine mammals in the Canadian north. Environ. Poll. 66, 1-19.

Omura, T., and Sato, R. (1964). The carbon monoxide-binding pigment of liver microsomes. J. Biol. Chem. 239, 2370-2378.

Ono, M., Kannan, N., Wakimoto, T., and Tatsukawa, R. (1987). Dibenzofurans a greater global pollutant then dioxins? Evidence from analyses of open ocean killer whale. Mar. Poll. Bull. 18, 640-643.

Park, S. S., Fujino, T., Miller, H., Guengerich, F. P., and Gelboin, H. V. (1984). Monoclonal antibodies to phenobarbital-induced rat liver cytochrome P-450. Biochem. Pharmacol. 33, 2071-2081.

Park, S. S., Miller, H., Klotz, A. V., Kloepper-Sams, P. J., Stegeman, J. J., and Gelboin, H. V. (1986). Monoclonal antibodies to liver microsomal cytochrome P-450E of the marine fish Stenotomus chrysops (scup): Cross-reactivity with 3-methylcholanthrene induced rat cytochrome P-450. Arch. Biochem. Biophys. 249, 339-350.

Pezzuto, J. M., Yang, C. S., Yang, S. K., McCourt, D. W., and Gelboin, H. V. (1978). Metabolism of benzo[a]pyrene and (-)-trans-7,8dihydroxy-7,8-dihydrobenzo[a]pyrene by rat liver nuclei and microsomes. Cancer Res. 38, 1241-1245.

Ray, S., Dunn, B. P., Payne, J. F., Fancey, L., Helbig, R., and Beland, P. (1991). Aromatic DNA-carcinogen adducts in beluga whales from the Canadian arctic and Gulf of St. Lawrence. Mar. Poll. Bull. 22, 392-396.

Reijnders, P. J. H. (1986). Reproductive failure in common seals feeding on fish from polluted coastal waters. Nature 324, 456-457.

Smith, P. K., Krohn, R. I., Hermanson, G. T., Mallia, A. K., Gartner, F. H., Provenzano, M. D., Fujimoto, E. K., Goeke, N. M., Olson, B. J., and Klenk, D. C. (1985). Measurement of protein using bicinchoninic acid. Anal. Biochem. 150, 76-85.

Snowberger, E. A., and Stegeman, J. J. (1987). Patterns and regulation of estradiol metabolism by hepatic microsomes from two species of marine teleosts. Gen. Comp. Endocrinol. 66, 256-265.

Stegeman, J. J., and Binder, R. L. (1979). High benzo[a]pyrene hydroxylase activity in the marine fish Stenotomus versicolor. Biochem. Pharmacol. 28, 1686-1688.

Stegeman, J. J., Binder, R. L., and Orren, A. (1979). Hepatic and extrahepatic microsomal electron transport components and mixed-function oxygenases in the marine fish Stenotomus versicolor. Biochem. Pharmacol. 28, 3431-3439.

Stegeman, J. J., Brouwer, M., DiGiulio, R. T., Forlin, L., Fowler, B. M., Sanders, B. M., and Van Veld, P. (1992). Molecular responses to environmental contamination: Enzyme and protein systems as indicators of contaminant exposure and effect. In Biomarkers. Biochemical, Physiological, and Histological Markers of Anthropogenic Stress (R. J. Huggett et al., Eds.), pp. 235-335. Lewis Publishers, Boca Raton, FL.

Stegeman, J. J., Smolowitz, R. M., and Hahn, M. E. (1991). Immunohistochemical localization of environmentally induced cytochrome P450IA I 


\section{P450 IN BELUGA WHALE}

in multiple organs of the marine teleost Stenotomus chrysops (scup). Toxicol. Appl. Pharmacol. 110, 486-504.

Stegeman, J. J., Woodin, B. R., and Binder, R. L. (1984). Patterns of benzo[a]pyrene metabolism by varied species, organs, and developmental stages of fish. Natl. Cancer Inst. Monogr. 65, 371-377.

Stegeman, J. J., Woodin, B. R., and Smolowitz, R. M. (1990). Structure, function and regulation of cytochrome $\mathrm{P}-450$ forms in fish. Biochem. Soc. Trans. 18, 19-21.

Stewart, B. E., and Stewart, R. E. A. (1989). Delphinapterus leucas. Mamm. Spec. 336, 1-8.

Subramanian, A., Tanabe, S., and Tatsukawa, R. (1988). Use of organochlorines as chemical tracers in determining some reproductive parameters in Dalli-type Dall's porpoise Phocoenoides dalli. Mar. Env. Res. 25, 161-174.

Tanabe, S., Watanabe, S., Kan, H., and Tatsukawa, R. (1988). Capacity and mode of PCB metabolism in small cetaceans. Mar. Mam. Sci. 4, 103-124.

Tarr, G. E., Black, S. D., Fujita, V. S., and Coon, M. J. (1983). Complete amino acid sequence and predicted membrane topology of phenobarbital-induced cytochrome P-450 (isozyme 2) from rabbit liver microsomes. Proc. Natl. Acad. Sci. USA 80, 6552-6556.

Testa, B., and Jenner, P. (1981). Inhibitors of cytochrome P-450s and their mechanism of action. Drug Metab. Rev. 12, 1-117.

Warren, P. M., and Bellward, G. D. (1978). Induction of aryl hydrocarbon hydroxylase by 3-methylcholanthrene in liver, lung and kidney of gonadectomized and sham-operated wistar rats. Biochem. Pharmacol. 27, 2537-2541.

Watanabe, S., Shimada, T., Nakamura, S., Nishiyama, N., Yamashita, N., Tanabe, S., and Tatsukawa, R. (1989). Specific profile of liver microsomal cytochrome P-450 in dolphin and whales. Mar. Environ. Res. 27, $51-65$.

Waxman, D., Dannan, G. A., and Guengerich, F. P. (1985). Regulation of rat hepatic cytochrome P-450: Age-dependent expression, hormonal imprinting, and xenobiotic inducibility of sex-specific isoenzymes. Biochemistry 24, 4409-4417.

Wiebel, F. J., Selkirk, J. K., Gelboin, H. V., Haugen, D. A., van der Hoeven, T. A., and Coon, M. J. (1975). Position-specific oxygenation of benzo[a]pyrene by different forms of purified cytochrome P-450 from rabbit liver. Proc. Natl. Acad. Sci. USA 72, 3917-3920.

Wolff, T., and Guengerich, F. P. (1987). Rat liver cytochrome P-450 isozymes as catalysts of aldrin epoxidation in reconstituted monooxygenase systems and microsomes. Biochem. Pharmacol. 36, 2581-2588.

Woodley, T. H., Brown, M. W., Kraus, S. D., and Gaskin, D. E. (1991). Organochlorine levels in North Atlantic right whale (Eubalaena glacialis) blubber. Arch. Environ. Contam. Toxicol. 21, 141-145.

Wroblewski, V. J., Gessner, T., and Olson, J. R. (1988). Qualitative and quantitative differences in the induction and inhibition of hepatic benzo[a]pyrene metabolism in the rat and hamster. Biochem. Pharmacol. 37, 1509-1517. 
CHAPTER EIGHT

Summary and Conclusions 


\section{Teleost studies}

3,3',4,4'-TCB is known to be a potent toxicant, CYP1A1 inducer, and CYP1A1 substrate in laboratory mammals. However, there have been many gaps in the information regarding TCB toxicity and TCB/CYP1A1 interactions in marine teleost species. Little has been known about the dose-response and time-course patterns of CYP1A1 induction in extrahepatic organs and cells of fish, about the histopathology elicited by TCB in target organs and cells of fish, and about the involvement of cytochromes P450 in TCB metabolism in fish. Although suppression of CYP1A1 protein content by planar PCB has been described or suggested in studies of fish, birds, and mammals, the specificity and mechanisms of this suppression have not been characterized in any species. The results presented within this thesis (chapters 2 - 5) address these questions, utilizing the marine fish scup as a model species. Several questions remaining for future research are identified below.

Exposure to TCB elicited effects at many levels in scup. On the most macroscopic level, a high dose of TCB $(5.0 \mathrm{mg} / \mathrm{kg})$ killed many individuals, and the rate of death increased with time following treatment. A small number of individuals apparently escaped the lethal effects of a high TCB dose, and seemed to return toward "normal" histology and biochemistry by day 16 after treatment ( 2 fish). It is unclear, however, whether these two individuals were truly in the process of recovery, or whether they too would have died if given the time.

On a chemical level, the scup were able to metabolize administered TCB, and appeared to depurate the administered dose as both parent compound and as metabolites, largely through a biliary route. However, the circulation distributed TCB throughout the body before such elimination could clear it. The disposition differed at the two doses, with maximal TCB concentrations in liver on day 5 at $0.1 \mathrm{mg} / \mathrm{kg}$ TCB, and on day 12 and 5.0 $\mathrm{mg} / \mathrm{kg}$ TCB. Pharmacokinetic studies have indicated that the time to reach peak concentration is dependent upon the depuration rate constant, and not upon the uptake rate constant. Thus the delayed hepatic peak concentration in fish treated with the high TCB dose suggests slower depuration. Consistent with this suggestion, bile residue data (White et al., in preparation) indicate that fish treated with the low dose had high concentrations of hydroxylated TCB metabolites in bile, whereas fish treated with the high dose had far lower concentrations of TCB metabolites in bile. Impaired metabolism at the high dose was likely related to the reduced hepatic CYP1A1 content in fish treated with this dose, and apparently resulted in TCB accumulation in the livers of fish treated with $5.0 \mathrm{mg} / \mathrm{kg}$ TCB. 
On a tissue level, both TCB doses elicited necrosis of epithelial cells (kidney), altered proliferation of epithelial cells (gill), depletion of lymphoid tissue (kidney), and rearrangement of melanomacrophage cells (kidney, spleen). It is interesting to speculate that some of these tissue alterations may have been mediated by TCB binding to the cytosolic Ah receptor. Other alterations associated with TCB treatment included apparent edema in spleen and kidney, aneurysm of gill tissue, fin erosion, loss of lipid and glycogen stores in liver, and proliferation of endoplasmic reticulum in liver. Individuals treated with the low TCB dose and sampled on day 16 had relatively normal tissue morphology, resembling tissues of control fish. This apparent repair of tissue lesions occurred concomitantly with TCB depuration from tissues. Individuals treated with the high TCB dose exhibited severe tissue pathology until the end of the experiment, displaying no evidence of "recovery", despite the finding that much of the TCB dose had been depurated from these fish by day 16 . At the high dose, it thus appeared that TCB treatment caused mortality before tissue repair and TCB depuration could take place. The proximal cause of death may have been a severe degeneration of kidney and gill tissue. Pathological alterations in liver were similar at the two doses, and included altered hepatocyte composition without necrosis. Thus liver damage cannot explain the apparent suppression of hepatic CYP1A1 protein content in fish treated with the high dose (see below).

We speculate that these TCB-elicited tissue alterations may lead to systemic effects in teleost fish. Prolonged exposure, even to relatively low TCB doses, may compromise excretory and respiratory systems, as was seen in the present study during acute exposure to moderate and high doses. Liver function may also be compromised during long-term exposure, indicated here by an elevation in plasma AST in fish treated with $0.1 \mathrm{mg} / \mathrm{kg}$ TCB and sampled on day 12. Damage to other organs, including gonad, was less severe, although we cannot rule out the possibility of reproductive impairment due to TCB localization in gonad.

On a molecular level, TCB strongly induced CYP1A1 in epithelial and endothelial cells of organs throughout the body. Weak to moderate induction was noted within 24 hours of treatment in every cell type examined, and strong induction was noted at 24 hours in atrial endocardia. CYP1A1 content attenuated by day 16 in many epithelial cell types, but was persistent for several weeks after treatment in the endothelia of every organ examined. CYP1A1 induction in extrahepatic organs may be associated with TCB metabolism in these sites, possibly leading to the in situ production of aqueous-soluble TCB metabolites, reactive TCB metabolites, and reactive oxygen species (see below). As has been suggested in previous work, CYP1A1 induction in extrahepatic endothelia may play a role in binding and sequestration of blood-borne TCB, thus limiting the flow of TCB 
into the organs. This hypothesis was supported by the moderate CYP1A1 induction in capillary endothelia of spleen and bulbus arteriosus, which was seen in fish treated with the high dose but not in fish treated with the low dose. Future studies should define the role of endothelial CYP1A1 in TCB sequestration.

In some epithelial cells CYP1A1 content was greater in fish treated with $0.1 \mathrm{mg} / \mathrm{kg}$ than in fish treated with $5.0 \mathrm{mg} / \mathrm{kg}$, as determined by immunohistochemistry and as verified by immunoblotting: hepatocytes, kidney tubules and collecting ducts, and gill epithelia. In the case of kidney and gill epithelia, reduced CYP1A1 content at the high dose was likely due to general pathology. In the kidneys of fish treated with the high dose, many tubules were characterized by necrotic epithelia, and many others by newly formed, regenerative epithelia. We speculate that the severely necrotic cells and the newly formed cells may have had a lower capacity to express CYP1A1 than did the older, healthier renal tubule cells. Thus the reduced CYP1A1 content at the high dose was related to the pathological alterations in kidney epithelia. In gills of fish treated with the high dose, there was a moderate to diffuse hyperplasia of epithelial cells between the secondary lamellae. The newly formed epithelia did not stain for CYP1A1, and the older epithelia did. Thus the reduced CYP1A1 content in gill epithelia at the high dose was due to the presence of populations of non-staining cells, which existed as a TCB-induced pathology. There was no evidence for marked pathology of liver epithelia at the high dose. We theorize that the reduced CYP1A1 content in livers of fish treated with the high dose was not due to general pathology, but rather resulted from specific TCB/CYP1A1 interactions (see below).

In liver microsomes, CYP1A1 content was three-fold greater in fish treated with the low dose $(0.1 \mathrm{mg} / \mathrm{kg})$ than in fish treated with the high dose $(5.0 \mathrm{mg} / \mathrm{kg})$. Despite the strong difference in content of CYP1A1 protein at the two doses, relative content of CYP1A1 mRNA was the same in livers of fish treated with the two doses. The contents of several other hepatic enzymes were statistically indistinguishable at the two TCB doses: cytochrome b5, NADPH cytochrome c (P450) reductase, UDP-glucuronosyl transferase, cytochrome $\mathrm{P} 450 \mathrm{~B}$, and cytochrome $\mathrm{P} 450 \mathrm{~A}$. Thus the reduced CYP1A1 protein content was found to be post-transcriptionally controlled, and was found to be highly specific for CYP1A1. Both of these findings point toward the hypothesis that "suppressed" CYP1A1 content at the high dose may be associated with specific TCB/CYP1A1 interactions.

On a biochemical level, TCB was found to participate in a complex suite of interactions with CYP1A1. TCB bound to the active site of the enzyme, as demonstrated by binding spectra and by competitive inhibition of EROD activity. In binding, TCB was found to act as an apparent inhibitor of CYP1A1 catalytic activity in liver, kidney, and gill in vivo. TCB was also found to be a substrate of hepatic CYP1A1 in vitro, although it was 
converted to aqueous-soluble metabolites by the enzyme at an exceedingly slow rate. The production of reactive TCB metabolites, those that were capable of binding covalently to microsomal protein, proceeded at an almost negligible rate in vitro. However, we cannot exclude the possibility that reactive metabolite formation contributed to TCB disposition in vivo. It was thus found that TCB bound tightly and specifically to the CYP1A1 active site but was a poor substrate for the enzyme, implying that it may be capable of uncoupling the CYP1A1 catalytic cycle.

The theorized uncoupling was indeed supported by in vitro results. It was found that CYP1A1 was inactivated (a time-dependent loss of catalytic capacity) and denatured (a conversion of $\mathrm{P} 450$ to $\mathrm{P} 420$ ) by TCB, under complete reaction conditions. Inactivation and denaturation were not seen in the absence of TCB nor in the absence of cofactor, implying that substrate binding and electron transport are both required for the inactivation and denaturation. The processes of inactivation and denaturation were enhanced in the presence of sodium azide, suggesting the involvement of a reactive oxygen species such as hydrogen peroxide or hydroxyl radical. Thus TCB, in substrate binding to the CYP1A1 active site, apparently elicited the oxidative inactivation of the enzyme in vitro. We speculate that such oxidative inactivation may contribute to the reduced hepatic CYP1A1 content in fish exposed to a high TCB dose. Future work should further define the mechanisms of the oxidative inactivation, and the potential role of reactive oxygen species in both CYP1A1 degradation and in histopathology in multiple organs. Further work should also focus on drawing a connection between CYP1A1 inactivation by TCB in vitro and the apparent suppression of CYP1A1 protein content by TCB in vivo.

Because TCB exerts profound biochemical changes in teleost fish, interactive effects between TCB and other environmental contaminants are likely. Exposure to low TCB concentrations results in strong CYP1A1 induction, which may lead to increased rates of metabolism of other CYP1A1 substrates in the environment such as PAH and HAH. Increased rates of metabolism may enhance elimination of contaminants from tissues (for example PCBs), but may also result in contaminant activation to carcinogenic forms (for example PAHs such as $\mathrm{BaP}$ ). Exposure to high TCB concentrations results in very weak apparent CYP1A1 induction, manifest as relatively low hepatic CYP1A1 protein content and activity. Such "suppression" of CYP1A1 by TCB may hinder the metabolism, detoxification and elimination of both TCB and other PAH and HAH that are CYP1A1 substrates, and to which the animal is simultaneously exposed. On the other hand, "suppressed" CYP1A1 content would also lower the potential for metabolic activation of other contaminants, such as $\mathrm{BaP}$, to carcinogenic forms. These hypotheses are further complicated by the possibility of competition for binding to the Ah receptor. If an animal is 
exposed to high concentrations of a PAH as well as to TCB, a smaller fraction of the TCB may bind to the Ah receptor than would if the animal were exposed to TCB alone. Thus TCB effects may be minimized in the presence of other environmental Ah receptor agonists.

Synergistic effects may be seen between TCB and environmental contaminants of similar structure. For example, 3,3',4,4',5-HCB may exert a similar CYP1A1 "suppression" at relatively high doses. We thus speculate that CYP1A1 suppression could occur in the presence of low TCB concentrations, in combination with low concentrations of HCB and other similarly acting chemicals. The hepatic TCB concentrations that were associated with CYP1A1 suppression in the present study have been seen in feral fish from contaminated environments, implying that the effects reported here have physiological and environmental relevance. The possibility of synergistic effects with other chemicals increases the likelihood that CYP1A1 suppression could occur in feral fish.

\section{Cetacean studies}

The cytochrome P450 system is known to play a central role in the disposition of toxic organic contaminants in laboratory mammals. However, very little has been determined about cytochrome P450 systems in marine mammals. Marine mammals are heavily exposed to organic contaminants in the environment, thus P450 may play an important role in chemical detoxification and activation in these species. P450 systems have previously been studied in only four cetacean species; the present work includes a catalytic and immunochemical description of P450 systems in two more cetacean species. Furthermore, no direct studies of HAH metabolism have previously been conducted in marine mammals. This thesis provides the first such data, with direct measurements of PCB metabolism in two cetacean species.

Both beluga whale and pilot whale express a hepatic protein with immunochemical similarity to CYP1A1. Correlation analysis indicates that this protein is involved in EROD activity in both species. Content of the CYP1A enzyme is also correlated with PROD and $\mathrm{AHH}$ activities in beluga. In beluga, hepatic content of the CYP1A homologue is positively correlated with body burden of coplanar and mono-ortho substituted PCB, suggesting induction by environmental PCB. Thus the cetacean CYP1A appears to have catalytic and induction properties similar to CYP1A enzymes in laboratory mammals.

Both cetacean species can convert 3,3',4,4'-TCB and 2,2',5,5'-TCB to aqueoussoluble products in vitro, and rates of metabolism vary with sex, species, and congener. Inhibition studies and correlation analysis indicate that CYP1A is involved in the 
metabolism of 3,3'4,4'-TCB in beluga. Both species express hepatic protein(s) with immunochemical similarity to rabbit and dog CYP2B-subfamily enzymes, but without immunochemical similarity to rat CYP2B-subfamily enzymes. The identity and function of these cetacean CYP2B-like proteins is not yet known, and should be the subject of future study.

In summary, this thesis provides several original contributions to the body of work concerning polychlorinated biphenyl toxicity and cytochrome $\mathrm{P} 450$ action in marine fish and marine mammals. The thesis provides a time-course and dose-response study of TCB toxicity in multiple organs of scup, and a time-course and dose-response study of CYP1A1 induction by TCB in multiple organs and cell types of scup. The thesis identifies CYP1A1 as a major catalyst for the metabolism of 3,3',4,4'-TCB in teleost fish, identifies a putative CYP2B form as a major catalyst for the metabolism of 2,2',5,5'-TCB in teleost fish, identifies a CYP1A form as a catalyst involved in the metabolism of 3,3',4,4'-TCB in marine mammals, and identifies the presence of a possible CYP2B homologue in marine mammals. The thesis provides evidence that TCB may act as an uncoupler of the CYP1A1 catalytic cycle in teleost liver in vitro, that such uncoupling is likely associated with CYP1A1 enzyme inactivation and denaturation in vitro, and that such inactivation and denaturation in vitro may be linked to suppressed hepatic CYP1A1 protein content in TCBtreated fish in vivo. Further elucidation of the mechanisms of inactivation and suppression by TCB remain for future work. 


\section{Bibliography}

Addison, R. F., M. C. Sadler and R. A. Lubet (1987). Absence of hepatic microsomal pentyl- or benzyl-resorufin O-dealkylase induction in rainbow trout (Salmo gairdneri) treated with phenobarbitone. Biochem. Pharmacol. 36: 1183-1184.

Addison, R. F., M. E. Zinc and D. E. Willis (1977). Mixed-function oxidase in trout liver: absence of induction following feeding of p,p'-DDT or p,p'-DDE. Comp. Biochem. Physiol. 57C: 39-43.

Alvares, A. P., D. R. Bickers and A. Kappas (1973). Polychlorinated biphenyls: a new type of inducer of cytochrome P-448 in the liver. Proc. Nat. Acad. Sci. USA 70: 13211325.

Andersson, T., M. Pesonen and C. Johansson (1985). Differential induction of cytochrome P-450-dependent monooxygenase, epoxide hydrolase, glutathione transferase, and UDP glucuronyltransferase activities in the liver of the rainbow trout by Bnaphthoflavone or Clophen A50. Biochem. Pharmacol. 34: 3309-3314.

Ankley, G. T., R. E. Reinert, R. T. Meyer, M. D. Burke and M. Agosin (1987). Metabolism of alkoxyphenoxazones by channel catfish liver microsomes: Effects of phenobarbital, Aroclor 1254, and 3-methylcholanthrene. Biochem. Pharmacol. 36: 13791381.

Arias, I. M., D. Doyle and R. T. Schimke (1969). Studies on the synthesis and degradation of protein of the endoplasmic reticulum of rat liver. J. Biol. Chem. 244: 3303-3315.

Bach, J. and J. Snegaroff (1989). Effects of the fungicide prochloraz on xenobiotic metabolism in rainbow trout: in vivo induction. Xenobiotica 19: 1-9.

Bend, J. R., R. J. Pohl, N. P. Davidson and J. R. Fouts (1974). Response of hepatic and renal microsomal mixed-function oxidases in the little skate, Raja erinacea, to pretreatment with 3-methylcholanthrene or TCDD (2,3,7,8-tetrachlorodibenzo-p-dioxin). Bull. Mt. Des. Isl. Biol. Lab. 14: 7-12.

Binder, R. L. (1982). Xenobiotic monooxygenase activity and the response to inducers of cytochrome P-450 during embryonic and larval development in fish. MIT/WHOI, Ph.D. Thesis.

Birnbaum, L. (1985). The role of structure in the disposition of halogenated aromatic xenobiotics. Environ. Health Perspect. 61: 11-20.

Black, M. C. and J. F. McCarthy (1990). Effects of sublethal exposure to chlorine on the uptake of polychlorinated biphenyl congeners by rainbow trout, Salmo gairdneri (Richardson). Aquat. Toxicol. 17: 275-290.

Black, S. D. and M. J. Coon (1986). Comparative structures of P-450 cytochromes. Cytochrome P-450 Structure, Mechanism, and Biochemistry. Ortiz de Montellano, P. R. (ed.). New York, Plenum Press. 161-216. 
Boon, J. P., F. Eijgenraam, J. M. Everaarts and J. C. Duinker (1989). A structure-activity relationship (SAR) approach towards metabolism of PCBs in marine animals from different trophic levels. Marine Environ. Res. 27: 159-176.

Borlakoglu, J. T. and J. P. G. Wilkins (1993). Metabolism of di-, tri-, tetra-, penta- and hexachlorobiphenyls by hepatic microsomes isolated from control animals and animals treated with Aroclor 1254, a commercial mixture of polychlorinated biphenyls (PCBs). Comp. Biochem. Physiol. 105C: 95-106.

Brouwer, A., P. J. H. Reijnders and J. H. Koeman (1989). Polychlorinated biphenyl (PCB)-contaminated fish induces vitamin A and thyroid hormone deficiency in the common seal (Phoca vitulina). Aquat. Toxicol. 15: 99-106.

Brouwer, A. and K. J. van den Berg (1986). Binding of a metabolite of 3,4,3',4'tetrachlorobiphenyl to transthyretin reduces serum vitamin A transport by inhibiting the formation of the protein complex carrying both retinol and thyroxin. Toxicol. Appl. Pharmacol. 85: 301-312.

Brown, J. F. (1992). Metabolic alterations of PCB residues in aquatic fauna: distributions of cytochrome P4501A- and P4502B- like activities. Marine Environ. Res. 34: 261-266.

Buchmann, A., R. Wannemacher, E. Kulzer, D. R. Buhler and K. W. Bock (1993). Immunohistochemical Localization of the Cytochrome P450 Isozymes LMC2 and LM4B (P4501A1) in 2,3,7,8-tetrachlorodibenzo-p-dioxin- treated zebrafish (Brachydanio rerio). Toxicol. Appl. Pharmacol. 123: 160-169.

Burke, M. D., S. Thompson, C. R. Elcombe, J. Halpert, T. Haaparanta and R. T. Mayer (1985). Ethoxy-, pentoxy- and benzyloxyphenoxazones and homologues: a series of substrates to distinguish between different induced cytochromes P-450. Biochem. Pharmacol. 34: 3337-3345.

Burns, K. A. (1976). Microsomal mixed function oxidases in an estuarine fish, Fundulus heteroclitus, and their induction as a result of environmental contamination. Comp. Biochem. Physiol. 53B: 443-446.

Casillas, E., J. Sundqvist and W. E. Ames (1982). Optimization of assay conditions for, and selected tissue distribution of alanine aminotransferase and aspartate aminotransferase of, English sole (Parophrys vetulus girard). J. Fish Biol. 21: 197-204.

Celander, M., D. R. Buhler, L. Forlin, A. Goksoyr, C. L. Miranda, B. R. Woodin and J. J. Stegeman (1994). Immunochemical comparison of constitutively expressed cytochrome P450 isoforms from different teleost fish species and their relatedness to human CYP3A4 (abstract). Tenth International Symposium on Microsomes and Drug Oxidations, Toronto. 292.

Celander, M. and L. Forlin (submitted). Decreased responsiveness of the hepatic cytochrome P4501A1 system in rainbow trout (Oncorhynchuss mykiss) after prolonged exposure to PCB. Aquat. Toxicol.

Chang, T. K. H., F. J. Gonzalez and D. J. Waxman (1994). Evaluation of triacetyloleandomycin, alpha-naphthoflavone and diethyldithiocarbamate as selective chemical probes for inhibition of human cytochromes P450. Arch. Biochem. Biophys. 311: $437-442$. 
Chiou, C. T. (1985). Partition coefficients of organic compounds in lipid-water systems and correlations with fish bioconcentration factors. Environ. Sci. Technol. 19: 57-62.

Chirgwin, J. M., A. E. Przybyla, R. J. MacDonald and W. J. Rutter (1979). Isolation of biologically active ribonucleic acid from sources enriched in ribonuclease. Biochemistry 18: 5294-5299.

Clemens, M. J. (1986). Extraction of RNA by the guanidinium thiocyanate procedure. Transcription and Translation: a Practical Approach. Hames, B. D. and S. J. Higgins (ed.). Oxford, IRL Press. 217.

Collier, E. (1943). Poisoning by chlorinated naphthalene. Lancet Jan. 16: 72-74.

de Boer, J., C. J. N. Stronck, W. A. Traag and J. van der Meer (1993). Non-ortho and mono-ortho substituted chlorobiphenyls and chlorinated dibenzo-p-dioxins and dibenzofurans in marine and freshwater fish and shellfish from the Netherlands. Chemosphere 26: 1823-1842.

De Matteis, F., S. J. Dawson, A. R. Boobis and A. Comoglio (1991). Inducible bilirubindegrading system of rat liver microsomes: role of cytochrome P4501A1. Mol. Pharmacol. 40: 686-691.

De Matteis, F., S. J. Dawson and A. H. Gibbs (1993). Two pathways of iron-catalyzed oxidation of bilirubin: effect of desferrioxamine and trolox, and comparison with microsomal oxidation. Free Radic. Biol. Med. 15: 301-309.

De Matteis, F., T. Trenti, A. H. Gibbs and J. B. Greig (1989). Inducible bilirubindegrading system in the microsomal fraction of rat liver. Mol. Pharmacol. 35: 831-838.

Dey, W. P., T. H. Peck, C. E. Smith and G. L. Kreamer (1993). Epizoology of hepatic neoplasia in Atlantic tomcod (Microgadus tomcod) from the Hudson River estuary. Can. J. Fish. Aquat. Sci. 50: 1897-1907.

Duignan, D. B., I. G. Sipes, T. B. Leonard and J. R. Halpert (1987). Purification and characterization of the dog hepatic cytochrome $P-450$ isozyme responsible for the metabolism of 2,2',4,4',5,5'-hexachlorobiphenyl. Arch. Biochem. Biophys. 255: 290303.

Duinker, J. C., M. T. J. Hillebrand, T. Zeinstra and J. P. Boon (1989). Individual chlorinated biphenyls and pesticides in tissues of some cetacean species from the North Sea and the Atlantic Ocean; tissue distribution and biotransformation. Aquat. Mamm. 15: 95124.

Durham, S. K. and A. Brouwer (1989). 3,4,3',4,'-Tetrachlorobiphenyl-induced effects in the rat liver. II. Electron microscopic autoradiographic localization of ${ }^{3} \mathrm{H}-\mathrm{TCB}$. Toxicol. Pathol. 17: 782-788.

Eisele, T. A., R. A. Coulombe, N. E. Pawlowski and J. E. Nixon (1984). The effects of route of exposure and combined exposure of mixed function oxidase inducers and suppressors on hepatic parameters in rainbow trout (Salmo gairdneri). Aquat. Toxicol. 5: 211-226. 
Elskus, A. A. and J. J. Stegeman (1989). Further consideration of phenobarbital effects on cytochrome P-450 activity in the killifish, Fundulus heteroclitus. Comp. Biochem.

Physiol. C 92C: 223-230.

Elskus, A. A., J. J. Stegeman, L. C. Susani, D. Black, R. J. Pruell and S. J. Fluck (1989). Polychlorinated biphenyls concentration and cytochrome P-450E expression in winter flounder from contaminated environments. Marine Environ. Res. 28: 25-30.

Ernster, L. and S. Orrenius (1965). Substrate-induced synthesis of the hydroxylating enzyme system of liver microsomes. Fed. Proc. 24: 1190-1199.

Evans, D. H. (1987). The fish gill: site of action and model for toxic effects of environmental pollutants. Environ. Health Perspect. 71: 47-58.

Ferguson, H. W. (1989). Systemic Pathology of Fish: A Text and Atlas of Comparative Tissue Responses in Diseases of Teleosts. Ames, Iowa State University Press. 263 pages.

Forlin, L. and U. Lidman (1978). Effects of Clophen A50, 4-, 2,5,2',5'-tetra and $2,4,5,2^{\prime}, 4^{\prime}, 5^{\prime}$-hexachlorobiphenyl on the mixed-function oxidase system of rainbow trout (Salmo gairdnerii Rich.) liver. Comp. Biochem. Physiol. 60C: 193-197.

Fowler, S. W. (1990). Critical review of selected heavy metal and chlorinated hydrocarbon concentrations in the marine environment. Marine Environ. Res. 29: 1-64.

Frey, A. B., D. J. Waxman and F. Kreibich (1985). The structure of phenobarbitalinducible rat liver cytochrome P-450 isoenzyme PB-4. Production and characterization of site-specific antibodies. J. Biol. Chem. 260: 15253-15265.

Ghiasuddin, S. M., R. E. Menzer and J. O. Nelson (1976). Metabolism of 2,5,2'trichloro-, 2,5,2',5',-tetrachloro-, and 2,4,2',5'-pentachlorobiphenyl in rat hepatic microsomal systems. Toxicol. Appl. Pharmacol. 36: 187-194.

Goksøyr, A., T. Andersson, L. Forlin, E. A. Snowberger, B. R. Woodin and J. J. Stegeman (1989). Cytochrome P-450 monooxygenase activity and immunochemical properties of adult and foetal piked whales, Balaenoptera acutorostrata. Cytochrome P-450. Biochemistry and Biophysics. Schuster, I. (ed.). Taylor \& Francis. 698-701.

Goksøyr, A., T. Andersson, L. Forlin, J. Stenersen, E. A. Snowberger, B. R. Woodin and J. J. Stegeman (1988). Xenobiotic and steroid metabolism in adult and foetal piked (minke) whales, Balaenoptera acutorostrata. Marine Environ. Res. 24: 9-13.

Goksøyr, A., T. Andersson, T. Hansson, J. Klungsoyr, Y. Zhang and L. Forlin (1987). Species characteristics of the hepatic xenobiotic and steroid biotransformation systems of two teleost fish, Atlantic cod (Gadus morhua) and rainbow trout (Salmo gairdneri).

Toxicol. Appl. Pharmacol. 89: 347-360.

Goksøyr, A., J. E. Solbakken, J. Tarleb $\varnothing$ and J. Klungsøyr (1986). Initial characterization of the hepatic microsomal cytochrome P-450-system of the piked whale (minke)

Balaenoptera acutorostrata. Marine Environ. Res 19: 185-203.

Goldstein, J. A. and S. Safe (1989). Mechanism of action and structure-activity relationships for the chlorinated dibenzo-p-dioxins and related compounds. Halogenated biphenyls, terphenyls, naphthalenes, dibenzodioxins and related products. Kimbrough, R. 
D. and A. A. Jensen (ed.). Amsterdam, Elsevier Science Publishers, Biomedical Division. 239-293.

Gooch, J. W., A. A. Elskus, P. J. Kloepper-Sams, M. E. Hahn and J. J. Stegeman (1989). Effects of ortho and non-ortho substituted polychlorinated biphenyl congeners on the hepatic monooxygenase system in scup (Stenotomus chrysops). Toxicol. Appl. Pharmacol. 98: 422-433.

Gray, E. S. (1988). Sexual patterns of monooxygenase function in the liver of marine teleosts and the regulation of activity by estradiol. MIT/WHOI, Ph.D. Thesis.

Guengerich, F. P. (1978). Destruction of heme and hemoproteins mediated by liver microsomal reduced nicotinamide adenine dinucleotide phosphate-cytochrome P-450 reductase. Biochemistry 17: 3633-3639.

Guiney, P. D., R. E. Peterson, M. J. Melancon and J. J. Lech (1977). The distribution and elimination of 2,5,2',5'-[14C]tetrachlorobiphenyl in rainbow trout (Salmo gairdneri). Toxicol. Appl. Pharmacol. 39: 329-338.

Gutman, Y. and M. Kidron (1971). Liver $N$-demethylating activity - temperature effect and phenobarbital induction in different species. Biochem. Pharmacol. 20: 3547-3550.

Haasch, M. L., W. K. Graf, E. M. Quardokus, R. T. Mayer and J. J. Lech (1994). Use of 7-alkoxyphenoxazones, 7-alkoxycoumarins and 7-alkoxyquinolines as fluorescent substrates for rainbow trout hepatic microsomes after treatment with various inducers. Biochem. Pharmacol. 47: 893-903.

Hacking, M. A., J. Budd and K. Hodson (1978). The ultrastructure of the liver of the rainbow trout: normal structure and modifications after chronic administration of a polychlorinated biphenyl Aroclor 1254. Can. J. Zool. 56: 477-491.

Hahn, M. E., T. M. Lamb, M. E. Schultz, R. M. Smolowitz and J. J. Stegeman (1993). Cytochrome P4501A induction and inhibition by 3,3',4,4'-tetrachlorobiphenyl in an Ah receptor-containing fish hepatoma cell line (PLHC-1). Aquat. Toxicol. 26: 185-208.

Hahn, M. E., A. Poland, E. Glover and J. J. Stegeman (1994). Photoaffinity labeling of the Ah receptor: Phylogenetic survey of diverse vertebrate and invertebrate species. Arch. Biochem. Biophys. 310: 218-228.

Hahn, M. E. and J. J. Stegeman (1994). Regulation of cytochrome P4501A1 in teleosts: sustained induction of CYP1A1 mRNA, protein, and catalytic activity by 2,3,7,8tetrachlorodibenzofuran in the marine fish Stenotomus chrysops. Toxicol. Appl. Pharmacol. 127: 187-198.

Halliwell, B. and J. M. C. Gutteridge (1986). Oxygen free radicals and iron in relation to biology and medicine: some problems and concepts. Arch. Biochem. Biophys. 246: 501514.

Hampton, J. A., R. C. Lantz and D. E. Hinton (1989). Functional units in rainbow trout (Salmo gairdneri, Richardson) Liver: III. Morphometric analysis of parenchyma, stroma, and component cell types. Amer. J. Anat. 185: 58-73. 
Hargraves, W. A. and J. R. Allen (1979). The in vitro binding of 2,2',5,5'tetrachlorobiphenyl metabolites to rat liver microsomal proteins. Res. Commun. Chem. Pathol. Pharmacol. 25: 33-52.

Hawkes, C. L. and L. A. Norris (1977). Chronic oral toxicity of 2,3,7,8tetrachlorodibenzo-p-dioxin. Trans. Am. Fish. Soc. 106: 641-645.

Helrich, K. (1990). Official Methods of Analysis of the Association of Official Analytical Chemists, 15th edition. Helrich, K. (ed.). Arlington, VA, Association of Official Analytical Chemists. 283-284.

Herbst, E., I. Scheunert, W. Klein and F. Korte (1978). Uptake and conversion of 2,5,4'trichlorobiphenyl- ${ }^{14} \mathrm{C}, 2,4,6,2$ ', 4 '-pentachlorobiphenyl- ${ }^{14} \mathrm{C}$ and chloroalkylene-9- ${ }^{14} \mathrm{C}$ by goldfish after a single water treatment. Chemosphere 7: 221-230.

Hesse, S., M. Mezger and T. Wolff (1978). Activation of [14C] chlorobiphenyls to protein-binding metabolites by rat liver microsomes. Chem.-Biol. Interact. 20: 355-365.

Hesse, S. and T. Wolff (1977). In vitro interactions of di-, tetra- and hexa-chlorobiphenyl with rabbit liver monooxygenase. Biochem. Pharmacol. 26: 2043-2047.

Hinz, R. and F. Matsumura (1977). Comparative metabolism of PCB isomers by three species of fish and the rat. Bull. Environ. Contam. Toxicol. 18: 631-639.

Hollander, M. C. and A. J. Fornace (1990). Estimation of relative mRNA content by filter hybridization to a polythymidylate probe. BioTechniques 9: 174-179.

Hutzinger, O., D. M. Nash, S. Safe, A. S. W. DeFreitas, R. J. Norstrom, D. J. Wildish and V. Zitko (1972). Polychlorinated biphenyls: metabolic behavior of pure isomers in pigeons, rats, and brook trout. Science 178: 312-313.

Ingelman-Sundberg, M. (1986). Cytochrome P-450 organization and membrane interactions. Cytochrome P-450 Structure, Mechanism, and Biochemistry. Ortiz de Montellano, P. R. (ed.). New York, Plenum Press. 119-160.

Ishida, C., N. Koga, N. Hanioka, H. K. Saeki and H. Yoshimura (1991). Metabolism in vitro of 3,4,3',4'- and 2,5,2',5'-tetrachlorobiphenyl by rat liver microsomes and highly purified cytochrome P450. J. Pharmacobio.-Dyn. 14: 276-284.

Jensen, E. G., J. U. Skaare, E. Egaas and A. Goksoyr (1991). Response of xenobiotic metabolizing enzymes in rainbow trout (Oncorhynchus mykiss) to endosulfan, detected by enzyme activities and immunochemical methods. Aquat. Toxicol. 21: 81-92.

Jensen, S. (1966). Report of a new chemical hazard. New Scientist 32: 612.

Jones, P. A., R. J. Sloan and M. P. Brown (1989). PCB congeners to monitor with caged juvenile fish in the upper Hudson River. Environ. Toxicol. Chem. 8: 793-803.

Kaminsky, L. S., M. W. Kennedy, S. M. Adams and F. P. Guengerich (1981). Metabolism of dichlorobiphenyls by highly purified isozymes of rat liver cytochrome P450. Biochemistry 20: 7379-7384. 
Karuzina, I. I. and A. I. Archakov (1994). The oxidative inactivation of cytochrome P450 in monooxygenase reactions. Free Radic. Biol. Med. 16: 73-97.

Kennedy, M. W., N. K. Carpentier, P. O. Dymerski and L. S. Kaminsky (1981). Metabolism of dichlorobiphenyls by hepatic microsomal cytochrome P-450. Biochem. Pharmacol. 30: 577-588.

Kennedy, S. W., A. Lorenzen, C. A. James and B. T. Collins (1993). Ethoxyresorufin-Odeethylase and porphyrin analysis in chicken embryo hepatocyte cultures with a fluorescence multi-well plate reader. Anal. Biochem. 211: 102-112.

Klasson Wehler, E., A. Bergman, I. Brandt, P. O. Darnerud and C. A. Wachtmeister (1989). 3,3',4,4'-Tetrachlorobiphenyl excretion and tissue retention of hydroxylated metabolites in the mouse. Drug Metab. Dispos. 17: 441-448.

Klasson Wehler, E., B. Brunstrom, U. Rannug and A. Bergman (1990). 3,3',4,4'Tetrachlorobiphenyl: metabolism by the chick embryo in ovo and toxicity of hydroxylated metabolites. Chem.-Biol. Interact. 73: 121-132.

Klaunig, J. E., M. M. Lipsky, B. F. Trump and D. E. Hinton (1979). Biochemical and ultrastructural changes in teleost liver following subacute exposure to PCB. J. Environ. Pathol. Toxicol. 2: 953-963.

Kloepper-Sams, P. J., S. S. Park, H. V. Gelboin and J. J. Stegeman (1987). Specificity and cross-reactivity of monoclonal and polyclonal antibodies against cytochrome P450E of the marine fish scup. Arch. Biochem. Biophys. 253: 268-278.

Kloepper-Sams, P. J. and J. J. Stegeman (1989). The temporal relationships between cytochrome $\mathrm{P}-450 \mathrm{E}$ protein content, catalytic activity and mRNA levels in the teleost Fundulus heteroclitus following treatment with ß-naphthoflavone. Arch. Biochem. Biophys. 268: 525-535.

Kloepper-Sams, P. J. and J. J. Stegeman (1994). Turnover of hepatic microsomal cytochrome P4501A protein and heme in B-naphthoflavone-induced Fundulus heteroclitus. Molec. Mar. Biol. Biotech. 3: 171-183.

Klotz, A. V., J. J. Stegeman and C. Walsh (1983). An aryl hydrocarbon hydroxylating hepatic cytochrome P-450 from the marine fish Stenotomus chrysops. Arch. Biochem. Biophys. 226: 578-592.

Klotz, A. V., J. J. Stegeman and C. Walsh (1984). An alternative 7-ethoxyresorufin Odeethylase activity assay: a continuous visible spectrophotometric method for measurement of cytochrome P-450 monoxygenase activity. Anal. Biochem. 140: 138-145.

Kodavanti, P. R. S., D.-S. Shin, H. A. Tilson and G. J. Harry (1993). Comparative effects of two polychlorinated biphenyl congeners on calcium homeostasis in rat cerebellar granule cells. Toxicol. Appl. Pharmacol. 123: 97-106.

Korach, K. S., P. Sarver, K. Chae, J. A. McLachlan and J. D. McKinney (1988).

Estrogen receptor-binding activity of polychlorinated hydroxybiphenyls: Conformationally restricted structural probes. Mol. Pharmacol. 33: 120-126. 
Kuriyama, Y., T. Omura, P. Siekevitz and G. E. Palade (1969). Effects of phenobarbital on the synthesis and degradation of the protein components of rat liver microsomal membranes. J. Biol. Chem. 244: 2017-2026.

Kuthan, H. and V. Ullrich (1982). Oxidase and oxygenase function of the microsomal cytochrome P-450 monooxygenase system. Eur. J. Biochem. 126: 583-588.

Lambrecht, R. W., P. R. Sinclair, W. J. Bement and J. F. Sinclair (1988). Uroporphyrin accumulation in cultured chick embryo hepatocytes: comparison of 2,3,7,8-

tetrachlorodibenzo-p-dioxin and 3,4,3',4'-tetrachlorobiphenyl. Toxicol. Appl. Pharmacol. 96: $507-516$.

Lindstrom-Seppa, P., P. J. Korytko, M. E. Hahn and J. J. Stegeman (1994). Uptake of waterborne 3,3',4,4'-tetrachlorobiphenyl and organ and cell-specific induction of cytochrome P4501A in adult and larval fathead minnow Pimephales promelas. Aquat. Toxicol. 28: 147-167.

Loosemore, M., D. R. Light and C. Walsh (1980). Studies on the autoinactivation behavior of pure, reconstituted phenobarbital-induced cytochrome P-450 isozyme from rat liver. J. Biol. Chem. 255: 9017-9020.

Lorenzana, R. M., O. R. Hedstrom and D. R. Buhler (1988). Localization of cytochrome $\mathrm{P}-450$ in the head and trunk kidney of rainbow trout (Salmo gairdneri). Toxicol. Appl. Pharmacol. 96: 159-167.

Lorenzen, A. and S. W. Kennedy (in preparation). Effects of halogenated aromatic hydrocarbons on cytochrome P-4501A activity and induction in chicken embryo hepatocytes.

Luna, L. G. (1968). Manual of histologic staining methods of the Armed Forces Institute of Pathology. NY, McGraw-Hill. 258 pages.

Matthews, H. B. and D. B. Tuey (1980). The effect of chlorine position on the distribution and excretion of four hexachlorobiphenyl isomers. Toxicol. Appl. Pharmacol. 53: 377388.

McDermott-Ehrlich, D. J., M. J. Sherwood, T. C. Heesen, D. R. Young and A. J. Mearns (1977). Chlorinated hydrocarbons in Dover sole, Microstomus pacificus: local migrations and fin erosion. Fish. Bull. 75: 513-517.

McFarland, V. A. and J. U. Clark (1989). Environmental occurrence, abundance, and potential toxicity of polychlorinated biphenyl congeners: considerations for a congenerspecific analysis. Environ. Health Perspect. 81: 225-239.

McMurry, T. J. and J. T. Groves (1986). Metalloporphyrin models for cytochrome P-450. Cytochrome P-450 Structure, Mechanism, and Biochemistry. Ortiz de Montellano, P. R. (ed.). New York, Plenum Press. 1-28.

Melancon, M. J. and J. J. Lech (1976). Isolation and identification of a polar metabolite of tetrachlorobiphenyl from bile of rainbow trout exposed to ${ }^{14} \mathrm{C}$-tetrachlorobiphenyl. Bull. Environ. Contam. Toxicol. 15: 181-188. 
Melancon, M. J. and J. J. Lech (1983). Dose-effect relationship for induction of hepatic monooxygenase activity in rainbow trout and carp by Aroclor 1254. Aquat. Toxicol. 4: $51-61$.

Melancon, M. J., B. A. Rattner and L. J. LeCaptain (1990). Comparative induction of cytochromes $\mathrm{P} 450$ by 3-methylcholanthrene (MC) and phenobarbital (PB) in mallard duck (MD) and in Fisher-344 rat (FR) (abstract). Annual Meeting of the Society of Environmental Toxicology and Chemistry, Arlington, VA. P160.

Metcalf, R. L., J. R. Sanborn, P.-Y. Lu and D. Nye (1975). Laboratory model ecosystem studies of the degradation and fate of radiolabeled tri-, tetra-, and pentachlorobiphenyl compared with DDE. Arch. Environ. Contam. Toxicol. 3: 151-165.

Miller, M. R., D. E. Hinton, J. J. Blair and J. J. Stegeman (1988). Immunohistochemical localization of cytochrome P450E in liver, gill and heart of scup (Stenotomus chrysops) and rainbow trout (Salmo gairdneri). Marine Environ. Res. 24: 37-39.

Miller, M. R., D. E. Hinton and J. J. Stegeman (1989). Cytochrome P-450E induction and localization in gill pillar (endothelial) cells of scup and rainbow trout. Aquat. Toxicol. 14: 307-322.

Miller, R. A., L. A. Norris and C. L. Hawkes (1973). Toxicity of 2,3,7,8tetrachlorodibenzo-p-dioxin (TCDD) in aquatic organisms. Environ. Health Perspect. 5: 177-186.

Mills, R. A., C. D. Millis, G. A. Dannan, F. P. Guengerich and S. D. Aust (1985). Studies on the structure-activity relationships for the metabolism of polybrominated biphenyls by rat liver microsomes. Toxicol. Appl. Pharmacol. 78: 96-104.

Miranda, C. L., J.-L. Wang, H.-S. Chang and D. R. Buhler (1990). Multiple effects of $3,4,5,3^{\prime}, 4^{\prime}, 5^{\prime}$-hexachlorobiphenyl administration on hepatic cytochrome P450 isozymes and associated mixed-function oxidase activities in rainbow trout. Biochem. Pharmacol. 40: $387-390$.

Monosson, E. and J. J. Stegeman (1991). Cytochrome P450E (P450IA) induction and inhibition in winter flounder by 3,3',4,4'-tetrachlorobiphenyl: comparison of response in fish from Georges Bank and Narragansett Bay. Environ. Toxicol. Chem. 10: 765-774.

Morales, N. M. and H. B. Matthews (1979). In vivo binding of 2,3,6,2',3',6'hexachlorobiphenyl and 2,4,5,2',4',5'-hexachlorobiphenyl to mouse liver macromolecules. Chem.-Biol. Interact. 27: 99-110.

Morrison, H. G., M. F. Oleksiak, N. W. Cornell, M. L. Sogin and J. J. Stegeman (submitted). Identification of cytochrome P4501A genes from two teleost fish, toadfish (Opsanus tau) and scup (Stenotomus chrysops), and phylogenetic analysis of CYP1A genes. Biochem. J.

Muir, D. C., C. A. Ford, R. E. A. Stewart, T. G. Smith, R. F. Addison, M. E. Zinck and P. Beland (1990). Organochlorine contaminants in belugas, Delphinapterus leucas, from Canadian waters. Can. Bull. Fish. Aquat. Sci. 224: 165-190.

Muir, D. C. G., R. Wagemann, N. P. Grift, R. J. Norstrom, M. Simon and J. Lien (1988). Organochlorine chemical and heavy metal contaminants in white-beaked dolphins 
(Lagenorhynchus albirostris) and pilot whales (Globicephala melaena) from the coast of Newfoundland, Canada. Arch. Environ. Contam. Toxicol. 17: 613-629.

Murchelano, R. A. (1975). The histopathology of fin rot disease in winter flounder from the New York Bight. J. Wildlife Dis. 11: 263-267.

Myers, M. S., O. P. Olson, L. L. Johnson, C. S. Stehr, T. Hom and U. Varanasi (1992). Hepatic lesions other than neoplasms in subadult flatfish from Puget Sound, Washington: relationships with indices of contaminant exposure. Marine Environ. Res. 34: 45-51.

Narbonne, J. F. (1979). Polychlorinated biphenyl accumulation in grey mullets (Chelon labrosus): effect of age. Bull. Environ. Contam. Toxicol. 22: 65-68.

Nebert, D. W. and F. J. Gonzalez (1987). P450 genes: structure, evolution, and regulation. Ann. Rev. Biochem. 56: 945-993.

Nelson, D. R., T. Kamataki, D. J. Waxman, F. P. Guengerich, R. W. Estabrook, R. Feyereisen, F. J. Gonzalez, M. J. Coon, I. C. Gunsalus, O. Gotoh, K. Okuda and D. W. Nebert (1993). The P450 superfamily - update on new sequences, gene mapping, accession numbers, early trivial names of enzymes, and nomenclature. DNA Cell Biol. 12: $1-51$.

Niimi, A. J. and B. G. Oliver (1983). Biological half-lives of polychlorinated biphenyl (PCB) congeners in whole fish and muscle of rainbow trout (Salmo gairdneri). Can. J. Fish. Aquat. Sci. 40: 1388-1394.

Nordblom, G. D. and M. J. Coon (1977). Hydrogen peroxide formation and stoichiometry of hydroxylation reactions catalyzed by highly purified liver microsomal cytochrome P450. Arch. Biochem. Biophys. 180: 343-347.

Norstrom, R. J., D. C. G. Muir, C. A. Ford, M. Simon, C. R. Macdonald and P. Beland (1992). Indications of P450 monooxygenase activities in beluga (Delphinapterus leucas) and narwhal (Monodon monoceros) from patterns of PCB, PCDD and PCDF accumulation. Marine Environ. Res. 34: 267-272.

Norstrom, R. J., M. Simon and D. C. G. Muir (1990). Polychlorinated dibenzo-p-dioxins and dibenzofurans in marine mammals in the Canadian north. Environ. Poll. 66: 1-19.

O'Connor, J. M. and J. C. Pizza (1987). Dynamics of polychlorinated biphenyls in striped bass from the Hudson River. III. Tissue Disposition and routes for elimination. Estuaries 10: $68-77$.

Okada, Y. and T. Omura (1978). Synthesis and turnover of microsomal and mitochondrial NADH-cytochrome b5 reductases in rat liver. J. Biochem. 83: 1039-1048.

Omura, T. and R. Sato (1964). The carbon monoxide-binding pigment of liver microsomes. J. Biol. Chem. 239: 2370-2378.

Omura, T., P. Siekevitz and G. E. Palade (1967). Turnover of constituents of the endoplasmic reticulum membranes of rat hepatocytes. J. Biol. Chem. 242: 2389-2396.

Ortiz de Montellano, P. R. (1986). Oxygen activation and transfer. Cytochrome P-450 Structure. Mechanism, and Biochemistry. Ortiz de Montellano, P. R. (ed.). New York, Plenum Press. 217-271. 
Ortiz de Montellano, P. R. and N. O. Reich (1986). Inhibition of cytochrome P-450 enzymes. Cytochrome P-450 Structure, Mechanism, and Biochemistry. Ortiz de Montellano, P. R. (ed.). New York, Plenum Press. 273-314.

Park, S. S., H. Miller, A. V. Klotz, P. J. Kloepper-Sams, J. J. Stegeman and H. V. Gelboin (1986). Monoclonal antibodies to liver microsomal cytochrome P-450E of the marine fish Stenotomus chrysops (scup): cross-reactivity with 3-methylcholanthrene induced rat cytochrome P-450. Arch. Biochem. Biophys. 249: 339-350.

Parkinson, A., L. Robertson, L. Safe and S. Safe (1980). Polychlorinated biphenyls as


30: $271-285$.

Parkinson, A., S. H. Safe, L. W. Robertson, P. E. Thomas, D. E. Ryan, L. M. Reik and W. Levin (1983). Immunochemical quantitation of cytochrome P-450 isozymes and epoxide hydrolase in liver microsomes from polychlorinated or polybrominated biphenyltreated rats. J. Biol. Chem. 258: 5967-5976.

Peterson, J. A. and R. A. Prough (1986). Cytochrome P-450 reductase and cytochrome b5 in cytochrome P-450 catalysis. Cytochrome P-450 Structure, Mechanism, and Biochemistry. Ortiz de Montellano, P. R. (ed.). New York, Plenum Press. 89-118.

Pierce, K. V., B. B. McCain and S. R. Wellings (1978). Pathology of hepatomas and other liver abnormalities in English sole (Parophrys vetulus) from the Duwamish River estuary, Seattle, Washington. J. Nat. Cancer Inst. 60: 1445-1453.

Poland, A. and E. Glover (1974). Comparison of 2,3,7,8-tetrachlorodibenzo-p-dioxin, a potent inducer of aryl hydrocarbon hydroxylase, with 3-methyl-cholanthrene. $\underline{\mathrm{Mol}}$. Pharmacol. 10: 349-359.

Poland, A., P. Teitelbaum and E. Glover (1989). [ $\left.{ }^{125} \mathrm{I}\right] 2$-Iodo-3,7,8-trichlorodibenzo-pdioxin-binding species in mouse liver induced by agonists for the Ah receptor:

characterization and identification. Mol. Pharmacol. 36: 113-120.

Rao, C. V. and S. A. Banerji (1990). Effect of polychlorinated biphenyl (Aroclor 1260) on histology of kidney and thyroid of rats. Indian J. Exp. Biol. 28: 152-154.

Reijnders, P. J. H. (1986). Reproductive failure in common seals feeding on fish from polluted coastal waters. Nature 324: 456-457.

Reinke, L. A., S. M. Bailey, J. M. Rau and P. B. McCay (1994). Oxygen radical formation in well-washed rat liver microsomes: spin trapping studies. Free Radic. Res. 20: $51-60$.

Risebrough, R. W., P. Rieche, D. B. Peakall, S. G. Herman and M. N. Kirven (1968). Polychlorinated biphenyls in the global ecosystem. Nature 220: 1098-1102.

Rodman, L. E., S. I. Shedlofsky, A. T. Swim and L. W. Robertson (1989). Effects of polychlorinated biphenyls on cytochrome P450 induction in the chick embryo hepatocyte culture. Arch. Biochem. Biophys. 275: 252-262. 
Ryan, J. J., D. Levesque, L. G. Panopio, W. F. Sun, Y. Masuda and H. Kuroki (1993). Elimination of polychlorinated dibenzofurans (PCDFs) and polychlorinated biphenyls (PCBs) from human blood in the Yusho and Yu-Cheng rice oil poisonings. Arch. Environ. Contam. Toxciol. 24: 504-512.

Safe, S. (1984). Polychlorinated biphenyls (PCBs) and polybrominated biphenyls (PBBs): biochemistry, toxicology, and mechanism of action. CRC Crit. Rev. Toxicol. 13: 319395.

Safe, S. (1992). Toxicology, structure-function relationship, and human and environmental health impacts of polychlorinated biphenyls: progress and problems. Environ. Health Perspect. 100: 259-268.

Sambrook, J., E. F. Fritsch and T. Maniatis (1989). Molecular Cloning: a Laboratory Manual, second edition. Cold Spring Harbor, Cold Spring Harbor Laboratory Press.

Sanborn, J. R., W. F. Childers and R. L. Metcalf (1975). Uptake of three polychlorinated biphenyls, DDT, and DDE by the green sunfish, Lepomis cyanellus Raf. Bull. Environ. Contam. Toxicol. 13: 209-217.

Sassa, S., O. Sugita, N. Ohnuma, S. Imajo, T. Okumura, T. Noguchi and A. Kappas (1986). Studies of the influence of chloro-substituent sites and conformational energy in polychlorinated biphenyls on uroporphyrin formation in chick-embryo liver cell cultures. Biochem. J. 235: 291-296.

Savas, U., K. K. Bhattacharyya, M. Christou, D. L. Alexander and C. R. Jefcoate (1994). Mouse cytochrome P-450EF, representative of a new 1B subfamily of cytochrome P-450s. Cloning, sequence determination, and tissue expression. J. Biol. Chem. 269: 14905-11.

Sawyer, T. and S. Safe (1982). PCB isomers and congeners: induction of aryl hydrocarbon hydroxylase and ethoxyresorufin O-deethylase enzyme activities in rat hepatoma cells. Toxicol. Lett. 13: 87-94.

Schantz, M. M., B. J. Koster, S. A. Wise and P. R. Becker (1993). Determination of PCBs and chlorinated hydrocarbons in marine mammal tissues. Sci. Total Environ. 139/140: 323-345.

Schnellman, R. G., C. W. Putnam and I. G. Sipes (1983). Metabolism of 2,2',3,3',6,6'hexachlorobiphenyl and 2,2',4,4',5,5'-hexachlorobiphenyl by human hepatic microsomes. Biochem. Pharmacol. 32: 3233-3239.

Schwartz, L. (1936). Dermatitis from synthetic resins and waxes. Amer. J. Public Health 26: $586-592$.

Schwartz, L. (1943). An outbreak of halowax acne ("cable rash") among electricians. Jour. A. M. A. 122: 158-161.

Shain, W., B. Bush and R. Seegal (1991). Neurotoxicity of polychlorinated biphenyls: structure-activity relationship of individual congeners. Toxicol. Appl. Pharmacol. 111: $33-42$.

Sherwood, M. J. and A. J. Mearns (1977). Environmental significance of fin erosion in Southern California demersal fishes. Ann. N.Y. Acad. Sci. 298: 177-189. 
Shimada, T., Y. Imai and R. Sato (1981). Covalent binding of polychlorinated biphenyls to proteins by reconstituted monooxygenase system containing cytochrome $P-450$. Chem.Biol. Interact. 38: 29-44.

Shimada, T. and R. Sato (1978). Covalent binding in vitro of polychlorinated biphenyls to microsomal macromolecules. Biochem. Pharmacol. 27: 585-593.

Shimada, T. and R. Sato (1980). Covalent binding of polychlorinated biphenyls to rat liver microsomes in vitro: nature of reactive metabolites and target macromolecules. Toxicol. Appl. Pharmacol. 55: 490-500.

Shimada, T. and Y. Sawabe (1983). Activation of 3,4,3',4'-tetrachlorobiphenyl to protein bound metabolites by the rat liver cytochrome P-448-containing monooxygenase system. Toxicol. Appl. Pharmacol. 70: 486-493.

Sinclair, P., J. Frezza, J. Sinclair, W. Bement, S. Haugen, J. Healey and H. Bonkovsky (1989). Immunochemical detection of different isoenzymes of cytochrome P-450 induced in chick hepatocyte cultures. Biochem. J. 258: 237-245.

Sinclair, P. R., W. J. Bement, H. L. Bonkovsky, R. W. Lambrecht, J. E. Frezza, J. F. Sinclair, A. J. Urquhart and G. H. Elder (1986). Uroporphyrin accumulation produced by halogenated biphenyls in chick embryo hepatocytes: reversal of the accumulation by piperonyl butoxide. Biochem. J. 237: 63-71.

Sinclair, P. R., W. J. Bement, H. L. Bonkovsky and J. F. Sinclair (1984). Inhibition of uroporphyrinogen decarboxylase by halogenated biphenyls in chick hepatocyte cultures. Essential role for induction of cytochrome P-448. Biochem. J. 222: 737-748.

Sindermann, C. J. (1979). Pollution-associated diseases and abnormalities of fish and shellfish: a review. Fish. Bull. 76: 717-749.

Sivarajah, K., C. S. Franklin and W. P. Williams (1978). Some histopathological effects of Aroclor 1254 on the liver and gonads of rainbow trout, Salmo gairdneri and carp, Cyprinus carpio. J. Fish. Biol. 13: 411-414.

Smith, P. K., R. I. Krohn, G. T. Hermanson, A. K. Mallia, F. H. Gartner, M. D. Provenzano, E. K. Fujimoto, N. M. Goeke, B. J. Olson and D. C. Klenk (1985). Measurement of protein using bicinchoninic acid. Anal. Biochem. 150: 76-85.

Smolowitz, R. M., M. E. Hahn and J. J. Stegeman (1991). Immunohistochemical localization of cytochrome P450IA1 induced by 3,3',4,4'-tetrachlorobiphenyl and by 2,3,7,8-tetrachlorodibenzofuran in liver and extrahepatic tissues of the teleost Stenotomus chrysops (scup). Drug Metab. Dispos. 19: 113-123.

Smolowitz, R. M., M. E. Schultz and J. J. Stegeman (1992). Cytochrome P4501A induction in tissues, including olfactory epithelium, of topminnows (Poeciliopsis spp.) by waterborne benzo(a)pyrene. Carcinogenesis 13: 2395-2402.

Snowberger, E. A. and J. J. Stegeman (1987). Patterns and regulation of estradiol metabolism by hepatic microsomes from two species of marine teleosts. Gen. Comp. Endocrinol. 66: 256-265. 
Song, B. J., H. V. Gelboin, S. S. Park, C. S. Yang and F. J. Gonzalez (1986).

Complementary DNA and protein sequences of ethanol-inducible rat and human cytochrome P450s. Transcriptional and post-transcriptional regulation of the rat enzyme. J. Biol. Chem. 261: 16689-16697.

Spitsbergen, J. M., J. M. Kleeman and R. E. Peterson (1988a). 2,3,7,8-

Tetrachlorodibenzo- $p$-dioxin toxicity in yellow perch (Perca flavescens). J. Toxicol. Environ. Health 23: 359-383.

Spitsbergen, J. M., J. M. Kleeman and R. E. Peterson (1988b). Morphologic lesions and acute toxicity in rainbow trout (Salmo gairdneri) treated with 2,3,7,8-tetrachlorodibenzo-pdioxin. J. Toxicol. Environ. Health 23: 333-358.

Spitsbergen, J. M., J. M. Kleeman, K. A. Schat and R. E. Peterson (1988c). Interaction of 2,3,7,8-tetrachlorodibenzo-p-dioxin (TCDD) with the resistance of rainbow trout, Salmo gairdneri, to infectious hematopoietic necrosis virus. J. Fish. Dis. 11: 73-83.

Spitsbergen, J. M., M. K. Walker, J. R. Olson and R. E. Peterson (1991). Pathologic alterations in early life stages of lake trout, Salvelinus namaycush, exposed to 2,3,7,8tetrachlorodibenzo-p-dioxin as fertilized eggs. Aquat. Toxicol. 19: 41-72.

Stadnicki, S. S., F. S. D. Lin and J. R. Allen (1979). DNA single strand breaks caused by 2,2',5,5'-tetrachlorobiphenyl and its metabolites. Res. Commun. Chem. Pathol.

Pharmacol. 24: 313-327.

Staudt, H., F. Lichtenberger and V. Ullrich (1974). The role of NADH in uncoupled microsomal monooxygenations. Eur. J. Biochem. 46: 99-106.

Stegeman, J. J. (1989). Cytochrome P450 forms in fish: catalytic, immunological and sequence similarities. Xenobiotica 19: 1093-1110.

Stegeman, J. J. and R. L. Binder (1979). High benzo[a]pyrene hydroxylase activity in the marine fish Stenotomus versicolor. Biochem. Pharmacol. 28: 1686-1688.

Stegeman, J. J., R. L. Binder and A. Orren (1979). Hepatic and extrahepatic microsomal electron transport components and mixed-function oxygenases in the marine fish Stenotomus versicolor. Biochem. Pharmacol. 28: 3431-3439.

Stegeman, J. J. and M. E. Hahn (1994). Biochemistry and molecular biology of monooxygenases: Current perspectives on the forms, function and regulation of cytochromes P450. Aquatic Toxicology: Cellular, Molecular and Biochemical Perspectives. Malins, D. and G. Ostrander (ed.). Boca Raton, CRC Press. 87-206.

Stegeman, J. J., M. E. Hahn, R. Weisbrod, B. R. Woodin, J. S. Joy, S. Najibi and R. A. Cohen (in press). Induction of cytochrome P4501A1 by Ah-receptor agonists in porcine aorta endothelial cells in culture, and CYP1A1 activity in intact cells. Mol. Pharmacol.

Stegeman, J. J., A. V. Klotz, B. R. Woodin and A. M. Pajor (1981). Induction of hepatic cytochrome P-450 in fish and the indication of environmental induction in scup. Aquat. Toxicol. 1: 197-212.

Stegeman, J. J., M. R. Miller and D. E. Hinton (1989). Cytochrome P450IA1 induction and localization in endothelium of vertebrate (teleost) heart. Mol. Pharmacol. 36: 723-729. 
Stegeman, J. J., R. M. Smolowitz and M. E. Hahn (1991). Immunohistochemical localization of environmentally induced cytochrome P450IA1 in multiple organs of the marine teleost Stenotomus chrysops (scup). Toxicol. Appl. Pharmacol. 110: 486-504.

Stegeman, J. J., B. R. Woodin, A. V. Klotz, R. E. Wolke and N. R. Orme-Johnson (1982). Cytochrome P-450 and monooxygenase activity in cardiac microsomes from the fish Stenotomus chrysops. Mol. Pharmacol. 21: 517-526.

Stegeman, J. J., B. R. Woodin, S. S. Park, P. J. Kloepper-Sams and H. V. Gelboin (1985). Microsomal cytochrome P-450 function in fish evaluated with polyclonal and monoclonal antibodies to cytochrome P-450E from scup (Stenotomus chrysops). Marine Environ. Res. 17: 83-86.

Stegeman, J. J., B. R. Woodin and R. M. Smolowitz (1990a). Structure, function and regulation of cytochrome P-450 forms in fish. Biochem. Soc. Trans. 18: 19-21.

Stegeman, J. J., B. R. Woodin and D. J. Waxman (1990b). Structural relatedness of mammalian cytochromes P450 IIB and cytochrome P450B from the marine fish scup (Stenotomus chrysops). FASEB J. 4: A739.

Stein, J. E., T. Hom and U. Varanasi (1984). Simultaneous exposure of English sole (Parophrys vetulus) to sediment-associated xenobiotics: Part 1 - Uptake and disposition of ${ }^{14} \mathrm{C}$-polychlorinated biphenyls and ${ }^{3} \mathrm{H}$-benzo[a]pyrene. Marine Environ. Res. 13: $97-$ 119.

Subramanian, A., S. Tanabe and R. Tatsukawa (1988). Use of organochlorines as chemical tracers in determining some reproductive parameters in Dalli-type Dall's porpoise Phocoenoides dalli. Marine Environ. Res. 25: 161-174.

Tanabe, S. (1985). Distribution, behaviour and fate of PCBs in the marine environment. Lecture by the member awarded the Okada prize of the Oceanographical Society of Japan for 1985. J. Oceanogr. Soc. Japan 41: 358-370.

Tanabe, S. (1988). PCB problems in the future: foresight from current knowledge. Environ. Poll. 50: 5-28.

Tanabe, S., B. G. Loganathan, A. Subramanian and R. Tatsukawa (1987).

Organochlorine residues in short-finned pilot whale. Possible use as tracers of biological parameters. Marine Poll. Bull. 18: 561-563.

Tanabe, S., S. Watanabe, H. Kan and R. Tatsukawa (1988). Capacity and mode of PCB metabolism in small cetaceans. Mar. Mamm. Sci. 4: 103-124.

Testa, B. and P. Jenner (1981). Inhibitors of cytochrome P-450s and their mechanism of action. Drug Metab. Rev. 12: 1-117.

Traber, P. G., W. Wang and L. Yu (1992). Differential regulation of cytochrome P-450 genes along rat intestinal crypt-villus axis. Amer. J. Physiol. 263: G215-223.

Ullrich, V. and H. Diehl (1971). Uncoupling of monooxygenation and electron transport by fluorocarbons in liver microsomes. Eur. J. Biochem. 20: 509-512. 
van der Weiden, M. E. J., J. van der Kolk, R. Bleumink, W. Seinen and M. van den Berg (1992). Concurrence of P4501A1 induction and toxic effects after administration of a low dose of 2,3,7,8-tetrachlorodibenzo-p-dioxin (TCDD) in the rainbow trout (Onchorhynchus mykiss). Aquat. Toxicol. 24: 123-142.

Van Veld, P. A., J. J. Stegeman, B. R. Woodin, J. S. Patton and R. F. Lee (1988). Induction of monooxygenase activity in the intestine of spot (Leiostomus xanthurus), a marine teleost, by dietary polycyclic aromatic hydrocarbons. Drug Metab. Dispos. 16: 659-665.

Varanasi, U., J. E. Stein, M. Nishimoto, W. L. Reichert and T. K. Collier (1987). Chemical carcinogenesis in feral fish: Uptake, activation, and detoxification of organic xenobiotics. Environ. Health Perspect. 71: 155-170.

Voorman, R. and S. D. Aust (1987). Specific binding of polyhalogenated aromatic hydrocarbon inducers of cytochrome P-450d to the cytochrome and inhibition of its estradiol 2-hydroxylase activity. Toxicol. Appl. Pharmacol. 90: 69-78.

Voorman, R. and S. D. Aust (1988). Inducers of cytochrome P-450d: influence on microsomal catalytic activities and differential regulation by enzyme stabilization. Arch. Biochem. Biophys. 262: 76-84.

Watanabe, S., T. Shimada, S. Nakamura, N. Nishiyama, N. Yamashita, S. Tanabe and R. Tatsukawa (1989). Specific profile of liver microsomal cytochrome P-450 in dolphin and whales. Marine Environ. Res. 27: 51-65.

Waterman, M. R., M. E. John and E. R. Simpson (1986). Regulation of synthesis and activity of cytochrome P-450 enzymes in physiological pathways. Cytochrome P-450 Structure, Mechanism, and Biochemistry. Ortiz de Montellano, P. R. (ed.). New York, Plenum Press. 345-386.

Wellings, S. R., C. E. Alpers, B. B. McCain and B. S. Miller (1976). Fin erosion disease of starry flounder (Platichthys stellatus) and English sole (Parophrys vetulus) in the estuary of the Duwamish River, Seattle, Washington. J. Fish. Res. Board Can. 33: 2577-2586.

White, R. D., M. E. Hahn, W. L. Lockhart and J. J. Stegeman (1994). The hepatic microsomal cytochrome P450 system in beluga whale (Delphinapterus leucas). Toxicol. Appl. Pharmacol. 126: 45-57.

Wirgin, I., G. L. Kreamer, C. Grunwald, K. Squibb, S. J. Garte and S. Courtenay (1992). Effects of prior exposure history on cytochrome P4501A mRNA induction by PCB congener 77 in Atlantic tomcod. Marine Environ. Res. 34: 103-108.

Yoshimura, H., Y. Yonemoto, H. Yamada, N. Koga, K. Oguri and S. Saeki (1987). Metabolism in vivo of 3,4,3',4'-tetrachlorobiphenyl and toxicological assessment of the metabolites in rats. Xenobiotica 17: 897-910. 